GA-A15612

$$
R-1260
$$

UC-77

PRE- AND POSTIRRADIATION EVALUATION OF TRISO Th02 PARTICLES IRRADIATED IN CAPSULE HT-34

\author{
by \\ C. A. YOUNG and C. S. JONES
}

Prepared under

Contract DE-AT03-76ET35300

for the San Francisco Operations Office

Department of Energy

DATE PUBLISHED: OCTOBER 1980 


\section{DISCLAIMER}

This report was prepared as an account of work sponsored by an agency of the United States Government. Neither the United States Government nor any agency Thereof, nor any of their employees, makes any warranty, express or implied, or assumes any legal liability or responsibility for the accuracy, completeness, or usefulness of any information, apparatus, product, or process disclosed, or represents that its use would not infringe privately owned rights. Reference herein to any specific commercial product, process, or service by trade name, trademark, manufacturer, or otherwise does not necessarily constitute or imply its endorsement, recommendation, or favoring by the United States Government or any agency thereof. The views and opinions of authors expressed herein do not necessarily state or reflect those of the United States Government or any agency thereof. 


\section{DISCLAIMER}

Portions of this document may be illegible in electronic image products. Images are produced from the best available original document. 
This report was prepared as an account of work sponsored by an agency of the United States Government. Neither the United States Government nor any agency thereof, nor any of their employees, makes any warranty, express or implied, or assumes any legal liability or responsibility for the accuracy, completeness, or usefulness of any information, apparatus, product, or process disclosed, or represents that its use would not infringe privately owned rights. Reference herein to any specific commercial product, process, or service by trade name, trademark, manufacturer, or otherwise, does not necessarily constitute or imply its endorsement, recommendation, or favoring by the United States Government or any agency thereof. The views and opinions of authors expressed herein do not necessarily state or reflect those of the United States Government or any agency thereof.

\section{Printed in the United States of America} Available from

National Technical Information Service

U.S. Department of Commerce

5285 Port Royal Road

Springfield, VA 22161

NTIS Price Codes: Printed Copy A07; Microfiche A01 
GA-A15612

UC-77

\title{
PRE- AND POSTIRRADIATION EVALUATION OF TRISO ThO2 PARTICLES IRRADIATED IN CAPSULE HT-34
}

\author{
by \\ C. A. YOUNG and C. S. JONES* \\ This book was prepared as an account of work sponsored by an ogenev of tha United States Governmen \\ Neither the United States Government nor any ogency thereot. nor ony of their employees, makes any \\ warranty.. express or implied, or assumes eny legal liability or responsibility lor the accuracy. \\ completeness, ar usetulness of any ino privativ owned rights. Reference herein 10 any specitic \\ represens \\ com nectorily constitute or imply its endorsement, recommendation, of favoring by the United \\ States Government or any agency theseol. The views and opinions of outhors expressed herein do not

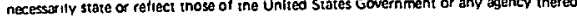 \\ Prepared under \\ Contract DE-AT03-76ET35300 \\ for the San Francisco Operations Office \\ Department of Energy
}

*Present address: Solar Research Institute, Golden, Colorado DATE PUBLISHED: OCTOBER 1980

\section{GENERAL ATOMIC COMPANY}


THIS PAGE

\section{WAS INTENTIONALLY \\ LEFT BLANK}


Capsule HT-34 was irradiated jointly by General Atomic Company (GA) and Oak Ridge National Laboratory (ORNL). This report presents the pre- and postirradiation evaluation conducted by GA. The purpose of the test was to characterize the mechanical and chemical performance and fission product release of TRISO $\mathrm{ThO}_{2}$ particles. Sixteen TRISO $\mathrm{ThO}_{2}$ samples, which had been fabricated in the production-line (240-mm-i.d.) coater, were irradiated at approximately $1200^{\circ} \mathrm{C}$ and $1450^{\circ} \mathrm{C}$ to neutron fluences of 5.1 to $10.2 \times 10^{25}$ $\mathrm{n} / \mathrm{m}^{2}$ (E > $\left.29 \mathrm{fJ}\right)_{\mathrm{HTGR}}$, and burnups of 5.1 to $12.7 \%$ FIMA.

Following are the results of the postirradiation examination:

1. The OPyC coating failure of the $800-\mu m$-diameter particles irradiated at $1200^{\circ} \mathrm{C}$ was $\leq 1.8 \%$.

2. The pressure-vessel model overpredicted failure up to seven times the observed failure for the samples irradiated at $1200^{\circ} \mathrm{C}$.

3. Palladium attack and internal corrosion of the SiC coating was observed in the samples irradiated at $1200^{\circ} \mathrm{C}$.

4. Internal corrosion of the SiC coating caused SiC failure up to $100 \%$ in the samples irradiated at $1450^{\circ} \mathrm{C}$.

5. An average of $16 \%$ and $90 \%$ of the Cs was released from failed particles irradiated at $1200^{\circ} \mathrm{C}$ and $1450^{\circ} \mathrm{C}$, respectively, after $2686 \mathrm{~h}$ of irradiation. 
THIS PAGE

\section{WAS INTENTIONALLY LEFT BLANK}


CONTEN่TS

ABSTRACT . . . . . . . . . . . . . . . . . . . . . . . $i i i$

1. INTRODUCTION . . . . . . . . . . . . . . . . . . . 1-1

1.1. Scope . . . . . . . . . . . . . . . . . . . . 1-1

1. .. Ohjertives . . . . . . . . . . . . . . . . . . 1-1

2. CAPSUlE DESIGN . . . . . . . . . . . . . . . . . . 2-1

2.1. Description . . . . . . . . . . . . . . 2-1

2.2. Irradiation Conditions . . . . . . . . . . . . . 2-1

3. UNIRRADIATED COATED PARTICLE BATCHES . . . . . . . . . . . . . . 3-1

3.1. Description . . . . . . . . . . . . . . . . 3-1

3.1.1. Parent Batches . . . . . . . . . . . . . 3-1

3.1.2: Capsule Samples . . . . . . . . . . . . 3-3

3.1.3. End-plug Samples............... 3-4

3.2. Fabrication of the Parent Batches . . . . . . . . . : 3-5

3.3. Preparation of Capsule Samples . . . . . . . . . . 3-6

3.4. Properties . . . . . . . . . . . . . . . . . 3-9

3.4.1. Parent Batches ............... . 3-9

3.4.2. Capsule Specimen. . . . . . . . . . . . 3-11

4. CATGUle pARAMETERS . . . . . . . . . . . . . . . . . . 4-1

4.1. Operating History .. . . . . . . . . . . . . 4-1

4.2. Fluence and Burnup Analysis .............. . 4-1

4.3. Thermal Analysis .. . . . . . . . . . . . . 4-1

5. RESULTS OF POSTIRRADIATION EXAMTNATIONS . . . . . . . . . . 5-I

5.1. Visual Examination . . . . . . . . . . . . . . 5-1

5.2. Metallographic Examjnation. . . . . . . . . . . 5-2

5.3. Gamma-Ray Spectrometry Analysis . . . . . . . . . . 5-4

5.4. Fission Gas Release .. . . . . . . . . . . . . 5-5

5.5. Electron Microprobe Examination . . . . . . . . . . 5-7

6. ntSCUSSION . . . . . . . . . . . . . . . . 6-1

6.1. Fission Product Release . . . . . . . . . . . 6-1 
6.2. Chemical Performance ............... . . 6-6

6.3. Mechanical Performance . . . . . . . . . . . . 6-9

7. SUMMARY AND CONCLUSIONS ................... . . . . . 7-1

8. ACKNOWLEDGMENTS . . . . . . . . . . . . . . . . . 8-1

9. REFERENCES . . . . . . . . . . . . . . . . . . . . . 9-1

\section{FIGURES}

2-1. Schematic drawing of a typical HT capsule . . . . . . . . 2-3

3-1. Typical internal flaws in the SiC coating of a TRISO-ThO ${ }_{2} \cdot$. particle ................... . . 3-12

3-2. Design test matrix of TRISO $\mathrm{ThO}_{2}$ particles for capsule HT-34. Particles made in $240-$ mm-diam coater using $\mathrm{H}_{2}$ dilution for the OPyC deposition .......... . . . . . . 3-13

3-3. Selection and characterization procedure for HT-34-capsule specimens . . . . . . . . . . . . . . . . . . . . .

3-4. Influence of various particle components on total density (one component varied at a time; other components kept at nominal value) ................. 3-15

3-5. Comparison of diverse particle types in low density fraction from particle batch 6252-14-0200 ......... 3-16

3-6. Representative photomicrographs of TRISO-coated $\mathrm{ThO}_{2}$ hatch 6252-07-020 for capsule HT-34: stereo view and Xradiograph .................... 3-17

3-7. Representative photomicrographs of TRISO coated $\mathrm{ThO}_{2}$ balch 6252-07-020 for capsule HT-34: bright field and polarized light . . . . . . . . . . . . . . . . 3-18

3-8. Representative photomicrographs of TRISO-coated Th0 2 batch 6252-13-010 for capsule HT-34: stereo view and Xradiograph .. . . . . . . . . . . . . . 3-19

3-9. Representative photomicrographs of TRISO-coated ThO 2 batch 6252-13-010 for capsule HT-34: bright field and polarized light...................... . 3-20

3-10. Representative photomicrographs of TRISO-coated $\mathrm{ThO}_{2}$ batch 6252-14-020 for capsule $\mathrm{HI}^{\mathrm{I}}-34$ : stereo view and $\mathrm{X}-$. radiograph . . . . . . . . . . . . . . . . . . . . . 3-21

3-11. Representative photomicrographs of TRISO-coated $\mathrm{ThO}_{2}$ batch 6252-14-020 for capsule HT-34: bright field and polarized light . . . . . . . . . . . . . . . . . 3-22

3-12. Representative photomicrographs of TRISO-coated $\mathrm{ThO}_{2}$ batch 6252-14-020 for capsule HT-34: 'stereo view and Xradiograph 
FIGURES (Continued)

3-13. Representative photomicrographs of TRISO-coated $\mathrm{ThO}_{2}$ batch 6252-14-020 for capsule HT-34: bright field and polarized light .. . . . . . .. . . . . . . . . . 3-24

3-14. Representative photomicrographs of TRISO-coated $\mathrm{ThO}_{2}$ batch 6252-15-020 for capsule HT-34: stereo view and Xradiograph . . . . . . . . . . . . . . . . 3-25

3-15. Representative photomicrographs of TRISO-coated $\mathrm{ThO}_{2}$ batch 6252-15-020 for capsule HT-34: bright field and polarized light ..................... 3-26

3-16. Representative photomicrographs of TRISO-coated $\mathrm{ThO}_{2}$ batch 6252-16-020 for capsule HT-34: stereo view and Xradiograph . . . . . . . . . . . . . . . . . 3-27

3-17. Representative photomicrographs of TRISO-coated $\mathrm{ThO}_{2}$ batch 6252-16-010 for capsule HT-34: bright field and polarized light . . . . . . . . . . . . . . . . . . . 3-28

3-18. Representative photomicrographs of TRISO-coated $\mathrm{ThO}_{2}$ batch 6252-17-010 for capsule HT-34: stereo view and Xradiograph . . . . . . . . . . . . . . . 3-29

3-19. Representative photomicrographs of TRISO-coated $\mathrm{ThO}_{2}$ batch 6252-17-010 for capsule HT-34: bright field and polarized light . . . . . . . . . . . . . . . . . 3-30

3-20. Representative photomicrographs of TRISO-coated $\mathrm{ThO}_{2}$ batch 6252-20-010 for capsule HT-34: stereo view and Xradiograph . . . . . . . . . . . . . . . . . . 3-31

3-21. Representative photomicrographs of TRISO-coated $\mathrm{ThO}_{2}$ batch 6252-20-010 for capsule HT-34: bright field and polarized light...................... 3-32

4-1. Results of ORNL thermal analysis ............. 4-3

5-1. Photomicrograph of a representative example of OPyC coating failure of a particle irradiated in the low temperature magazine; sample 10 had an average temperature of $1240^{\circ} \mathrm{C}$ and a fluence of $7.5 \times 10^{25} \mathrm{n} / \mathrm{m}^{2}(\mathrm{E}>29 \mathrm{fJ})_{\mathrm{HTGR}} . . . . .5-9$

5-2. Phulumicrographs of a typical samplc with zero pressure vessel and OPyC coating failure irradiated in the low Lemperature magazinc; aamplc 8 had an average temperature of $1220^{\circ} \mathrm{C}$ and a fluence of $7.0 \times 10^{25} \mathrm{n} / \mathrm{m}^{2}$ ( $\left.\mathrm{E}>29 \mathrm{fJ}\right)_{\mathrm{HTGR}} \cdot{ }^{\prime}$ - 10

5-3. Photomicrographs of total coating failure observed in a sample (6252-17-0161-001) irradiated at $1460^{\circ} \mathrm{C}$ to a fluence of $10.0 \times 10^{25} \mathrm{n} / \mathrm{m}^{2}(\mathrm{E}>29 \mathrm{fJ})_{\mathrm{HTGR}} \cdot . \cdot . . . \cdot 5-11$ 
FIGURES (Continued)

5-4. Photomicrographs of sample 6252-14-0161-001 . . . . . . . 5-12

5-5. Photomicrographs of typical particle with a 72- $\mu$ m-thick buffer (6252-14-0261-002) irradiated at $1210^{\circ} \mathrm{C}$ to a burn-up of $6.0 \%$ FIMA . . . . . . . . . . . . . . . 5-13

5-6. Photomicrographs of two particles (6252-07-0262-002) irridated at $1430^{\circ} \mathrm{C}$ to a burnup of $10.9 \%$ FIMA and a

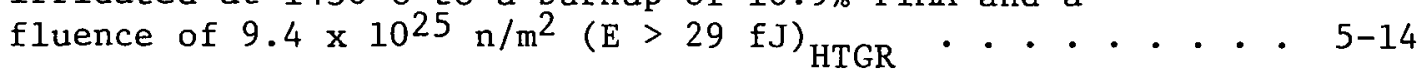

5-7. Photomicrographs of representative particles irradiated at $1240^{\circ} \mathrm{C}$ to a burn-up of $7.9 \%$ FIMA which showed SiC coatings attacked around the circumference of the coating . . . . . 5-15

5-8. Photomicrographs of two particles irradiated at $1240^{\circ} \mathrm{C}$ to a burn-up of $7.9 \%$ FIMA showing a localized attack of the SiC coating.................... 5-16

5-9. Photomicrograph of fracturing of the SiC coating near the inner surface of particles irradiated at $1180^{\circ} \mathrm{C}$ to a fluence of $8.2 \times 10^{25} \mathrm{n} / \mathrm{m}^{2}(\mathrm{E}>29 \mathrm{fJ})_{\mathrm{HTGR}} \cdot . . . . . .5-17$

5-10. Photomicrographs of kernel migration of a particle irradiated at $1490^{\circ} \mathrm{C}$ to a burn-up of $10.5 \%$ FIMA . . . . . . . 5-18

5-11a. Histograms of the measured Cs-137/2r-95 ratios. The measured/predicted $\mathrm{Cs} / \mathrm{Zr}$ ratios are also given . . . . . 5-19

5-11b. Histograms of Cs-137/Zr-95 ratios. The measured/predicted $\mathrm{Cs} / \mathrm{Zr}$ ratios are also given. The figure indicales the particles that released $\mathrm{Cs}$. . . . . . . . . . 5-20

5-11c. Histograms of $\mathrm{Cs}-137 / \mathrm{Zr}-95$ ratios. The measured/predicted $\mathrm{Cs} / \mathrm{Zr}$ ratios are alsu given. The figure indicates the particles that released $\mathrm{Cs}$. . . . . . . . . . . . 5-21

5-11d. Histograms of $\mathrm{Cs}-137 / \mathrm{Zr}-95$ ratios. The measured/predicted $\mathrm{Cs} / \mathrm{Zr}$ ratios are also given. The figure indicates the particles that released Cs . . . . . . . . . . 5-22

5-1le. Histograms of $\mathrm{Cs}-137 / \mathrm{Zr}-95$ ratios. The measured/predicted $\mathrm{Cs} / \mathrm{Zr}$ ratios are also given. The figure indicates the particles that released Cs . . . . . . . . . . . 5-23

5-11f. Histograms of $\mathrm{Cs}-137 / \mathrm{Zr}-95$ ratios. The measured/predicted $\mathrm{Cs} / \mathrm{Zr}$ ratios are also given. The figure indicates the particles that released Cs . . . . . . . . . . . 5-24

5-12. Results of electron microprobe analysis of a SiC reaction zone of a particle (6252-14-0161-001) irradiated at $1240^{\circ} \mathrm{C}$ to a burnup of $7.6 \%$ FIMA and a fluence of $7.5 \times 10^{25} \mathrm{n} / \mathrm{m}^{2}$ (E > $29 \mathrm{fJ})_{\mathrm{HTGR}}$. . . . . . . . . . . . . . . 5-25 
FIGURES (Continued)

5-13a. Results of electron microprobe examination of SiC corrosion of sample 17 ; irradiated at $1430^{\circ} \mathrm{C}$ to a burnup of $10.9 \%$

FIMA . . . . . . . . . . . . . . . . 5-26

5-13b. Results of electron microprobe of center of kernel of sample 17 irradiated at $1430^{\circ} \mathrm{C}$ to a burnup of $10.9 \%$ FIMA . . . . . 5-27

6-1. Measured and predicted fission products of capsule HT-34 samples . . . . . . . . . . . . . . . . 6-12

6-2. Ratios of measured to predicted $\mathrm{C}-137 / \mathrm{Zr}-95$ ratio of $1200^{\circ} \mathrm{C}$ samples ................. 6-13

6-3. Calculated amount of Cs-137 retained in particles from each sample... . . . . . . . . . . . . . 6-14

6-4. Graph of SiC thinning versus temperature. The points given are the amount of SiC fission product attack measured on metallographic polished sections of HT-34 TRISO $\mathrm{ThO}_{2}$ particles. The solid lines are the bounds of the square-root-oftime model for SiC thinning . . . . ... . . . . 6-15

TABLES

2-1. Description and design irradiation conditions of TRISO $\mathrm{ThO}_{2}$ samples for capsule $\mathrm{HT}-34$. . . . . . . . . . . . . . 2-4

3-1. Primary design variables of TRISO $\mathrm{ThO}_{2}$ parent particle batches for capsule HT-34 . . . . . . . . . . . . . . 3-33

3-2. Variables to be tested in capsule HT-34 . . . . . . . . 3-34

3-3. Location and description of particles in end plugs of

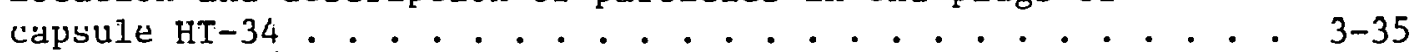

3-4. Fabrication condtions of TRISO $\mathrm{ThO}_{2}$ parent batches for capsule HT-34 . . . . . . . . . . . . . . . . 3-37

3-5. Numerical cross reference of HT-34 capsule specimens . . . 3-39

3-6. Calculation of particle loadings for capsule HT-34 . . . . . 3-40

3-1. Input parameters used in TRISO monte-noco code . . . . . . 3-41

3-8. Predicted particle performance for capsule HT-34 . . . . . . 3.442

3-9. Kernel properties of TRISO $\mathrm{ThO}_{2}$ parent batches for capsule $\mathrm{HT}-34$. . . . . . . . . . . . . . . 3-43

3-10. Coating properties of TRISO Th0 2 parent batches for capsule $\mathrm{HT}-34$.................. . . 3-45

3-11. Properties of total coatcd particles of TRISO Th0 2 parent hatches for capsule HT-34........... . . 3-47 


\section{TABLES (Continued)}

3-12. Measured properties of particle samples for capsule HT-34 . 3-49

3-13. Summary of TRISO $\mathrm{ThO}_{2}$ particle samples for capsule HT-34 • . 3-51

3-14. Comparison of HT-34 parent batches with capsule specimens . . 3-53

4-1. Reactor operating history. . . . . . . . . . . . . . 4-19

4-2. Neutron fluences and burnups of samples... . . . . . . 4-20

4-3. Time-averaged maximum surface temperatures of samples . . . 4-21

4-4. Power per particle of samples............ . 4-22

4-5. Time-averaged kernel temperatures of samples . . . . . 4-23

5-1. Results of visual examination . . . . . . . . . . . . 5-28

5-2. Results of metallographic examination . . . . . . . . . 5-29

5-3. Results of $\gamma$-coating of HT-34 samples........... . 5-31

5-4. Fission product ratios . . . . . . . . . . . . . 5-33

5-5. Triga fission gas release data and calculated particle failure................. . . 5-34

6-1. Summary of coated particle failure of TRISO $\mathrm{ThO}_{2}$ samples . * 6-16

6-2. Comparison of predicted and measured Cs retained in failed TRISO $\mathrm{ThO}_{2}$ particles in capsule HT-34 . . . . . . . . . 6-17

6-3. Comparison of SiC attack and Pd per particle of TRISO $\mathrm{ThO}_{2}$
particles......................... 6-18 


\section{INTRODUCTION}

\subsection{SCOPE}

\section{1}

The irradiation of capsule HT-34 was part of a continuing cooperative effort. between GA and ORNL, funded by the Department of Energy-sponsored High-Temperature Gas-Cooled Reactor (HTGR) Fuels and Core Development Program. The irradiation performance of eight TRISO-coated $\mathrm{ThO}_{2}$ particle batches, fabricated in the 240-mm-i.d. HTGR pilot-plant coater, were characterized in this test. Sixteen samples were irradiated in the uninstrumented capsule in the target facility of the High-Flux Isotope Reactor (HFIR) at ORNL at temperatures of approximately $1200^{\circ}$ and $1450^{\circ} \mathrm{C}$, over a wide range of fluences and burnups. The HT-capsule experiments provide a rapid means of evaluating and screening fuel materials, since the high-neutron flux in the reactor allows the accumulation of high, fast neutron doses and high thorium burnups in a short time.

The fuel from capsule HT-34 was characterized after irradiation. The postirradiation examinations included a visual examination, metallographic examination, fission gas release (FGR) measurements, and fission product release measurements. The chemical, mechanical, and fission product release performances of the TRISO $\mathrm{ThO}_{2}$ particles were evaluated.

\subsection{OBJECTIVES}

The design objectives for testing TRISO-coated $\mathrm{ThO}_{2}$ particles in capsule HT-34 follow:

1. Test TRISO-coated $\mathrm{ThO}_{2}$ particles containing 450- $\mu \mathrm{m}$ kernels in an accelerated capsule. The HT-34 particles are representative of 
the candidate design for the Fort St. Vrain (FSV) HTGR, and are more retentive of fission products compared to the BISO particle.

2. Evaluate the irradiation performance of unbonded particles fabricated in the 240-mm-i.d. pilot-plant coater, using $\mathrm{H}_{2}$ for the outer pyrocarbon (OPyC) coating diluent gas; $\mathrm{H}_{2}$-diluted OPyC coatings were tested in two BISO-particle batches in capsule HT-33 (Ref. 1), but had not been previously tested in TRISO particles. One particle batch with an Ar-diluted OPyC coating was inserted in capsule HT-34 as a comparative sample.

3. Vary the design of the TRISO particle to obtain a range of pressure-vessel failure at $1200^{\circ}$ and $1450^{\circ} \mathrm{C}$; for correlation with particle stress models.

4. Evaluate the chemical behavior of TRTSO $\mathrm{ThO}_{2}$ particles irradiated at $1200^{\circ}$ and $1450^{\circ} \mathrm{C}$.

5. Test a range of density, microporosity, coating rate, and anisotropy of the OPYC coating to add to the data base in support of current specifications.

6. Evaluate Sic-coating irradiation performance as a function of the frequency of internal flaws in the SiC coating. 


\section{CAPSULE DESIGN}

\subsection{DESCRIPTION}

Capsule HT-34 contained four tapered POCO-graphite magazines housed in a primary aluminum container. [See Fig. 2-1* (Ref. 2) for a schematic drawing of a typical HT capsule.] Each magazine held 13 POCO-graphite cylindrical crucibles, which contained unbonded, coated fuel particles. Eight of the 13 crucibles housed fertile particle samples; the other five contained ORNL low-enriched uranium particles to provide an initial fission heat source and to flatten the axial temperature distribution. The magazine-end plugs housed both inert and fertile particles. GA fertile particles occupied the top two magazines in the capsule. After the four magazines were loaded into the primary container, the capsule was sealed with $99.995 \%$ pure Ar at a pressure of $0.15 \mathrm{MPa}$ ( $5 \mathrm{psig}$ ). This meant that no in-pile fission gas release could be measured. Capsule temperatures were not monitored during the irradiation, since no thermocouples were in the capsule.

Capsule HT-34 was inserted into the target facility of the HFIR in July 1977 for irradiation for five cycles. It was discharged in late November 1977 .

\subsection{IRRADIATION CONDITIONS}

The design irradiation conditions for the GA samples are given in Table 2-1. The surface temperatures of the graphite crucibles of the two magazines were designed to be $900^{\circ}$ and $1250^{\circ} \mathrm{C}$. These graphite temperatures correspond to estimated particle surface temperatures of $1200^{\circ}$ and $1450^{\circ} \mathrm{C}$, respectively. The lower temperature magazine samples reached fluences

\footnotetext{
${ }^{*}$ Figures and tables appear at the end of each section.
} 
of approximately 5.3 to $8.3 \times 10^{25} \mathrm{n} / \mathrm{m}^{2}$ (E > $29 \mathrm{fJ}$ ) $\mathrm{HTGR}$ and burnups of $5.6 \%$

to $9.1 \%$ FIMA. The fast fluences and burnups for the high temperature magazine were 9.4 to $10.4 \times 10^{25} \mathrm{n} / \mathrm{m}^{2}$ (E $\left.>29 \mathrm{fJ}\right)_{\mathrm{HTGR}}$ and $11.0 \%$ to $12.8 \% \mathrm{FIMA}$. 


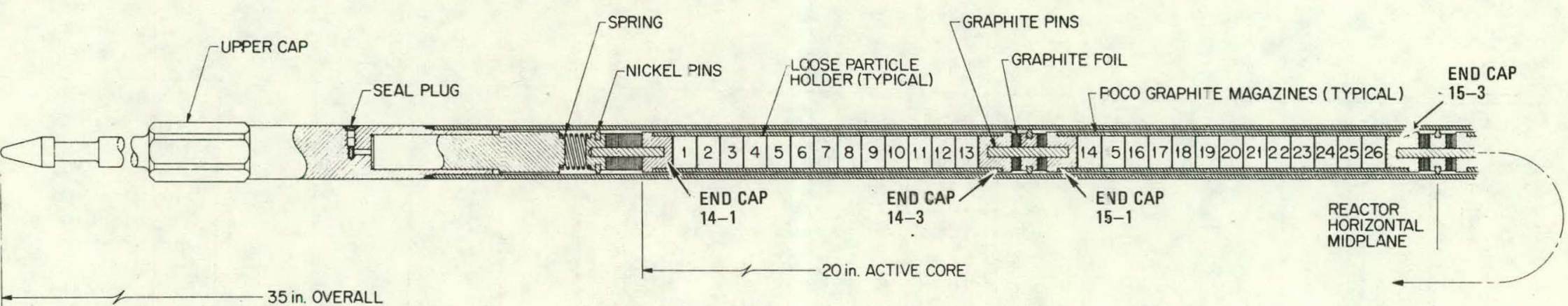

$N$
1
$\omega$

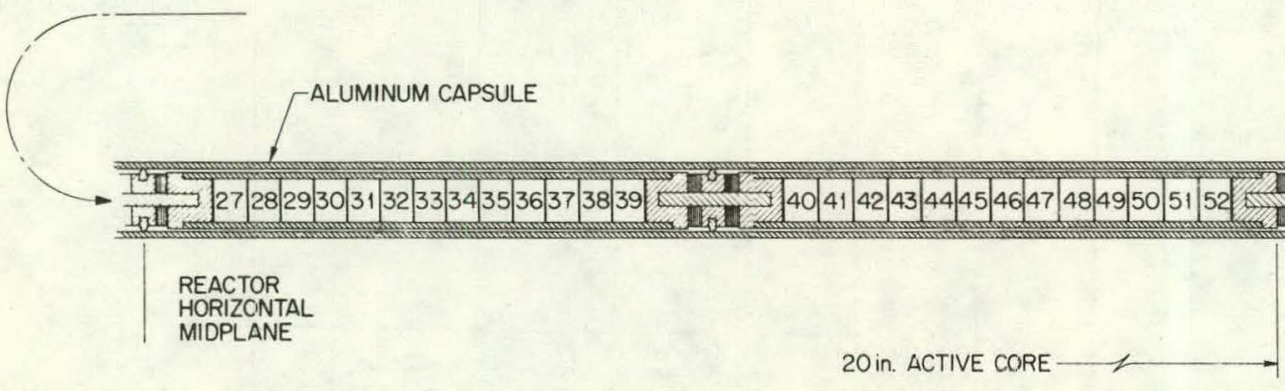

20 in. ACTIVE CORE
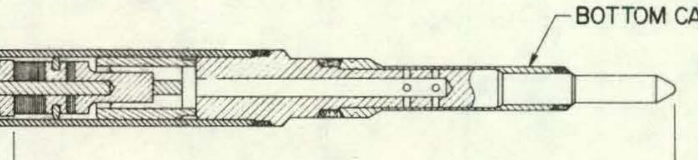

35 in. OVERALL

Fig. 2-1. Schematic drawing of a typical HT capsule 
TABLE 2-1

DESCRIPTION AND DESIGN IRRADIATION CONDITIONS OF TRISO ThO ${ }_{2}$ SAMPLES FOR CAPSULE HT- 34

\begin{tabular}{|c|c|c|c|c|c|c|c|c|c|c|c|c|c|}
\hline \multirow[b]{4}{*}{ Sample Batch No. } & \multicolumn{6}{|c|}{ Variables to be Tested } & \multirow{4}{*}{$\begin{array}{l}\text { Density-Separated } \\
\text { Fraction from } \\
\text { Farent Batch } \\
\end{array}$} & \multirow{3}{*}{\multicolumn{2}{|c|}{$\begin{array}{c}\text { Design Particle } \\
\text { Failure after } \\
\text { Irradiation }(a) \\
\end{array}$}} & \multirow[b]{4}{*}{$\begin{array}{c}\text { No. } \\
\text { Particles } \\
\end{array}$} & \multirow{2}{*}{\multicolumn{3}{|c|}{ Design Irradiation Condition }} \\
\hline & \multirow{3}{*}{$\begin{array}{l}\text { Buffer } \\
\text { Th:ckness } \\
(\mu \mathrm{m}) \\
\end{array}$} & \multirow{3}{*}{$\begin{array}{c}\text { Sic } \\
\text { Flaw } \\
\text { Frequency } \\
(\%)\end{array}$} & \multicolumn{4}{|c|}{. $\mathrm{PyC}$} & & & & & & & \\
\hline & & & $\begin{array}{l}\text { Liquid } \\
\text { Gradient }\end{array}$ & Micro- & Coating & & & & & & \multirow[b]{2}{*}{$\begin{array}{r}\text { Capsule } \\
\text { Position } \\
\end{array}$} & \multirow{2}{*}{\begin{tabular}{|c|c} 
Fast Fluence \\
{$\left[10^{25} \mathrm{n} / \mathrm{m}^{2}\right.$} \\
$(\mathrm{E} \quad 29 \mathrm{fJ})_{\mathrm{HTCR}}$ \\
\end{tabular}} & \multirow[b]{2}{*}{$\begin{array}{c}\text { Burnup } \\
\text { (\% FIMA) }\end{array}$} \\
\hline & & & $\begin{array}{c}\text { Density } \\
\left(\mathrm{Mg} / \mathrm{m}^{3}\right) \\
\end{array}$ & $\begin{array}{r}\text { porosity } \\
(\mathrm{m} \ell / \mathrm{kg})\end{array}$ & $\begin{array}{c}\text { Rase } \\
(\mu \mathrm{m} / \mathrm{min})\end{array}$ & $\begin{array}{c}\text { Diluent } \\
\text { Gas }\end{array}$ & & OPyC & $\begin{array}{c}\text { Pressure } \\
\text { Vesse1 }\end{array}$ & & & & \\
\hline \multicolumn{14}{|l|}{ Low temp magazine (b) } \\
\hline 6252-20-0161-001 & 83 & 6 & 1.98 & 59 & 8.4 & $\mathrm{H}_{2}$ & Nominal & Low & 0 & 56 & 2 & 5.3 & 5.6 \\
\hline $6252-07-0262-001(c)$ & 60 & 38 & 1.80 & 48 & 5.0 & $\mathrm{Ar}$ & Nominal & Low & 0 & 57 & 4 & 5.9 & 6.2 \\
\hline $6252-14-0261-001$ & 27 & 29 & 1.97 & 54 & 7.1 & $\mathrm{H}_{2}$ & High & Low & Moderate & 56 & 5 & 6.2 & 6.5 \\
\hline $6252-14-0171-001$ & 35 & 9 & 1.97 & 57 & 7.1 & $\mathrm{H}_{2}$ & High & Low & Moderate & 56 & 7 & 6.8 & 7.1 \\
\hline $6252-13-0161-001$ & 57 & 16 & 1.79 & 57 & 8.3 & $\mathrm{H}_{2}$ & Nominal & Low & 0 & 57 & 8 & 7.1 & 7.5 \\
\hline $6252-14-0161-001$ & 63 & 巳 & 1.97 & 57 & 7.6 & $\mathrm{H}_{2}$ & Nominal & Low & 0 & 57 & 10 & 7.6 & 8.1 \\
\hline $6252-15-0161-001$ & 56 & 12 & 1.81 & 21 & 5.0 & $\mathrm{H}_{2}$ & Noninal & Low & 0 & 56 & 11 & 7.9 & 8.5 \\
\hline $6252-16-0161-001$ & 57 & 12 & 1.96 & 25 & 5.3 & $\mathrm{H}_{2}$ & Nominal & Low & 0 & 56 & 13 & 8.3 & 9.1 \\
\hline \multicolumn{14}{|l|}{ High temp magazine ${ }^{(d)}$} \\
\hline $6252-20-0161-002$ & 82 & 6 & 1.98 & 59 & 8.0 & $\mathrm{H}_{2}$ & Nominal & Moderate & Moderate & 80 & 15 & 9.4 & 11.0 \\
\hline $6252-07-0262-002(c)$ & 57 & 38 & 1.80 & 48 & 5.6 & $\mathrm{Ar}$ & Nominal & Moderate & High & 81 & 17 & 9.8 & 11.5 \\
\hline $6252-14-0161-002$ & 62 & 9 & 1.97 & 57 & 7.6 & $\mathrm{H}_{2}$ & Nominal & Moderate & High & 81 & 18 & 9.9 & 11.7 \\
\hline $6252-14-0271-001$ & 86 & 29 & 1.97 & 54 & 8.0 & $\mathrm{H}_{2}$ & Low & Moderate & Moderate & 83 & 20 & 10.1 & 12.1 \\
\hline $6252-14-0181-001$ & 91 & 10 & 1.97 & 57 & 8.1 & $\mathrm{H}_{2}^{2}$ & Low & Moderate & Moderate & 82 & 21 & 10.2 & 12.2 \\
\hline $6252-17-0161-001$ & 80 & 5 & 1.95 & 28 & 5.0 & $\mathrm{H}_{2}$ & Ncminal & Moderate & Moderate & 81 & 23 & 10.4 & 12.5 \\
\hline $6252-15-0171-001$ & 84 & 12 & 1.81 & 21 & 5.6 & $\mathrm{H}_{2}$ & Lcw & Moderate & Moderate & 81 & 24 & 10.4 & 12.6 \\
\hline $6252-13-0171-001$ & 79 & 16 & 1.79 & 57 & 8.5 & $\mathrm{H}_{2}^{2}$ & Low & Moderate & Moderate & 85 & 26 & 10.4 & 12.8 \\
\hline
\end{tabular}

(a) Design failure range $=1$ ow $<5 \%$; moderate $20-30 \%$; and high $50-70 \%$

(b) Low temp magazine $-1200^{\circ} \mathrm{C} ; 0.0234 \mathrm{~g}$ Th-232/position

(c) Sample previously irradiated in capsules HT-31 and HT-33

(d) High temp magazine $-1500^{\circ} \mathrm{C} ; 0.0331 \mathrm{~g}$ Th-232/position 


\section{UNIRRADIATED COATED PARTICLE BATCHES}

\subsection{DESCRIPTION}

\subsubsection{Parent Batches}

Eight TRISO-cualed partiele batchee, containing 450-um-diam $\mathrm{ThO}_{2}$ kernels, were tested in capsule HT-34. All batches were made in the 240-mm-i.d. pilot-plant coater. Primary test variables for the parent batches (Table 3-1) included buffer thickness, SiC internal flaw content, and OPyC coating density, microporosity, coating rate, and diluent gas.

The 240-mm-i.d. coater with a cone-shaped gas distributer was selected for the HTGR pilot plant. The TRISO and $\mathrm{BISO}^{\mathrm{ThO}} 2$ particles made in this coater were first tested in capsules HT-31 and HT-33 (Ref. 1). The TRISO $\mathrm{ThO}_{2}$ particles were further tested in $\mathrm{HT}-34$. Since most of the present irradiation data are based on particles made in the 127-mm-i.d. prototype coater, the results of capsules HT-31, HT-33, and HT-34 will aid in assuring that the irradiation performances of particles mede in both the small and large coaters are similar.

The parent batches were designed with either a $60-$ or $85-\mu m$ buffer thickness to accommodate a wide range of burnups. The buffer coating provides void volume to accommodate gaseous fission products. More fission gases were generated in coated particles irradiated to high burnups, and thicker buffers were required to limit pressure-vessel failure.

Capsule HT-34 tested TRISO $\mathrm{ThO}_{2}$ samples having a range of internal flaw fractions in the SiC coating. (See Fig. 3-1 for flaw example.) Internal flaws have been observed on metallographically polished sections in the SiC coating of TRISO particles made at GA. They have been more prevalent in large-diameter particles. The results of previous irradiation capsules; 
such as F-30, P13R, and P13S (Refs. 3 and 4), indicated that TRISO particles with these flaws performed satisfactorily. However, the performance of particles might be improved by reducing the SiC internal flaw content, thereby allowing a reduction in coating thickness or an increase in the fuel operating temperature.

GA began to study SiC flaws and observed that large-coater TRISO $\mathrm{ThO}_{2}$ particles for HT-31 and HT-33 had flaws (Ref. 1). When these batches were burned back to the SiC coating, a visual examination revealed gold spots. Further work showed that each gold spot was a flaw or inclusion in the SiC coating. The fraction of particles with flaws was then determined by counting gold-spotted particles. The flaw counts for capsule HT-34 batches are reported in Table 3-1. Electron microprobe analysis revealed that the flaws were primarily carbon and void.

A short-term experiment was undertaken to reduce the flaw content of SiC coatings made in the large coater for capsule HT-34. Coater batch size was found to be the only significant parameter. As a result, several HT-34 specimens were made using half-size batches $(\sim 11 \mathrm{~kg})$ in the SiC-coating run. This reduced the frequency of flawed SiC coatings from $40 \%$ to $\sim 10 \%$. It appeared that the ratio of particle-bed height to coater diameter influenced the SiC flaw content (Ref. 5). However, the flaws were never completely eliminated in this experiment. Capsule HT-34 tested a range of flaw frequencies to examine the effect of SiC flaws on irradiation performance.

Two total particle diameters were tested in capsule HT-34, bccause of the need for two buffer coating thicknesses. The batches having. -60 rmbuffer coatings had a diameter of $\sim 800 \mu \mathrm{m}$. These batches are representative of the candidate design for TRISO-coated 450-mm $\mathrm{ThO}_{2}$ particles for the FSV HTGR reactor (Kef, 6) and the particles tested in the FSV fuel test elements FTE-1 through FTE-8 (Ref. 7). The thick-buffer batches had a diameter of $\sim 870 \mu \mathrm{m}$. Higher OPyC coating failures have been observed in large-diameter TRISO $\mathrm{ThO}_{2}$ particles (Ref. 8 ). The results of HT-34 were expected to contribute to the data base on the size effect. 
Characterizing the outer pyrocarbon coating is part of a continuing effort to ensure adequate irradiation performance of HTGR coated particles. The density, microporosity, coating rate, and anisotropy have been found to affect particle performance significantly (Refs. 4, 8, and 9). The test matrix for the OPyC coating is given in Fig. 3-2. It consisted of two coating rates ( 5 and $8 \mu \mathrm{m} / \mathrm{min}$. ) and two liquid gradient densities ( 1.80 and 1.95 $\mathrm{Mg} / \mathrm{m}^{3}$ ). Microporosity and anisotropy also varied, since they were dependent upon coating rate and density. Particles with the selected coating rates and densities were expected (from previous results) to perform adequately during irradiation at $1200^{\circ} \mathrm{C}$.

\subsubsection{Capsule Samples}

Table 2-1 gives a general description of the 16 TRISO $\mathrm{ThO}_{2}$-particle samples for capsule HT-34. The samples were selected from the parent batches and prepared for the capsule according to the experiment objectives. The test variables and the corresponding capsule samples are given in Table 3-2. The OPyC coating characteristics were emphasized primarily in the $1200^{\circ} \mathrm{C}$ magazine; however, a11 OPyC coating parameters were tested at both temperatures. The $1450^{\circ} \mathrm{C}$ experiment, since it was a severe test, mainly evaluated mechanical (pressure vessel failure) and chemical behavior. Chemical behavior included kernel migration and attack of the SiC coating. The results were to be correlated with the particle performance models.

One intent of the experiment was to produce a range of pressure-vessel failure by adjusting the buffer thickness over the range of burnups, as shown in Table 2-1. The pressure-vessel failure model was used in the design of the TRISO $\mathrm{ThO}_{2}$ particles. Low pressure-vessel failure was expected in the low-temperature samples having a $60 \mu \mathrm{m}$-thick buffer coating. Two $1200^{\circ} \mathrm{C}$ samples had $30-\mu \mathrm{m}$ buffer coatings and were designed to have failure. The high temperature samples were irradiated to very high neutron burnups. Therefore, an 85-m buffer coating was required for low failure. 
Two samples having 60-ım-thick buffer coatings were designed to have high failure at $\sim 1450^{\circ} \mathrm{C}$. Also, the different frequencies of internal flaws in the SiC coating were tested at both $1200^{\circ}$ and $1450^{\circ} \mathrm{C}$ to determine their effect on pressure-vessel failure.

The diluent gases used during OPyC coating deposition were either $\mathrm{H}_{2}$ or Ar. One test objective was to verify that the irradiation perfornances of the two OPyC coating types are comparable. The reasons for the use of different diluent gases are discussed in Section 3.2.' One parent batch (Ar. dilution), irradiated in both capsule HT-31 and capsule HT-33, was used as a standard in both magazines of HT-34. Since temperatures were not monitored during the irradiation, the standard was to provide comparative particle performance data and assist in demonstrating that temperatures of these capsules were similar.

Table 2-1 shows that the number of $\mathrm{ThO}_{2}$ particles in different positions is limited to between 56 and 85 particles. Fuel failure within these limited groups of particles is enough to establish qualitative "gross effects" between different coating attributes. A meaningful quantitative evaluation of low fuel failure fractions versus coating attributes is not possible because of the small size of the samples in each irradiation position; e.g., 330 particles have to be examined with no observed failures to have $95 \%$ confidence that the failure fraction in this group is less than 0.01 . Thus, the HT-34 capsule was designed to serve as a screening test to determine success or failure between different particle groups and identify mechanisms contributing to particle failure.

\subsubsection{End-plug Samples}

The four end plugs of the two CA magazines contained two additional. coated-particle samples. Batch 7032-149 was $\mathrm{BISO}_{\mathrm{ThO}_{2}}$ with a Si-doped OPyC coating. The developmental si-doped pyrocarbon exhibits higher strength and improved dimensional stability under irradiation (Ref. 10), when compared 


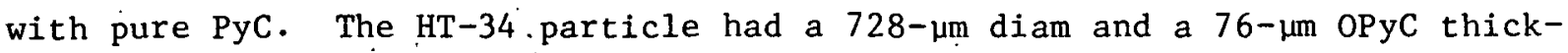
ness. The OPYC coating had a density of $2.22 \mathrm{Mg} / \mathrm{m}^{3}$ with 34 wt\% $\mathrm{Si}$. Batch 6351-04-010 was TRISO inert particles that had been burned back to the SiC coating. This batch was inserted to determine if exposed SiC coating corrodes or volatizes during irradiation. This particle had a substrate diam of $502 \mathrm{~mm}$ and a SiC density of $3.22 \mathrm{Mg} / \mathrm{m}^{3}$. The location, irradiation conditions, Th loading, and number of particles are listed in Table 3-3.

\subsection{FABRICATION OF THE PARENT BATCHES}

All of the kernels and coatings were manufactured in the HTGR pilot plant in accordance with the specifications in Ref. 11. The 450- $\mu \mathrm{m}$ kernels were made from $\mathrm{ThO}_{2}$ powder derived from a steam-denitrated Th-nitrate solution. A broth, made from the powder, was then fed into a drop column where it was spheroidized into uniform droplets and gelled into anmonia gas. The gelled spheres were dried in air at $150^{\circ} \mathrm{C}$ and sintered at $\leq 1300^{\circ} \mathrm{C}$.

The coatings were deposited on the kernels in the 240-mm-i.d. pilotplant dry coater. The fabrication conditions of each batch are given in Table 3-4. The gases entered the coating chamber through a cone-shaped gas distributor with an extension nozzle through the center of the cone. The levitating gas was dispersed at the base of the cone. The deposition and diluent gases were premixed and entered near the top of the extension nozzle.

The diluent gas has an important effect on the pyrocarbon properties. The diluent gases $\mathrm{Ar}, \mathrm{He}$, and $\mathrm{N}_{2}$ have produced acceptable pyrocarbon coatings in smaller (127 mm i.d. or less) coaters. However, pyrocarbon coatings made in the $240-m m-i . d$. coater with the cone distributor were frequently sooty and permeable when Ar was the diluent gas. Possibly, this was due to nonhomogeneous dispersion of the coating gases in the large coater bed. Hydrogen dilution reduced soot and permeability (measured by chlorine leach on BISO $\mathrm{ThO}_{2}$ particles), and produced a more uniform coating. In addition, 
the use of $\mathrm{H}_{2}$ reduced the OPyC coating thickness variability under constant coating conditions. The beneficial effects from $\mathrm{H}_{2}$ were apparently attributed to the stabilization of the decomposition of the hydrocarbon gas. Two BISO $\mathrm{ThO}_{2}$ batches in capsule $\mathrm{HT}-33$ (Ref. 1) were deposited with $\mathrm{H}_{2}$ diluent gas. Seven of the eight TRISO batches in capsule iT-34 had OPyC coatings deposited with the $\mathrm{H}_{2}$ diluent. Capsule HT-34 was expected to show whether or not this process change affects the irradiation behavior of the pyrocarbon. The OPyC coating was deposited with Ar for the other batch.

The particle batch size in the coater also affects coating properties. The buffer and inner pyrocarbon (IPyC) coatings were deposited in 15 or 17 $\mathrm{kg}$ charges. Loads of 10.5 to $22.5 \mathrm{~kg}$ were used for the SiC coating. The frequency of flaws in the SiC coating was roughly proportional to the charge size in batches with buffers approximately $60 \mu \mathrm{m}$ thick. However, the 80-mm-thick buffer batches dic not follow this trend, since they had a low frequency of flaws and were coated in large batch sizes. All opyc-coating load sizes were between 11 and $14 \mathrm{~kg}$.

\subsection{PREPARATION OF CAPSULE SAMPLES}

The preparation and characterization of the actual capsule specimens from the parent batches, described in section 3.1 and 3.2 , were conducted in accordance with the procedure shown in Fig. 3-3. "In the interest of traceability, the numerical changes in sample identification that occurred during these procedures are given in Table 3-5. The various steps in the procedure are described in detail below.

All samples for capsule HT-34 were separated by particle density into a low, mean, or high density from the parent batch. The mean-density fraction was separated out to reduce the variation in all coating attributes but primarily the buffer thickness. This reduction aided in correlating irradiation performance with coating attributes. The buffer thicknesses of the parent batches were nominally 60 or $85 \mu \mathrm{m}$. Therefore, the mean-density fractions had 60 - or $85-\mu m-t h i c k$ buffer coatings. 
The design also called for six samples taken from three of the 60- $\mu \mathrm{m}$-thick buffer parent batches to have 30- and/or 85- $\mu \mathrm{m}$ buffer thicknesses. This meant that the particles with the thinnest and thickest buffer coatings had to be separated from the parent batches. The most practical method for separating them was by total particle density. Calculations were then performed to determine what particle components (kernel and coatings) most influenced the total particle density. The density was calculated for TRISO $\mathrm{ThO}_{2}$ particles with nominal component dimensions and densities. The dimension of each component was then varied by plus and minus two standard deviations of the nominal value, while the other components were held constant. The results are given in Fig. 3-4. The slope of each line defined how much influence each component had on the total particle density. Fortunately, the buffer thickness had the most effect on the den-. sity. This fact made it easier to separate out the particles with thin and thick buffer coatings. However, the figure also shows that the high-density fraction would be expected to have larger kernels and thinner coatings. Conversely, the low-density fraction would have smaller kernels and thicker coatings.

That a particular density may be obtained through a variety of particle designs is illustrated in Fig. 3-5, which shows two different microstructures found in the same low-density fraction of batch 6252-14-0200. One consisted of a nominal OPyC and thin buffer coating, and the other of an even thinner buffer and thick OPyC coating. It is interesting to note the range of OPyC thickness and density occurring within the same batch of particles, deposited over the same period, in the same coater. The thicker OPyC coating obviously has more surface-connected porosity, as the impregnation of $\mathrm{Tl}$ indicates, and is probably of lower density.

To prepare the mean-density fractions, the mean total particle density was first measured on 100 particles from each parent batch; then the meandensity fraction was separated out from a sample of the parent batch in a density-gradient column. 
The high- and low-density fractions were separated in a beaker rather than in a density column. For example, 200 low-density particles were separated from a $30-\mathrm{g}$ particle sample using a Tl mallonate solution. All the added particles sank to the bottom. A high-density Tl solution was then added drop-by-drop to the beaker, and stirred thoroughly until approximately 200 of the least-dense particles were floating on the surface. These floating particles were skimmed off and became the low-density fraction. Conversely, the high-density fraction was obtained by preparing a solution in which all particles floated. A low-density T1 solution was then added until the required number of particles sank.

The density-separated samples were then $\mathrm{X}$-radiographed, and the dimensions were measured to determine whether they met their individual buffer-thickness requirements. For those not meeting the requirements, another density separation was made. The high-and low-density tails were radiographed in slotted brass holders, so that defective particles could be located and removed. Defective (per Ref. 11) particles were removed from a11 high- and low-density fractions, but not from the mean-density fractions, which generally had few, if any, defects.

The density separation was followed by a $1-\mathrm{h}, 1400^{\circ} \mathrm{C}$, vacuum $\left(5 \times 10^{-4}\right.$ $\mathrm{mm} \mathrm{Hg}$ ) heat treatment to remove the $\mathrm{T}$. All capsule samples and historical specimens were heated together. Following this initial heat treatment, the historical samples were removed and stored. The capsule specimens were then heat treated at $1650^{\circ} \mathrm{C}$ in Ar for $30 \mathrm{~min}$ to simulate the fuel rod heating.

After the final heat treatment, the capsule specimens were radiographed again. The number of particles needed per capsule to meet the specified Th loading (see Table 3-6) was calculated. Then, the excess particles from each capsule specimen were removed and used for OPyC and optical anisotropy $\left(\mathrm{BAF}_{\mathrm{O}}\right)$ characterization. A final radiograph was taken of the particles to be inserted in the capsule, and measurements were noted on the kernel and coating dimensions for each capsule sample. 
Stress calculation' were performed with the TRISO MONTE-NOCO computer code, using design irradiation conditions and data on selected capsule samples (see Table 3-7). Predicted failure levels for the IIT-34 samples were determined by imposing the calculated stress curves on an apparent failure stress of -3400 psii. This value was based on the stress studies of HT and P13S capsules (Ref. 12). The failure fractions, assuming both zero and $100 \%$ OPyC failure, are given in Table 9. The chance of failure was equal to the probability that the apparent failure stress was exceeded for each capsule sample. It is obvious from Table 3-8 that a meaningful test of the particle designs against the models could be realized only if the OPyC remained intact.

\subsection{PROPERTIES}

All parent batches and capsule samples were extensively characterized prior to irradiation. The results of the quality-control analysis are presented in Tables 3-9 through 3-14. Representative photomicrographs of $\mathrm{X}$-radiograph, stereo view, and metallographic. cross sections of coated particles from each parent batch are shown in Figs. 3-6 through 3-21.

\section{Coated-particle properties were measured at different stages of} preparation: On the parent batches, items measured included kernel density and impurities, coating densities, IPyC and $\mathrm{OPyC}_{\mathrm{BAF}}, \mathrm{SiC}$ flaws, faceting, accessible porosity (measured by mercury intrusion), chemical composition and impurities, defective SiC coatings, contamination, and fission gas release (FGR). The total particle density and some BAFo values were measured on the density-separated fractions. The particle dimensions, SiC and OPyC coating rates, and Th loadings were measured or calculated on the capsule-particle samples.

\subsubsection{Parent Batchès}

The properties of the parent batches are presented in Tables $3-4$ and 3-9 through 3-11. The kernel characteristics are similar for all batches. 
The $\mathrm{ThO}_{2}$ was $\geq 97.8 \%$ of theoretical density and its impurity content was low. The average diameter was very close to $450 \mu \mathrm{m}$ for all batches.

The measured properties of the coatings agreed fairly well with the design properties given in Table 3-1. Buffer thickness ranged from 54 to 63 $\mu \mathrm{m}$ and 86 to $90 \mu \mathrm{m}$. The SiC-flaw frequencles were measured after the OPyC coating was burned off, and $6 \%$ to $38 \%$ of the particles showed flaws. The liquid gradient densities and coating rates of the OPyC ranged from 1.79 to $1.98 \mathrm{Mg} / \mathrm{m}^{3}$ and 5.3 to $9.0 \mu \mathrm{m} / \mathrm{min}$. The measured surface-connected porosity (microporosity) was dependent upon the coating rate, except for the Ardiluted OPyC coating, and varied from 21 to $59 \mathrm{ml} \mathrm{Hg} / \mathrm{kg}$ of OPyC coating. The Ar-diluted OPyC had a microporosity of $48 \mathrm{ml} / \mathrm{kg}$ OPyC, and a coating rate of $5.3 \mu \mathrm{m} / \mathrm{min}$, while the $\mathrm{H}_{2}$-diluted batches had values of $<28 \mathrm{ml} / \mathrm{kg}$ OPyC for the same coating rates. These $\mathrm{Hg}$ intrusion values show that $\mathrm{H}_{2}$ dilution reduced the interconnecting porosity in the OPyC coating. The optical anisotropy ranged from 1.027 to $1.049 \mathrm{BAF}_{0}$ for the batches. As expected, the anisotropy increased with density and decreased with coating rate (Ref. 8).

The total coated-particle properties include density, impurities, coating defects, and heavy metal contamination. Particle density, measured by mercury porosimetry, is a bulk density, since the mercury does not penetrate the OPyC pores. The liquid gradient density is higher, since the liquid penetrates the larger surface-connected pores of the OPyC coating. The chemfcal impurities were low for all batches. The SiC-coating had more defects (burn-batch test) than specified $\left(\leq 1 \times 1.0^{-3}\right)$ for two of the eight batches. Table 3-11 shows that for five of the parent batches, the SiCcoating defect measurements were higher in the burn-visual examination than they were with the burn-leach technique. It is presumed that the liquidleach method did not always leach all of the $\mathrm{ThO}_{2}$ kernel out of cracked Siccoated particles. Exposed heavy-metal Th contamination, measured by a liquid-leach method, was less than specified $\left(\leq 1 \times 10^{-4}\right)$ for six of the eight batches. The FGR specification of $\leq 3 \times 10^{-5} \mathrm{R} / \mathrm{B}$ (release/birth) was met for five of the batches. 
An interesting observation was that the FGR appeared to correlate somewhat with the defective SiC-coating fraction. The intact-OPyC coating of the defective SiC-coated particles may be permeable to the fission gases. Further supporting this permeability is the finding that the FGR tends to be higher in defective SiC-coated batches that have an OPyC coating deposited at higher coating rates. Kaae has reported (Ref. 13) that increasing the coating rate increased the surface-connected porosity of the pyrocarbon, which affects the permeability. The high SiC-coating defects in some batches will not affect the results of capsule HT-34, since any defective particles (determined by $\mathrm{X}$-radiography) were removed from the approximately 140 particles of each batch inserted in the capsule.

\subsubsection{Capsule Specimens}

The capsule specimens for HT-34 were selected from the parent batches described in Section 3.4.1; therefore, they reflect similar general properties. Complete characterization ( $R / B$, chemical analysis, etc.) was done on the parent batches. Many of these tests were not duplicated on the actual capsule specimens, since the only major change was in particle dimensions.

Table 3-12 gives actual HT-34-capsule-specimen density and dimensional measurements. A summary of the properties of the capsule samples is presented in Table 3-13. The properties measured on the capsule samples are compared to the parent batch properties in Table 3-14. As expected, the standard deviation of the buffer thickness and the total particle diameter were significantly reduced in the capsule samples. The table shows how the buffer coating and the other coating thicknesses changed for low-, mean-, and high-density fractions. The dimensions of the mean-density fractions were in good agreement with those of the parent batches. 


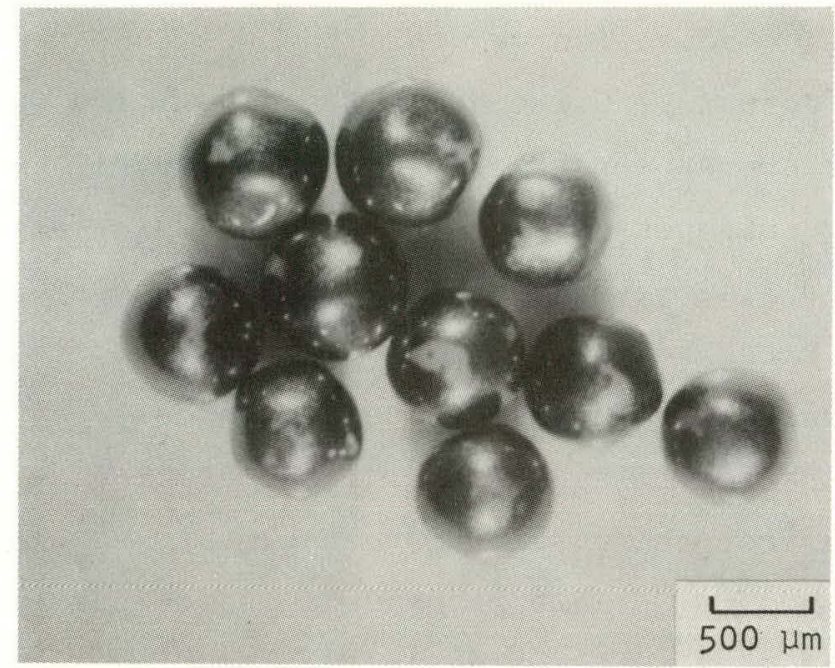

N77003

STEREO VIEW OF SiC-COATED PARTICLES

SHOWING GOLD SPOTS OR FLAWS

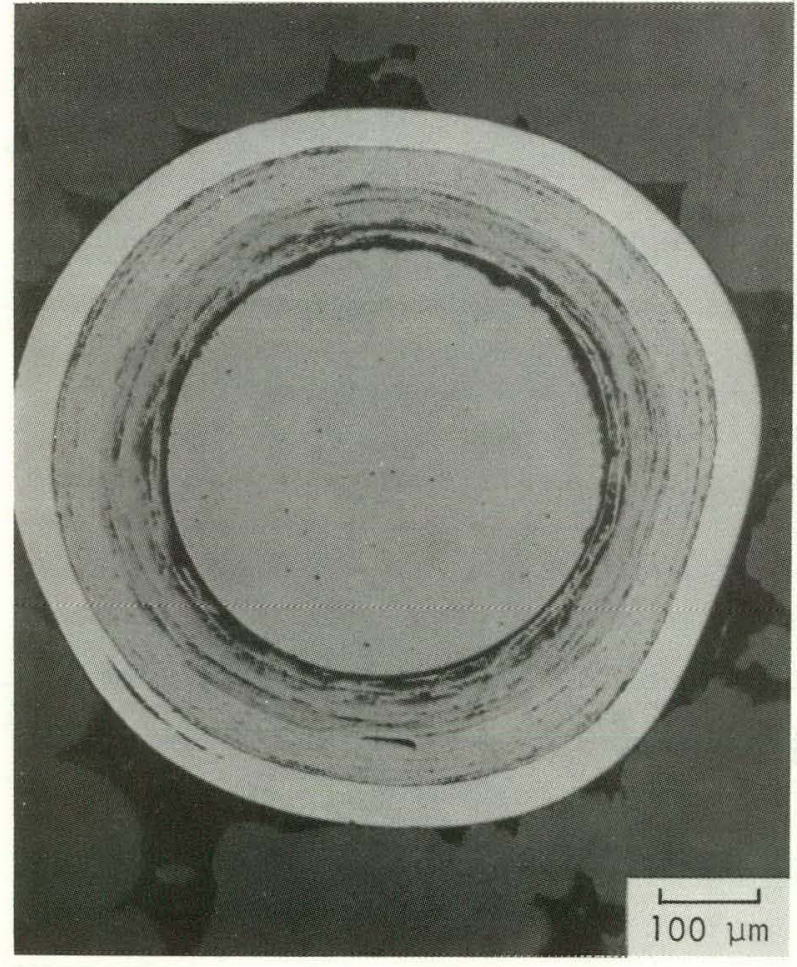

MP77001-11

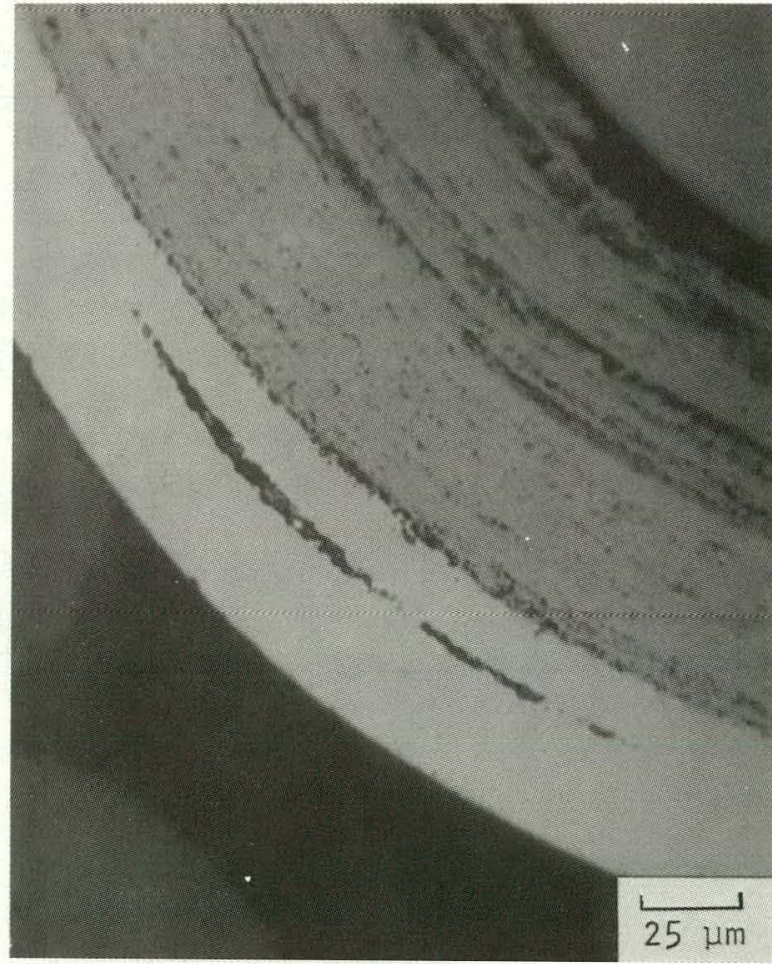

MP77001-10

METALLOGRAPHIC CROSS SECTION

Fig. 3-1. Typical internal flaws in the SiC coating of a TRISO-ThO 2 particle 

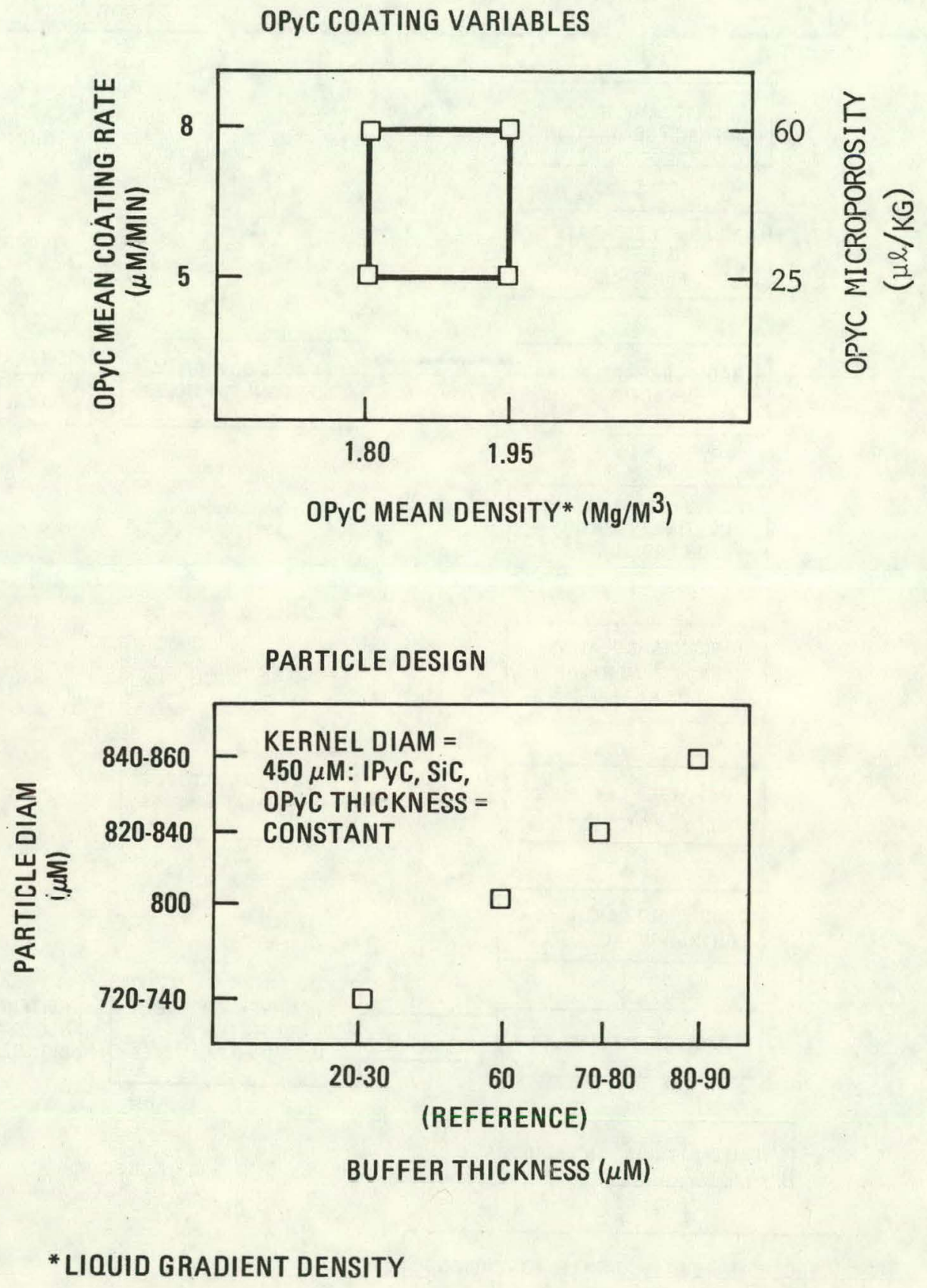

Fig. 3-2. Design test matrix of TRISO $\mathrm{ThO}_{2}$ particles for capsule H'-34. Particles made in 240-mm-diam coater using $\mathrm{H}_{2}$ dilution for the OPyC deposition 


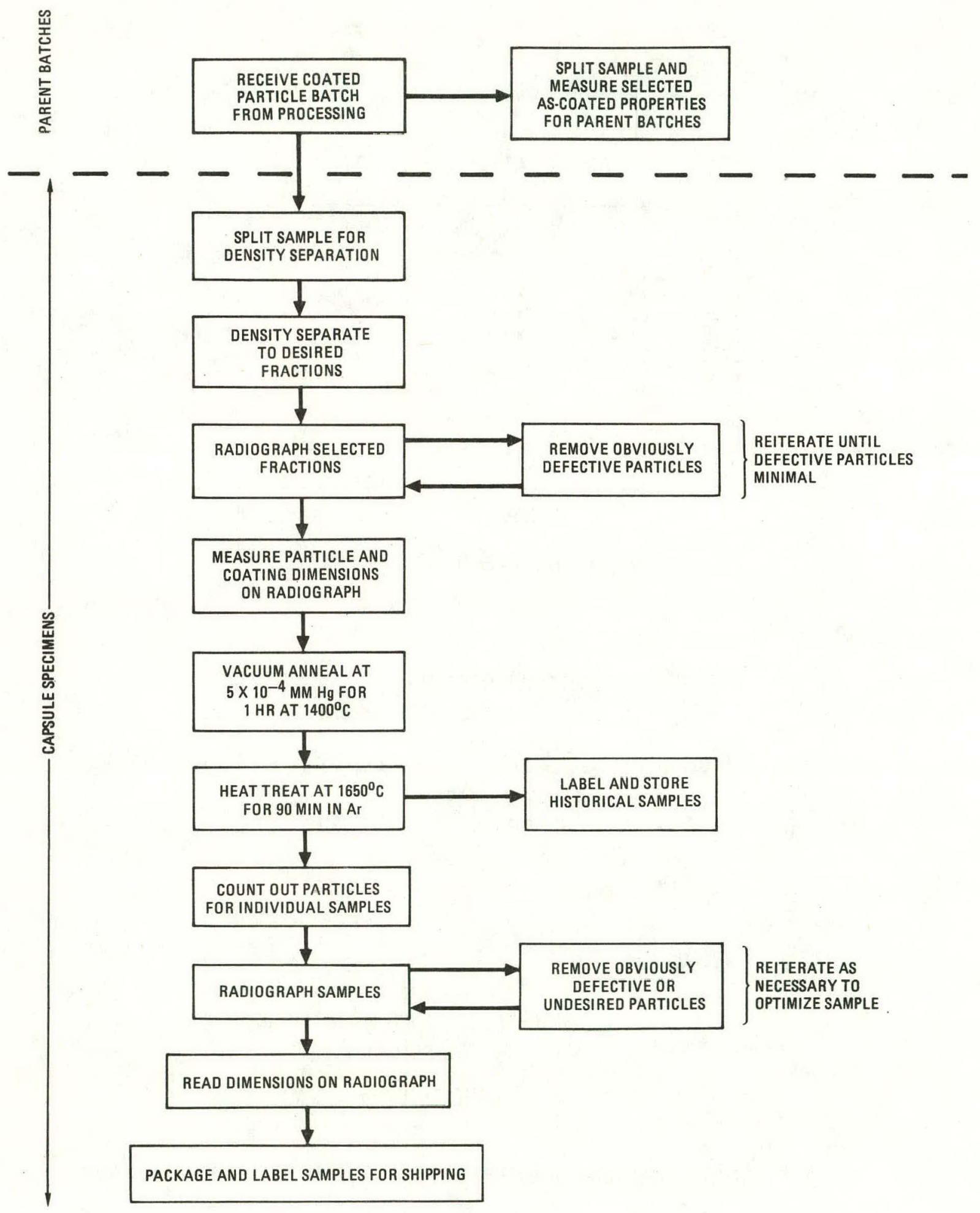

Fig. 3-3. Selection and characterization procedure for HT-34-capsule specimens 


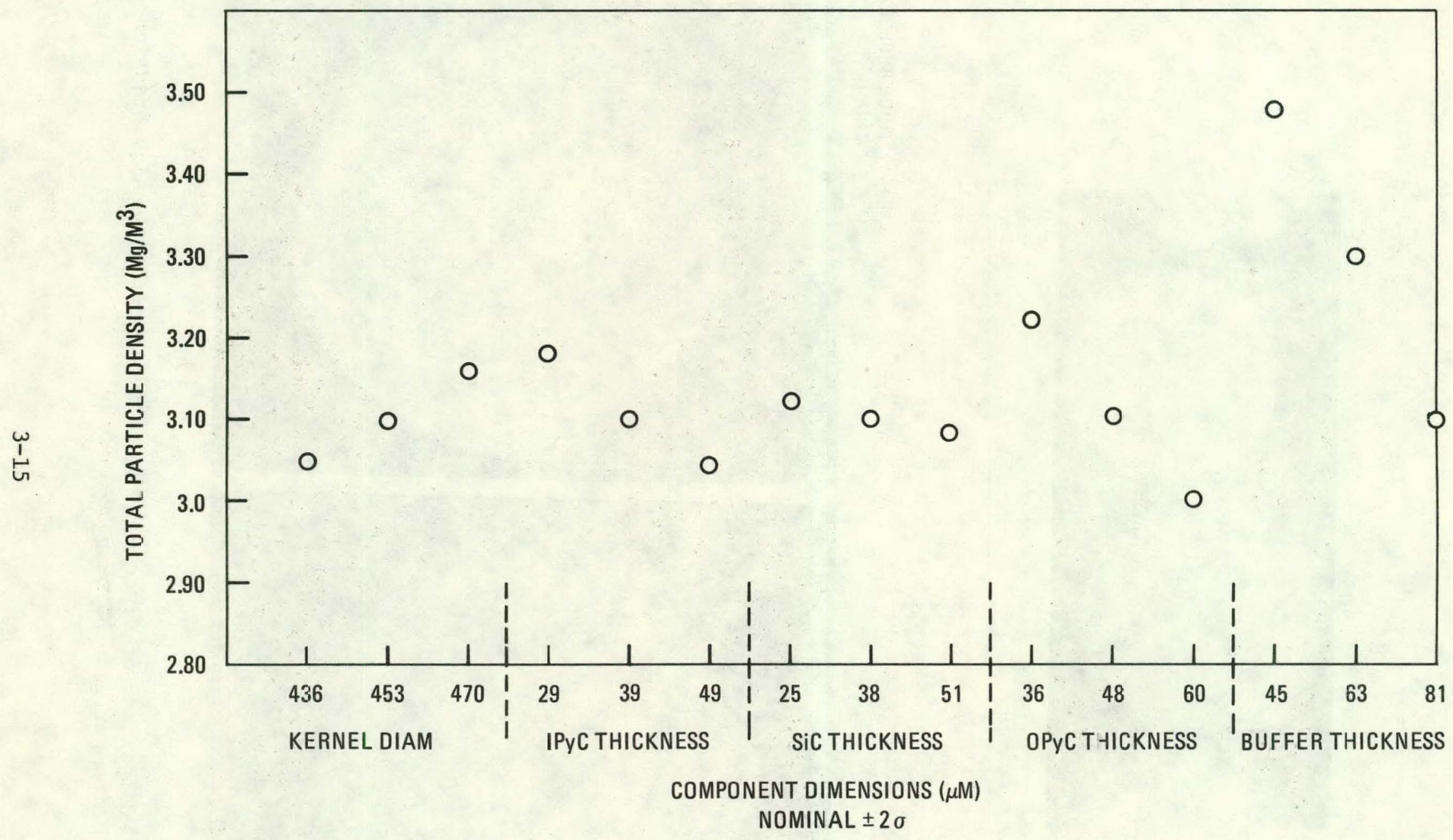

Fig. 3-4. Influence of various particle components on total density (one component varied at a time; other components kept at nominal value) 


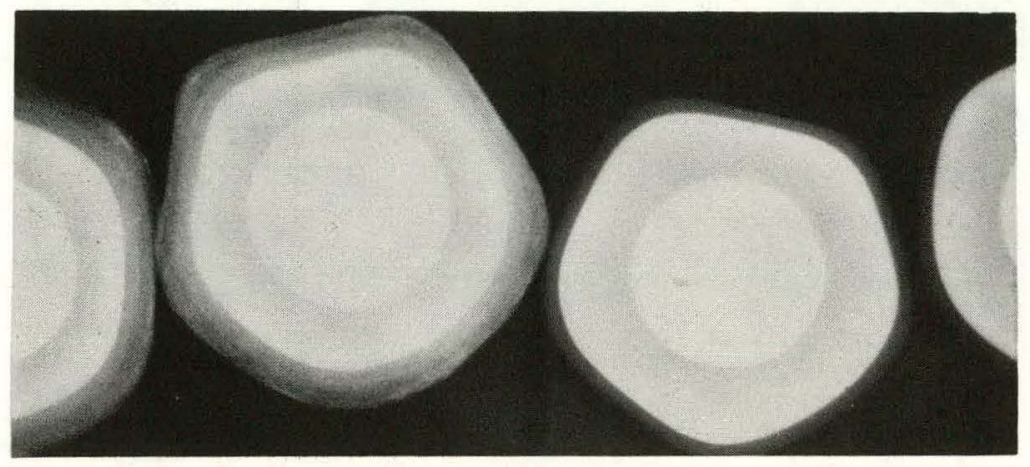

THIN BUFFER

THICK POROUS

THICK BUFFER

$\mathrm{OPyC}$

THIN OPyC
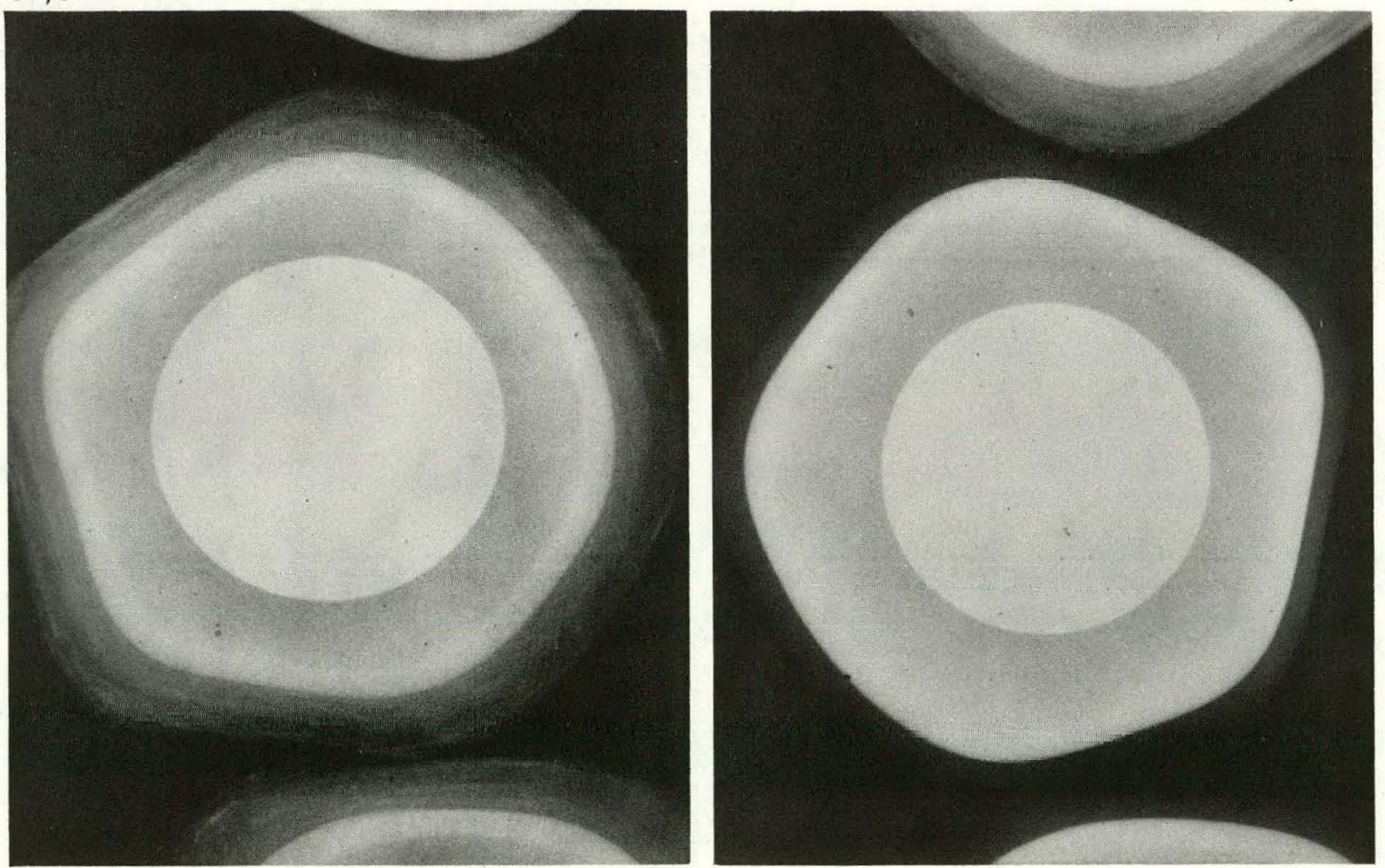

Fig. 3-5. Comparison of diverse particle types in low density fraction from particle batch 6252-14-0200 

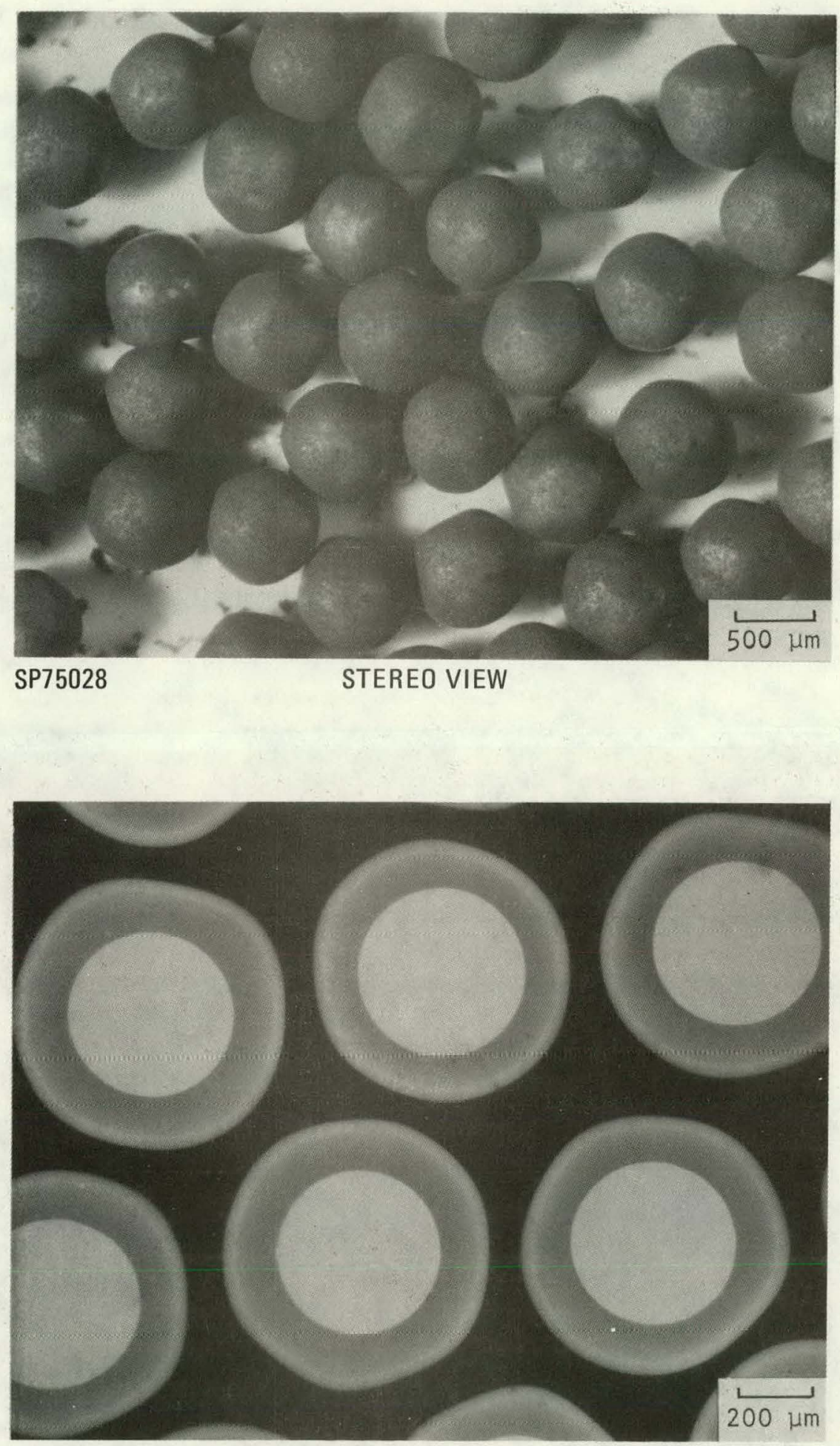

LB 307-2

X-RADIOGRAPH

Fig. 3-6. Representative photomicrographs of TRISO-coated $\mathrm{ThO}_{2}$ batch 625207-020 for capsule HT-34: stereo view and X-radiograph 


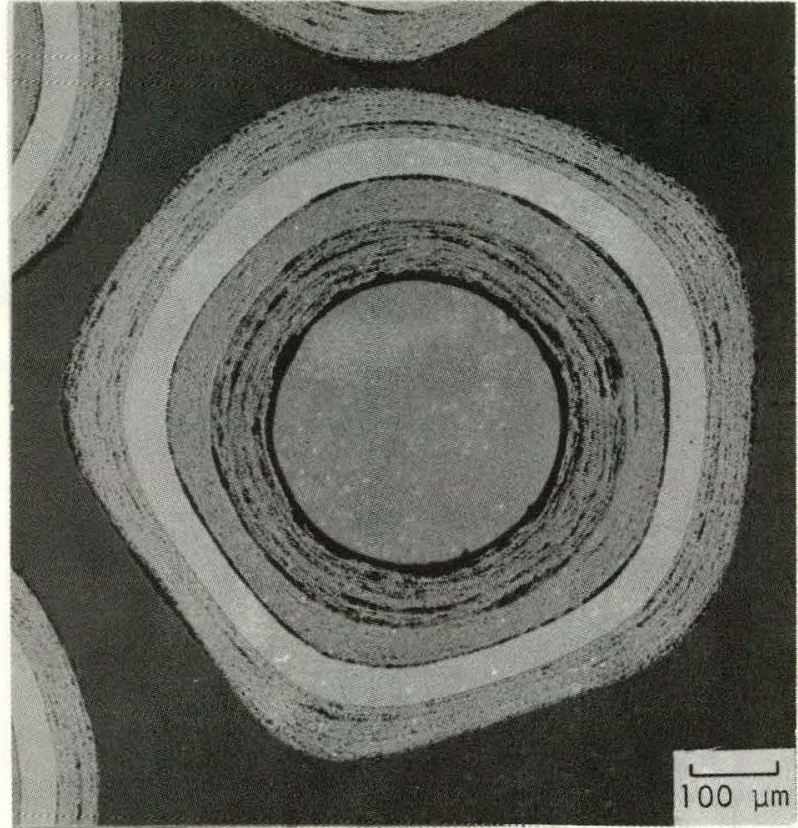

MP75044-1

BRIGHT FIELD

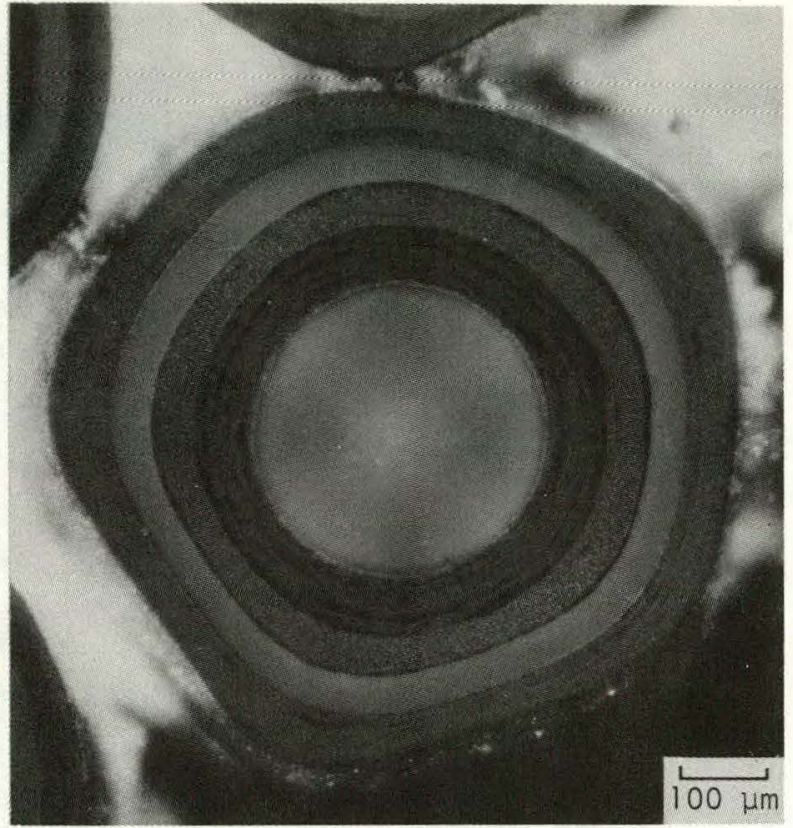

MP75044-2

POLARIZED LIGHT

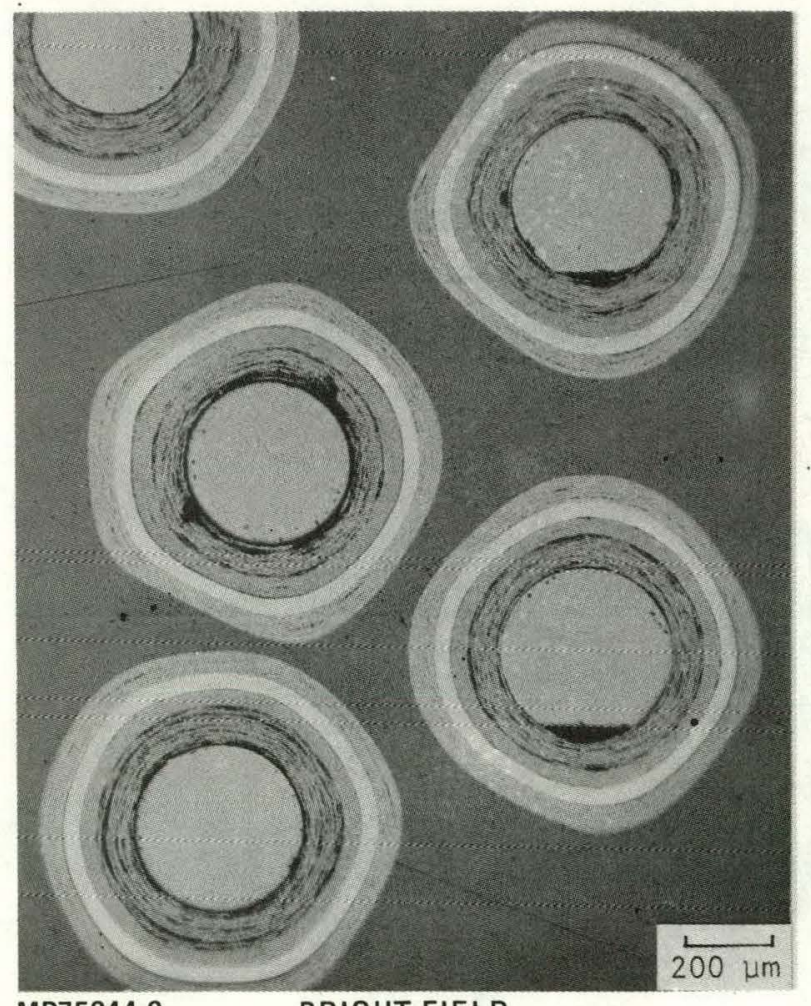

MP75044-6
BRIGHT FIELD

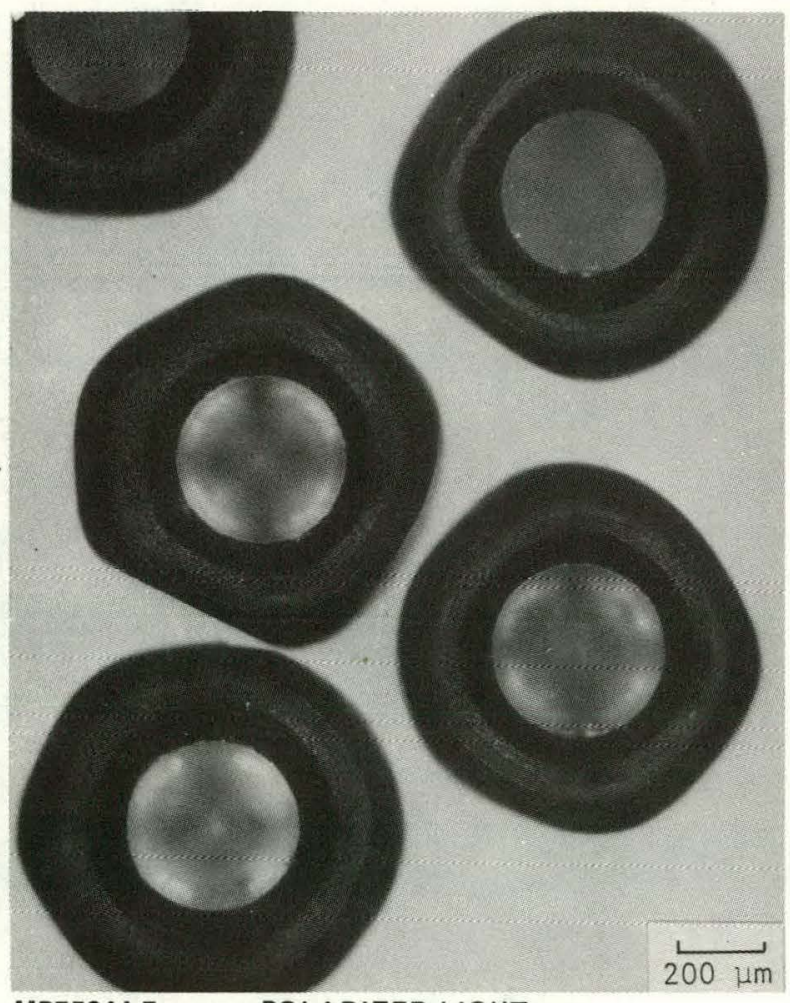

MP75044-7

POLARIZED LIGHT

Fig. 3-7. Representative photomicrographs of TRISO coated $\mathrm{ThO}_{2}$ batch 6252-07-020 for capsule HT-34: bright field and polarized light 

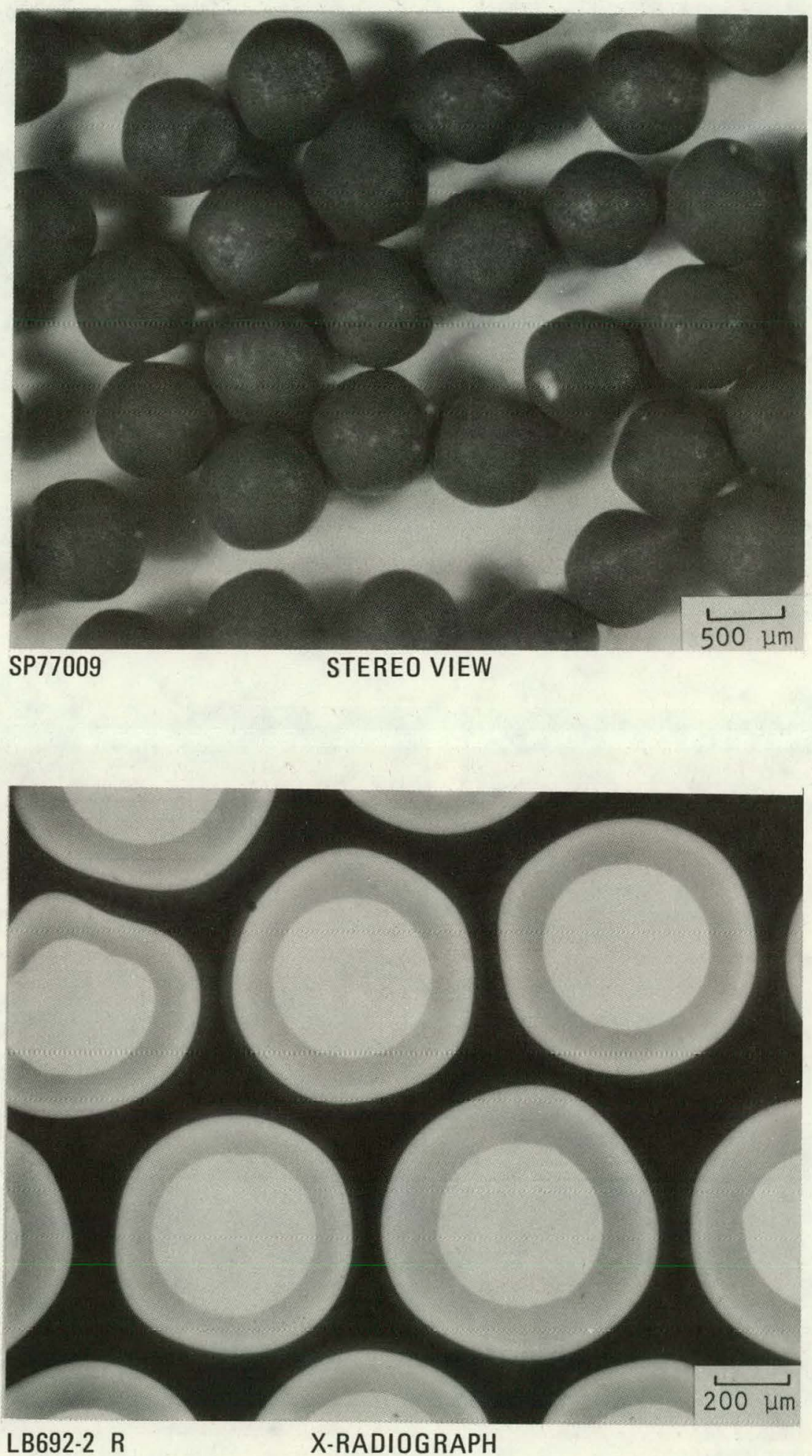

Fig. 3-8. Representative photomicrographs of TRISO-coated $\mathrm{ThO}_{2}$ batch 6252-13-010 for capsule HT-34: stereo view and X-radiograph 

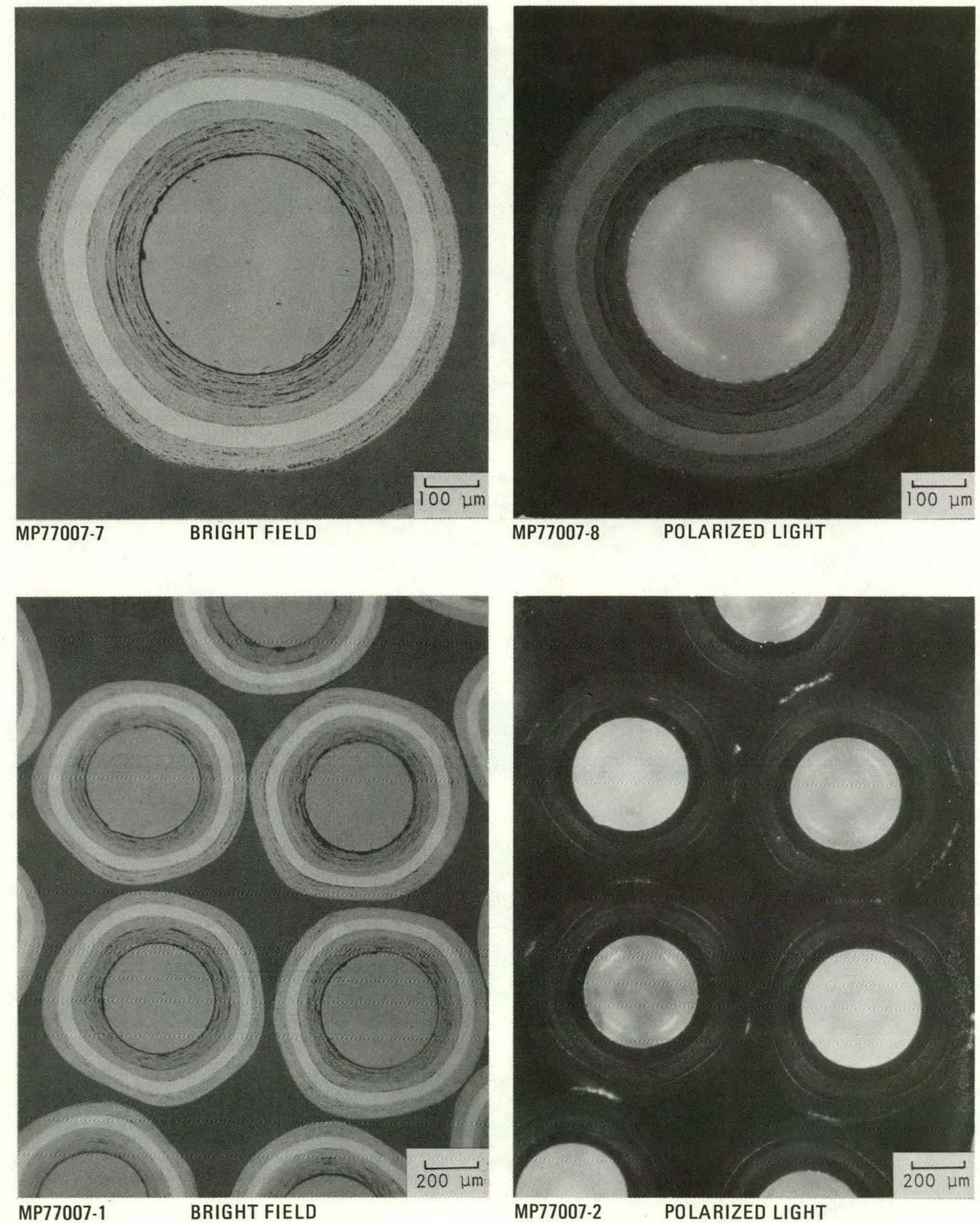

Fig. 3-9. Representative photomicrographs of TRISO-coated $\mathrm{ThO}_{2}$ batch 6252-13-010 for capsule HT-34: bright field and polarized light 

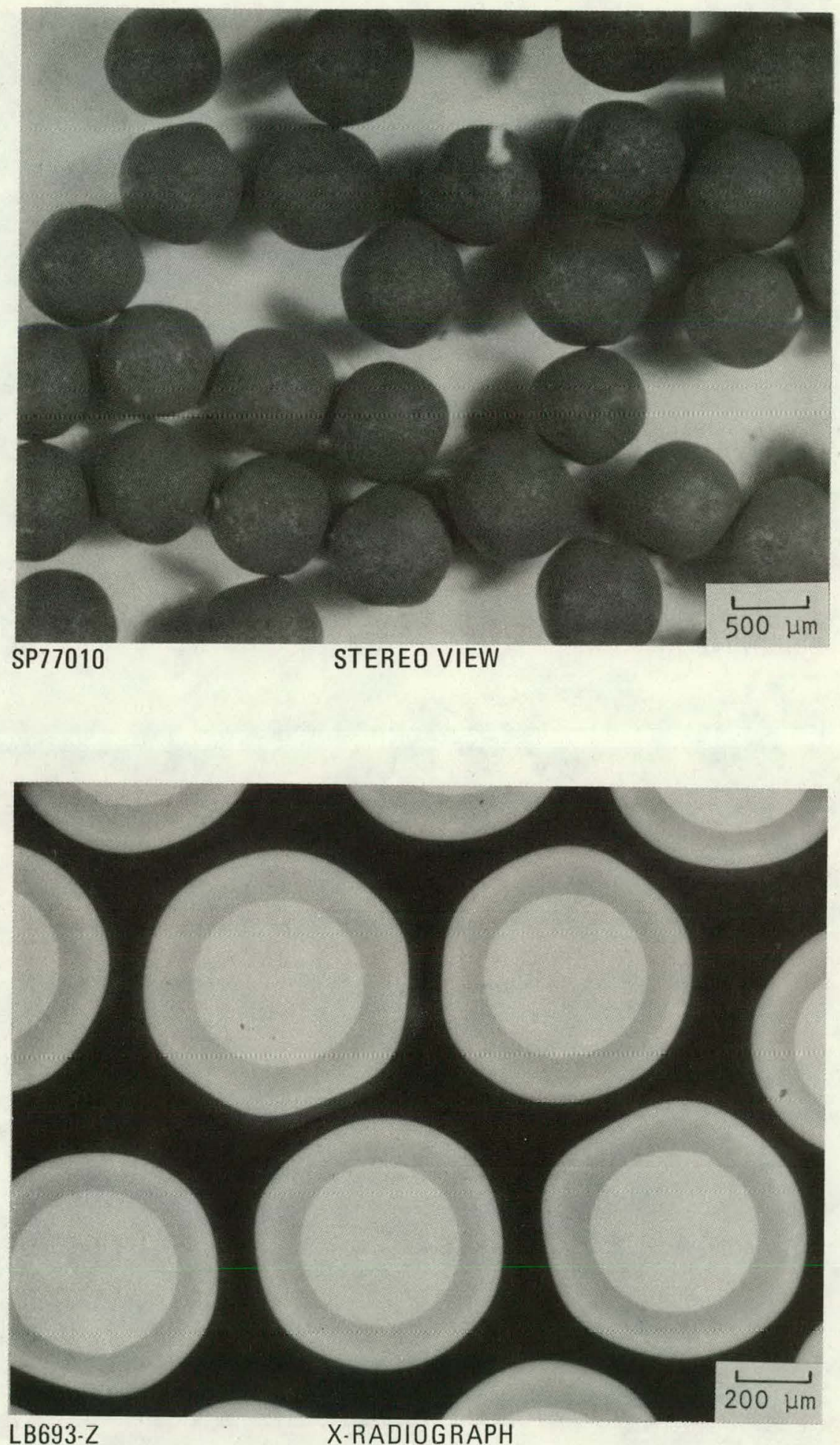

Fig. 3-10. Representative photomicrographs of TRISO-coated 'I'hO 2 batch 6252-14-020 for capsule HT-34: stereo view and X-radiograph 


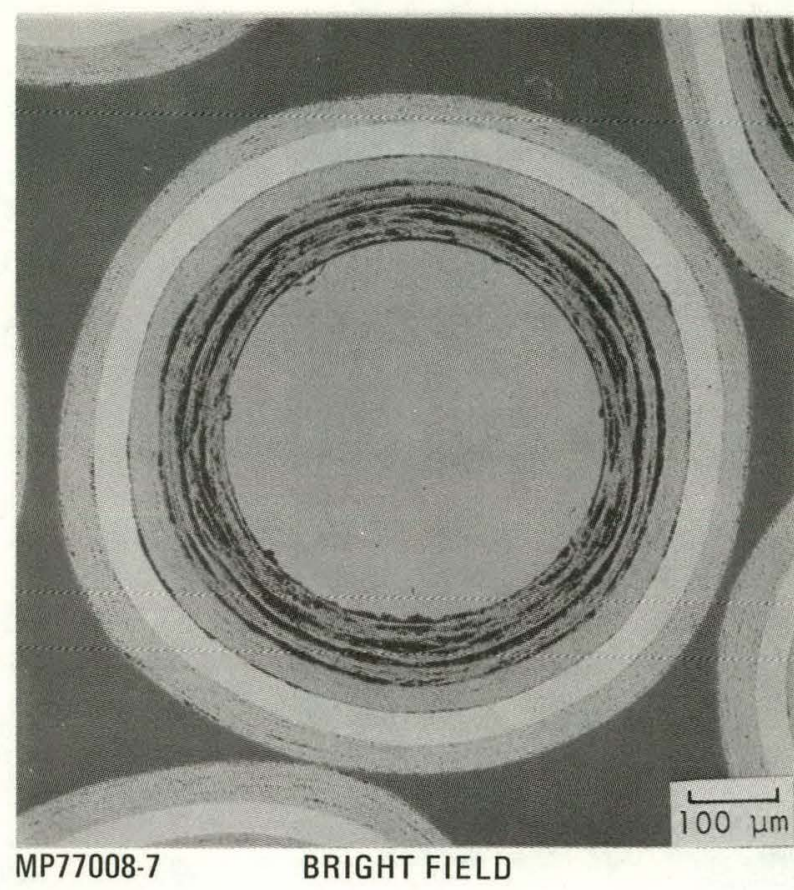

MP77008-7

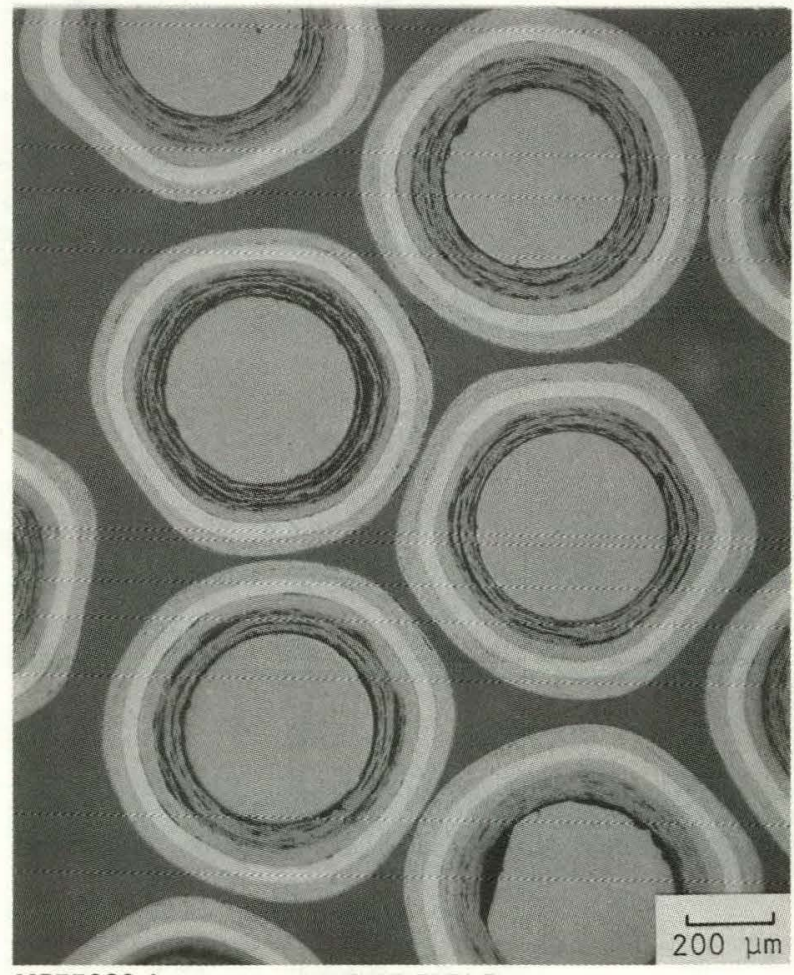

MP77008-1
BRIGHT FIELD

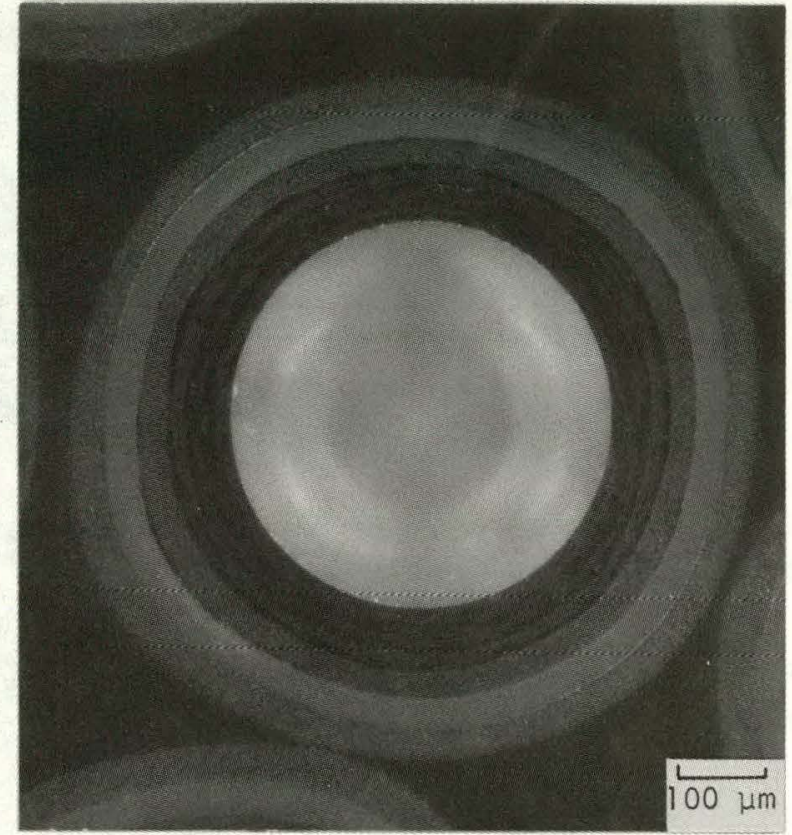

MP77008-8 POLARIZED LIGHT

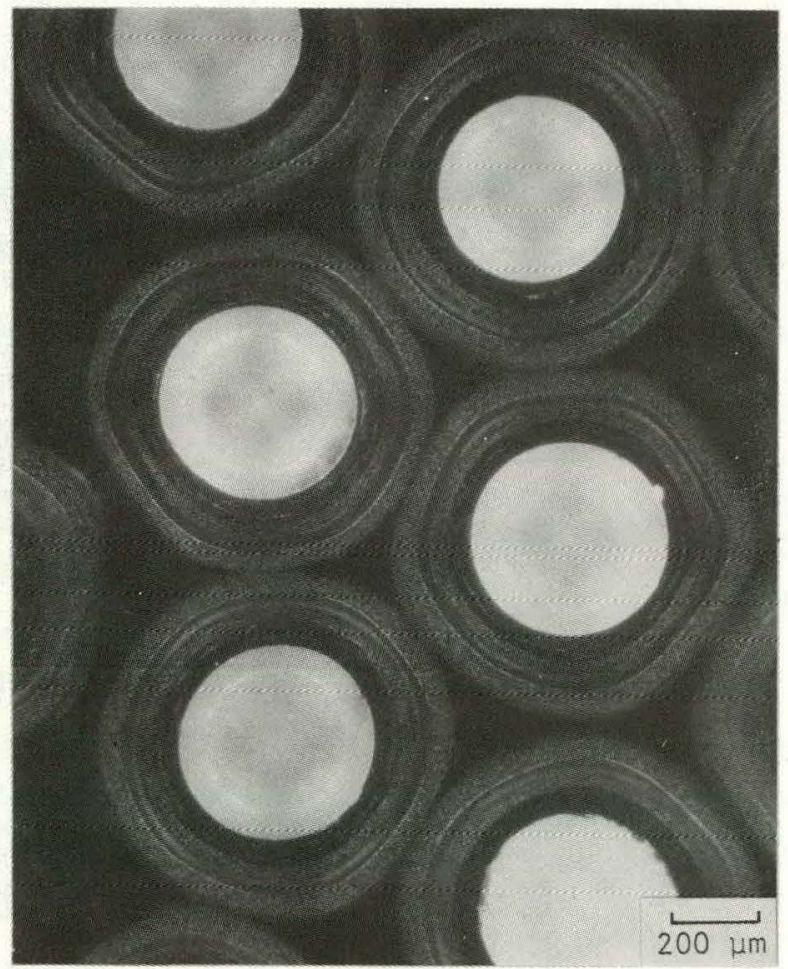

MP77008-2
POLARIZED LIGHT

Fig. 3-11. Representative photomicrographs of TRISO-coated $\mathrm{ThO}_{2}$ batch 6252-14-020 for capsule HT-34: bright field and polarized light 

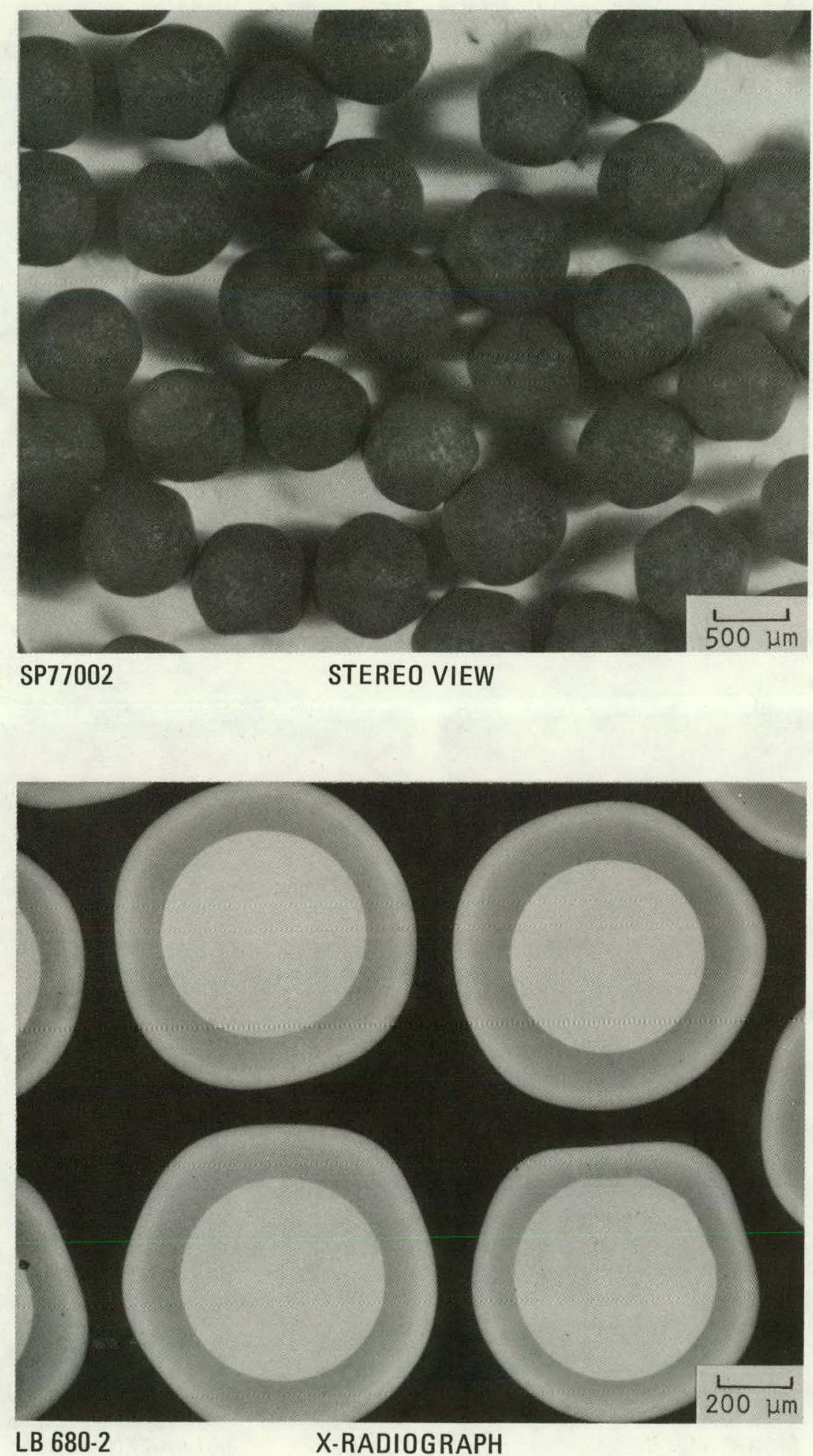

F'ig. 3-12. Representative photomicrographs of TRISO-coated $\mathrm{ThO}_{2}$ batch 6252-14-020 for capsule HI-34: stereo view and X-radiograph 

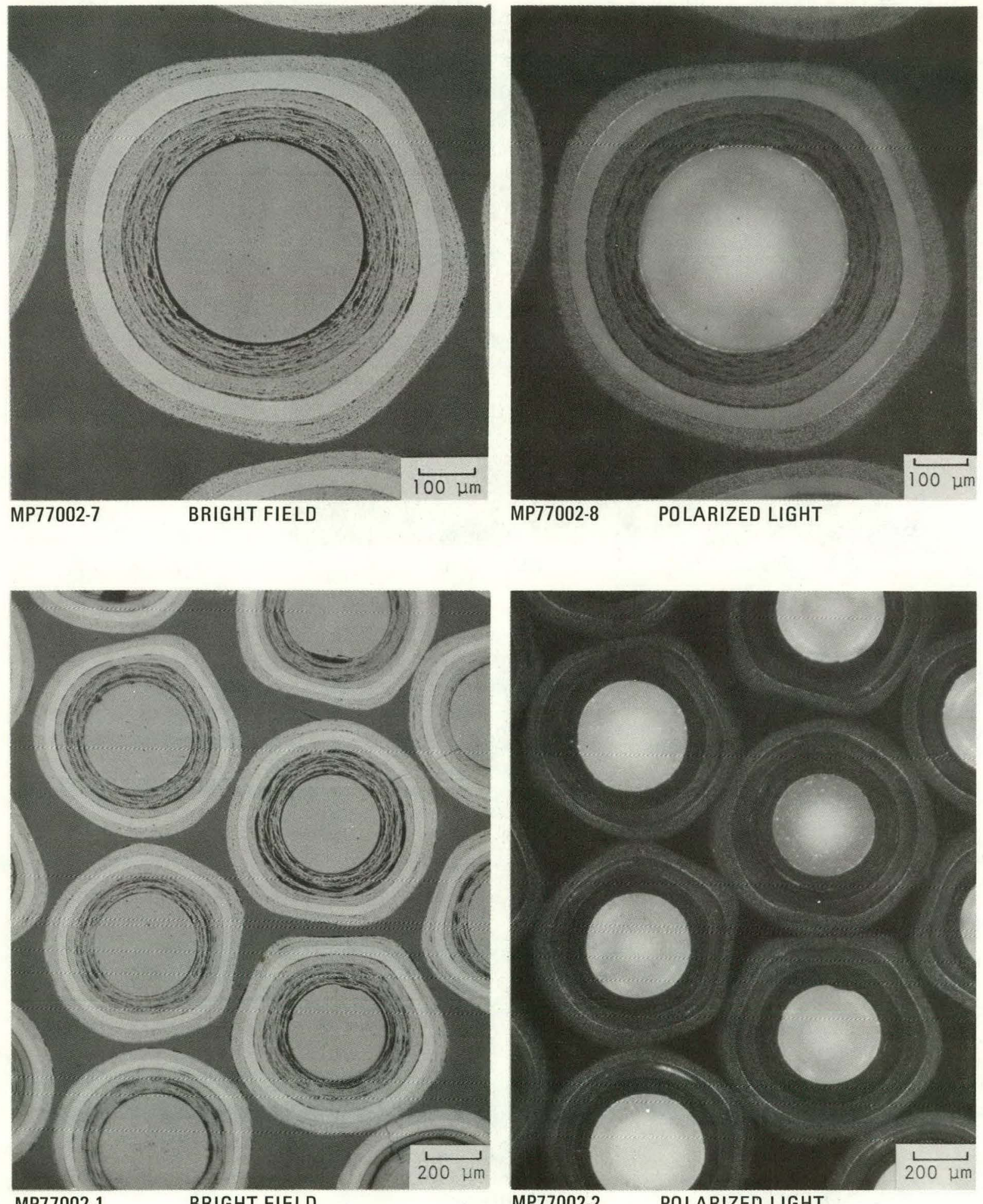

Fig. 3-13. Representative photomicrographs of TRISO-coated Th02 batch 6252-14-020 for capsule HT-34: bright field and polarized light 

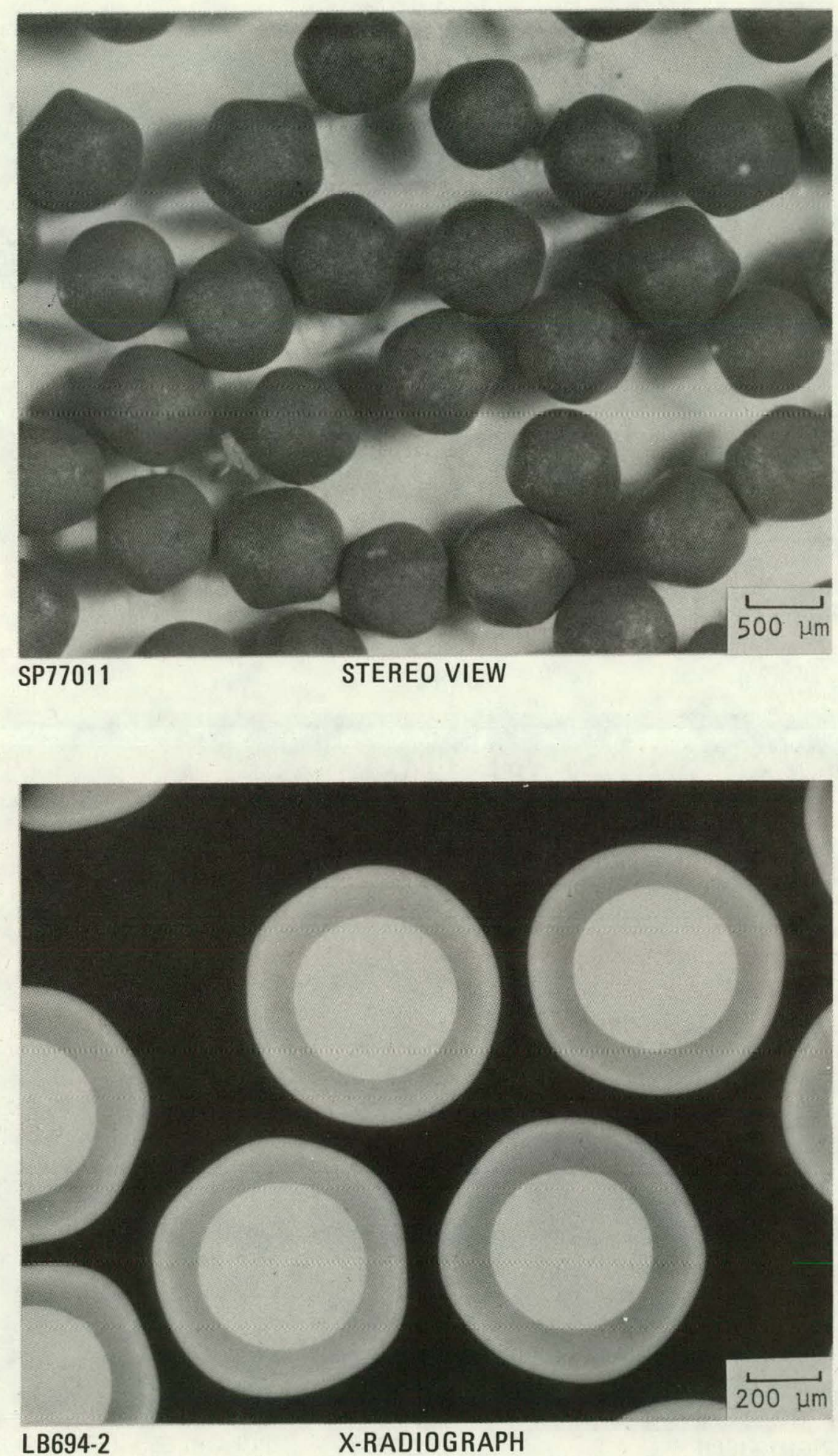

Fig. 3-14. Representative photomicrographs of TRISO-coated $\mathrm{ThO}_{2}$ batch 6252-15-020 for capsule HT-34: stereo view and X-radiograph 


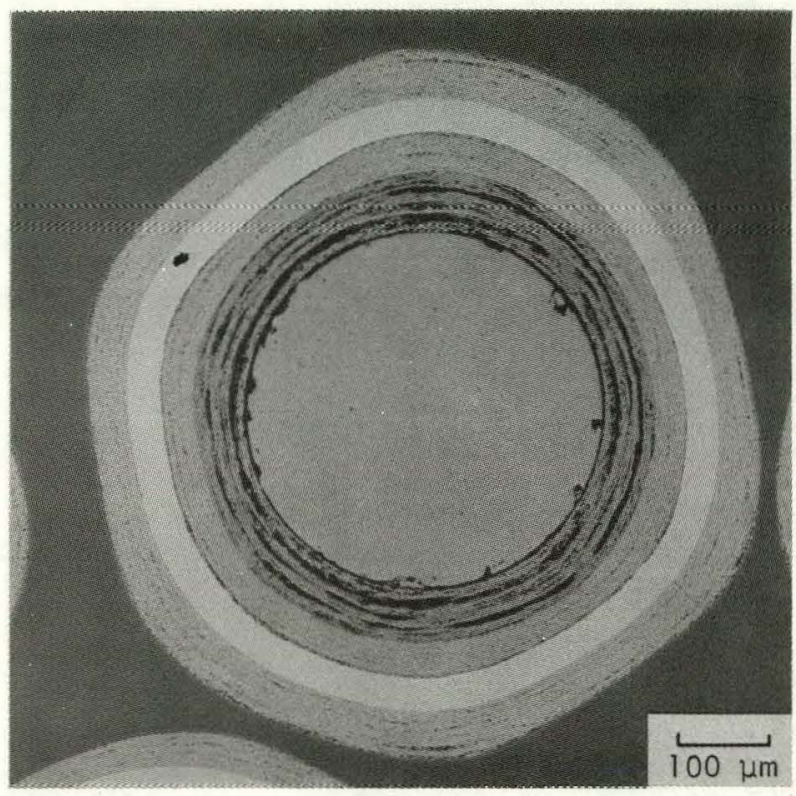

MP77009-7

BRIGHT FIELD

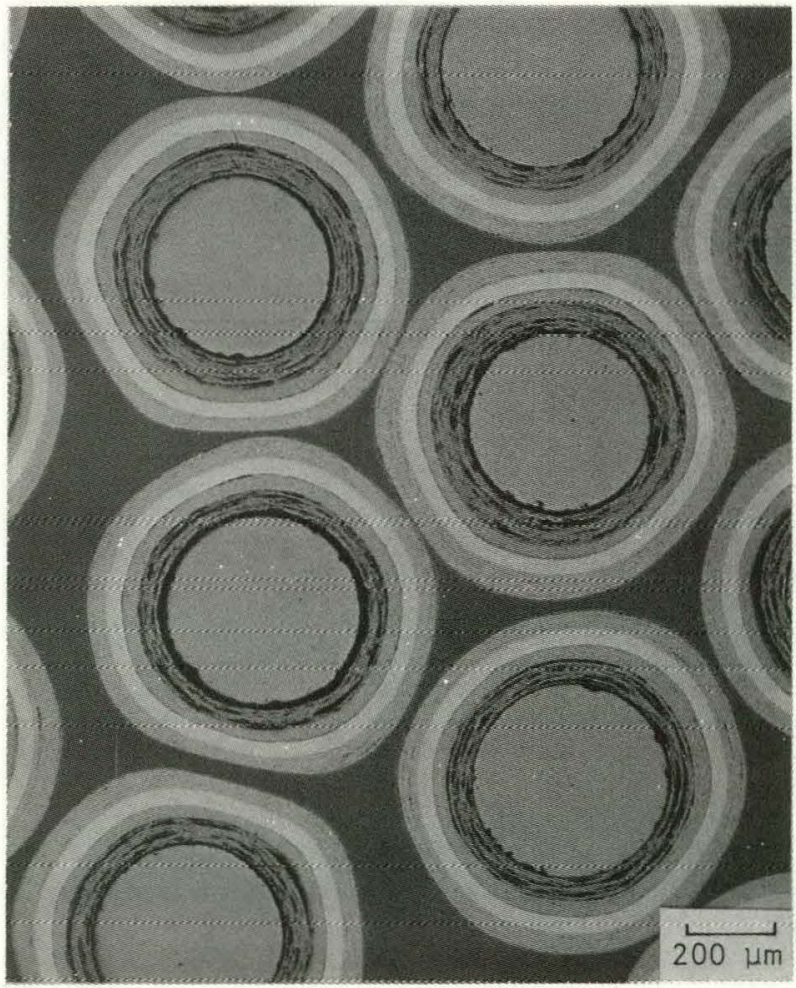

MP77009-1
BRIGHT FIELD

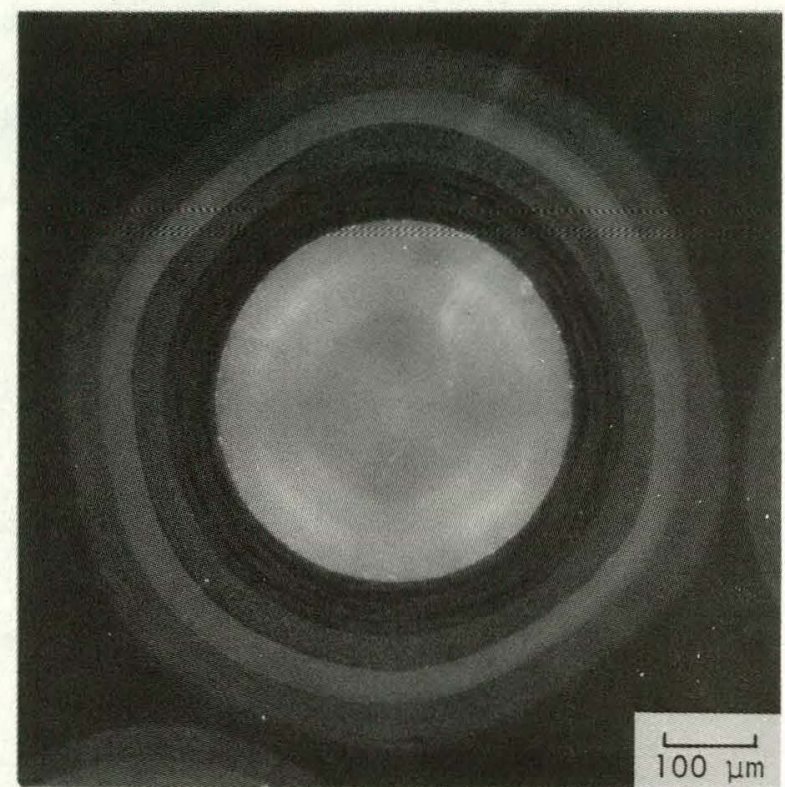

MP77009-8 POLARIZED LIGHT

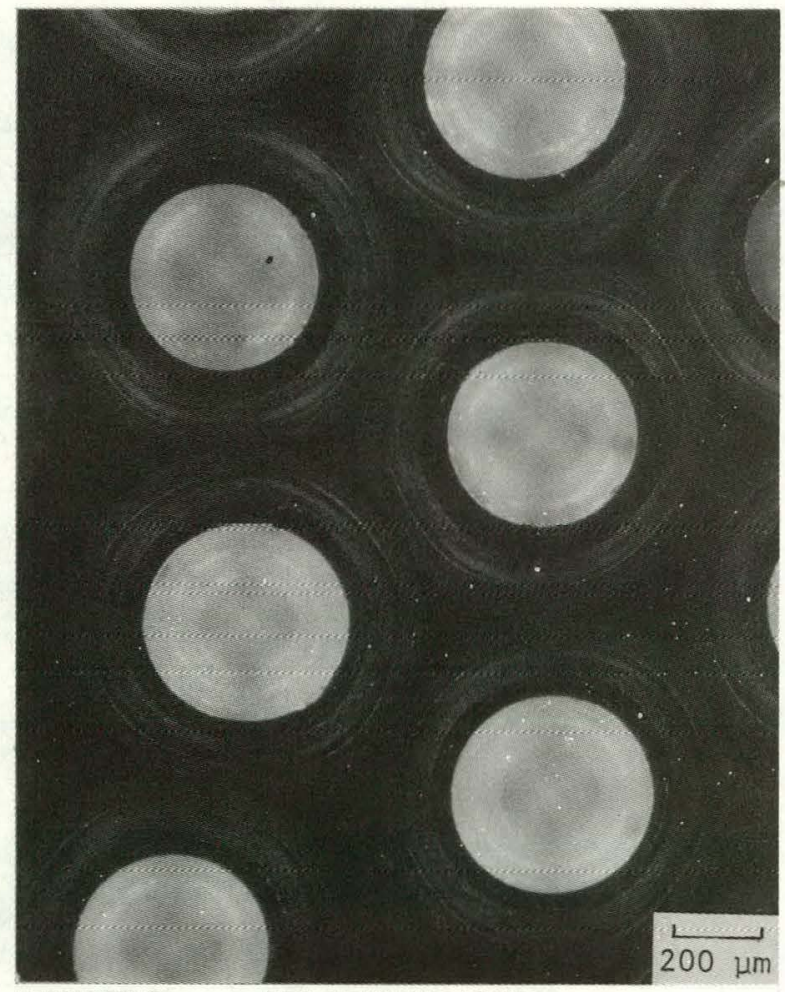

MP77009-2

POLARIZED LIGHT

Fig. 3-15. Representative photomicrographs of TRISO-coated $\mathrm{ThO}_{2}$ batch 6252-15-020 for capsule HT-34: bright field and polarized light 

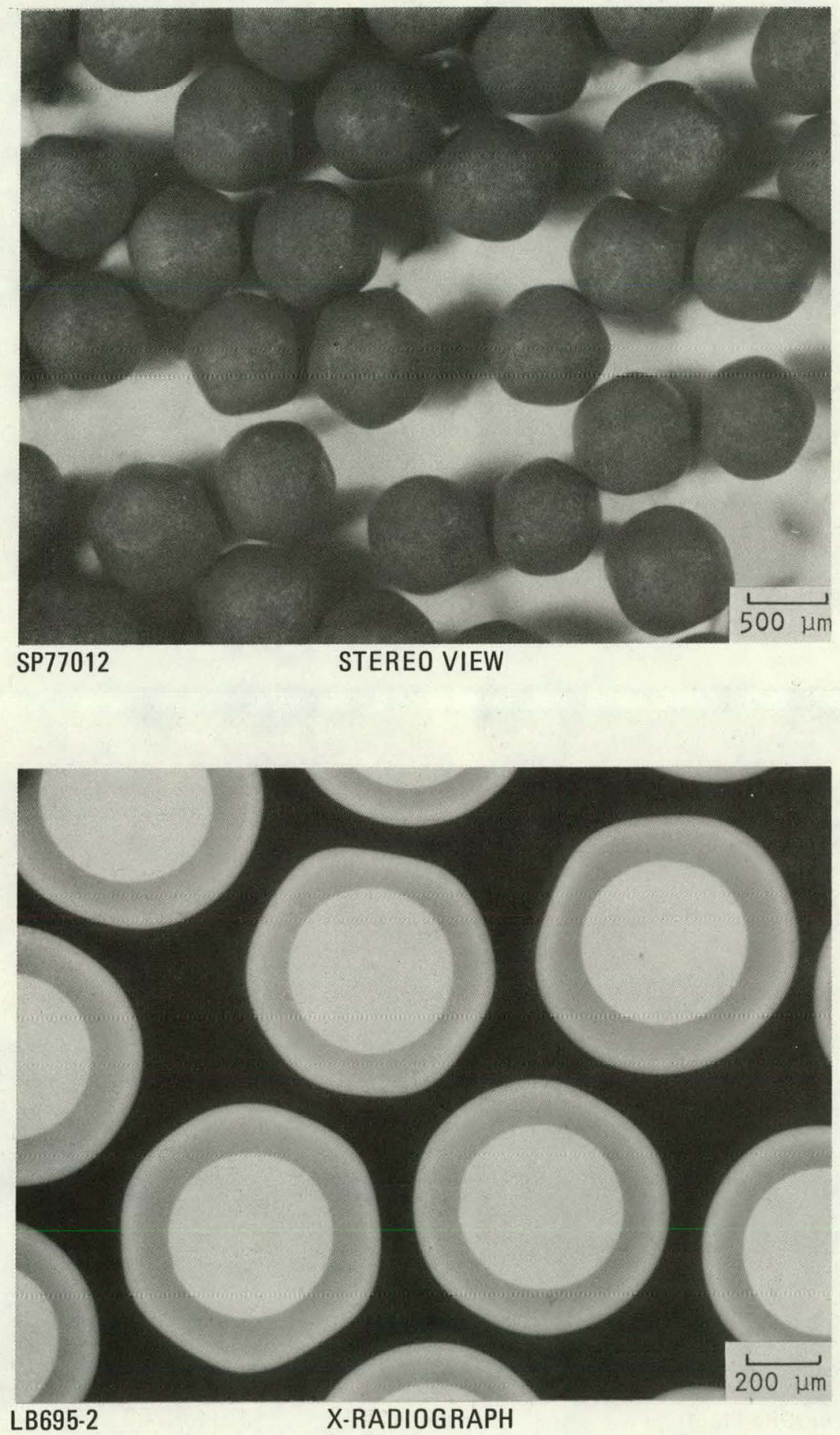

Fig. 3-16. Representative photomicrographs of TRISO-coated 'l'hU 2 batch 6252-16-010 for capsule HT-34: stereo view and X-radiograph 


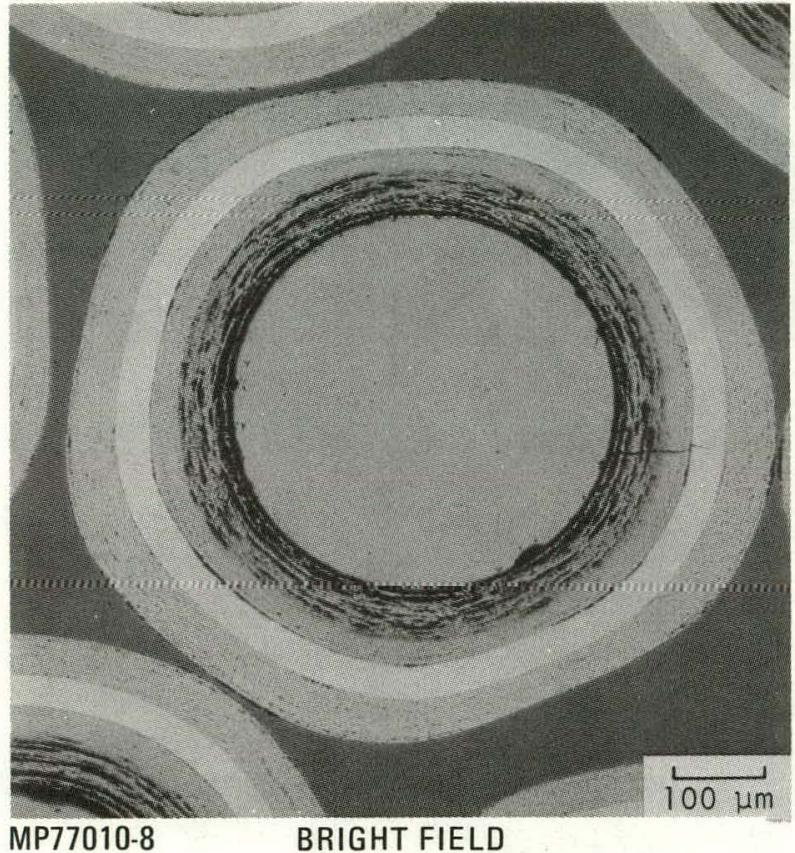

MP77010-8

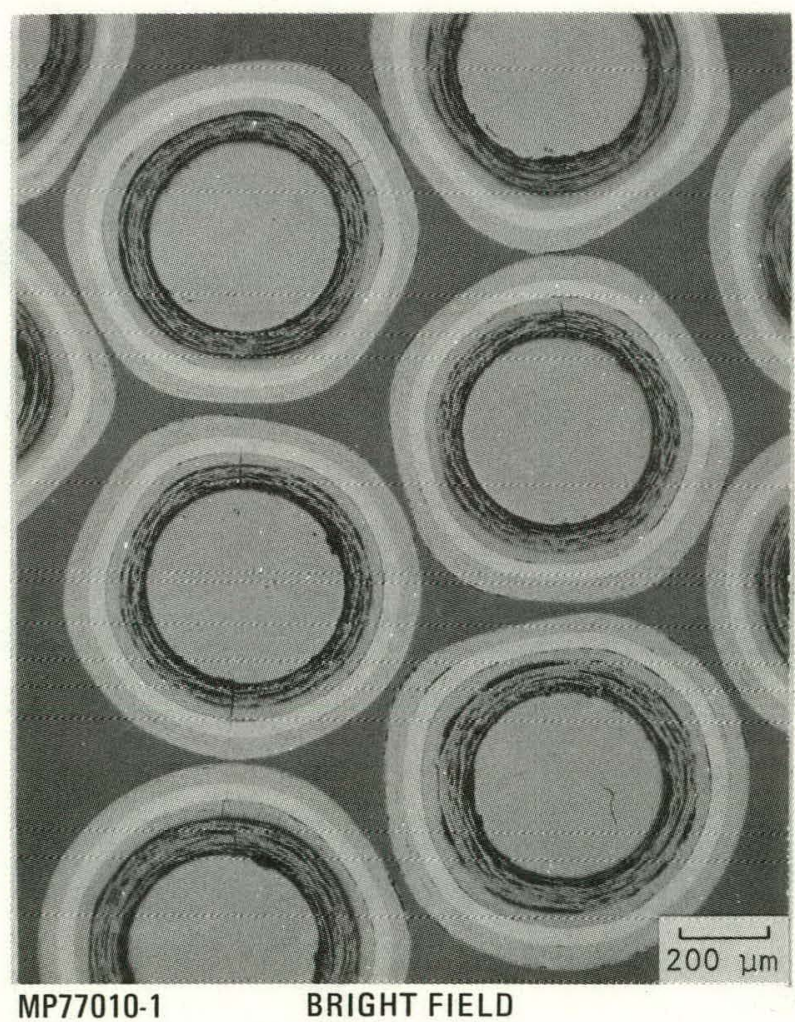

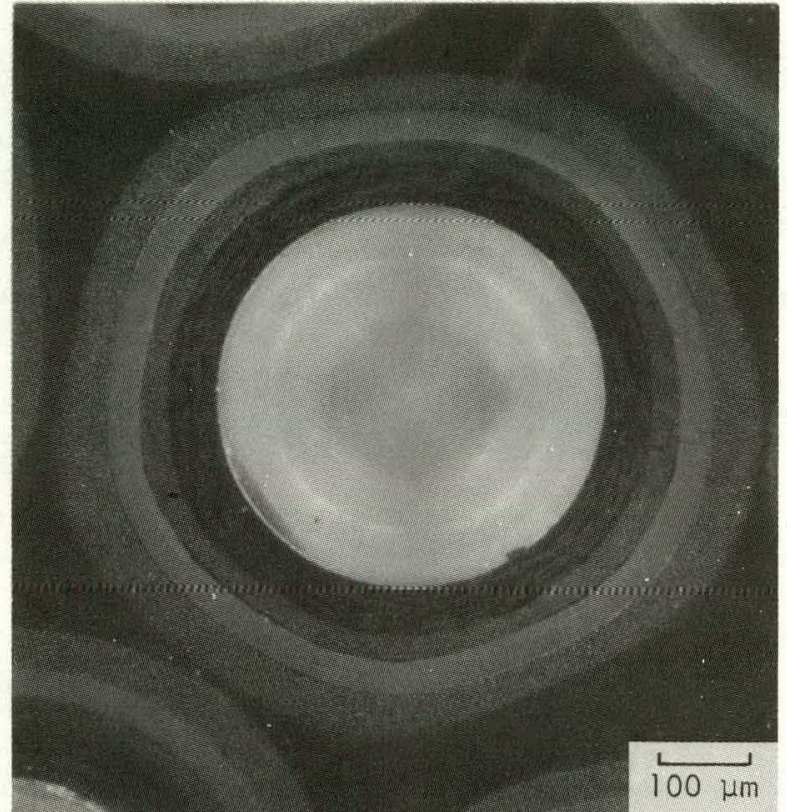

MP77010-7 POLARIZED LIGHT

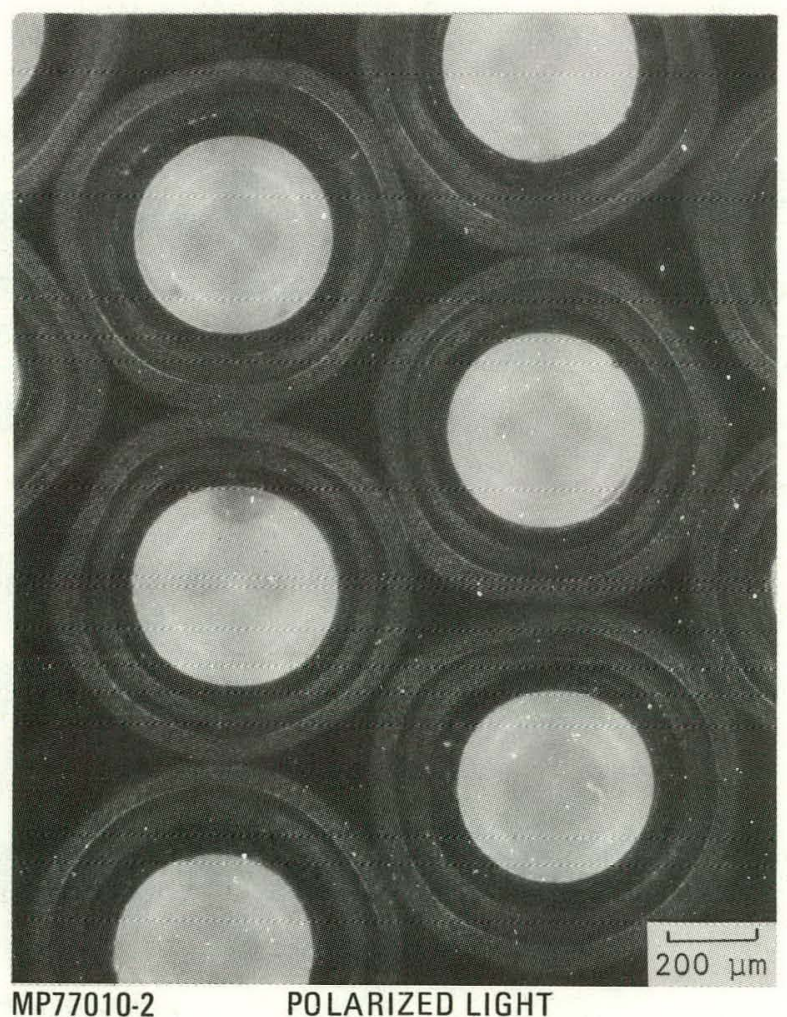

MP77010-2

POLARIZED LIGHT

Fig. 3-17. Representative photomicrographs of TRISO-coated $\mathrm{ThO}_{2}$ batch 6252-16-010 for capsule HT-34: bright field and polarized light 

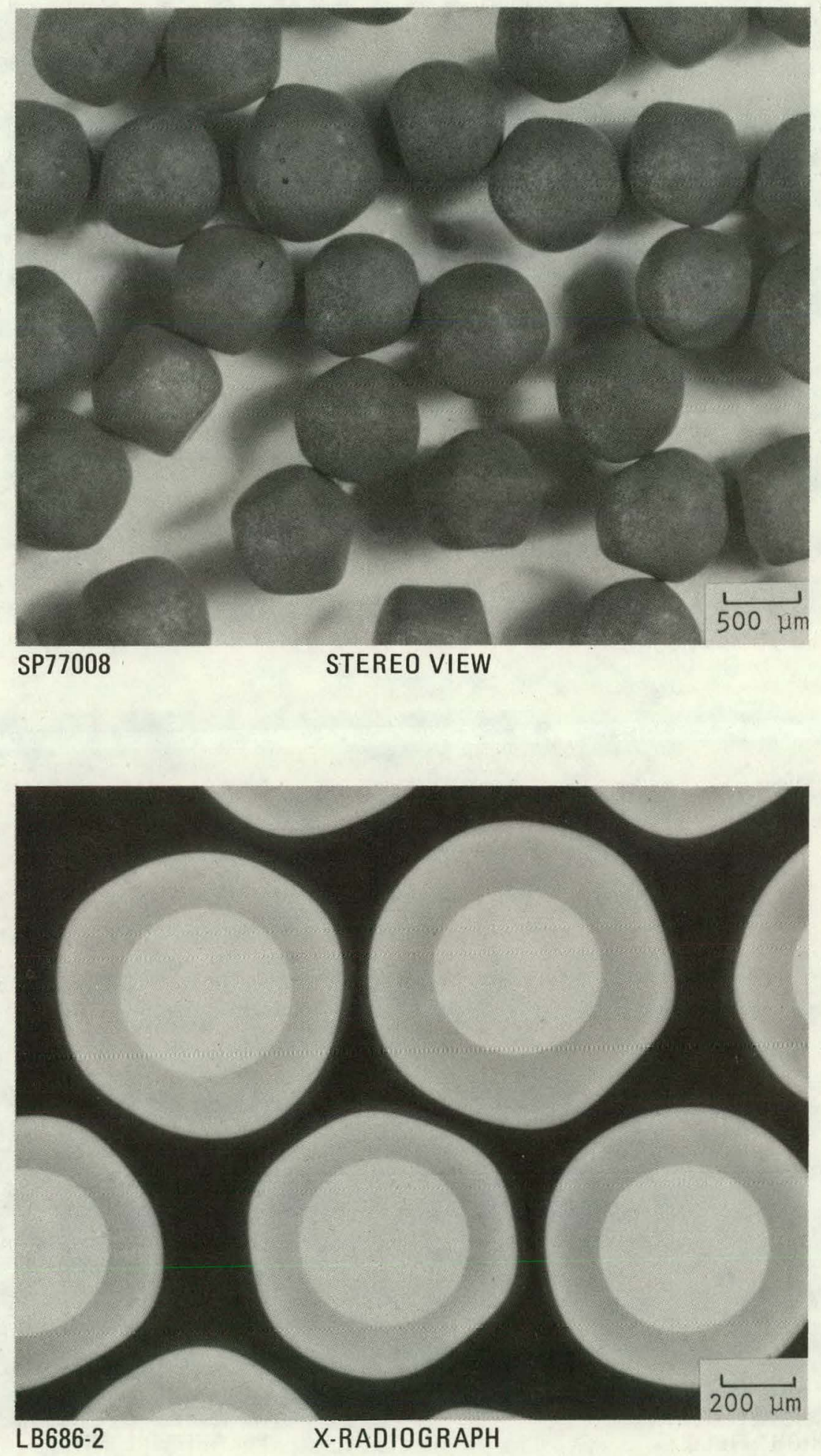

Fig. 3-18. Representative photomicrographs of TRISU-coated $\mathrm{ThO}_{2}$ batch 6252-17-010 for capsule HT-34: stereo view and X-radiograph 


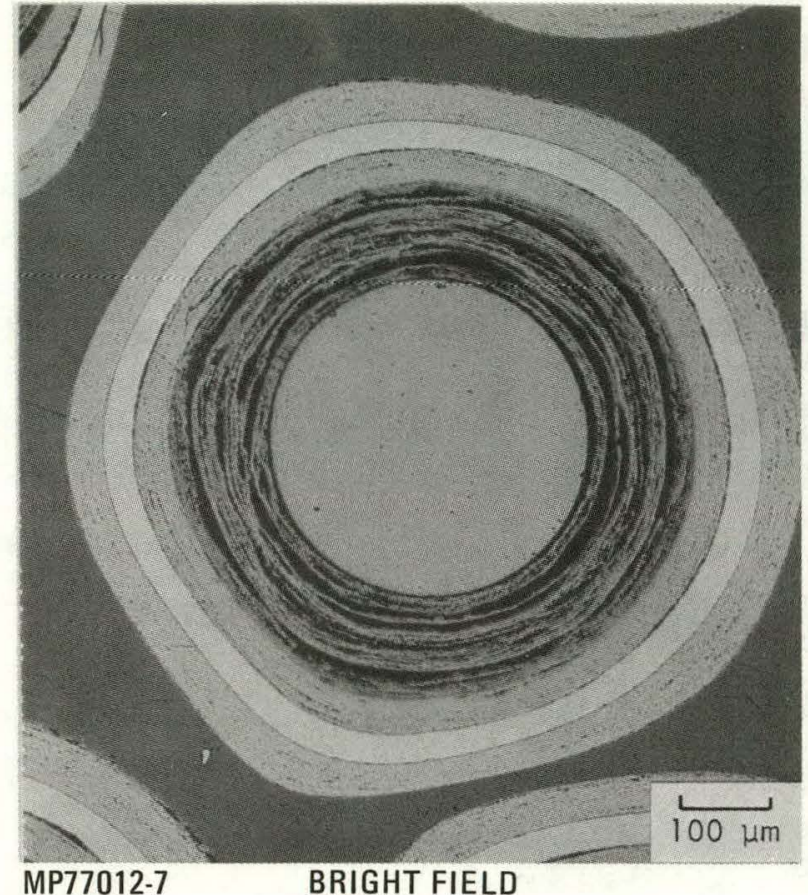

MP77012-7

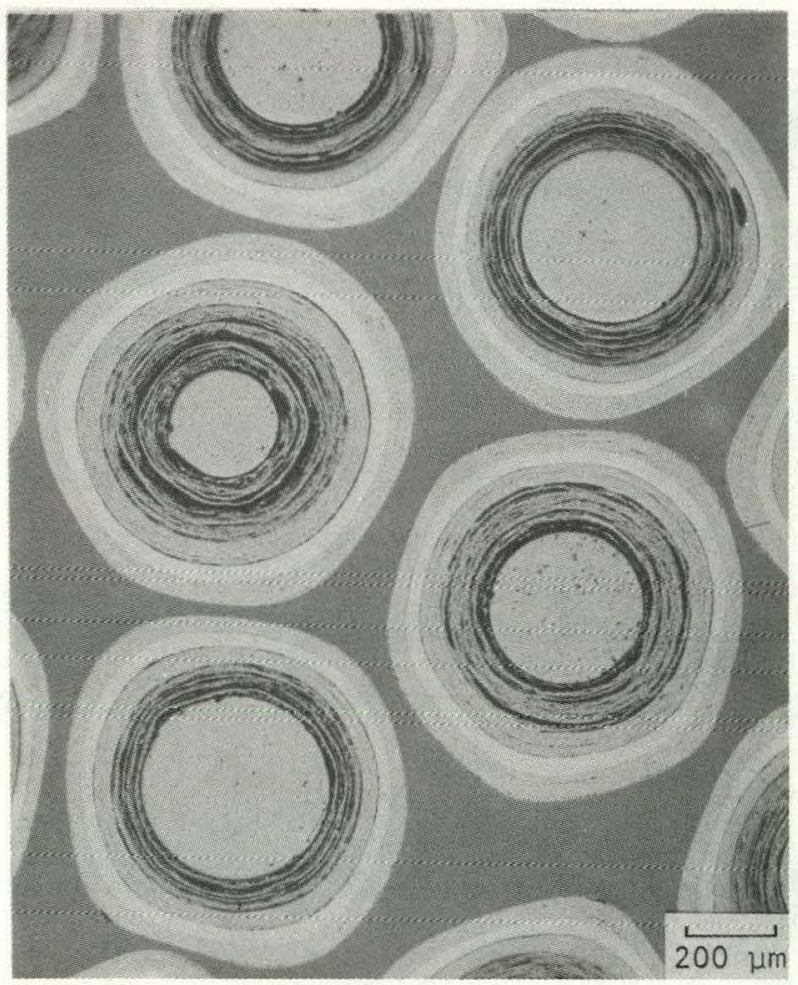

MP77012-1

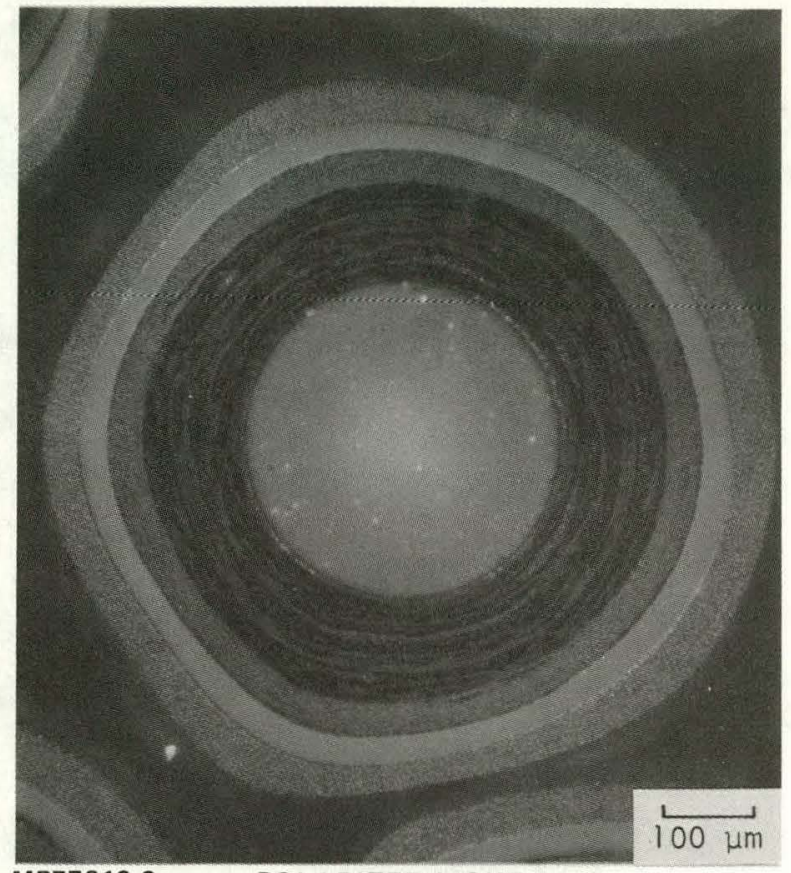

POLARIZED LIGHT

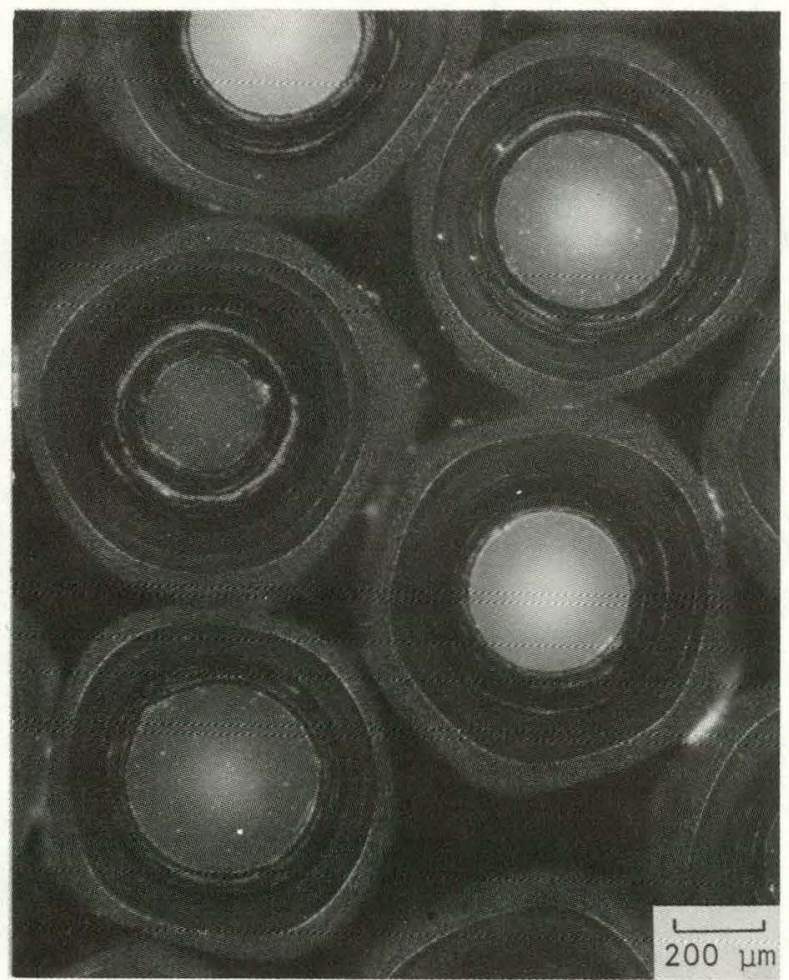

MP77012-2

POLARIZED LIGHT

Fig. 3-19. Representative photomicrographs of TRISO-coated $\mathrm{ThO}_{2}$ batch 6252-17-010 for capsule HT-34: bright field and polarized light 

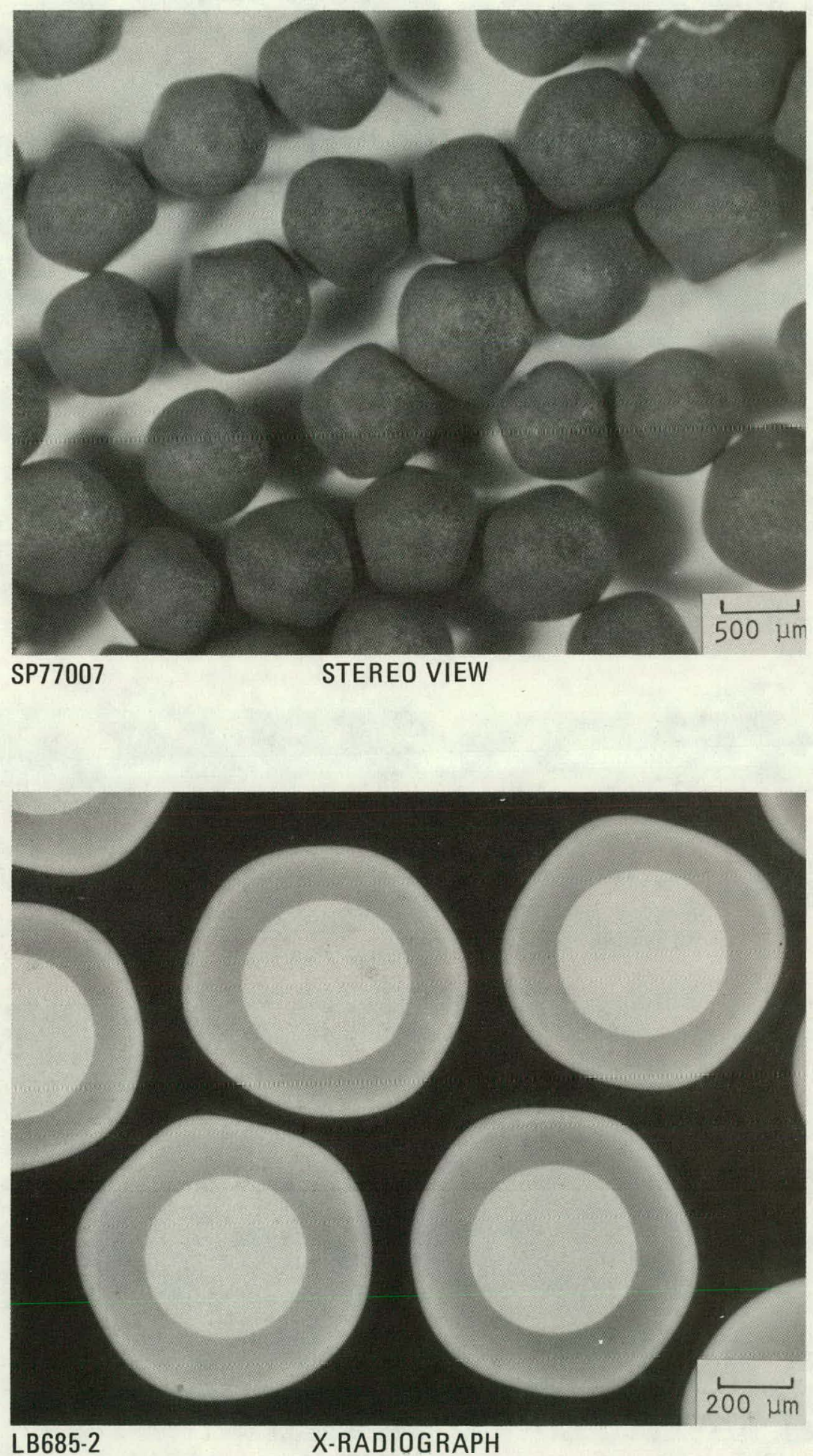

Fig. 3-20. Representative photomicrographs of TRISO-coated $\mathrm{ThO}_{2}$ batch 6252-20-010 for capsule HT-34: stereo view and X-radiograph 


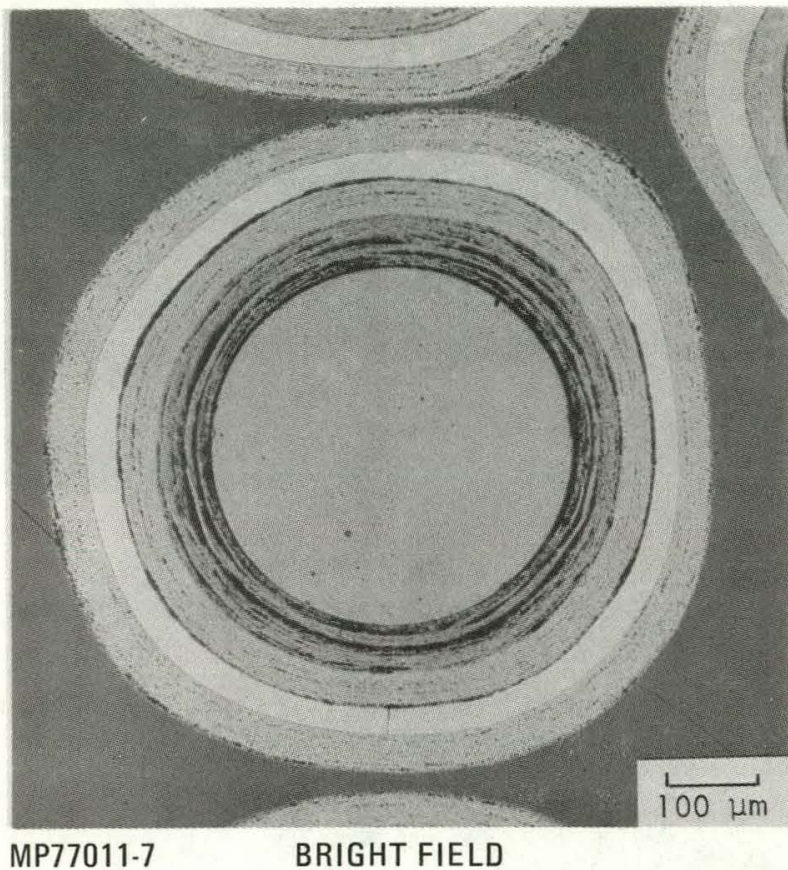

MP77011-7

BRIGHT FIELD

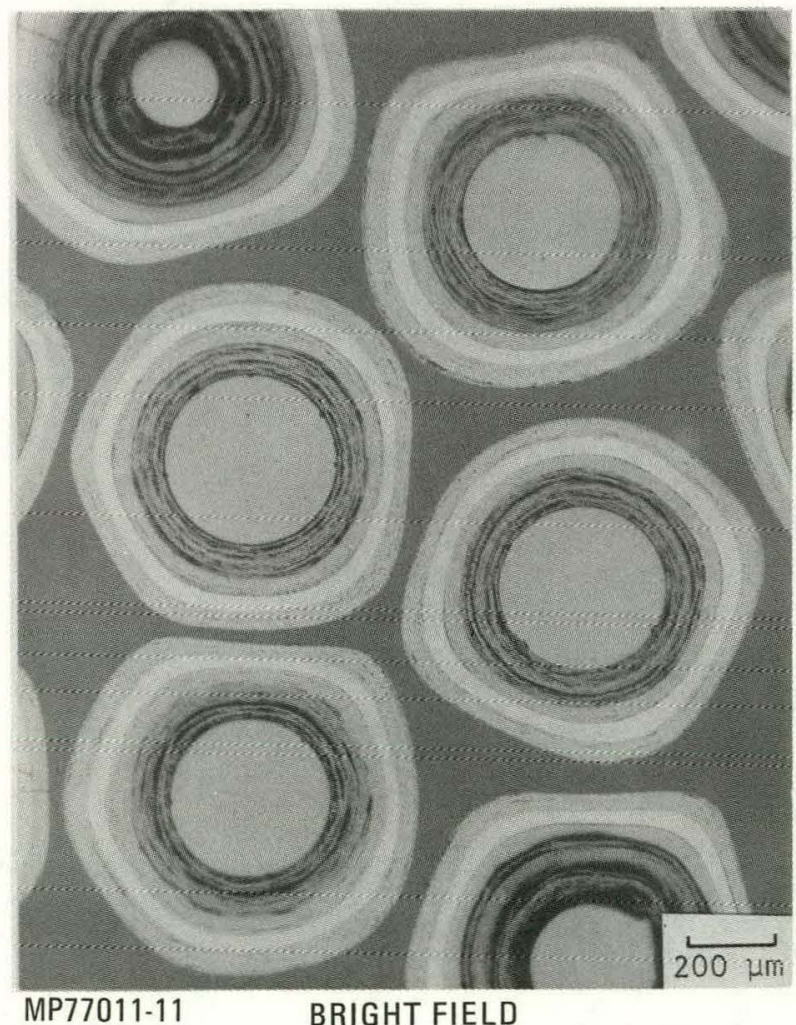

MP77011-11
BRIGHT FIELD

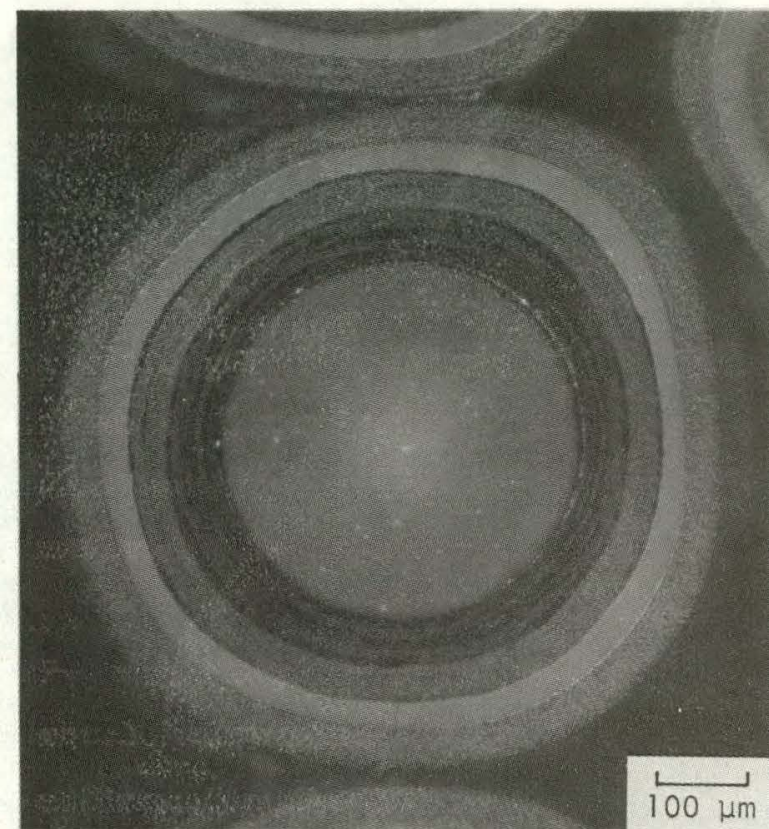

MP77011-8 POLARIZED LIGHT

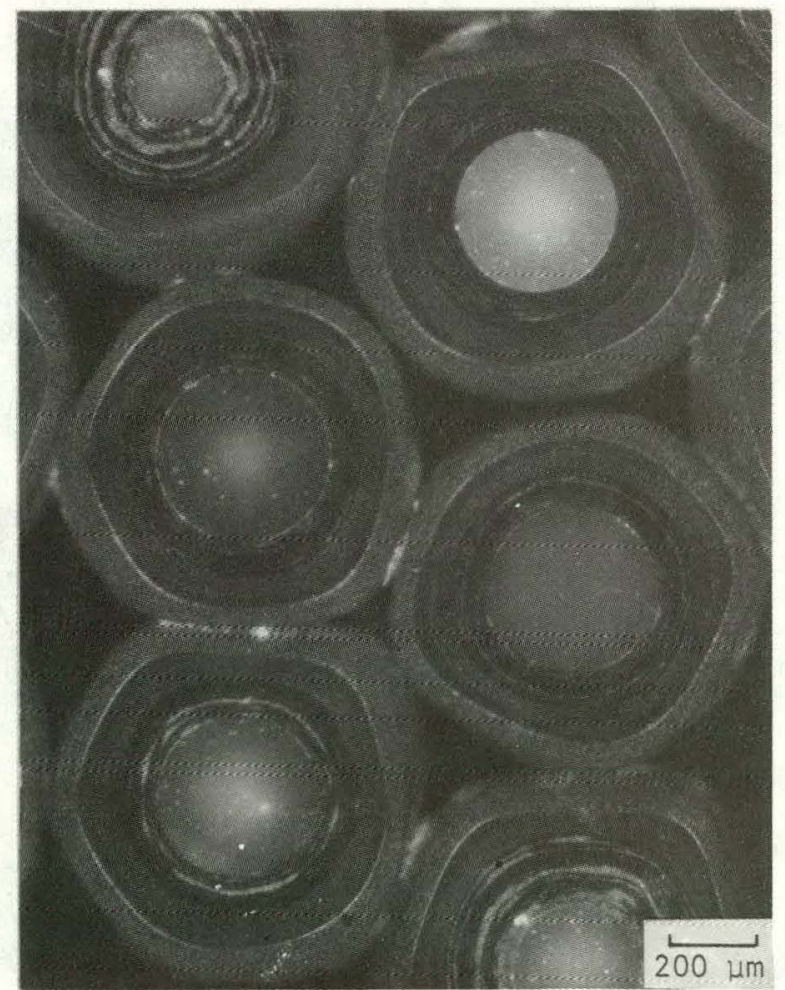

MP77011-2

POLARIZED LIGHT

Fig. 3-21. Representative photomicrographs of TRISO-coated $\mathrm{ThO}_{2}$ batch 6252-20-010 for capsule HT-34: bright field and polarized light 
TABLE 3-1

PRIMARY DESIGN VARIABLIS OF TRISO ThO 2 PARENT PARTICLE BATCHES FOR CAPSULE HT-34

\begin{tabular}{|c|c|c|c|c|c|c|c|}
\hline \multirow[b]{3}{*}{$\begin{array}{l}\text { Parent } \\
\text { Batch No. (a) }\end{array}$} & \multirow{3}{*}{$\begin{array}{l}\text { Buffer } \\
\text { Thicisness } \\
(\mu \mathrm{m})\end{array}$} & \multirow{3}{*}{$\begin{array}{c}\text { SiC } \\
\text { Flaws } \\
(\%)\end{array}$} & \multicolumn{4}{|c|}{ OPyC } & \multirow{3}{*}{$\begin{array}{c}\text { Coating } \\
\text { Diluent } \\
\text { Gas }\end{array}$} \\
\hline & & & \multicolumn{2}{|c|}{$\begin{array}{l}\text { Density } \\
\left(\mathrm{Mg} / \mathrm{m}^{3}\right)\end{array}$} & \multirow{2}{*}{$\begin{array}{l}\text { Micro- } \\
\text { porosity } \\
(\mu \ell / \mathrm{kg})\end{array}$} & \multirow{2}{*}{$\begin{array}{l}\text { Coating } \\
\text { Rate } \\
\text { ( } \mu \mathrm{m} / \mathrm{min})\end{array}$} & \\
\hline & & & $\begin{array}{l}\text { Liquid } \\
\text { Gradient (b) }\end{array}$ & Bulk & & & \\
\hline $6252-13-010$ & 60 & 10 & 1.80 & 1.60 & 60 & $\cdot 8$ & $\mathrm{H}_{2}$ \\
\hline $6252-14-010$ & 60 & 10 & 1.95 & 1.75 & 60 & 8 & $\mathrm{H}_{2}$ \\
\hline $6252-14-020$ & 610 & 30 & 1.95 & 1.75 & 60 & 8 & $\mathrm{H}_{2}$ \\
\hline $6252-15-010$ & 60. & 10 & 1.80 & 1.65 & 25 & 5 & $\mathrm{H}_{2}$ \\
\hline $6252-16-010$ & 61) & 10 & 1.95 & 1.80 & 25 & 5 & $\mathrm{H}_{2}$ \\
\hline $6252-20-010$ & 85 & 6 & 1.95 & 1.75 & 60 & 8 & $\mathrm{H}_{2}$ \\
\hline $6252-17-010$ & $8 \overline{5}$ & 6 & 1.95 & 1.80 & 25 & 5 & $\mathrm{H}_{2}$ \\
\hline $6252-07-020$ (c) & 61) & 38 & 1.90 & 1.75 & 50 & 6 & $\mathrm{Ar}$ \\
\hline
\end{tabular}

(a) The first eight numbers of the parent batch and the corresponding capsule samples are the same, i.e., batch 6252-13-010 is the parent batch of capsule sample 6252-13-0171-001.
(b) Specification density for the OPyC coating.
(c) Batch previously irradiated in capsules HT-31 and HT-33. 
TABLE 3-2

VARIABLES TO BE TESTED IN CAPSULE HT-34

\begin{tabular}{l|c}
\hline \multicolumn{1}{c|}{ Variable } & Capsule Samples (a) \\
\hline $1200^{\circ} \mathrm{C}$ Magazine & \\
OPyC properties & $8,10,11,13$ \\
Pressure vessel failure & $5,7,10$ \\
SiC flaw effect & 5,7 \\
Size effect on OPyC & 2,10 \\
OPyC-coating diluent gas. & $\mathrm{Al1}$ \\
Standard & 4 \\
$1500^{\circ} \mathrm{C}$ Magazine & $\mathrm{A} 11$ \\
Pressure vessel failure & $\mathrm{A} 11$ \\
Chemical behavior & $21,23,24,26$ \\
OPyC properties & $18,20,21$ \\
SiC flaw effect & 17,26 \\
Size effect on OPyC & 17 \\
Standard &
\end{tabular}

(a) Numbers given are the sample positions. 
TABLE 3-3

LOCATION AND DESCEIPTION OF PARTICLES IN END PLUGS OF CAPSULE HT-34

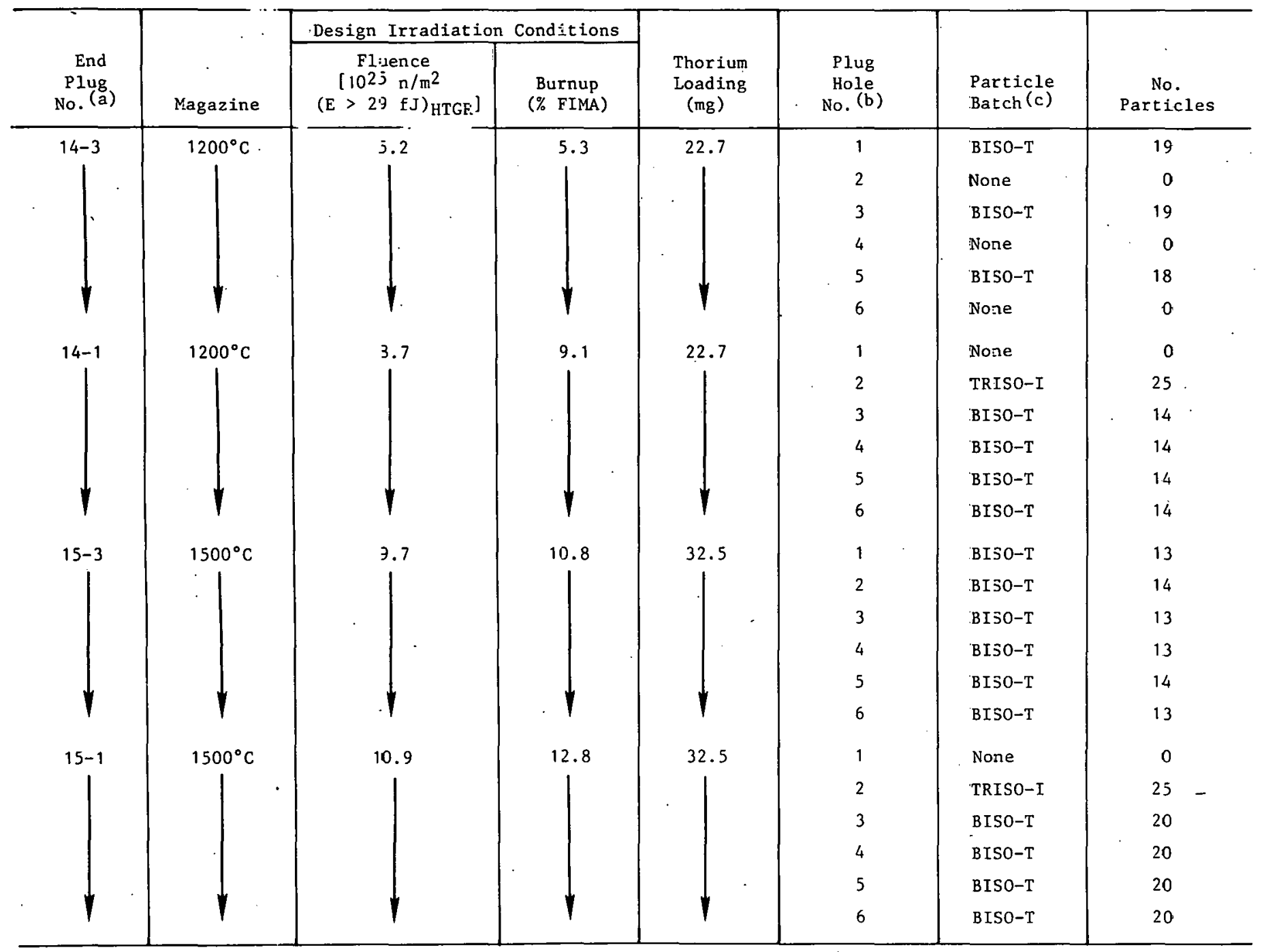

(a) See Fig. 2-1 for location of end plugs.

(b) Eole No. 1 has an index mark; other holes are numbered consecutively, clockwise from the top view.

(c) EISO-T = BISO $\mathrm{ThO}_{2}$ batch 7032-149 TRISO-I = TRISO inert batch 6351-040-0100, burned back. 
TABLE $3-4$

FABRICATION CONDITIONS OF TRISO ThO 2 PARENT BATCHES FOR CAPSULE HT-34 (a)

\begin{tabular}{|c|c|c|c|c|c|c|c|c|c|c|c|c|c|c|c|c|c|c|c|c|}
\hline \multirow{3}{*}{$\begin{array}{c}\text { Parent } \\
\text { Batch } \\
\text { No. (b) } \\
\end{array}$} & \multicolumn{3}{|c|}{ Buffer } & \multicolumn{5}{|c|}{ IPyC } & \multicolumn{5}{|c|}{ sic } & \multicolumn{7}{|c|}{ OPyC } \\
\hline & \multirow{2}{*}{$\begin{array}{c}\text { Coater } \\
\text { Charge } \\
(\mathrm{kG})\end{array}$} & \multirow{2}{*}{$\begin{array}{c}\text { Coating } \\
\text { Rate } \\
(\mu \mathrm{m} / \mathrm{min}) \\
\end{array}$} & \multirow{2}{*}{$\begin{array}{c}\text { Deposition } \\
\text { Gas/Diluent } \\
\text { and } \\
\text { Levitating Cas }\end{array}$} & \multirow{2}{*}{$\begin{array}{c}\text { Coatting } \\
\text { Rate } \\
(\mu \mathrm{m} / \mathrm{min})\end{array}$} & \multirow[b]{2}{*}{$\begin{array}{c}\text { Deposition } \\
\text { Gas }\end{array}$} & \multirow{2}{*}{$\begin{array}{c}\text { Diluent } \\
\text { Gas/ } \\
\text { Levitating } \\
\text { Gas }\end{array}$} & \multicolumn{2}{|c|}{$\begin{array}{l}\text { Deposition } \\
\text { Temperature } \\
\end{array}$} & \multirow{2}{*}{ 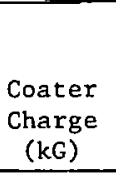 } & \multirow{2}{*}{$\begin{array}{c}\text { Coating } \\
\text { Rate } \\
(\mathrm{mam} / \mathrm{min}) \\
\end{array}$} & \multirow{2}{*}{$\begin{array}{c}\text { Deposition } \\
\text { Gas/Diluent } \\
\text { and } \\
\text { Levitating Gas }\end{array}$} & \multicolumn{2}{|c|}{ Deposition Temperalure } & \multirow{2}{*}{$\begin{array}{l}\text { Deposition } \\
\text { Control } \\
\text { Temperature } \\
\text { of Bed } \\
\text { ( Be) } \\
\left({ }^{\circ} \mathrm{C}\right)\end{array}$} & \multirow{2}{*}{$\begin{array}{c}\text { Coater } \\
\text { Charge } \\
(\mathrm{kG}) \\
\end{array}$} & \multirow{2}{*}{$\begin{array}{c}\text { Coating } \\
\text { Rate } \\
(\mu \mathrm{m} / \mathrm{min}) \\
\end{array}$} & \multirow[b]{2}{*}{$\begin{array}{c}\text { Deposition } \\
\text { Gas }\end{array}$} & \multirow{2}{*}{$\begin{array}{c}\text { Diluent } \\
\text { Gas } / \\
\text { Levitating } \\
\text { Gas }\end{array}$} & \multicolumn{2}{|c|}{$\begin{array}{l}\text { Deposition } \\
\text { Temperature }\end{array}$} \\
\hline & & & & & & & $\begin{array}{c}\text { Bed } \\
\left({ }^{\circ} \mathrm{C}\right) \\
\end{array}$ & $\begin{array}{c}\text { Control } \\
\left({ }^{\circ} \mathrm{C}\right)\end{array}$ & & & & $\begin{array}{r}\text { Bed } \\
\left({ }^{\circ} \mathrm{C}\right) \\
\end{array}$ & $\begin{array}{l}\text { Control } \\
\left({ }^{\circ} \mathrm{C}\right)\end{array}$ & & & & & & $\begin{array}{l}\text { Bed } \\
\left({ }^{\circ} \mathrm{C}\right) \\
\end{array}$ & $\begin{array}{c}\text { Control } \\
\left({ }^{\circ} \mathrm{C}\right)\end{array}$ \\
\hline $6252-07-025$ & 15 & 11.0 & $\mathrm{C}_{2} \mathrm{H}_{2} / \mathrm{Ar}$ & 4.4 & $\mathrm{C}_{2} \mathrm{H}_{2} / \mathrm{C}_{3} \mathrm{H}_{6}$ & $\mathrm{Ar} / \mathrm{Ar}$ & $\mathrm{ND}(\mathrm{c})$ & 1300 & 19.5 & 0.16 & $\mathrm{CH}_{3} \mathrm{SiCl}_{3} / \mathrm{H}_{2}{ }^{(\mathrm{d})}$ & ND & 1625 & 1625 & 11.0 & 5.3 & $\mathrm{C}_{2} \mathrm{H}_{2} / \mathrm{C}_{3} \mathrm{H}_{6}$ & $\mathrm{Ar} / \mathrm{Ar}$ & ND & 1540 \\
\hline 6252-13-010 & 17 & 9.0 & $\mathrm{C}_{2} \mathrm{H}_{2} / \mathrm{Ar}$ & 4.1 & $\mathrm{C}_{2} \mathrm{H}_{2} / \mathrm{C}_{3} \mathrm{H}_{6}$ & $\mathrm{H}_{2} / \mathrm{Ar}$ & 1185 & 1350 & 10.5 & 0.32 & $\mathrm{CH}_{3} \mathrm{SiCl}_{3} / \mathrm{H}_{2}$ & ND & 1650 & 1650 & 13.5 & 8.6 & $\mathrm{C}_{2} \mathrm{H}_{2} / \mathrm{C}_{3} \mathrm{H}_{6}$ & $\mathrm{H}_{2} / \mathrm{Ar}$ & 1400 & 1420 \\
\hline $6252-14-010$ & 17 & 10.0 & $\mathrm{C}_{2} \mathrm{H}_{2} / \mathrm{Ar}$ & 4.0 & $\mathrm{C}_{2} \mathrm{H}_{2} / \mathrm{C}_{3} \mathrm{H}_{6}$ & $\mathrm{H}_{2} / \mathrm{Ar}$ & ND & 1360 & 11.0 & 0.31 & $\mathrm{CH}_{3} \mathrm{SiCl}_{3} / \mathrm{H}_{2}$ & ND & 1650 & 1650 & 13.5 & 8.3 & $\mathrm{C}_{2} \mathrm{H}_{2} / \mathrm{C}_{3} \mathrm{H}_{6}$ & $\mathrm{H}_{2} / \mathrm{Ar}$ & 1295 & 1315 \\
\hline $6252-14-020$ & 17 & 9.0 & $\mathrm{C}_{2} \mathrm{H}_{2} / \mathrm{Ar}$ & 4.7 & $\mathrm{C}_{2} \mathrm{H}_{2} / \mathrm{C}_{3} \mathrm{H}_{6}$ & $\mathrm{H}_{2} / \mathrm{Ar}$ & ND & 1350 & 20.5 & 0.28 & $\mathrm{CH}_{3} \mathrm{SiCl}_{3} / \mathrm{H}_{2}$ & ND & 1650 & 1650 & 13.0 & 8.0 & $\mathrm{C}_{2} \mathrm{H}_{2} / \mathrm{C}_{3} \mathrm{H}_{6}$ & $\mathrm{H}_{2} / \mathrm{Ar}$ & 1275 & 1315 \\
\hline $6252-15-010$ & 17 & 10.2 & $\mathrm{C}_{2} \mathrm{H}_{2} / \mathrm{Ar}$ & 4.2 & $\mathrm{C}_{2} \mathrm{H}_{2} / \mathrm{C}_{3} \mathrm{H}_{6}$ & $\mathrm{H}_{2} / \mathrm{Ar}$ & ND & 1360 & 10.5 & 0.32 & $\mathrm{CH}_{3} \mathrm{SiCl}_{3} / \mathrm{H}_{2}$ & ND & 1650 & 1650 & 13.5 & 5.3 & $\mathrm{C}_{2} \mathrm{H}_{2} / \mathrm{C}_{3} \mathrm{H}_{6}$ & $\mathrm{H}_{2} / \mathrm{Ar}$ & 1385 & 1405 \\
\hline $6252-16-010$ & 17 & 10.2 & $\mathrm{C}_{2} \mathrm{H}_{2} / \mathrm{Ar}$ & 4.2 & $\mathrm{C}_{2} \mathrm{H}_{2} / \mathrm{C}_{3} \mathrm{H}_{6}$ & $\mathrm{H}_{2} / \mathrm{Ar}$ & ND & 1360 & 10.5 & 0.33 & $\mathrm{CH}_{3} \mathrm{SiCl}_{3} / \mathrm{H}_{2}$ & ND & 1650 & 1650 & 13.5 & 6.0 & $\mathrm{C}_{2} \mathrm{H}_{2} / \mathrm{C}_{3} \mathrm{H}_{6}$ & $\mathrm{H}_{2} / \mathrm{Ar}$ & 1280 & 1310 \\
\hline $6252-17-010$ & 17 & 13.0 & $\mathrm{C}_{2} \mathrm{H}_{2} / \mathrm{Ar}$ & 4.2 & $\mathrm{C}_{2} \mathrm{H}_{2} / \mathrm{C}_{3} \mathrm{H}_{6}$ & $\mathrm{H}_{2} / \mathrm{Ar}$ & 1195 & 1330 & 22.5 & 0.23 & $\mathrm{CH}_{3} \mathrm{SiCl}_{3} / \mathrm{H}_{2}$ & ND & 1650 & 1650 & 14.0 & 5.4 & $\mathrm{C}_{2} \mathrm{H}_{2} / \mathrm{C}_{3} \mathrm{H}_{6}$ & $\mathrm{H}_{2} / \mathrm{Ar}$ & 1280 & 1310 \\
\hline $6252-20-010$ & 17 & 13.0 & $\mathrm{C}_{2} \mathrm{H}_{2} / \mathrm{Ar}$ & 4.2 & $\mathrm{c}_{2} \mathrm{H}_{2} / \mathrm{C}_{3} \mathrm{H}_{6}$ & $\mathrm{H}_{2} / \mathrm{Ar}$ & 1195 & 1330 & 22.5 & 0.24 & $\mathrm{Ch}_{3} \mathrm{SiCl}_{3} / \mathrm{H}_{2}$ & ND & 1650 & 1650 & 14.0 & 9.0 & $\mathrm{C}_{2} \mathrm{H}_{2} / \mathrm{C}_{3} \mathrm{H}_{6}$ & $\mathrm{H}_{2} / \mathrm{Ar}$ & 1275 & 1315 \\
\hline
\end{tabular}

(a) Coated in 240 -mm-i.d. dry coater with a cone gas distributor containing an extension nozzle through center of core. Deposition and diluent gases premixed and enter near top of extensinn nnzz.le; levitating

gas enters at bottom of cone.
(b) The first eight numbers of the parent batches and the corresponding capsule samples are the same, 1.e., batch 6252-13-010 is the parent batch of capsule sample 6252-13-0171-001.

(c) $)_{\mathrm{ND}}=$ not firsternt numbers

(d) Levitating gas was $\mathrm{H}_{2}$ and argon mixture. 


\section{PAGES 3-37 to 3-38 WERE INTENTIONALLY LEFT BLANK}


TABLE $3-5$

NUMERICAL CROSS REFERENCE OF HT-34 CAPSULE SPECIMENS

\begin{tabular}{|c|c|c|c|c|c|}
\hline \multirow{2}{*}{$\begin{array}{l}\text { Parent Batch } \\
\text { Data Retrieval } \\
\text { No. }\end{array}$} & \multicolumn{2}{|c|}{ After Density Separation } & \multicolumn{2}{|c|}{ After Heat Treatment } & \multirow{2}{*}{$\begin{array}{c}\text { Capsule Specimen } \\
\text { Data Retrieval } \\
\text { No. }\end{array}$} \\
\hline & $\begin{array}{c}\text { GA } \\
\text { Notebook No. }\end{array}$ & $\begin{array}{c}\text { Data Retrieval } \\
\text { No. }\end{array}$ & $\begin{array}{c}\text { GA Notebook } \\
\text { No. }\end{array}$ & $\begin{array}{c}\text { Data Retrieval } \\
\text { No. }\end{array}$ & \\
\hline$; 6252-07-0200$ & $2995-114$ & $6252-07-0262$ & $6627-86-13$ & $6252-07-0262$ & $\left\{\begin{array}{l}6252-07-0262-001 \\
6252-07-0262-002\end{array}\right.$ \\
\hline $6252-13-0100$ & $6627-69-3$ & $6252-13-0110$ & $6627-86-7$ & $6252-13-0111$ & $6252-13-0161-001$ \\
\hline $6252-13-0100$ & $6627-72-1$ & $6252-13-0120$ & $6627-86-1$ & $6252-13-0171$ & $6252-13-0171-001$ \\
\hline $6252-14-0100$ & $6627-68-1$ & $6252-14-0130$ & $6627-86-2$ & $6252-14-0131$ & $6252-14-0181-001$ \\
\hline $6252-14-0100$ & $6627-68-2$ & $6252-14-0110$ & $6627-86-8$ & $6252-14-0111$ & $\left\{\begin{array}{l}6252-14-0161-001 \\
6252-14-0161-002\end{array}\right.$ \\
\hline $6252-1.4-0100$ & $662 T-68-3$ & $6252-14-0120$ & $6627-86-3$ & $6252-1 \leq-0121$ & $6252-14-0171-001$ \\
\hline $6252-14-0200$ & $6627-59-3$ & $6252-14-0210$ & $6627-86-6$ & $6252-14-0211$ & $6252-14-0261-001$ \\
\hline $6252-14-0200$ & $6627-73-1$ & $6252-14-0220$ & $6627-86-5$ & $6252-14-0221$ & $6252-14-0271-001$ \\
\hline $6252-15-0100$ & $6627-70-1$ & $6252-15-0120$ & $6627-86-4$ & $6252-15-0121$ & $6252-15-0171-001$ \\
\hline $6252-15-0100$ & $6627-70-3$ & $6252-15-0110$ & $6627-86-9$ & $6252-15-0111$ & $6252-15-0161-001$ \\
\hline $6252-16-010 \dot{0}$ & $6627-71-2$ & $6252-16-0110$ & $6627-86-10$ & $6252-16-0111$ & $6252-16-0161-001$ \\
\hline $6252-17-0100$ & $6627-56-2$ & $6252-17-0110$ & $6627-86-12$ & $6252-17-0111$ & $6252017-0161-001$ \\
\hline $6252-20-0100$ & $6627-52-2$ & $6252-20-0110$ & $662 T-86-11$ & $6252-20-0111$ & $\left\{\begin{array}{l}6252-20-0161-001 \\
6252-20-0161-002\end{array}\right.$ \\
\hline
\end{tabular}


TABLE 3-6

CALCULATION OF PARTICLE LOADINGS FOR CAPSULE HT-34

\begin{tabular}{|c|c|c|c|c|c|c|c|}
\hline Sample Batch No. & $\begin{array}{l}\text { Particle } \\
\text { Density } \\
\text { Fraction }\end{array}$ & Magazine (a) & $\begin{array}{c}\text { Density } \mathrm{ThO}_{2} \\
\left(\mathrm{Mg} / \mathrm{m}^{3}\right)\end{array}$ & $\begin{array}{l}\text { Kernel Diam } \\
\left(m \times 10^{-6}\right)\end{array}$ & $\begin{array}{l}\text { Kerne1 Vol } \\
\left(\mathrm{m}^{3} \times 10^{-11}\right)\end{array}$ & $\begin{array}{c}\mathrm{ThO}_{2} / \\
\text { Particle } \\
\left(\mathrm{g} \times 10^{-10}\right)\end{array}$ & $\begin{array}{c}\text { No. } \\
\text { Particles }\end{array}$ \\
\hline $6252-13-0171-001$ & Low & High & 9.78 & 442.1 & 4.52 & 4.42 & 85 \\
\hline $6252-14-0181-001$ & Low & High & 9.90 & 445.6 & 4.63. & 4.59 & 82 \\
\hline $6252-14-0171-001$ & High & Low & 9.90 & 451.9 & 4.83 & 4.78 & 56 \\
\hline $6252-15-0177-001$ & Low & High & 9.90 & 447.1 & 4.68 & 4.63 & 81 \\
\hline $6252-14-0271-001$ & Low & High & 96.841 & 445.1 & 4.62 & 4.54 & 83 \\
\hline $6252-14-0261-001$. & High & Low & 9.841 & 451.9 & 4.83 & 4.76 & 56 \\
\hline $6252-13-0161-001$ & Nominal & Low & 9.78 & 449.7 & 4.76 & 4.66 & 57 \\
\hline $6252-14-0161-001$ & Nominal & Low & 9.90 & 447.8 & 4.70 & 4.65 & 57 \\
\hline $6252-14-0161-002$ & Nominal & High & 9.90 & 447.8 & 4.70 & 4.65 & 81 \\
\hline $6252-15-0161-001$ & Nominal & Low & 9.90 & 451.0 & 4.80 & 4.75 & 56 \\
\hline $6252-16-0161-001$ & Nominal & Low & 9.90 & 451.9 & 4.83 & 4.78 & 56 \\
\hline $6252-20-0161-00 i$ & Noninal & Low & 9.78 & 451.5 & 4.82 & 4.71 & 56 \\
\hline $6252-20-0161-002$ & Nominal & High & 9.78 & 451.5 & 4.82 & 4.71 & 80 \\
\hline $6252-17-0161-001$ & Nominal & $\mathrm{High}$ & 9.78 & 450.0 & 4.77 & 4.66 & 81 \\
\hline $6252-07-0262-001$ & Nominal & Low & 9.91 & 447.9 & 4.70 & .4 .66 & 57 \\
\hline $6252-07-0262-002$ & Nominal & High & 9.91 & 447.9 & 4.70 & 4.66 & 81 \\
\hline
\end{tabular}

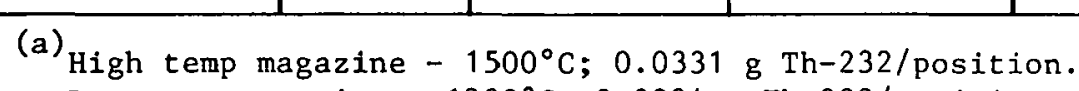

Low temp magazine $-1200^{\circ} \mathrm{C} ; 0.0234 \mathrm{~g}$ Th-232/position. 
TABLE 3-7

INPUT PARANETERS USED IN TRISO MONTE-NOCO CODE

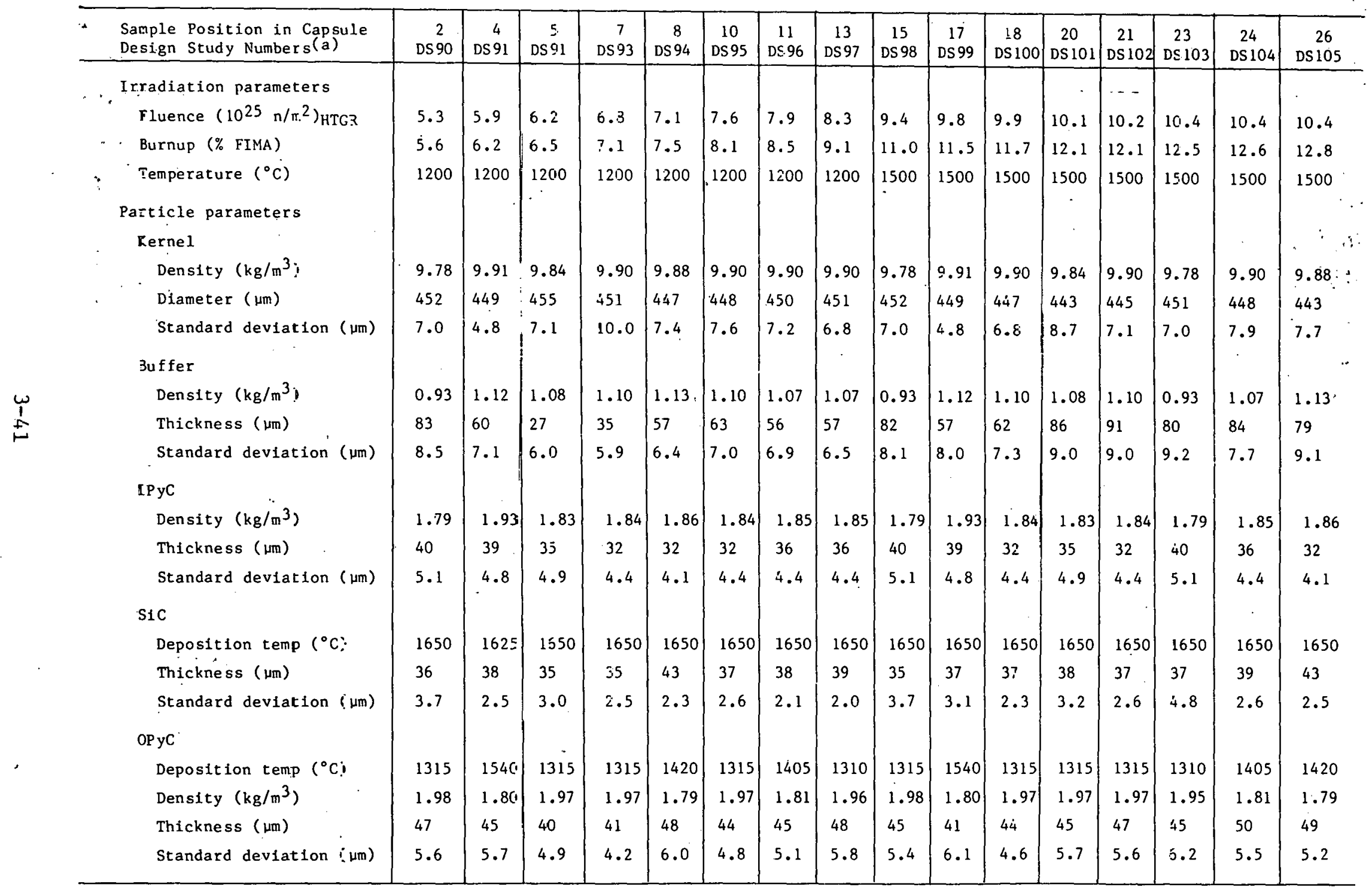

(a) General Atomic Company Notebook \#7440, unpublished data. 
TABLE 3-8

PREDICTED PARTICLE PERFORMANCE FOR CAPSULE HT- 34

\begin{tabular}{|c|c|c|c|c|c|c|c|c|c|}
\hline \multirow{3}{*}{$\begin{array}{c}\text { Particle Batch } \\
\text { Data Retrieval } \\
\text { Number }\end{array}$} & \multirow{3}{*}{$\begin{array}{l}\text { Sample } \\
\text { Posi- } \\
\text { tion }\end{array}$} & \multirow{3}{*}{$\begin{array}{l}\text { Design } \\
\text { Study } \\
\text { Number }\end{array}$} & \multicolumn{3}{|c|}{ Design Irradiation Conditions } & \multirow{2}{*}{\multicolumn{4}{|c|}{ Predicted Failure Fractions }} \\
\hline & & & & \multirow{2}{*}{$\mid \begin{array}{c} \\
\text { Burnup } \\
(\% \cdot \text { FIMA })\end{array}$} & \multirow{2}{*}{$\begin{array}{l}\text { Tempera- } \\
\text { ture } \\
\left({ }^{\circ} \mathrm{C}\right)\end{array}$} & & & & \\
\hline & & & $\left(10^{25} \mathrm{n} / \mathrm{m}^{2}\right)_{\mathrm{HFIR}}$ & & & OPy & Intact & $\mathrm{OP}$ & C Failed \\
\hline $6252-20-0161-001$ & 2 & DS90 & 5.3 & 5.6 & 1200 & $0^{(\mathrm{b})}$ & $(0-0)^{(c)}$ & $0^{(b)}$ & $(1.0-0)^{(c)}$ \\
\hline$-07-0262-001$ & 4 & DS91 & 5.9 & 6.2 & 1200 & 0 & $(.18-0)$ & 0.87 & $(1.0-0)$ \\
\hline$-14-0261-001$ & 5 & DS92 & 6.2 & 6.5 & 1200 & 0.59 & $(.98-.13)$ & 1.0 & $(1.0-.86)$ \\
\hline$-14-0171-001$ & 7 & DS93 & 6.8 & 7.1 & 1200 & 0.51 & $(.99-.01)$ & 1.0 & $(1.0-.85)$ \\
\hline$-13-0161-001$ & 8 & DS94 & 7.1 & 7.5 & 1200 & 0 & $(.77-0)$ & 1.0 & $(1.0-0)$ \\
\hline$-14-0161-001$ & 10 & DS95 & 7.6 & 8.1 & 1200 & 0 & $(.78-0)$ & 1.0 & $(1.0-.02)$ \\
\hline$-15-0161-001$ & 11 & DS 96 & 7.9 & 8.5 & 1200 & 0 & $(.84-0)$ & 1.0 & $(1.0-.05)$ \\
\hline$-16-0161-001$ & 13 & DS 97 & 8.3 & 9.1 & 1200 & 0 & $(.91-0)$ & 1.0 & $(1.0-.13)$ \\
\hline$-20-0161-002$ & 15 & DS98 & 9.4 & 11.0 & 1500 & 0 & $(.80-0)$ & 1.0 & $(1.0-.07)$ \\
\hline$-07-0262-002$ & 17 & DS99 & 9.8 & 11.5 & 1500 & 0.94 & $(1.0-.31)$ & 1.0 & $(1,0-1,0)$ \\
\hline$-14-0161-002$ & 18 & DS100 & 9.9 & 11.7 & 1500 & 0.90 & $(1.0-.11)$ & 1.0 & $(1.0-.99)$ \\
\hline$-14-0271-001$ & 20 & DS 101 & 10.1 & 12.1 & 1500 & 0.11 & $(1.0-0)$ & 1.0 & $(1.0-.57)$ \\
\hline$-14-0181-001$ & 21 & DS 102 & 10.2 & 12.2 & 1500 & 0.12 & $(1.0-0)$ & 1.0 & $(1.0-.70)$ \\
\hline$-17-0161-001$ & 23 & DS 103 & 10.4 & 12.5 & 1500 & 0.09 & $(.97-0)$ & 1.0 & $(1.0-.43)$ \\
\hline$-15-0171-001$ & 24 & DS104 & 10.4 & 12.6 & 1500 & 0.04 & $(.99-0)$ & 1.0 & $(1.0-.70)$ \\
\hline$-13-0171-001$ & 26 & DS 105 & 10.4 & 12.8 & 1500 & 0.36 & $(1.0-.01)$ & 1.0 & $(1.0-.93)$ \\
\hline
\end{tabular}

(a) Referenced from GA internal document.

(b) Predicted failure fraction based on a mean Apparent Failure Stress of $-0.34 \times 10^{4}$ psi.

(c) Range of predicted failure fractions based on an Apparent Failure Stress range (two tailed test at $90 \%$ confidence level) of $-2.03 \times 10^{4}$ to $1.35 \times 10^{4}$ psi. 
TABLE 3-9

FERNEL PROPERTIES OF TRISO ThO 2 PARENT BATCHES FOR CAPSULE HT-34

\begin{tabular}{|c|c|c|c|c|c|c|c|c|}
\hline \multirow[b]{2}{*}{$\begin{array}{l}\text { Particle } \\
\text { Batch Vo. }\end{array}$} & \multicolumn{2}{|c|}{$\begin{array}{c}\text { Kernel Batch } \\
\text { No. }\end{array}$} & \multicolumn{2}{|c|}{$\begin{array}{c}\text { Diameter } \\
(\mu \mathrm{a})\end{array}$} & \multirow[b]{2}{*}{$\begin{array}{c}\text { Density }(\mathrm{b}) \\
\left(\mathrm{Mg} / \mathrm{m}^{3}\right)\end{array}$} & \multicolumn{3}{|c|}{$\begin{array}{c}\text { Impurities } \\
(\mathrm{ppm}-\mathrm{wt})\end{array}$} \\
\hline & $\begin{array}{c}\text { Data } \\
\text { Retrieval No. }\end{array}$ & Manufacturer & Mean & $\mathrm{SD}$ & & $\mathrm{Fe}$ & $\mathrm{Cr}$ & $\mathrm{Ni}$ \\
\hline $6252-07-025$ & $4222-08-0130$ & FKB-1075-1-3 & 453 & 8.3 & 9.91 & 28 & 52 & 17 \\
\hline $6252-13-010$ & $4222-09-0300$ & $88-1-118-2-2 / 3$ & .454 & 8.3 & 9.88 & 64 & 16 & 42 \\
\hline $6252-14-010$ & $4222-09-0400$ & $90-1-120$ & 451 & 9.1 & 9.90 & 55 & 16 & 40 \\
\hline $6252-14-020$ & $4222-09-0100$ & $88-1-118-2$ & 451 & 8.1 & 9.84 & 61 & 35 & 76 \\
\hline $6252-15-010$ & $4222-09-0400$ & $90-1-120$ & 456 & 8.4 & 9.90 & 55 & 16 & 40 \\
\hline $6252-16-010$ & $4222-09-0400$ & $90-1-120$ & 455 & 8.4 & 9.90 & 55 & 16 & 40 \\
\hline $6252-17-010$ & $4222-09-0200$ & $88-1-118-3$ & 455 & 8.9 & 9.78 & 53 & 13 & 40 \\
\hline $6252-20-010$ & $4222-09-0200$ & $88-1-118-3$ & 456 & 8.6 & 9.78 & 53 & 13 & 40 \\
\hline
\end{tabular}

(a) Measured by X-radiography.

(b) Measured by mercury porosimetry. 
THIS PAGE

\section{WAS INTENTIONALLY LEFT BLANK}


TABLE $3-10$

COATING PROPERTIES OF TRISO ThO 2 PARENT BATCHES FOR CAPSULE HT- 34

\begin{tabular}{|c|c|c|c|c|c|c|c|c|c|c|c|c|c|c|c|c|c|c|c|c|c|c|c|c|}
\hline \multirow{4}{*}{$\begin{array}{c}\text { Parent } \\
\text { Batch No. }\end{array}$} & \multirow{2}{*}{\multicolumn{3}{|c|}{ Buffer }} & \multicolumn{6}{|c|}{ IPyC } & \multicolumn{5}{|c|}{ sic } & \multicolumn{10}{|c|}{ OPyc } \\
\hline & & & & \multirow{2}{*}{\multicolumn{2}{|c|}{$\begin{array}{c}\text { Thickness }(b, c) \\
(\mu \mathrm{m})\end{array}$}} & \multirow{2}{*}{\multicolumn{2}{|c|}{$\begin{array}{l}\text { Liquid Gradient } \\
\text { Density } \\
\left(\mathrm{Mg} / \mathrm{m}^{3}\right)\end{array}$}} & \multirow{2}{*}{\multicolumn{2}{|c|}{$\mathrm{BAF}_{\mathrm{O}}(\mathrm{d})$}} & \multirow{2}{*}{\multicolumn{2}{|c|}{$\begin{array}{c}\text { Thickne.ss. } \\
(\mu \mathrm{m})\end{array}$}} & \multirow{2}{*}{\multicolumn{2}{|c|}{$\begin{array}{l}\text { Liquid Gradient } \\
\text { Density } \\
\left(\mathrm{Mg} / \mathrm{m}^{3}\right)\end{array}$}} & \multirow{3}{*}{$\begin{array}{l}\text { Flaw (e) } \\
\text { Frequency } \\
(\%)\end{array}$} & \multirow{2}{*}{\multicolumn{2}{|c|}{$\begin{array}{c}\text { Thicknesss (b) } \\
(\mu \mathrm{m})\end{array}$}} & \multicolumn{3}{|c|}{$\begin{array}{l}\text { Density } \\
\left(\mathrm{mg} / \mathrm{m}^{3}\right)\end{array}$} & \multirow{3}{*}{$\begin{array}{c}\begin{array}{c}\text { Accessible } \\
\text { porosity }(\mathrm{f}) \\
(\mathrm{m} 1 / \mathrm{kg})\end{array} \\
\end{array}$} & \multirow{3}{*}{$\begin{array}{c}\text { Oriented } \\
\text { Porosity } \\
(\%)\end{array}$} & \multirow[b]{3}{*}{ Faceting $(\mathrm{g})$} & \multirow{2}{*}{\multicolumn{2}{|c|}{$\mathrm{BAF}_{\mathrm{o}}(\mathrm{d})$}} \\
\hline & \multicolumn{2}{|c|}{$\begin{array}{c}\text { Thickness }(a, b) \\
(\mu \mathrm{m})\end{array}$} & \multirow{2}{*}{$\begin{array}{l}\text { Dens1 } 1 \mathrm{ty} \\
\left(\mathrm{Mg} / \mathrm{m}^{3}\right)\end{array}$} & & & & & & & & & & & & & & Liquid & Gradient & & & & & & \\
\hline & Mean & SD & & Mean & SD & Mean & SD & Mean & SD & Mean & SD & Mean & SD & & Mean & SD & Mean & SD & Bulk & & & & Mean & SD \\
\hline $6252-07-025$ & 63 & 11.4 & 1.12 & 39 & 4.8 & 1.93 & 0.011 & 1.10 & 0.011 & 38 & 3.3 & 3.21 & 0.004 & 38 & 48 & 6.2 & 1.80 & 0.025 & 1.65 & 48 & 0 & ${ }_{N D}^{(h)}$ & 1.027 & 0.0030 \\
\hline $6252-13-010$ & 55 & 9.7 & 1.13 & 32 & 4.1 & 1.86 & 0.032 & 1.06 & 0.007 & 42 & 2.3 & 3.22 & 0.002 & 16 & 50 & 4.9 & 1.79 & 0.006 & 1.61 & 57 & 0 & 6.09 & 1.031 & 0.0027 \\
\hline $6252-14-010$ & 62 & 12.0 & 1.10 & 32 & 4.4 & 1.84 & 0.028 & 1.06 & 0.006 & 37 & 2.5 & 3.22 & $<0.001$ & 9 & 48 & 5.2 & 1.97 & 0.005 & 1.76 & 57 & 0 & 7.09 & 1.041 & 0.0042 \\
\hline $6252-14-020$ & 55 & 10.7 & 1.08 & 35 & 4.9 & 1.83 & 0.014 & 1.06 & 0.006 & 36 & 2.7 & 3.21 & $<0.001$ & 30 & 45 & 6.3 & 1.97 & 0.004 & 1.79 & 54 & 0 & 7.91 & 1.040 & 0.0048 \\
\hline $6252-15-010$ & 54 & 11.4 & 1.07 & 36 & 4.4 & 1.85 & 0.020 & 1.06 & 0.006 & 38 & 2.4 . & 3.22 & 0.003 & 12 & 48 & 6.3 & 1.81 & 0.011 & 1.68 & 21 & 0 & 8.76 & 1.033 & 0.0032 \\
\hline $6252-16-010$ & 58 & 12.3 & 1.07 & 36 & 4.4 & 1.85 & 0.020 & 1.06 & 0.007 & 39 & 2.0 & 3.21 & 0.005 & 12 & 50 & 5.7 & 1.96 & 0.003 & 1.82 & 25 & 0 & 8.31 & 1.048 & 0.0048 \\
\hline $6252-17-010$ & 90 & 22.3 & 0.93 & 40 & 5.1 & 1.79 & 0.014 & 1.05 & 0.008 & 36 & 3.4 & 3.22 & 0.005 & 6 & 49 & 7.1 & 1.95 & 0.004 & 1.80 & 28 & 0 & 9.69 & 1.049 & 0.0043 \\
\hline $6252-20-010$ & 86 & 19.2 & 0.93 & 40 & 5.1 & 1.79 & 0.014 & 1.05 & 0.006 & 37 & 3.4 & 3.21 & 0.003 & 6 & 48 & 5.8 & 1.98 & 0.005 & 1.74 & 59 & 0.02 & 8.58 & 1.041 & 0.0046 \\
\hline
\end{tabular}

(a) Calculated (after SiC coating deposited) by subtracting IPyC thickness from measured buffer and IPyC thickness.

(a) Calculated (after SiC coating deposited) by subtractin
(b) Measured by contact X-radiography on $\geq 200$ particles.

(c) Measured at BISO stage.

(d) Measured with the Seibersdorf unit at GA using a $24-\mu m$ spot on 50 particles.

(e) Counted by a visual examination of gold spors in the sic coatings, \% ur particles will flaws.

(A) The amount of mercury which penetrates the coatings from 250 to $10,000 \mathrm{psi}$.

(g) Measured using 1ine-intercept technique on 100 particles on $\mathrm{X}$-radiograph plates.

Equarion used

$$
100 x \frac{\sqrt{2}}{n} \sum_{i=1}^{n}\left(\frac{x_{1 i}-x_{2 i}}{x_{1 i}+x_{2 i}}\right) \quad \begin{aligned}
\text { where, } & n=\text { number of particles } \\
x_{1} & =\text { OPyC thickness on one side of particle } \\
x_{2} & =O P y C \text { thickness on other side of particle }
\end{aligned}
$$

$(h)_{\mathrm{ND}}=$ not determined. 
THIS PAGE

\section{WAS INTENTIONALLY LEFT BLANK}


TABLE $3-11$
PROPERTIES OF TOTAL COATED PARTICLES OF TRISO ThO ${ }_{2}$ PARENT BATCHES FOR CAPSULE HT- 34

\begin{tabular}{|c|c|c|c|c|c|c|c|c|c|c|c|c|c|c|c|c|c|c|}
\hline \multirow{3}{*}{\multicolumn{2}{|c|}{ Kernel Batch No. }} & \multirow{4}{*}{\multicolumn{2}{|c|}{$\begin{array}{c}\text { Total } \\
\text { Diam (a) } \\
(\mu \mathrm{m})\end{array}$}} & \multirow{3}{*}{\multicolumn{3}{|c|}{$\begin{array}{l}\text { Particle } \\
\text { Density } \\
\left(\mathrm{Mg} / \mathrm{m}^{3}\right)\end{array}$}} & \multirow{2}{*}{\multicolumn{5}{|c|}{ Chemical Composition }} & \multicolumn{5}{|c|}{ Particle Defects } & \multirow{4}{*}{$\begin{array}{c}\text { Th } \\
\text { Contanination (c) } \\
(\mathrm{kgTh} / \mathrm{kgTh})\end{array}$} & \multirow{4}{*}{$\begin{array}{c}\text { Fission } \\
\text { Gas } \\
\text { Release(f } \\
\quad(R / B) \\
\end{array}$} \\
\hline & & & & & & & & & & & & \multirow{2}{*}{\multicolumn{2}{|c|}{$\begin{array}{l}\text { Fraction } \\
\text { Defective } \\
\text { Coating }\end{array}$}} & \multirow{3}{*}{$\begin{array}{c}\text { Excessive } \\
\text { Fuel } \\
\text { Dispersion }(a, e)\end{array}$} & \multirow{2}{*}{\multicolumn{2}{|c|}{$\begin{array}{c}\text { Fraction of } \\
\text { Missing or Incomplete } \\
\text { Coating }(a, e) \\
\end{array}$}} & & \\
\hline & & & & & & & \multirow[b]{2}{*}{$\begin{array}{l}\text { Th } \\
\text { Content } \\
\text { (wt \%) }\end{array}$} & \multirow[b]{2}{*}{$\begin{array}{c}\left.{ }_{(w t}^{C} \%\right) \\
\end{array}$} & \multirow[b]{2}{*}{$\begin{array}{c}s i \\
\text { (wt \%) }\end{array}$} & \multicolumn{2}{|c|}{$\begin{array}{c}\text { Impurities } \\
(\mathrm{ppm}-\mathrm{wt})\end{array}$} & & & & & & & \\
\hline $\begin{array}{c}\text { Data } \\
\text { Retrieval }\end{array}$ & Manufacturer & & & $\begin{array}{c}\mathrm{Hg} \\
\text { Porosimeter } \\
\end{array}$ & $\begin{array}{c}\text { Liquid } \\
\text { Mean } \\
\end{array}$ & \begin{tabular}{|l} 
Gradient \\
SD \\
\end{tabular} & & & & $\mathrm{Fe}$ & $\begin{array}{c}\text { Be- } \\
\text { Equivalent (b) }\end{array}$ & $\begin{array}{c}\text { Burn-Leach } \\
\text { Test (c) }\end{array}$ & $\begin{array}{c}\text { Burn-Visual } \\
\text { Test (d) }\end{array}$ & & $\begin{array}{l}\text { Buffer } \\
\text { and /or } \\
\text { IPyc }\end{array}$ & OPyc & & \\
\hline $6252-07-025$ & $6780-13-0$ & 822 & 28.0 & 3.32 & 3.34 & 0.139 & 43.41 & 35.89 & 13.39 & 72 & 1.8 & $2.9 \times 10^{-4}$ & $1.0 \times 10^{-4}$ & 0 & 0 & 0 & $1.1 \times 10^{-5}$ & $1.6 \times 10^{-5}$ \\
\hline $6252-13-010$ & $6252-12 Q-02 A$ & 811 & 268 & 3.39 & 3.43 & 0.139 & 45.00 & 33.94 & 14.85 & 74 & 2.0 & $4.3 \times 10^{-4}$ & $9.7 \times 10^{-4}$ & 0 & .0 & $3.1 \times 10^{-4}$ & $4.9 \times 10^{-5}$ & $1.2 \times 10^{-5}$ \\
\hline $6252-14-010$ & $6252-120-07 \mathrm{~A}$ & 808 & 28.9 & 3.37 & 3.42 & 0.163 & 46.20 & 35.25 & 13.20 & 60 & 2.0 & $2.4 \times 10^{-3}$ & $4.1 \times 10^{-3}$ & 0 & $3.0 \times 10^{-4}$ & 0 & $8.3 \times 10^{-5}$ & $4.2 \times 10^{-5}$ \\
\hline $6252-14-020$ & $6780-141-02 B$ & 791 & 25.6 & 3.49 & 3.54 & 0.147 & 47.60 & 33.50 & 12.75 & 71 & 1.7 & $1.1 \times 10^{-3}$ & $1.5 \times 10^{-3}$ & 0 & $1.3 \times 10^{-3}$ & 0 & $6.1 \times 10^{-4}$ & $3.9 \times 10^{-5}$ \\
\hline $6252-15-0.10$ & $6252-1.2 Q-08 \mathrm{~A}$ & 807. & 30.5 & 3.36 & 3.39 & 0.113 & 45.85 & 34.73 & 13.58 & 54 & 2.1 & $1.9 \times 10^{-4}$ & $2.7 \times 10^{-4}$ & 0 & 0 & 0 & $4.9 \times 10^{-6}$ & $4.0 \times 10^{-6}$ \\
\hline $6252-16-010$ & $6252-12 Q-08 B$ & 816 & 29.2 & 3.37 & 3.39 & 0.160 & 44.78 & 36.37 & 13.42 & 60 & 2.0 & $8.9 \times 10^{-5}$ & $3.7 \times 10^{-4}$ & 0 & 0 & 0 & $9.8 \times 10^{-6}$ & $2.5 \times 10^{-6}$ \\
\hline $6252-17-010$ & $6780-1 \cdot 47-03 B$ & 882 & 51.6 & 2.98 & 3.03 & 0.193 & 41.6 .5 & 39.00 & 14.16 & 140 & 2.3 & $1.3 \times 10^{-3}$ & $1.2 \times 10^{-3}$ & 0 & $3.3 \times 10^{-4}$ & 0 & $2.1 \times 10^{-5}$ & $2.1 \times 10^{-5}$ \\
\hline $6252-20-010$ & $6780-147-03 \mathrm{~A}$ & 874 & 44.1 & 2.98 & 3.09 & 0.157 & 41.79 & $39.65^{\circ}$ & 13.55 & 87 & 2.3 & $2.9 \times 10^{-3}$ & $2.3 \times 10^{-3}$ & 0 & $1.3 \times 10^{-3}$ & 0 & $2.5 \times 10^{-4}$ & $7.3 \times 10^{-5}$ \\
\hline
\end{tabular}

(a) Measured by contact $\mathrm{X}$-radiography.

(b) The following elements were measured by spectrographic analysis:

$\mathrm{Al}, \mathrm{Be}, \mathrm{Cd}, \mathrm{Fe}, \mathrm{Mo}, \mathrm{P}, \mathrm{Sn}, \mathrm{V}, \mathrm{B}, \mathrm{Bi}, \mathrm{Cr}, \mathrm{Mg}, \mathrm{Na}, \mathrm{Pb}, \mathrm{Sr}, \mathrm{Zn}, \mathrm{Ba}, \mathrm{Ca}, \mathrm{Mn}, \mathrm{Ni}, \mathrm{Si}, \mathrm{Tl}$.

(c) $25 \mathrm{~g}$ samples leached for $24 \mathrm{hr}$ in ultraleach.

(d) $\geq 10 \mathrm{~g}$ sample examined.

(e) Measured on 23000 particles.

(f) Release rate/birth rate for $\mathrm{Kr}-85 \mathrm{~m}$ at $1100^{\circ} \mathrm{C}$ for $\sim 20 \mathrm{~g}$ samples. 
THIS PAGE

\section{WAS INTENTIONALLY LEFT BLANK}


TABLE $3-12$ I
PARTICLE SAMPLES FOR CAPSULE HT-34

\begin{tabular}{|c|c|c|c|c|c|c|c|c|c|c|c|c|c|c|c|c|c|c|c|c|c|}
\hline \multirow{3}{*}{$\begin{array}{c}\text { Sample } \\
\text { Batch No. }\end{array}$} & \multirow{2}{*}{\multicolumn{2}{|c|}{$\left.\begin{array}{c}\text { Kernel Diam }(a) \\
(\mu \mathrm{m})\end{array}\right)$}} & \multicolumn{3}{|c|}{ Buffer Coating } & \multicolumn{3}{|c|}{ IPyC Coating } & \multicolumn{3}{|c|}{ SiC Coating } & \multicolumn{5}{|c|}{ OPyc coating } & \multicolumn{4}{|c|}{ Total Particle } & \multirow{3}{*}{$\begin{array}{r}\begin{array}{r}\text { Radiograph } \\
\text { Plate No. }\end{array} \\
\end{array}$} \\
\hline & & & \multicolumn{2}{|c|}{$\begin{array}{c}\text { Calculated Thickness }(\mathrm{a}, \mathrm{b}) \\
(\mu \mathrm{m})\end{array}$} & \multirow{2}{*}{$\begin{array}{c}\text { Coating } \\
\text { Rate } \\
(\mu \mathrm{m} / \mathrm{min}) \\
\end{array}$} & \multicolumn{2}{|c|}{$\begin{array}{c}\text { Calculated Thickness }(\mathrm{a}, \mathrm{c}) \\
(\mathrm{\mu m})\end{array}$} & \multirow{2}{*}{$\begin{array}{c}\text { Coating } \\
\text { Rate } \\
(\mu \mathrm{m} / \mathrm{min}) \\
\end{array}$} & \multicolumn{2}{|c|}{$\begin{array}{c}\text { Thickness (a) } \\
(\mu \mathrm{m})\end{array}$} & \multirow{2}{*}{$\begin{array}{c}\text { Coating } \\
\text { Rate } \\
\text { ( } \mu \mathrm{m} / \mathrm{m} \text { ) } \\
\end{array}$} & \multicolumn{2}{|c|}{$\begin{array}{c}\text { Thickness }(\mathrm{a}) \\
(\mu \mathrm{m})\end{array}$} & \multirow{2}{*}{$\begin{array}{c}\text { Coating } \\
\text { Rate } \\
(\mu \mathrm{m} / \mathrm{min}) \\
\end{array}$} & \multicolumn{2}{|c|}{$\mathrm{BAF}_{\mathrm{o}}(\mathrm{d})$} & \multicolumn{2}{|c|}{$\begin{array}{c}\underset{\operatorname{diam}(a)}{(\mu \mathrm{m})} \\
\end{array}$} & \multicolumn{2}{|c|}{$\begin{array}{c}\text { Density }(\mathrm{e}) \\
\left(\mathrm{Mg} / \mathrm{m}^{3}\right)\end{array}$} & \\
\hline & Mean & SD & Mean & SD & & Mean & SD & & Mean & SD & & Mean & SD & & Mean & SD & Mean & SD & Mean & $\mathrm{sr}$ & \\
\hline $6252-07-0262-001$ & 449 & 4.8 & 60 & 7.1 & 9.68 & 39 & 4.8 & 4.4 & 38 & 2.5 & 0.17 & 45 & 5.7 & 5.0 & ND (f) & ND & 816 & 15.3 & 3.35 & 0.002 & LB720 \\
\hline $6252-07-0262-002$ & 449 & 4.8 & 57 & 8.0 & 9.19 & 39 & 4.8 & 4.4 & 37. & 3.1 . & 0.16 & 41 & .6 .1 & 4.6 & ND & ND & 808 & 19.2 & 3.35 & 0.002 & J.R719 \\
\hline $6252-13-0161-001$ & 447 & 7.4 & 57 & 6.4 & 9.50 & 32 & 4.1 & 4.1 & 43 & 2.3 & 0.33 & 48 & 6.0 & 8.3 & 1.031 & 0.0025 & 808 & 18.8 & 3.39 & 0.017 & LB734 \\
\hline $6252-13-0171-001$ & 443 & 7.7 & 79 & 9.1 & 13.17 & 32 & 4.1 & 4.1 & 43 & 2.5 & 0.33 & 49 & 5.2 & 8.5 & 1.031 & 0.0025 & 848 & 20.3 & 3.13 & 0.021 & LB728 \\
\hline $6252-14-0161-001$ & 448 & 7.6 & 63 & 7.0 & 9.69 & 32 & 4.4 & 4.0 & 37 & 2.6 & 0.31 & 44 & 4.8 & 7.6 & 1.047 & 0.0046 & 798 & 17.2 & 3.42 & 0.039 & LB727 \\
\hline $6252-14-0161-002$ & 447 & 6.8 & 62 & 7.3 & 9.54 & 32 & .4 .4 & 4.0 & 37 & 2.3 & 0.31 & 44 & 4.6 & 7.6 & 1.047 & 0.0046 & 796 & 19.0 & 3.42 & 0.039 & LB726 \\
\hline $6252-14-0171-001$ & 451 & 10.0 & 35 & 5.9 & 5.38 & 32 & 4.4 & 4.0 & 35 & 2.5 & 0.29 & 41 & 4.2 & 7.1 & 1.047 & 0.0046 & 736 & 19.0 & 3.93 & 0.041 & LB730 \\
\hline $6252-14-0181-001$ & 445 & 7.1 & 91 & 9.0 & 14.00 & 32 & 4.4 & 4.0 & 37 & 2.6 & 0.31 & 47 & 5.6 & 8.1 & 1.047 & 0.0046 & 854 & 20.8 & 3.06 & 0.026 & LB729 \\
\hline $6252-14-0261-001$ & 455 & 7.1 & 27 & 6.0 & 4.35 & 35 & 4.9 . & 4.7 & 35 & 3.0 & 0.27 & 40 & 4.9 & 7.1 & ND & ND & 729 & 17.5 & 3.96 & 0.011 & LB736 \\
\hline $6252-14-027 \cdot 1-001$ & 443 & 8.7 & 86 & 9.0 & 13.87 & 35 & 4.9 & 4.7 & 38 & 3.2 & 0.29 & .45 & 5.7 & 8.0 & ND & ND & 849 & 24.4 & 3.11 & 0.062 & LB732 \\
\hline $6252-15-0161-001$ & 450 & 7.2 & 56 & 6.9 & 9.33 & 36 & 4.4 & 4.2 & 38 & 2.1 & 0.32 & 45 & 5.1 & 5.0 & 1.035 & 0.0036 & 798 & 13.9 & 3.38 & 0.022 & LB725 \\
\hline $6252-15-0171-001$ & 448 & 7.9 & 84 & 7.7 & 14.00 & 36 & 4.4 & 4.2 & 39 & 2.6 & 0.33 & 50 & 5.5 & 5.6 & 1.035 & 0.0036 & 865 & 21.0 & 3.07 & 0.020 & LB731 \\
\hline $6252-16-0161-001$ & 451 & 6.8 & 57 & 6.5 & 9.50 & 36 & 4.4 & 4.2 & 39 & 2.0 & 0.33 & 48 & 5.8 & 5.3 & 1.052 & 0.0056 & 813 & 17.2 & 3.41 & 0.011 & LB724 \\
\hline $6252-17-0161-001$ & 451 & 7.0 & 80 & 9.2 & 11.43 & 40 & 5.1 & 4.2 & 37 & 4.8 & 0.24 & 45, & 16.2 & 5.0 & ND & ND & 858 & 23.1 & 3.11 & 0.017 & LB721 \\
\hline $6252-20-0161-001$ & 450 & 7.3 & 83 & 8.5 & 11.86 & 40 & 5.1 & 4.2 & 36 & 3.7 & 0.23 & 47 & 5.6 & 8.4 & ND & ND & 862 & 22.1 & 3.06 & 0.030 & LB723 \\
\hline $6252-20-0161-002$ & 452 & 7.0 & 82 & $8.1^{\circ}$ & 11.71 & 40 & 5.1 & $4: 2$ & 35 & 3.7 & 0.23 & $45^{\circ}$ & 5.4 & 8.0 & ND & ND & 854 & 23.3 & 3.06 & 0.030 & LB722 \\
\hline
\end{tabular}

(a) Measured by contact $\mathrm{X}$-radiography of $\geq 200$ particles.

(b) Calculated (after SiC coating deposited) by subtracting the IPyC thickness from measured buffer and IPyC thickness.

(c) Measured at BISO stage on parent batch.

(d) Measured with Seibersdorf unit at GA using $24-\mu \mathrm{m}-\mathrm{spot}$ on 50 companion particles.

(e) Measured by a 1iquid gradient technique on companion sample.

(f) ND - Not determined. 
THIS PAGE

\section{WAS INTENTIONALLY LEFT BLANK}


TABLE 3-13

SUMMARY OF TRISO $\mathrm{ThO}_{2}$ PARTICLE SAMPLES FOR CAPSULE HT- 34

\begin{tabular}{|c|c|c|c|c|c|c|c|c|c|c|c|c|c|c|c|c|c|c|c|}
\hline \multirow[b]{3}{*}{ Sample Batch No. } & \multirow[b]{3}{*}{ 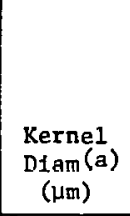 } & \multirow{2}{*}{\multicolumn{7}{|c|}{ Coatings }} & \multirow{2}{*}{\multicolumn{7}{|c|}{ OPyC }} & \multicolumn{4}{|c|}{ Total coated Particle } \\
\hline & & & & & & & & & & & & & & & & \multirow[b]{2}{*}{$\underset{(\mathrm{m})}{\operatorname{Dtam}(\mathrm{a})}$} & \multirow[b]{2}{*}{$\begin{array}{c}\text { Defective } \\
\text { sic } \\
\text { Caat Ing (c,e) } \\
\end{array}$} & \multirow[b]{2}{*}{$\begin{array}{c}\text { Thorfum } \\
\text { Contamination }(\mathrm{c}, \mathrm{f}) \\
(\mathrm{kg} T \mathrm{Th} / \mathrm{kgTh})\end{array}$} & \multirow[b]{2}{*}{$\begin{array}{c}\text { Fisston } \\
\text { Gas } \\
\text { Release (c,g) } \\
(\mathrm{R} / \mathrm{B}) \\
\end{array}$} \\
\hline & & $\underset{(\mathrm{mm})}{\operatorname{TTh1.kness}(\mathrm{a}, \mathrm{b})}$ & $\begin{array}{c}\text { Dengstyy (c) } \\
\left(\mathrm{Mg}_{\mathrm{g} / \mathrm{m} 3)}\right.\end{array}$ & $\underset{(\mu \mathrm{m})}{\text { Th1 ckness }(\mathrm{c}, \mathrm{d})}$ & $\begin{array}{l}\text { Liquid } \\
\text { Cradient } \\
\text { Density (c) } \\
\left(\mathrm{Mg}_{\left.\mathrm{g} / \mathrm{m}^{3}\right)}\right)\end{array}$ & $\begin{array}{c}\text { Thickness (a) } \\
(\mu \mathrm{mm})\end{array}$ & \begin{tabular}{|l} 
Densityy (c) \\
$\left(\mathrm{Mg}_{\mathrm{g}} / \mathrm{m}^{3}\right)$
\end{tabular} & 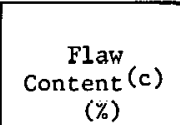 & $\underset{\substack{\text { Thyckness } \\
(\mathrm{\mu m})}}{(\mathrm{a})}$ & \multicolumn{2}{|c|}{$\begin{array}{c}\text { Density (c) } \\
\left(\mathrm{Mg} / \mathrm{m}^{3}\right)\end{array}$} & $\mathrm{BAF}_{\mathrm{o}}(\mathrm{c})$ & $\begin{array}{c}\text { Accessitb1e } \\
\text { Porosity (c) } \\
(\mathrm{m} 1 / \mathrm{kg})\end{array}$ & $\begin{array}{l}\text { Coating } \\
\text { Rate (a) } \\
(a \mathrm{~m} / \mathrm{min})\end{array}$ & $\begin{array}{l}\text { Coating } \\
\text { Diluivent } \\
\text { Gas }(c)\end{array}$ & & & & \\
\hline $6252-07-0262-001$ & 449 & 60 & 1.12 & 39 & 1.93 & 38 & 3.21 & 38 & 45 & 1.80 & 1.65 & 1.027 & 48 & 5.0 & Ar & 816 & $1.0 \times 10^{-4}$ & $1.1 \times 10^{-5}$ & $1.6 \times 10^{-5}$ \\
\hline $6252=07-0262-002$ & 449 & 57 & 1.12 & 39 & 1.93 & 37 & 3.21 & 38 & 41 & 1.80 & 1.65 & 1.027 & 48 & 4.6 & $\mathrm{Ar}$ & 808 & $1.0 \times 10^{-4}$ & $1.1 \times 10^{-5}$ & $1.6 \times 10^{-5}$ \\
\hline 6252-13-0161-001 & 447 & 57 & 1.13 & 32 & 1.86 & 43 & 3.22 & 16 & 48 & 1.79 & 1.61 & 1.031 & 57 & 8.3 & $\mathrm{H}_{2}$ & 808 & $9.7 \times 10^{=1}$ & $4.9 \times 10^{-5}$ & $1.2 \times 10^{-5}$ \\
\hline 6252-13-0171-001 & 443 & 79 & 1.13 & 32 & 1.86 & 43 & 3.22 & 16 & 49 & 1.79 & 1.61 & 1.031 & 57 & 8.5 & $\mathrm{H}_{2}^{2}$ & 848 & $9.7 \times 10^{-4}$ & $4.9 \times 10^{-5}$ & $1.2 \times 10^{-5}$ \\
\hline 6252-14-0161-001 & 448 & 63 & 1.10 & 32 & 1.84 & 37 & 3.22 & 9 & 44 & 1.97 & 1.76 & 1.041 & 57 & 7.6 & $\mathrm{H}_{2}^{2}$ & 798 & $4.1 \times 10^{-3}$ & $8.3 \times 10^{-5}$ & $4.2 \times 10^{-5}$ \\
\hline $6252-14-0161-002$ & 447 & 62 & 1.10 & 32 & 1.84 & 37 & 3.22 & 9 & 44 & 1.97 & 1.76 & 1.041 & 57 & 7.6 & $\mathrm{H}_{2}^{2}$ & 796 & $4.1 \times 10^{-3}$ & $8.3 \times 10^{-5}$ & $4.2 \times 10^{-5}$ \\
\hline 6252-14-0171-001 & 451 & 35 & 1.10 & 32 & 1.84 & 35 & 3.22 & 9 & 41 & 1.97 & 1.76 & 1.041 & 57 & 7.1 & $\mathrm{H}_{2}^{2}$ & 736 & $4.1 \times 10^{-3}$ & $8.3 \times 10^{-5}$. & $4.2 \times 10^{-5}$ \\
\hline 6252-14-0181-001 & 445 & 91 & 1.10 & 32 & 1.84 & 37 & 3.22 & 9 & 47 & 1.97 & 1.76 & 1.041 & 57 & 8.1 & $\mathrm{H}_{2}^{2}$ & 854 & $4.1 \times 10^{-3}$ & $8.3 \times 10^{-5}$ & $4.2 \times 10^{-5}$ \\
\hline $6252-14-0261-001$ & 455 & 27 & 1.08 & 35 & 1.83 & 35 & 3.21 & 29 & 40 & 1.97 & 1.79 & 1.040 & 54 & 7.1 & $\mathrm{H}_{2}^{2}$ & 729 & $1.5 \times 10^{-3}$ & $6.1 \times 10^{-4}$ & $3.9 \times 10^{-5}$ \\
\hline $6252-14-0271-001$ & 443 & 86 & 1.08 & 35 & 1.83 & 38 & 3.21 & 29 & 45 & $\cdot 1.97$ & 1.79 & 1.040 & 54 & 8.0 & $\mathrm{H}_{2}$ & 849 & $1.5 \times 10^{-3}$ & $6.1 \times 10^{-4}$ & $3.9 \times 10^{-5}$ \\
\hline $6252-15-0161-001$ & 450 & 56 & 1.07 & 36 & 1.85 & 38 & 3.22 & 12 & 4.5 & 1.81 & 1.68 & 1.033 & 21 & 5.0 & $\mathrm{H}_{2}$ & 798 & $2.7 \times 10^{-4}$ & $4.9 \times 10^{-6}$ & $4.0 \times 10^{-6}$ \\
\hline $6252-15-0171-001$ & 448 & 84 & 1.07 & 36 & 1.85 & 39 & 3.22 & 12 & 50 & 1.81 & 1.68 & 1.033 & 21 & 5.6 & $\mathrm{H}_{2}^{2}$ & 865 & $2.7 \times 10^{-4}$ & $4.9 \times 10^{-6}$ & $4.0 \times 10^{-6}$ \\
\hline $6252-16-0161-001$ & 451 & 57 & 1.07 & -36 & 1.85 & 39 & 3.21 & 12 & 48 & 1.96 & 1.82 & 1.048 & 25 & 5.3 & $\mathrm{H}_{2}^{2}$ & 813 & $3.7 \times 10^{-4}$ & $9.8 \times 10^{-6}$ & $2.5 \times 10^{-6}$ \\
\hline $6252-17-0161-001$ & 451 & 80 & 0.93 & 40 & 1.79 & 37 & 3.22 & 6 & 45 & 1.95 & 1.80 & 1.049 & 28 & 5.0 & $\mathrm{H}_{2}^{2}$ & 858 & $1.2 \times 10^{-3}$ & $2.1 \times 10^{-5}$ & $2.1 \times 10^{-5}$ \\
\hline $6252-20-0161-001$ & 450 & 83 & 0.93 & 40 & 1.79 & 36 & 3.21 & 6 & 47 & 1.98 & 1.74 & 1.041 & 59 & 8.4 & $\mathrm{H}_{2}^{2}$ & 862 & $2.3 \times 10^{-3}$ & $2.5 \times 10^{-4}$ & $7.3 \times 10^{-5}$ \\
\hline $6252-20-0161-002$ & 452 & 82 & 0.93 & 40 & 1.79 & 35 & 3.21 & 6 & 45 & 1.98 & 1.74 & 1.041 & 59 & 8.0 & $\mathrm{H}_{2}^{2}$ & 854 & $2.3 \times 10^{-3}$ & $2.5 \times 10^{-4}$ & $7.3 \times 10^{-5}$ \\
\hline
\end{tabular}

(a) Measured or calculated on capsule sample.

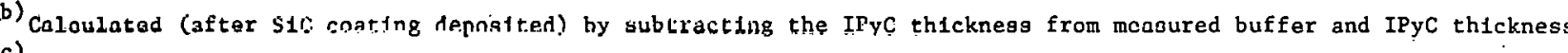

(d) Measured or calculated on parent batch.

(e) Measured at BISO stage.

(e) Measured by burn-visual examination technique.

(8) Leached for 24 hr th ultraleach.

$\mathrm{Kr}-85 \mathrm{~m}$ at $1100^{\circ} \mathrm{C}$. 
THIS PAGE

\section{WAS INTENTIONALLY LEFT BLANK}


TABLE 3-14

COMPARISON OF HT-34 PARENT BATCHES WITH CAPSULE SPECIMENS

\begin{tabular}{|c|c|c|c|c|c|c|c|c|c|c|c|c|c|c|c|c|c|}
\hline \multirow[b]{2}{*}{ Sample 3atch No. } & \multirow{2}{*}{$\begin{array}{r}\text { Density } \\
\text { Fraction } \\
\end{array}$} & \multicolumn{2}{|c|}{$\begin{array}{c}\text { Kerr.el Diam } \\
(\mu \mathrm{m})\end{array}$} & \multicolumn{2}{|c|}{$\begin{array}{c}\text { Buffer } \\
\text { Thickness } \\
(\mu \mathrm{m})\end{array}$} & \multirow{2}{*}{$\begin{array}{c}\begin{array}{c}\text { IPyC } \\
\text { Thickness } \\
(\mu \mathrm{m})\end{array} \\
\text { Mean } \\
\end{array}$} & \multicolumn{2}{|c|}{$\begin{array}{c}\text { Sic } \\
\text { Thickness } \\
(\mu \mathrm{m})\end{array}$} & \multicolumn{2}{|c|}{$\begin{array}{c}\text { OPyC } \\
\text { Thickness } \\
(\mu \mathrm{m})\end{array}$} & \multicolumn{2}{|c|}{$\begin{array}{c}\text { Total } \\
\text { Particle } \\
\text { Diam } \\
(\mu \mathrm{m})\end{array}$} & \multirow{2}{*}{$\begin{array}{c}\text { Radiograph } \\
\text { Plate } \\
\text { No. } \\
\end{array}$} & \multirow{2}{*}{$\begin{array}{c}\text { No. } \\
\text { Particles }\end{array}$} & \multirow{2}{*}{$\begin{array}{c}\text { Liquid } \\
\text { Gradient } \\
\text { Density } \\
\left(\mathrm{Mg} / \mathrm{m}^{3}\right)\end{array}$} & \multicolumn{2}{|c|}{$\begin{array}{l}B A F_{0} \\
(\mu m)\end{array}$} \\
\hline & & Mear: & 0 & Mean & $c$ & & Mean & $\sigma$ & Mean & $\sigma$ & Mean & 0 & & & & Mean & $\sigma$ \\
\hline $6252-07-0200(a)$ & -- & 452.60 & $8.3 \hat{\imath}$ & 62.93 & 11.35 & 39 & 37.69 & 3.27 & $4: .50$ & 6.19 & 821.80 & 28.02 & -- & -- & 3.340 & 1.027 & 0.0030 \\
\hline $6252-07-0262-001$ & Mominal & 448.55 & 4.30 & 59.93 & 7.13 & 39 & 38.36 & 2.54 & 45.28 & 5.73 & 815.62 & 15.31 & L.8720 & 57 & 3.340 & ND & ND \\
\hline $6252-07-0262-002$ & Mominal & 448.53 & 4.33 & 56.50 & 8.02 & 39 & 37.21 & 3.06 & $4 i .42$ & 6.11 & 807.97 & 19.23 & LB719 & 81 & 3.340 & ND & ND \\
\hline $6252-13-0100(a)$ & -- & 453.53 & 8.33 & 55.03 & 9.57 & 32 & 42.46 & 2.33 & 49.92 & 4.87 & 810.86 & 26.76 & -- & -- & 3.430 & 1.031 & 0.0027 \\
\hline $6252-13-0161-001$ & Nominal & 447.20 & 7.43 & 56.87 & 6.41 & 32 & 43.45 & 2.26 & 48.25 & 6.04 & 808.45 & $18.85^{\circ}$ & LB7 34 & 57 & 3.391 & 1.031 & 0.0025 \\
\hline $6252-13-0171-001$ & Low & $442 . ? 1$ & 7.72 & 79.01 & 9.06 & 32 & 42.78 & 2.50 & 49.12 & 5.18 & 848.37 & 20.29 & LB728 & 85 & 3.126 & 1.031 & 0.0025 \\
\hline $6252-14-0100(a)$ & -- & 451.23 & 9.12 & 62.75 & $12.0 / 4$ & 32 & 37.35 & 2.48 & 47.60 & 5.17 & 808.01 & 28.93 & -- & -- & 3.420 & 1.041 & 0.0042 \\
\hline $6252-14-0161-002$ & Nomíral & 447.73 & 7.59 & 62.66 & 7.04 & 32 & 37.00 & 2.61 & 44.39 & 4.84 & 797.66 & 17.23 & LB727 & 57 & 3.421 & 1.047 & 0.0046 \\
\hline $6252-14-0161-002$ & Nomiral & 447.38 & 6.82 & 62.22 & 7.27 & 32 & 36.64 & 2.34 & 44.49 & 4.60 & 796.18 & 18.99 & LB726 & 81 & 3.421 & 1.047 & 0.0046 \\
\hline 6252-14-0171-001 & High & 451.30 & 9.98 & 34.95 & 5.87 & 32 & 35.21 & $2.51^{\circ}$ & 40.57 & 4.21 & 735.63 & 18.79 & LB730 & 56 & 3.929 & 1.047 & 0.0046 \\
\hline $6252-16-0181-001$ & Low & 444.53 & 7.08 & 91.02 & 8.97 & 32 & 36.78 & 2.59 & 46.62 & 5.60 & 854.44 & 20.77 & LB729 & 82 & 3.057 & 1.047 & 0.0046 \\
\hline $6252-14-0200^{(a)}$ & -- & 450.50 & 8.07 & 54.63 & 10.66 & 35 & 35.70 & 2.73 & 43.25 & 5.71 & 791.30 & 25.63 & -- & - & 3.540 & 1.040 & 0.0048 \\
\hline $6252-14-0261-001$ & $: \mathrm{Iigh}$ & 454.59 & 7.106 & 27.32 & 6.02 & 35 & 34.77 & 2.95 & 39.63 & 4.86 & 728.86 & 17.48 & LB736 & 56 & 3.958 & ND & $\mathrm{ND}$ \\
\hline $6252-14-0271-001$ & Low & 442.38 & 8.71 & 86.32 & 8.99 & 35 & 38.42 & 3.24 & 45.13 & 5.69 & 848.58 & 24.44 & LB732 & 83 & 3.107 & ND & ND \\
\hline $6252-1 \leq-0100(a)$ & -- & 455.33 & 8.43 & 54.04 & 11.37 & 36 & 38.08 & 2.35 & 48.00 & 6.29 & 807.28 & 30.47 & -- & -- & 3.394 & 1.033 & 0.0032 \\
\hline $6252-1 \leq-0161-001$ & Nominal. & 449.74 & 7.23 & 55.83 & 6.87 & 36 & 37.77 & 2.06 & 45.21 & 5.13 & 797.93 & 13.91 & LB725 & 56 & 3.380 & 1.035 & 0.0036 \\
\hline $6252-15-0171-001$ & Low & 448. 50 & 7.94 & 84.49 & 7.72 & 36 & 39.09 & 2.58 & 49.95 & 5.45 & 864.82 & 21.04 & LB731 & 81 & 3.070 & 1.035 & 0.0036 \\
\hline $6252-16-0100^{(a)}$ & -- & 454.52 & 8.43 & 57.84 & 12.29 & 36 & 38.69 & 2.04 & 49.97 & 5.65 & 815.70 & 29.18 & -- & -- & 3.392 & 1.048 & 0.0048 \\
\hline $6252-16-0161-001$ & Nominal & 450.84 & 6.77 & 57.49 & 6.48 & 36 & 38.51 & 2.02 & 47.84 & 5.81 & 813.26 & 17.19 & LB724 & 56 & 3.413 & 1.052 & 0.0056 \\
\hline $6252-1^{7}-0100^{(a)}$ & -- & 454.50 & 8.93 & 89.71 & $2 \bar{z} .32$ & 40 & 36.27 & $3 . \cong 5$ & 43.60 & 7.06 & 882.10 & 51.61 & -- & -- & 3.030 & 1.049 & 0.0043 \\
\hline $6252-17-0161-001$ & Nominal & 451.24 & 7.02 & 79.61 & $\varsigma .22$ & 40 & 36.92 & 4.77 & 44.53 & 6.19 & 858.43 & 23.11 & LB721 & 81 & 3.109 & ND & ND \\
\hline $6252-20-0100(a)$ & -- & 455.85 & 8.63 & 85.77 & $1 \varsigma .17$ & 40 & 36.60 & 3.37 & 43.36 & 5.76 & 873.60 & 44.05 & -- & -- & 3.090 & 1.041 & 0.0046 \\
\hline $6252-20-0161-001$ & Nominal. & 449.77 & 7.27 & 82.97 & $\varepsilon .49$ & 40 & 36.02 & 3.68 & 47.34 & 5.59 & 862.12 & 22.13 & LB723 & 56 & 3.057 & ND & ND \\
\hline $6252-23-0161-002$ & Nominal & 451.55 & 6.98 & 82.32 & $\varepsilon .13$ & 40 & 35.24 & 3.69 & 45.04 & 5.36 & 854.23 & 23.26 & L.B722 & 80 & 3.057 & ND & ND \\
\hline
\end{tabular}

Note: $\begin{aligned} \sigma & =\text { standard deviation } \\ \text { ND } & =\text { not determined }\end{aligned}$ 
-

.

.

:
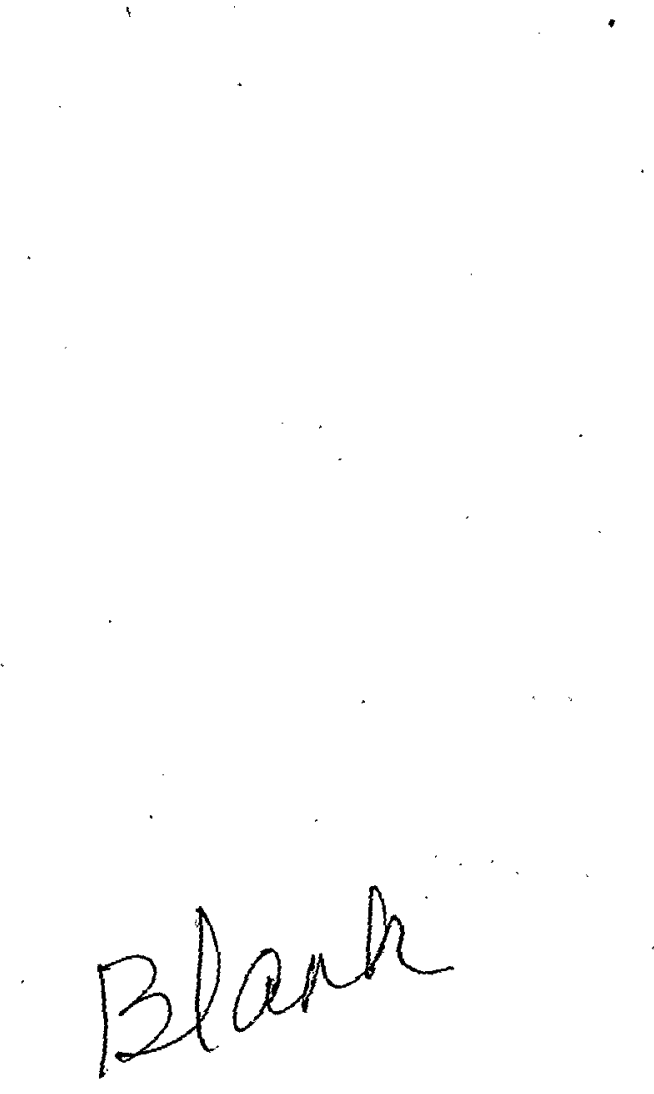

\&

$$
3-54
$$




\section{CAPSULE PARAMETERS}

\subsection{OPERATING HISTORY}

Capsule HT-34 was inserted into the C-6 target position of the HFIR reactor on July 25 and removed on November 23, 1977. The capsule was irradiated for five cycles for a total of 2686 hs at $100-M W$ power. The operating history (Ref. 14) of the capsule is given in Table 4-1.

\subsection{FLUENCE AND BURNUP ANALYSIS}

The fast-fluence data for capsule HT-34 are reported in Table 4-2; they were calculated by ORNL (Ref. 14). The fluence ranged from 5.1 to 8.2 $x 10^{29} \mathrm{n} / \mathrm{m}^{2}$ (E > $29 \mathrm{fJ}$ ) HTGR for the low-temperature magazine and 9.2 to $10.2 \times 10^{25} \mathrm{n} / \mathrm{m}^{2}(\mathrm{E}>29 \mathrm{fJ})_{\mathrm{HTGR}}$ for the high-temperature magazine.

The burnups of the irradiated $\mathrm{ThO}_{2}$ samples were calculated by ORNL (Ref. 14) and GA (Ref. 15), and the values are reported in Table 4-2. The GA calculations were based on reaction rates determined from the analytical burnup measurements of capsule samples HT-31 and HT-33. The details of this analysis are given in Ref. 16. The GA values were $8 \%$ to $12 \%$ lower than the ORNL values for the $1200^{\circ} \mathrm{C}$ samples and $2 \%$ lower to $4 \%$ higher for the $1450^{\circ} \mathrm{C}$ samples. This is good agreement, considering the numerous uncertainties associated with burnup calculations. The GA values will be reported in the following sections of this document.

\subsection{THERMAL ANALYSIS}

Actual temperatures were not monitored during the 1rradiation. ORNL performed a detailed thermal analysis (Ref. 17) using the thermal modeling code HTCAP 1, which is a modified version of HTCAP (Ref. 18). This code 
calculates total heat generation within the capsule, performs a modified, one-dimensional heat transfer analysis, then predicts the maximum particle surface operating temperature at one-day intervals during each irradiation cycle.

The results of the thermal analysis (Ref. 19) are plotted in Fig. 4-1, which shows the graphite annulus temperature, particle surface temperature, and power per particle. Tables $4-3$ and $4-4$ present the time-averaged particle surface temperature and power/particle/cycle for each sample, respectively. The results showed that the temperatures increased with time and that the highest maximum surface temperature for the last cycle was $1340^{\circ} \mathrm{C}$ for the low-temperature magazine and $1530^{\circ} \mathrm{C}$ for the other magazine. The time-averaged maximum surface temperatures were $1180^{\circ}$ to $1250^{\circ} \mathrm{C}$ and $1430^{\circ} \mathrm{C}$ to $1450^{\circ} \mathrm{C}$ for the two magazines. The power/particle for the last cycle averaged 0.29 to 0.42 watts for the low-temperature magazine and 0.48 to 0.51 watts for the high-temperature magazine.

Based on the thermal analysis data supplied by ORNL, the time-volume averaged temperature of the kernel was calculated (Ref. 20) for each cycle and is summarized in Table 4-5. Particle dimensions and conductivities, surface temperature, and power generation for each sample were input into the calculations. The analysis was modeled after a general study made on the temperature distribution inside a TRISO particle (Ref. 21). For the HT-34 calculations, no gaps were assumed between coatings, which could mean the actual kernel temperatures were higher. The kernel temperatures were $30^{\circ}$ to $50^{\circ} \mathrm{C}$ higher than the surface temperatures for the low-temperature samples and $50^{\circ}$ to $70^{\circ} \mathrm{C}$ higher for the high-temperature samples. The average kernel temperature for the two magazines reached $1410^{\circ}$ and $1620^{\circ} \mathrm{C}$ during the last cycle. 


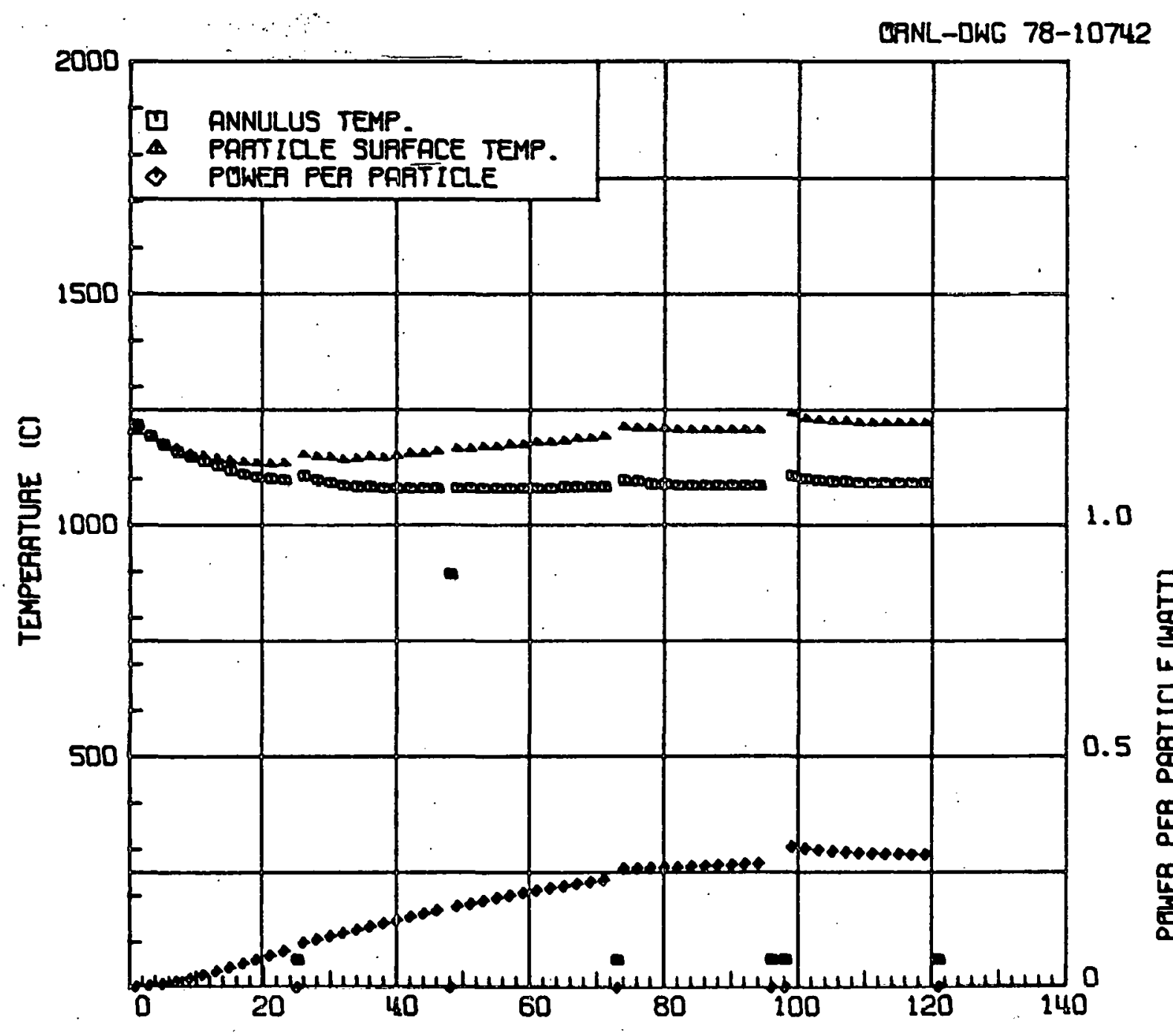

TIME FRUM BEGINNING OF IRRADIATIEN IDAYSI

HT-34 PART ICLE HOLDER 2 BATCH 20-0161-001 862.0 MICRON PARTICLE DIAMETER

Fig. 4-1. Results of ORNL thermal analysis (sheet 1 of 16) 


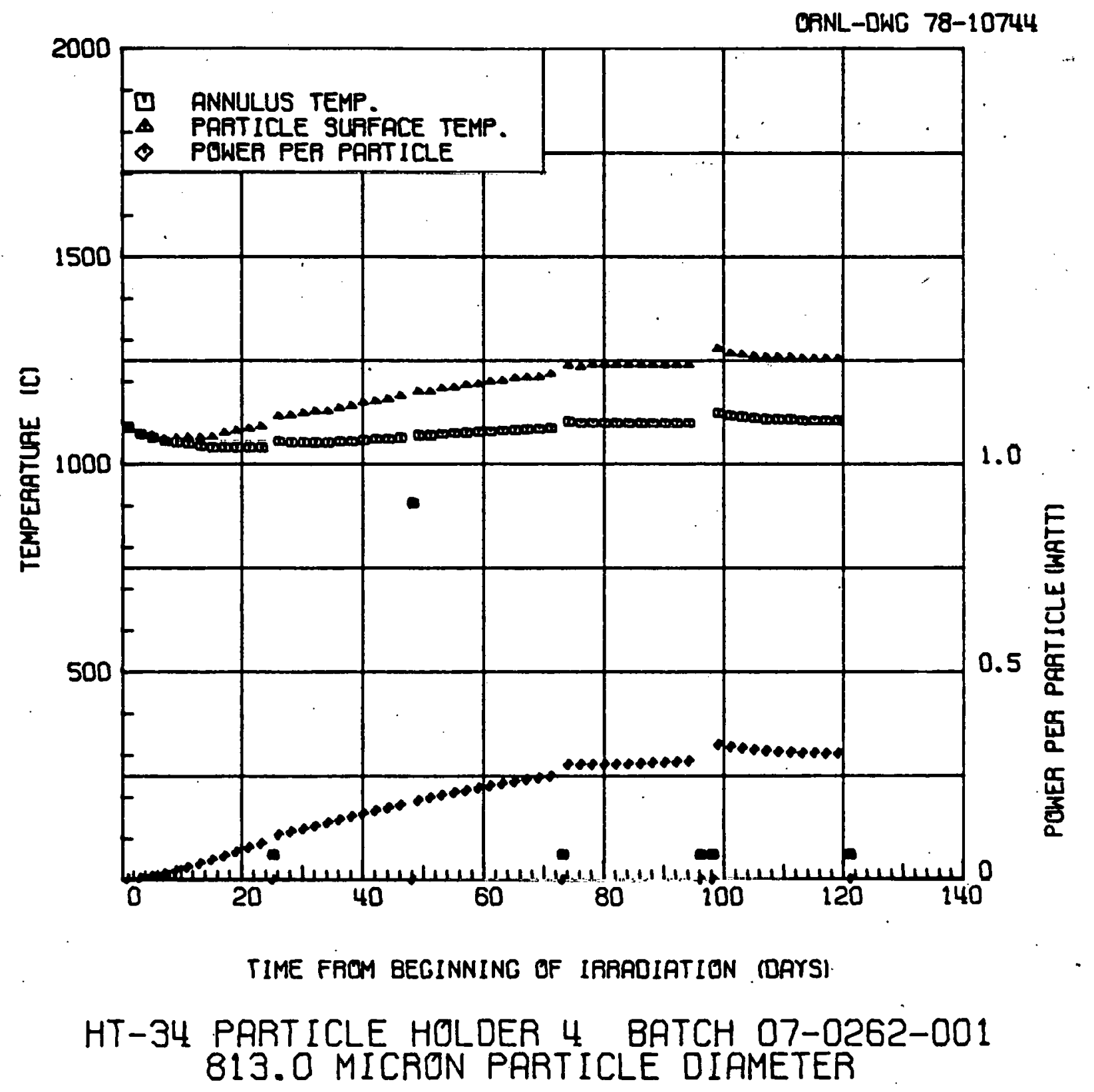

Fig. 4-1. Results of ORNL thermal analysis (sheet 2 of 16) 


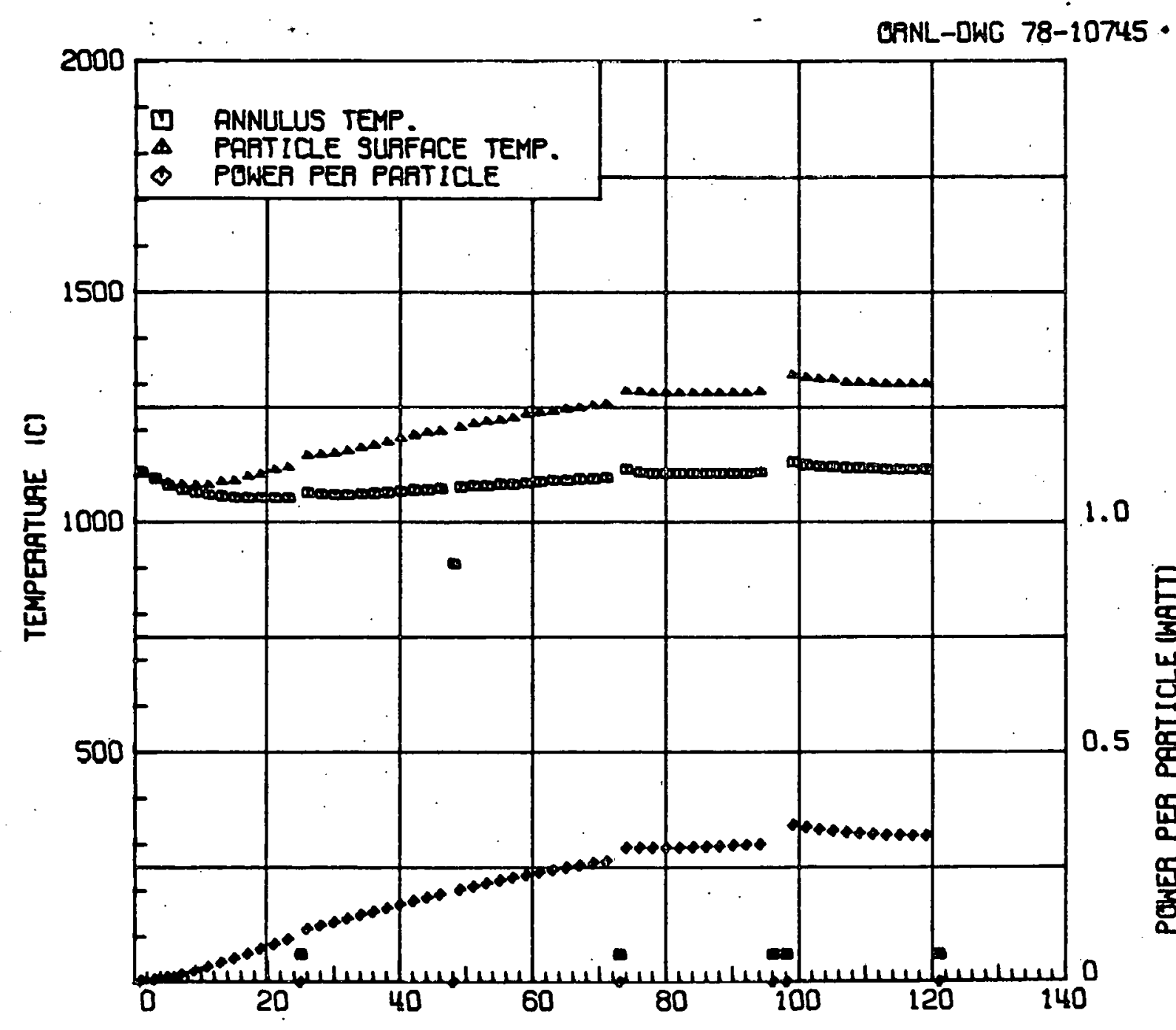

TIME FREM BECINNINC OF IRRADIATION IDAYSI

HT-34 PART ICLE HOLDER 5 BATCH 1.4-0261-001 1 729.0 MICRON PARTICLE DIRMETER

Fig. 4-1. Results of ORNL thermal analysis (sheet 3 of 16) 


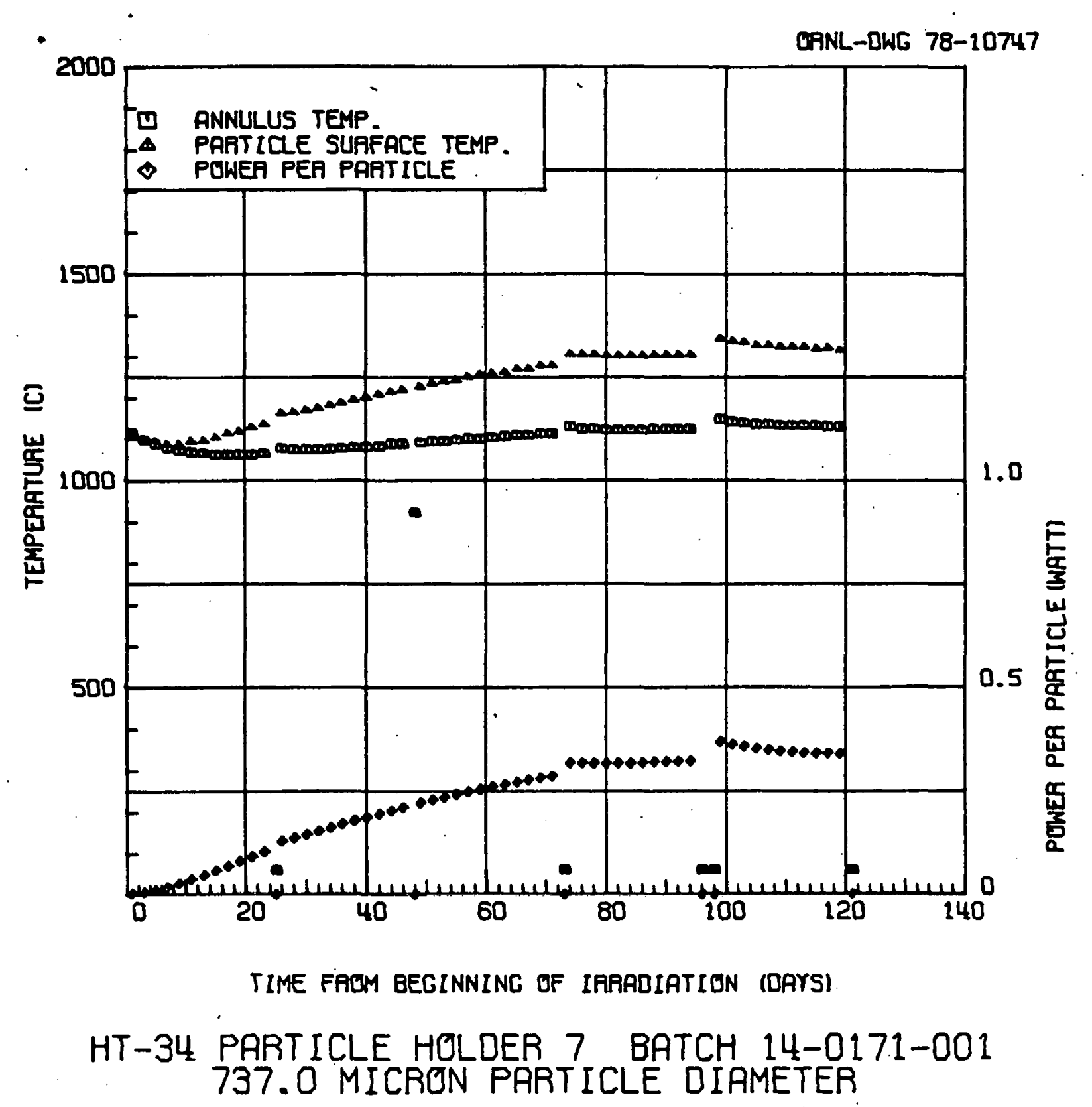

Fig. 4-1. Results of ORNL thermal analysis (sheet 4 of 16) 


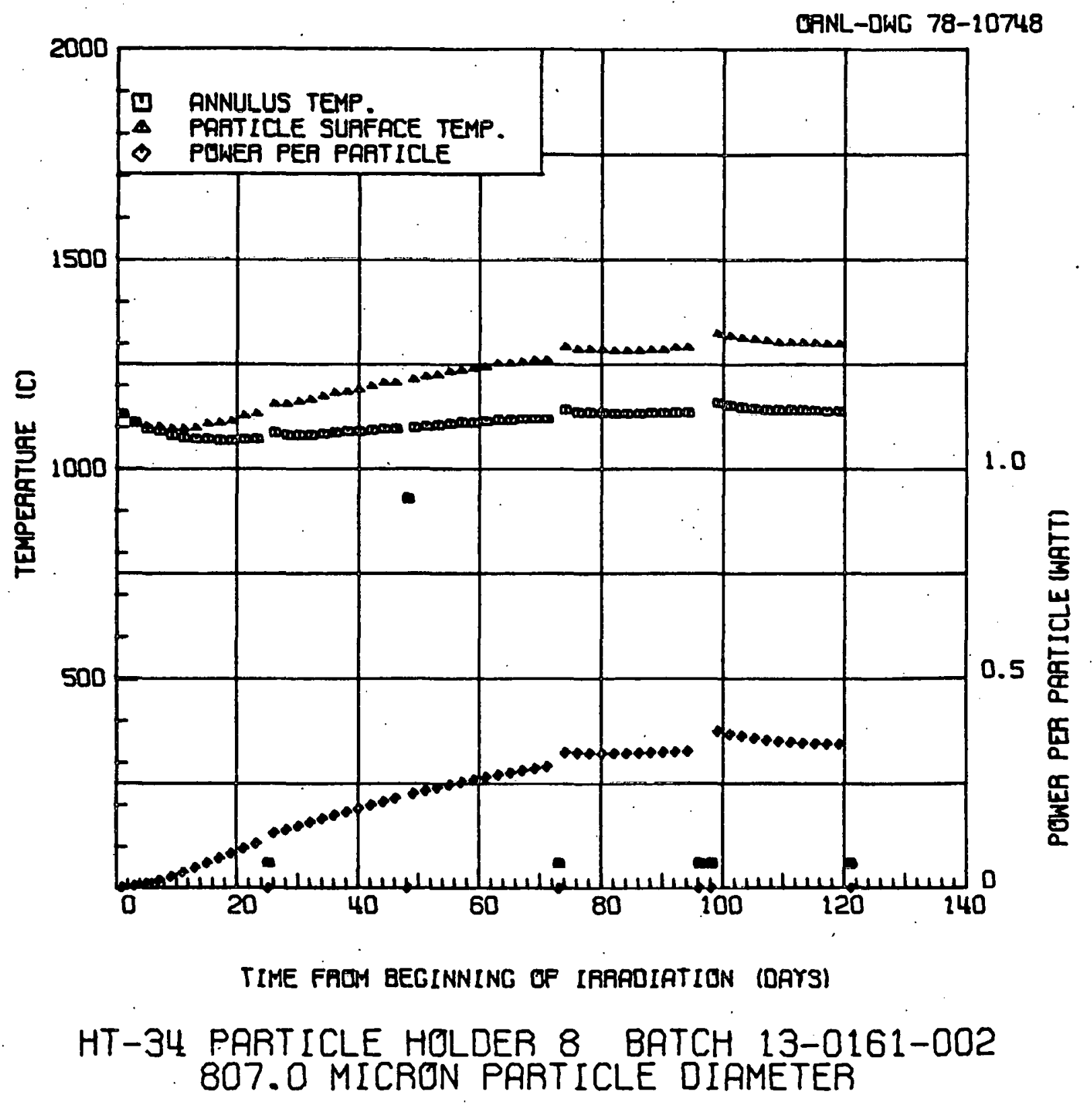

Fig. 4-1. Results of ORNL thermal analysis (sheet 5 of 16) 


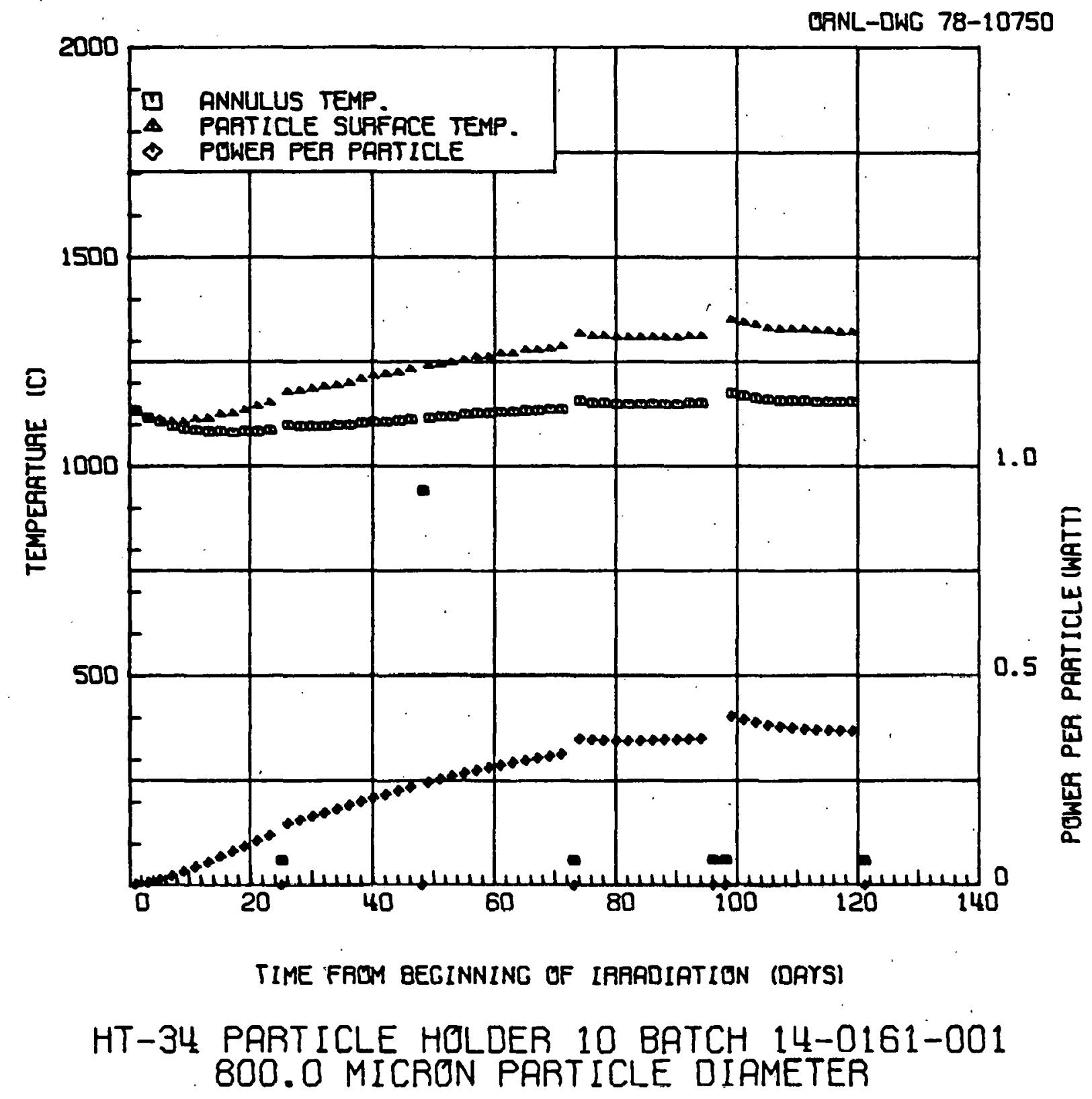

Fig. 4-1. Results of ORNL thermal analysis (sheet 6 of 16) 


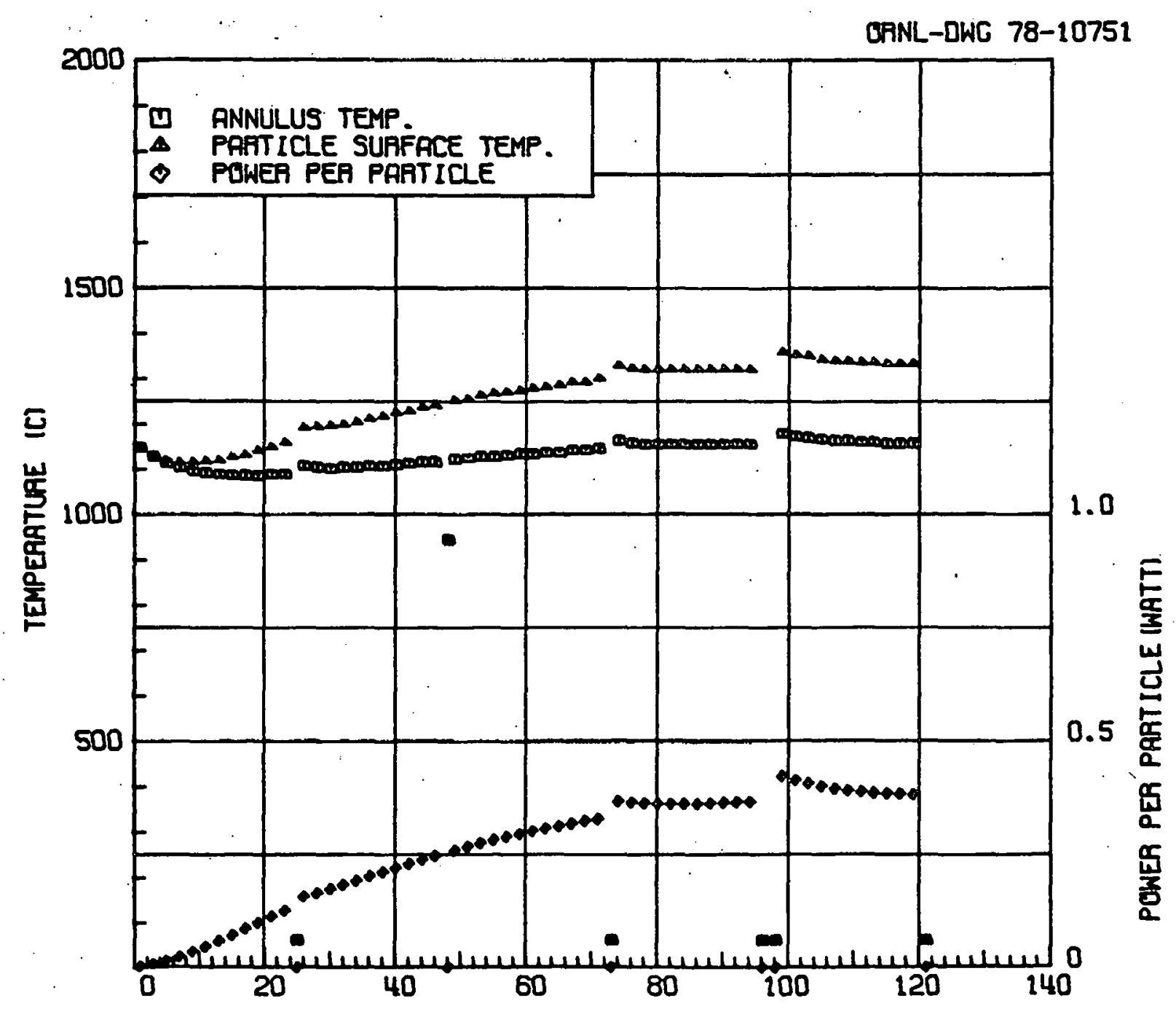

TIME FAOM BELINNLNG OF LARADIATION (DAYSI HT-34 PARTICLE HOLDER 11 BATCH 15-0161-001 800.0 MICRON PART ICLE DIAMETER

Fig. 4-1. Results of ORNL thermal analysis (sheet 7 of 16) 


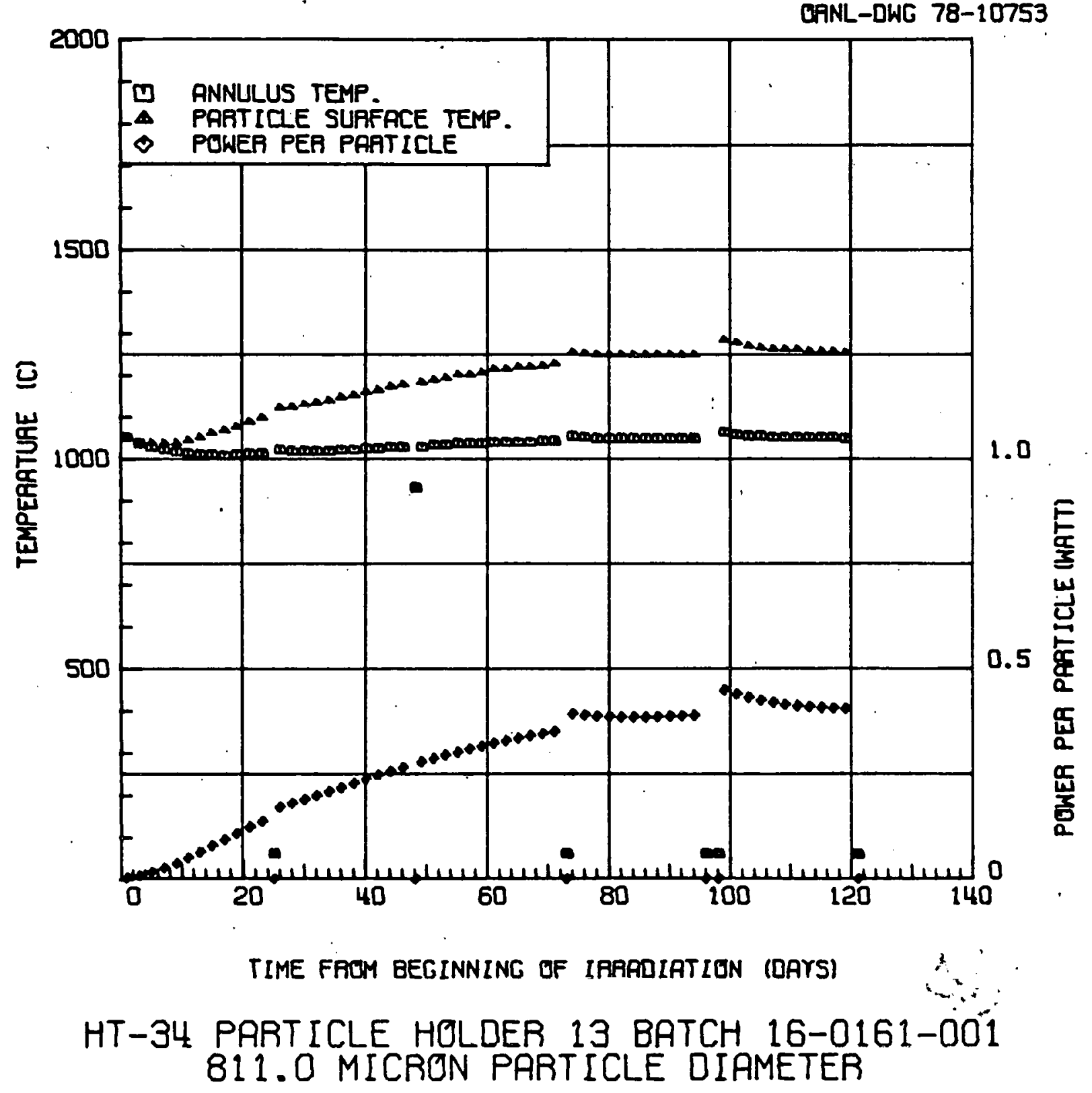

Fig. 4-1. Results of ORNL thermal analysis (sheet 8 of 16) 


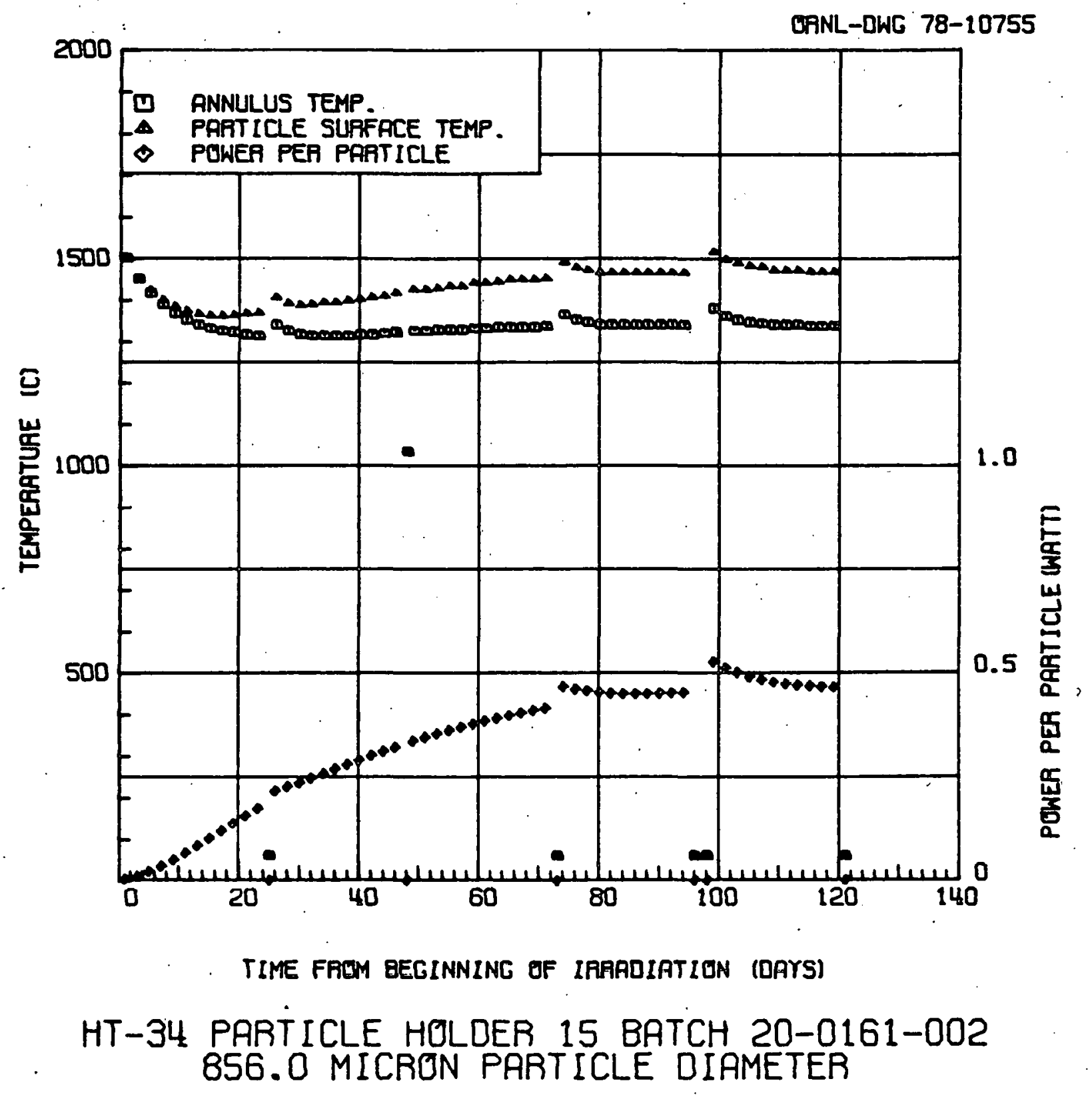

Fig. 4-1. Results of ORNL thermal analysis (sheet 9 of 16) 


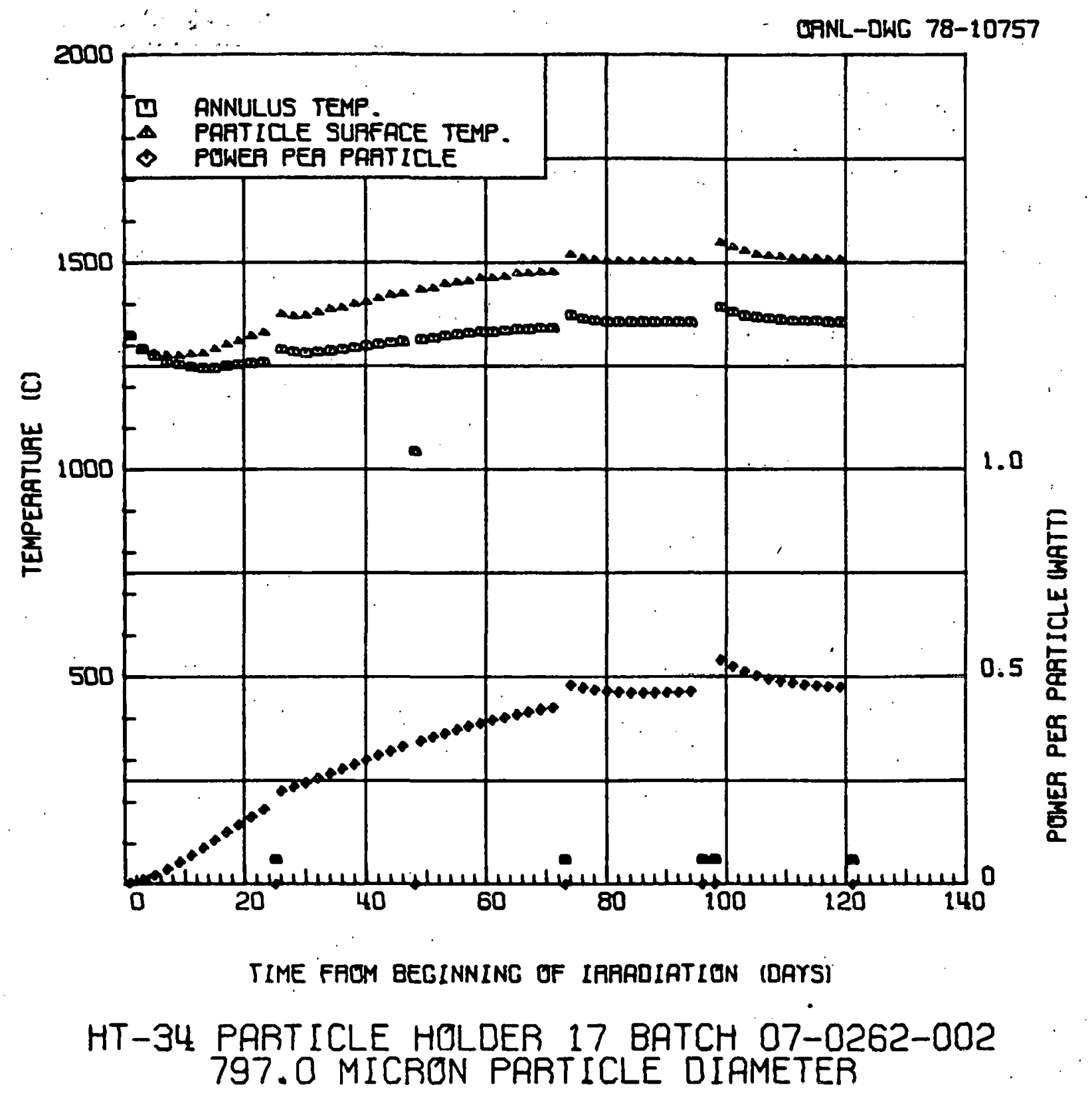

Fig. 4-1. Results of ORNL thermal analysis (sheet 10 of 16) 


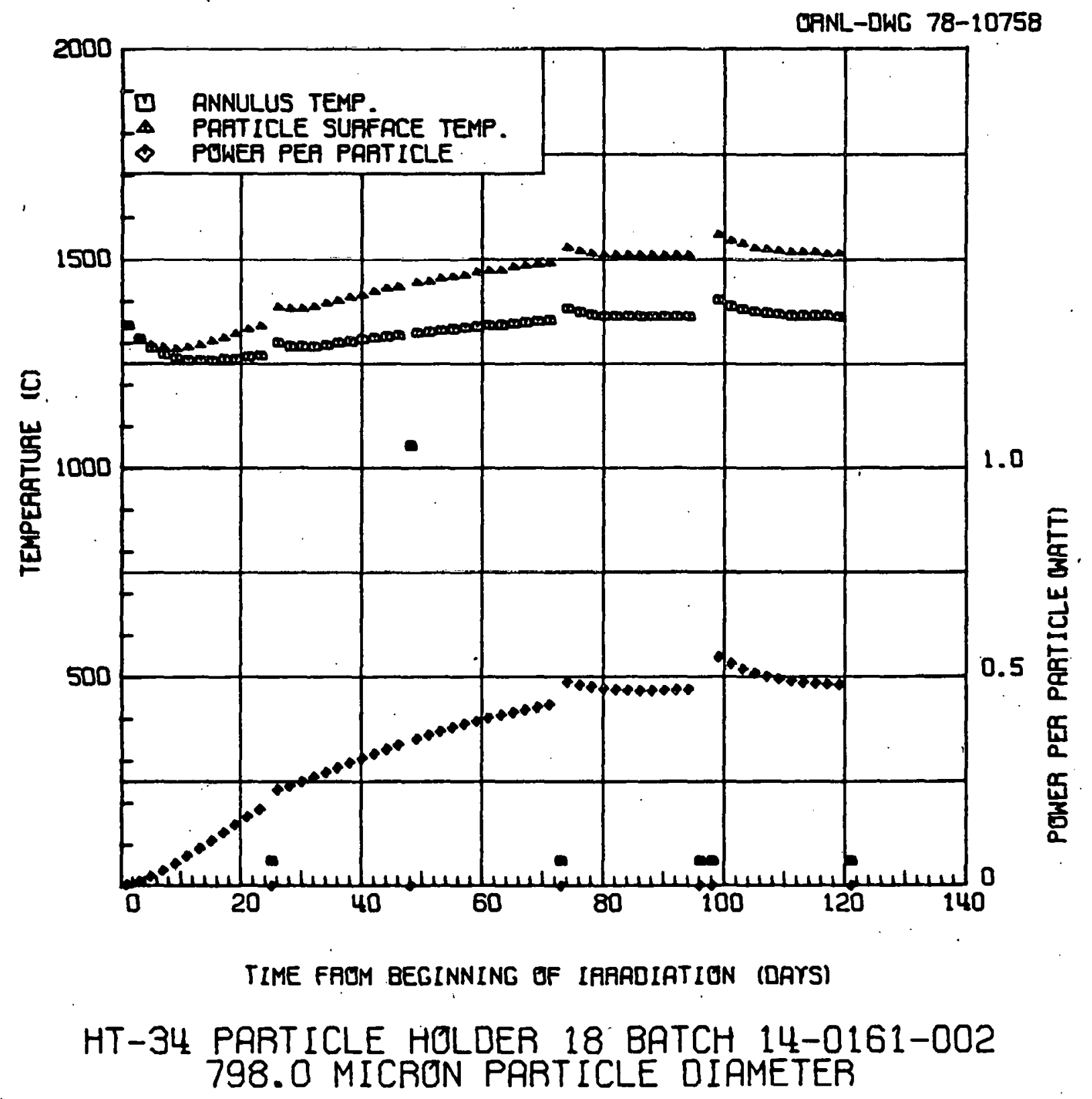

Fig. 4-1. Results of ORNL thermal analysis (sheet 11 of 16) 


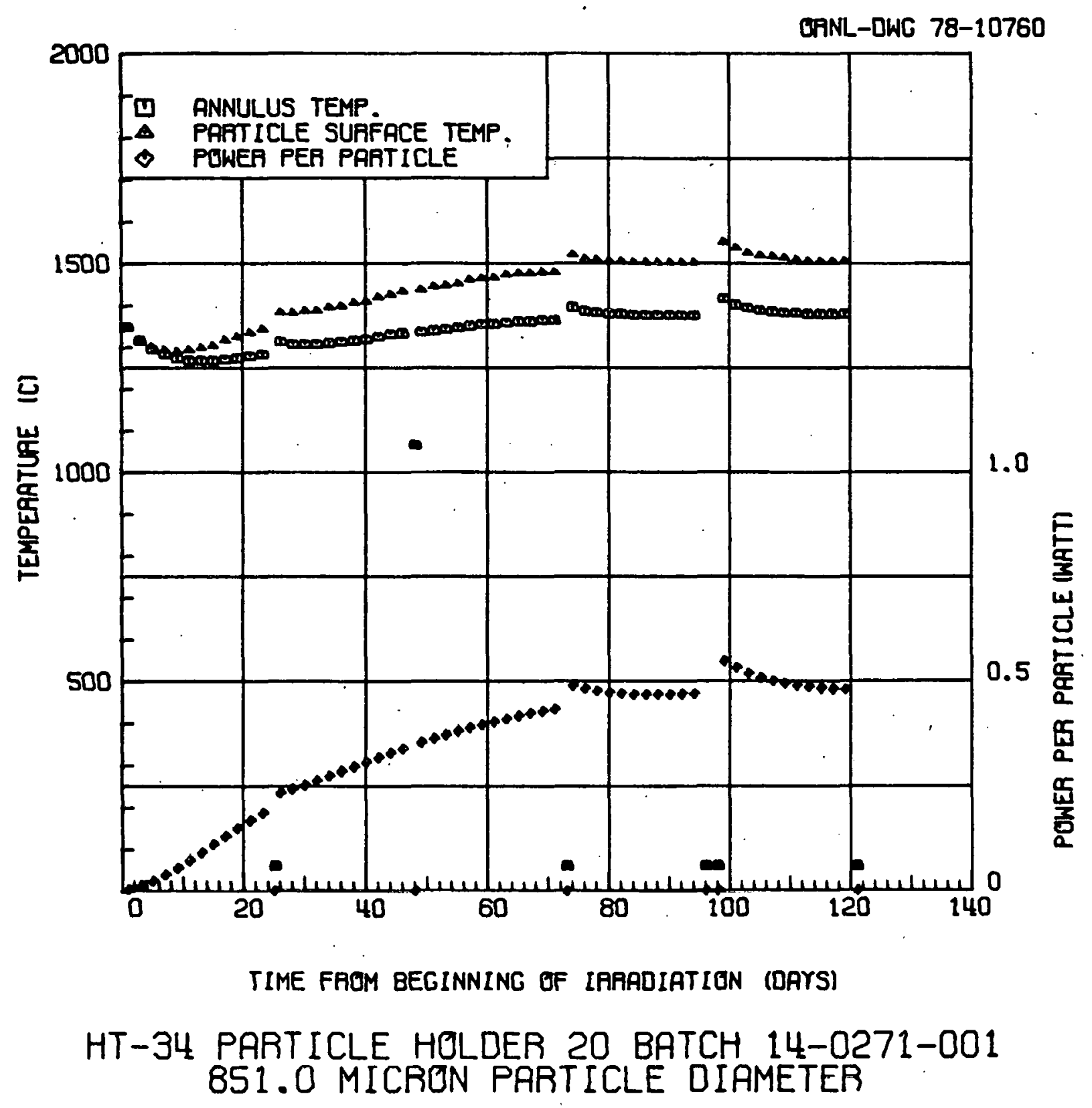

Fig. 4-1. Results of ORNL thermal analysis (sheet 12 of 16) 


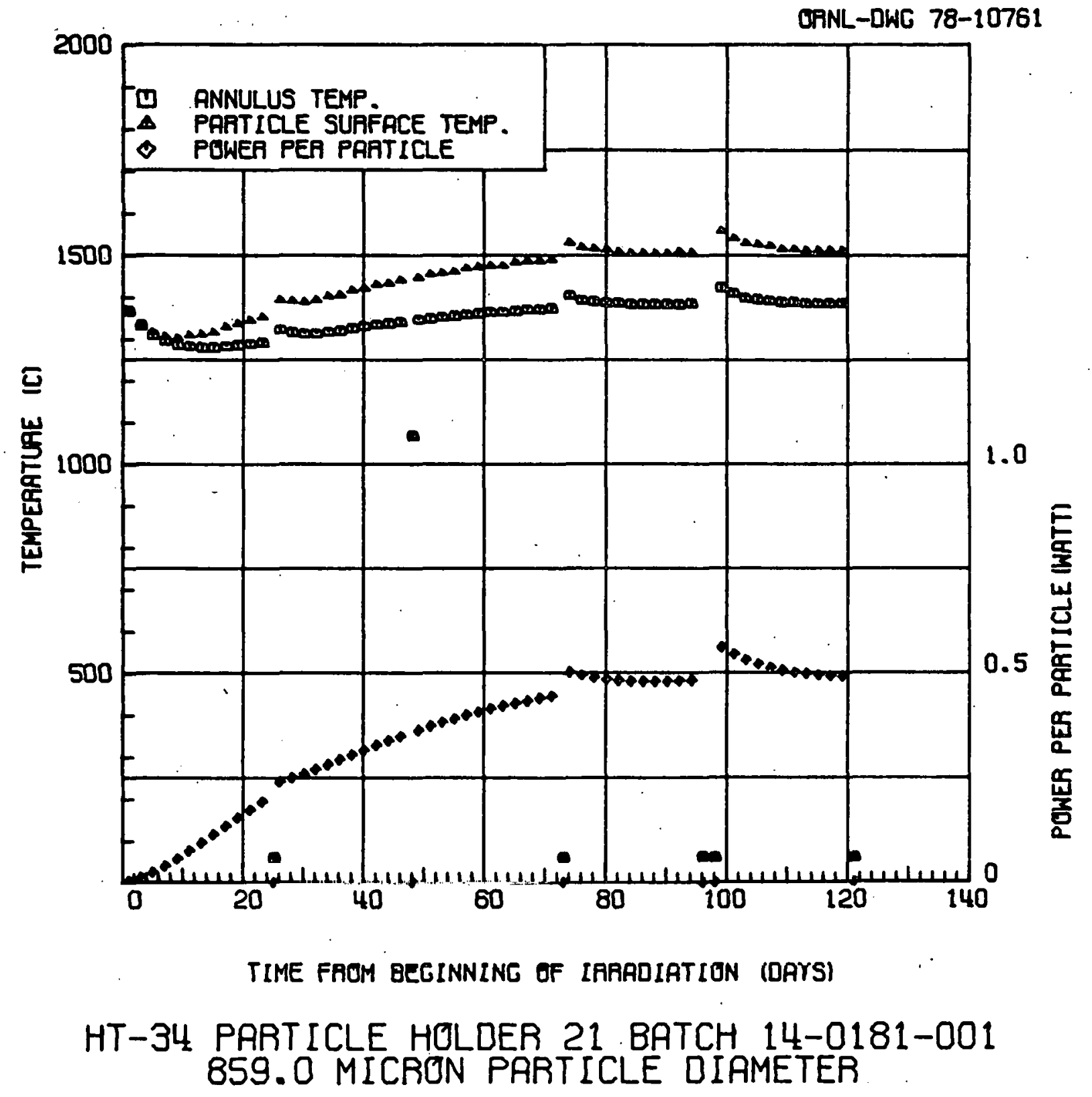

Fig. 4-1. Results of ORNL thermal analysis (sheet 13 of 16) 
GANL-DWC 78-10763

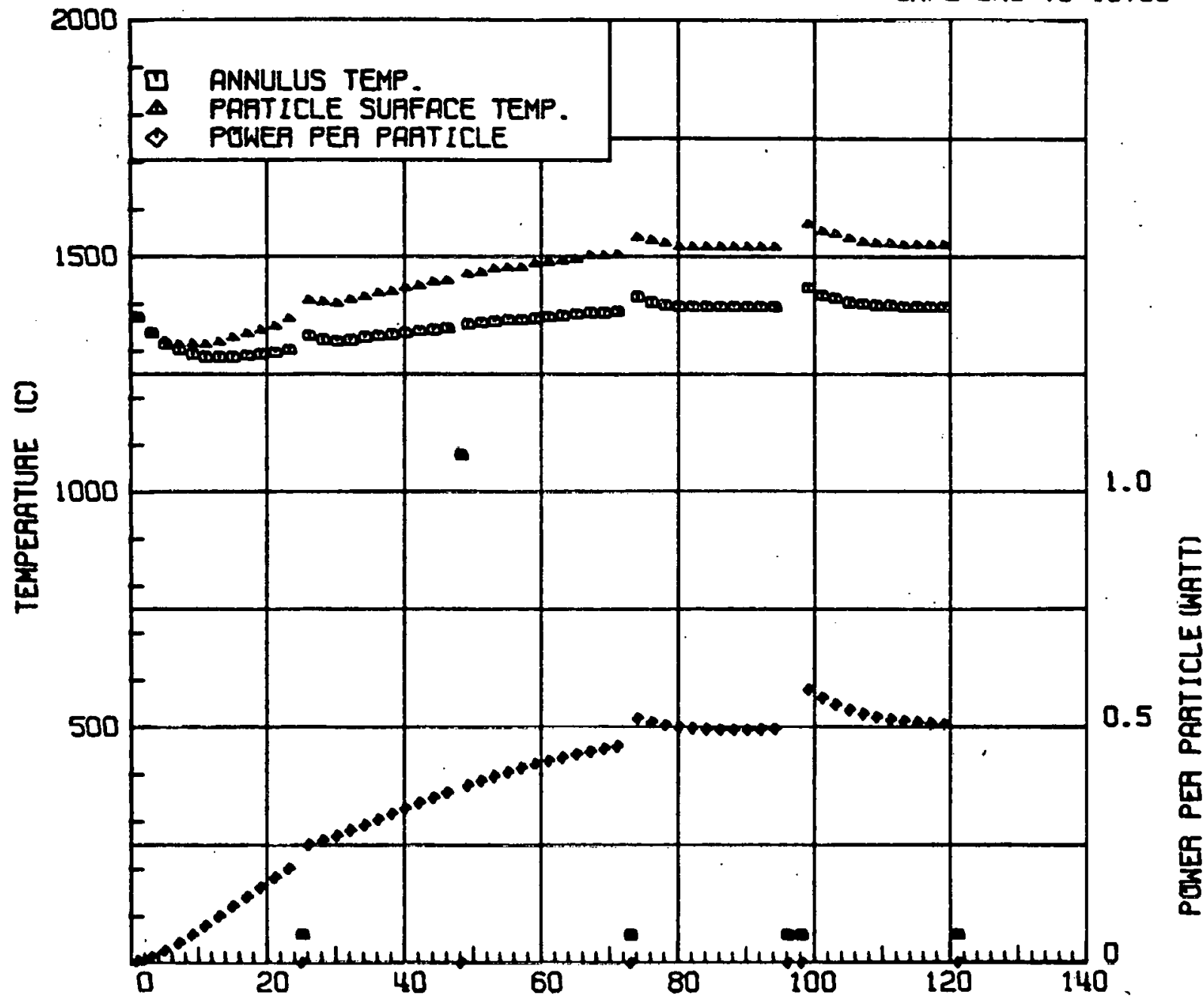

TIME FAOM BECINNING OF IRARDIATION (DAYS)

HT-34 PARTICLE HOLDER 23 BATCH 17-0161-001 853. 0 MICRON PART ICLE DIAMETER

Fig. 4-1. Resuits of ORNL thermal analysis (sheet 14 of 16) 


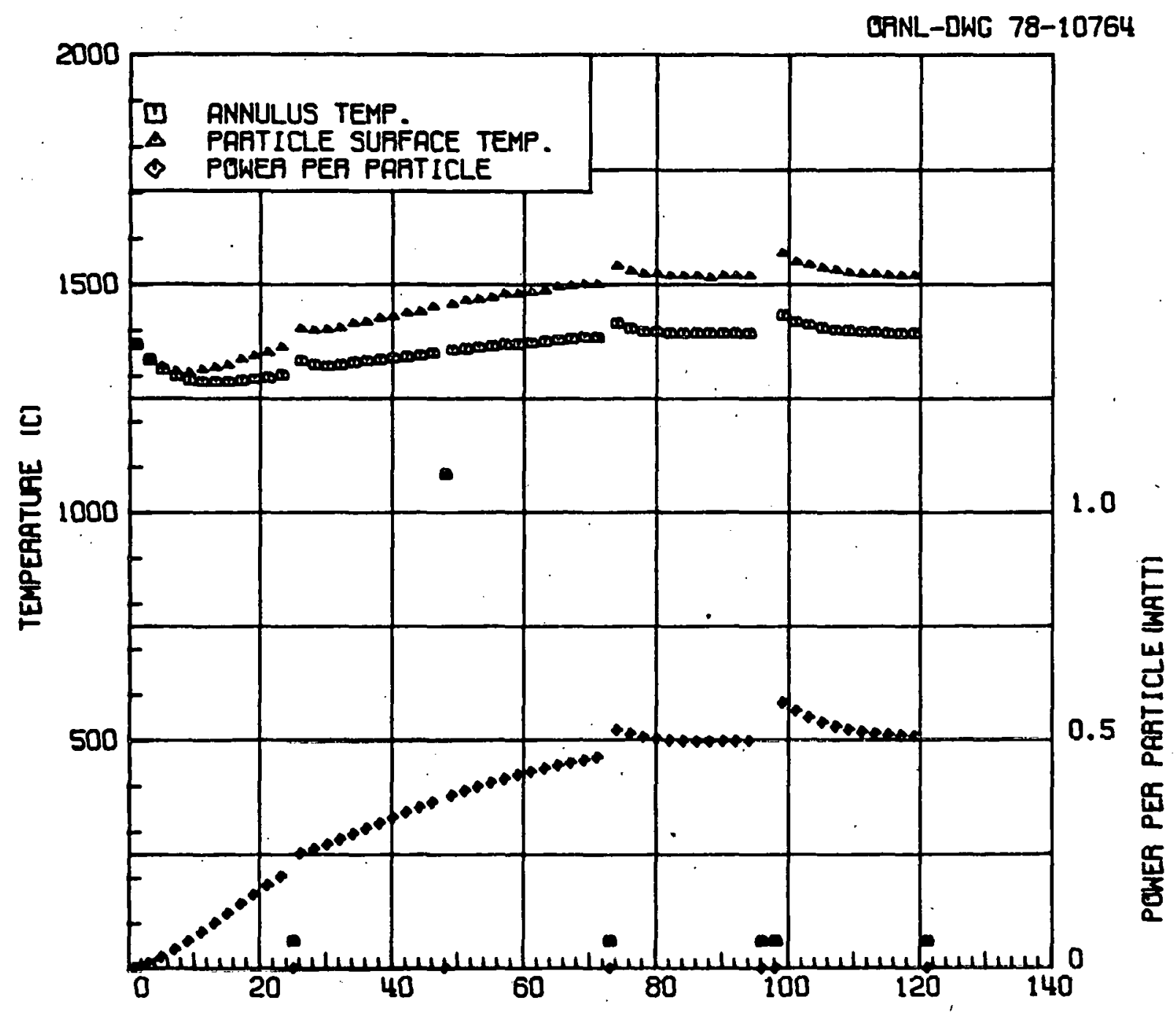

TIME FRIM REGINNING OF IARADIATION (DAYSI

HT-34 PARTICLE HOLDER 24 BATCH 15-0171-001 866.0 MICRON PARTICLE DIAMETER

Fig. 4-1. Results of ORNL thermal analysis (sheet 15 of 16) 
GANL-DWG 78-10766

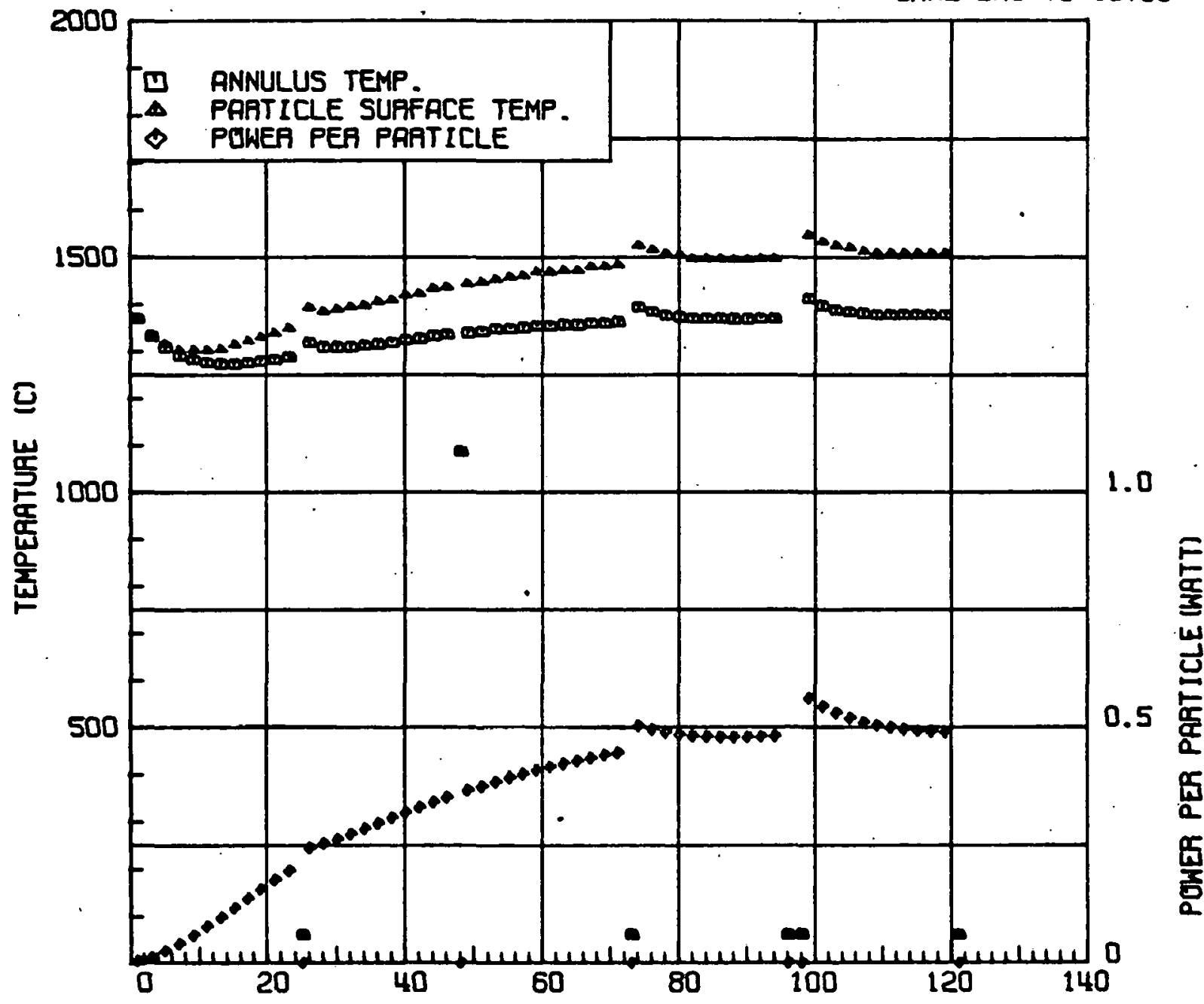

TIME FRUM BEGINNING OF IARADIATION (DAYS)

HT-34 PARTICLE HOLDER 26 BATCH 13-0171-001 849.0 MICRON PARTICLE DIAMETER

Fig. 4-1. Results of ORNL thermal analysis (sheet 16 of 16) 
TABLE 4-1

REACTOR OPERATING HISTORY $(a)$

\begin{tabular}{|c|c|c|c|c|c|c|}
\hline \multirow{3}{*}{$\begin{array}{l}\text { HFIR } \\
\text { Cycle }\end{array}$} & \multicolumn{4}{|c|}{ Cycle Dates } & \multirow{2}{*}{\multicolumn{2}{|c|}{$\begin{array}{c}\text { Irradiation Time } \\
(\mathrm{hr})\end{array}$}} \\
\hline & \multicolumn{2}{|c|}{ Begin } & \multicolumn{2}{|c|}{ End } & & \\
\hline & Day & Time & Day. & Time & Cycle & Accumulated \\
\hline 150 & $07 / 27 / 77$ & $19: 00$ & $08 / 17 / 77$ & $16: 00$ & 549 & 549 \\
\hline 151 & $08 / 19 / 77$ & $04: 51$ & $09 / 11 / 77$ & $02: 27$ & 547 & 1096 \\
\hline 152 & $09 / 11 / 77$ & $18: 39$ & $10 / 04 / 77$ & $11: 35$ & 545 & 1641 \\
\hline 153 & $10 / 06 / 77$ & $19: 09$ & $10 / 29 / 77$ & $04: 00$ & 538 & 2179 \\
\hline $154^{(c)}$ & $10 / 31 / 77$ & $17: 30$ & $11 / 23 / 77$ & $04: 00$ & 507 & 2686 \\
\hline
\end{tabular}
(a) Ref. 14
(b) Time at $100 \mathrm{MW}$
(c) The reactor was shutdown for $55.3 \mathrm{hrs}$ (from 05:00 11/07/77
to $12: 0011 / 09 / 77$ ) 
TABLE 4-2

NEUTRON FLUENCES AND BURNUPS OF SAMPLES

\begin{tabular}{|c|c|c|c|c|}
\hline \multirow{3}{*}{$\begin{array}{l}\text { Capsule } \\
\text { Position }\end{array}$} & \multirow{3}{*}{$\begin{array}{l}\text { Fast } \\
\text { Fluence (a) } \\
\left(\mathrm{x} 10^{25} \mathrm{n} / \mathrm{m}^{2}\right) \\
(\mathrm{E}>29 \\
\mathrm{fJ}) \text { HTGR }\end{array}$} & \multicolumn{3}{|c|}{ Burnup } \\
\hline & & \multicolumn{2}{|c|}{$\%$ FIMA } & \multirow{2}{*}{$\operatorname{Difference}_{(\%)}^{(c)}$} \\
\hline & & ORNL $^{(a)}$ & $\mathrm{GA}^{(\mathrm{b})}$ & \\
\hline \multicolumn{5}{|c|}{ Low Temperature Magazine } \\
\hline 2 & 5.1 & $\hat{5.6}$ & 5.1 & 9.8 \\
\hline 4 & 5.8 & 6.4 & 5.7 & 12.2 \\
\hline 5 & 6.1 & 6.7 & 6.0 & 11.7 \\
\hline$\hat{7}$ & 6.7 & 7.3 & 6.7 & 10.4 \\
\hline 8 & 7.0 & 7.6 & 7.0 & 8.6 \\
\hline 10 & 7.5 & 8.3 & 7.6 & 9.2 \\
\hline 11 & 7.7 & 8.6 & 7.9 & 8.9 \\
\hline 13 & 8.2 & 9.2 & 8.5 & 8.2 \\
\hline
\end{tabular}

\begin{tabular}{l|r|r|r|r}
\hline \multicolumn{5}{c}{ High Temperature Magazine } \\
\hline 15 & 9.2 & 10.7 & 10.5 & 1.9 \\
17 & 9.4 & 11.0 & 10.9 & .9 \\
18 & 9.5 & 11.2 & 11.2 & 0 \\
20 & 9.7 & 11.5 & 11.6 & -0.8 \\
21 & 9.8 & 11.6 & 11.9 & -2.5 \\
23 & 10.0 & 11.9 & 12.2 & -2.5 \\
24 & 10.1 & 12.0 & 12.4 & -3.2 \\
26 & 10.2 & 12.2 & 12.7 & -3.9 \\
\hline
\end{tabular}

(a) Ref. 14

(b) Ref. 15

(c) $[($ ORNL-GA) $\div$ GA $] \times 100$ 
TABLE 4-3

TIME-AVERAGED MAXIMUM SURFACE TEMPERATURES ${ }^{(a)}$ OF SAMPLES

\begin{tabular}{|c|c|c|c|c|c|c|}
\hline \multirow[b]{2}{*}{$\begin{array}{l}\text { Capsule } \\
\text { Position }\end{array}$} & \multicolumn{6}{|c|}{ Time-Averaged Maximum Surface Temperature $\left({ }^{\circ} \mathrm{C}\right)$} \\
\hline & Cycle 1 & Cycle 2 & Cycle 3 & Cycle 4 & Cycle 5 & $\begin{array}{c}\text { Average } \\
\text { of } \\
\text { Cycles }\end{array}$ \\
\hline \multicolumn{7}{|c|}{ Low Temperature Magazine } \\
\hline 2 & 1150 & 1150 & 1180 & 1210 & 1220 & 1180 \\
\hline 4 & 1070 & 1140 & 1200 & 1240 & 1260 & 1180 \\
\hline 5 & 1090 & 1170 & 1240 & 1280 & 1300 & 1210 \\
\hline 7 & 1100 & 1190 & 1260 & 1300 & 1320 & 1230 \\
\hline 8 & 1110 & 1180 & 1240 & 1280 & 1300 & 1220 \\
\hline 10 & 1120 & 1200 & 1270 & 1310 & 1330 & 1240 \\
\hline 11 & 1130 & 1210 & 1280 & 1320 & 1340 & 1250 \\
\hline 13 & 1060 & 1150 & 1210 & 1250 & 1260 & 1180 \\
\hline \multicolumn{7}{|c|}{ High Temperature Magazine } \\
\hline 15 & 1390 & 1400 & 1440 & 1470 & 1480 & 1430 \\
\hline 17 & 1290 & 1400 & 1460 & 1500 & 1520 & 1430 \\
\hline 18 & 1310 & 1400 & 1470 & 1510 & 1520 & 1440 \\
\hline 20 & 1310 & 1400 & 1470 & 1500 & 1500 & 1440 \\
\hline 21 & 1320 & 1410 & 1470 & 1510 & 1520 & 1440 \\
\hline 23 & 1330 & 1420 & 1490 & 1520 & 1530 & 1460 \\
\hline 24 & 1330 & 1420 & 1480 & 1.520 & 1530 & 1450 \\
\hline 26 & 1320 & 1410 & 1470 & 1500 & 1510 & 1400 \\
\hline
\end{tabular}

${ }^{(a)} \operatorname{Ref} .17$. 
TABLE $4-4$

POWER PER PARTICLE OF SAMPLES

\begin{tabular}{|c|c|c|c|c|c|c|}
\hline \multirow[b]{2}{*}{$\begin{array}{l}\text { Capsule } \\
\text { Position }\end{array}$} & \multicolumn{6}{|c|}{ Time-Averaged Power/Particle (Watts) } \\
\hline & Cycle 1 & Cycle 2 & Cycle 3 & Cycle 4 & Cycle 5 & $\begin{array}{c}\text { Average } \\
\text { for } \\
\text { Capsule }\end{array}$ \\
\hline 2 & .03 & .14 & .21 & .26 & .29 & .18 \\
\hline 4 & .04 & .15 & .23 & .28 & .31 & .20 \\
\hline 5 & .04 & .16 & .24 & .29 & .32 & .21 \\
\hline 7 & .05 & .17 & .26 & .32 & .35 & .23 \\
\hline 8 & .05 & .18 & .26 & .32 & .35 & .23 \\
\hline 10 & .05 & .19 & .29 & .34 & .38 & .25 \\
\hline 11 & .06 & .20 & .30 & .36 & .39 & .26 \\
\hline 13 & .06 & .22 & .32 & .38 & .42 & .28 \\
\hline 15 & .08 & .27 & .38 & .45 & .48 & .33 \\
\hline 17 & .08 & .28 & .39 & .42 & .49 & .33 \\
\hline 18 & .08 & .29 & .40 & .47 & .50 & .34 \\
\hline 20 & .09 & .29 & .40 & .47 & .50 & .35 \\
\hline 21 & .09 & .30 & .41 & .48 & .51 & .35 \\
\hline 23 & .09 & .31 & .43 & .50 & .53 & .37 \\
\hline 24 & .09 & .31 &. .43 & .50 & .53 & .37 \\
\hline 26 & .09 & .30 & .42 & .48 & .51 & .36 \\
\hline
\end{tabular}


TABLE $4-5$

TIME-AVERAGED KERNEL TEMPERATURES OF SAMPLES

\begin{tabular}{|c|c|c|c|c|c|c|}
\hline \multirow[b]{2}{*}{$\begin{array}{l}\text { Capsule } \\
\text { Position }\end{array}$} & \multicolumn{6}{|c|}{ Time-Volume-Averaged Kernel Temperatures $\left({ }^{\circ} \mathrm{C}\right)$} \\
\hline & Cycle 1 & Cycle 2 & Cycle 3 & Cycle 4 & Cycle 5 & $\begin{array}{c}\text { Average } \\
\text { for } \\
\text { Capsule }\end{array}$ \\
\hline 2 & 1160 & 1170 & 1220 & 1260 & 1270 & 1210 \\
\hline 4 & 1080 & 1160 & 1240 & 1290 & 1310 & 1210 \\
\hline 5 & 1100 & 1190 & 1270 & 1320 & 1350 & 1240 \\
\hline 7 & 1110 & 1220 & 1300 & 1350 & 1370 & 1270 \\
\hline 8 & 1120 & 1210 & 1280 & 1330 & 1360 & 1260 \\
\hline 10 & 1130 & 1230 & 1320 & 1370 & 1390 & 1290 \\
\hline 11 & 1140 & 1240 & 1330 & 1380 & 1410 & 1300 \\
\hline 13 & 1070 & 1190 & 1260 & 1310 & 1330 & 1230 \\
\hline 15 & 1400 & 1450 & 1510 & 1550 & 1560 & 1490 \\
\hline 17 & 1300 & 1450 & 1530 & 1570 & 1600 & 1490 \\
\hline 18 & 1320 & 1450 & 1540 & 1590 & 1600 & 1500 \\
\hline 20 & 1330 & 1450 & 1540 & 1580 & 1590 & 1500 \\
\hline 21 & 1340 & 1460 & 1540 & 1590 & 1610 & 1510 \\
\hline 23 & 1350 & 1470 & 1560 & 1610 & 1620 & 1520 \\
\hline 24 & $1350^{\circ}$ & 1470 & 1560 & 1610 & 1620 & 1520 \\
\hline 26 & 1340 & 1460 & 1540 & 1580 & 1600 & 1500 \\
\hline
\end{tabular}


Blank

$4-24$ 


\section{RESULTS OF POSTIRRADIATION EXAMINATIONS}

The irradiation capsule was disassembled at ORNL. The ends of the capsule containment were cut off and the four graphite magazines were removed. All GA sample holders were pushed out of the top two magazines. The particles from each graphite holder were removed, visually, examined, and photographed in-cell. Selected fuel particles were gamma-counted at ORNL. The samples were then shipped to GA for completion of the postirradiation characterization, which included visual examinations, fission gas release measurements, and metallographic examinations.

\subsection{VISUAL EXAMINATION}

The visual examination was performed at ORNL using an in-cell stereomicroscope with a magnification range of $6 \mathrm{x}$ to $27 \mathrm{x}$. Each sample was examined for particle failure immediately after the particles were.unloaded from the graphite holder. The results are reported in Table 5-1 along with the irradiation conditions, $95 \%$ confidence limits, and the predicted particle failure.

The visual examination of the $1200^{\circ} \mathrm{C}$ magazine indicated that no pressure vessel failure (both OPyC and SiC coatings cracked) occurred in any of the samples. The OPyC coating failure was zero for five samples, $1.8 \%$ for two, and $8.9 \%$ for one, and was apparently caused by fast neutron damage, as shown in Fig. 5-1. A typical sample exhibiting zero pressure vessel and OPyC coating failure is shown in Fig. 5-2.

The visual examination of the $1450^{\circ} \mathrm{C}$ magazine samples showed that the OPyC plus SiC coating failure ranged from 6.2 to $>80 \%$. The $\mathrm{ThO}_{2}$ kernels of the failed particles fragmented and converted to carbide by the end of the irradiation. The OPYC coating failure was the same as the pressure vessel failure. An example of the failure is shown in Fig. 5-3. 
The two end plugs containing the burned-back TRISO inert particles (batch 6351-040-0100) were examined at GA. The end plugs were unloaded at ORNL, and the particles were shipped to GA.

End plug 14-1 was irradiated at $1200^{\circ} \mathrm{C}$. Totals of $36 \mathrm{Si}$-doped BISO particles (batch 7032-149) and 14 TRISO particles were examined. No coating failures were observed in either batch. The SiC coating of the burned-back inerts was visibly in good condition. Twenty BISO and 11 TRISO particles were lost during unloading at ORNL.

End plug $15-2$ was irradiated at $\sim 1500^{\circ} \mathrm{C}$. Forty-five BISO particles and one TRISO particle were counted and all particles were intact. The SiC coating of the one TRISO particle was shiny and appeared to be in good condition. Thirty-five BISO and 24 TRISO particles were missing from the sample.

The burned-back TRISO inert particles were placed in capsule HT-34 to determine if the exposed SiC coating volatilized during irradiation. The visual examination indicated that volatilization did not occur, because the SiC surface still appeared shiny through the microscope at $27 x$. No other analyses were performed on these particles.

\subsection{METALLOGRAPHIC EXAMINATION}

S1x particle samples were examined metallographically to evaluate the microstructure of the coatings and kernels after irradiation. Approximately 15 particles from each specimen were mounted. The mounts were ground, reimpregnated with mounting resin to prevent kernel pullout, reground, and finally polished. The polished sections were examined under bright field illumination and polarized light with a Lietz metallograph. The results of the examination are presented in Table 5-2 and given in terms of number of observations rather than percentages, because the sample sizes were very small. The data are useful, for a qualitative evaluation. 
The integrity of the sample particles was indicated by the metallographic examination.: Zero $\mathrm{SiC}$ and OPyC coating failure was observed in the polished sections of four $1200^{\circ} \mathrm{C}$ magazine samples. This agrees with the visual results. The two $1450^{\circ} \mathrm{C}$ samples exhibited high SiC coating failure but low OPyC coating failure. These data are also consistent with the visual results, because SiC failure would not be detected if the OPyC were still intact. The anisotropy of the OPyC and IPyC coatings, which was observed under polarized light, increased slightly during irradiation, as shown in Fig. 5-4. The IPyC coating cracked and/or debonded in the majority of particles examined. Figure 5-4 also indlcates that redeposition of the buffer coating was observed. The metallographic examination showed that the particles with the 27- $\mu \mathrm{m}$ buffer (6252-14-0261-001) performed well. An example is given in Fig. 5-5.

The shape and microstructure of the kernels changed with temperature and burnup. At a temperature of $1240^{\circ} \mathrm{C}$ and a burnup of. $6.0 \%$ FIMA the kernels were round, and the grain boundaries and a flne porosity were visible, as shown in Fig. 5-5. The kernels irradiated at $1280^{\circ} \mathrm{C}$ to a burnup of $7.6 \%$ FIMA were similar, but no grain boundaries were visible (Fig. 5-4). A white metallic phase approximately 1 to $2 \mu \mathrm{m}$ in size appeared throughout the kernels. At temperatures of $1490^{\circ} \mathrm{C}$ and a burnup of $10.5 \%$ to $10.9 \%$ FIMA, the kernels elongated, the microstructure showed larger pores, and the amount of the white metallic phase increased (Fig. 5-6).

Different types of chemical attack of the SiC coating were observed in both the $1200^{\circ}$ and $1450^{\circ} \mathrm{C}$ samples. The metallographic evaluation showed that two kinds of chemical reactions occurred in three of the four samples irradiated at $1180^{\circ}$ to $1240^{\circ} \mathrm{C}$. The SiC coating of the majority of these three samples exhibited $\leq 2-\mu \mathrm{m}$ pores distributed around the entire SiC coating. The pores were observed across the SiC coating thickness, but were concentrated near the inner surface of the SiC. The amount of porosity varied from particle to particle. Figure 5-7 gives examples of this phenomenon. The other type of attack in the three $1200^{\circ} \mathrm{C}$ specimens was a localized reaction at the inner surfacc of the SiC coating, as shown in Fig. 5-8. 
In five out of 45 particles examined, there was a penetration of 9 to $18 \mu \mathrm{m}$ of the SiC coating (nominal thickness was $35 \mu \mathrm{m}$ ). A white metallic phase was observed in the front of the reaction zone in one of the five areas (see Fig. 5-8d).

The two $1450^{\circ} \mathrm{C}$ specimens also showed localized attack of the SiC coating, but the appearance of the reaction was different from that in the $1200^{\circ} \mathrm{C}$ samples. In some instances, the corrosion was completely through the SiC coating in a localized area. The reaction zone was always on the hot side of the particle, because the attack was opposite the advancing face of the migrated kernel. No metallic phases were observed at the reaction zone. , In some cases there was a buildup of white phase, which was bonded to the SiC coating near the reaction zone. It had the appearance of being SiC. Photomicrographs of the $1450^{\circ} \mathrm{C}$ SiC attack are presented in Fig. 5-6.

An unusual SiC coating fracture also occurred in the three $1200^{\circ} \mathrm{C}$ samples that had SiC attack. Figure 5-9 gives an example of the cracking. The fractures began at the inner surface of the SiC and propagated tangentially along the SiC at roughly $5 \mu \mathrm{m}$ from the inner surface. The longest crack measured $\sim 170 \mu \mathrm{m}$. The fracture pattern was simllar for all the. cracks. The cracks did not cause the SiC coating to fail.

The metallographic examination showed that a significant fraction (5 and 13 out of 15) of the particles that were irradiated to a temperature of $1490^{\circ} \mathrm{C}$ and a burnup of $10.9 \%$ exhibited kernel migration. Rejected graphite was observed on the cool side of kernels. An example of the migration is shown in Fig. 5-10. The maximum migration distance was approximately $60 \mu \mathrm{m}$.

\subsection{GAMMA-RAY SPECTROMETRY ANALYSIS}

Gamma-ray spectrometry was used to determine fission product inventories of the TRISO $\mathrm{ThO}_{2}$ samples. The loss of metallic fission products gave a measure of SiC failure. 
Oak Ridge National Laboratory measured the gamma-ray spectra on selected samples, using the Irradiated-Microsphere Gamma Analyzer (IMGA) (Ref. 22). The IMGA system automatically gamma counted each particle from a sample. The GA samples were counted approximately 310 days after the end of irradiation.

Eight $1200^{\circ} \mathrm{C}$ and four $1450^{\circ} \mathrm{C}$ magazine samples were gamma counted. All or most particles from each sample were analyzed. The visual examination indicated that all particles measured had intact SiC coatings. The results of the IMGA measurements are given in Table 5-3. The measured activity for the isotopes $\mathrm{Zr}-95, \mathrm{Ru}-106, \mathrm{Cs}-134, \mathrm{Cs}-137, \mathrm{Ce}-144$, and $\mathrm{Pa}-233$ are specified for each sample. Also, the predicted quantities were calculated by GA. The GA values were determined by the computer program "Curie" (Ref. 15). Considering all the uncertainties in calculating the fission product inventory, the agreement between the measured and predicted values is fairly good.

In addition, certain fission product ratios were calculated for each particle gamma counted from a sample. The data are summarized in Table 5-4, the distributions of $\mathrm{Cs}-137 / \mathrm{Zr}-95$ ratios are plotted in Fig. 5-11, and the predicted fission product ratios are given in both. The figure shows that particles from four low-temperature and all four high-temperature samples released Cs; this will be discussed in Section 6 .

\subsection{FISSION GAS RELEASE}

Fission gas release data were obtained by neutron activation in the GA TRIGA reactor facility on six $1200^{\circ} \mathrm{C}$ samples. The primary objective of the postirradiation FGR measurements was to evaluate the fuel failure.

The samples were examined under stereomicroscope, and washed with nitric acid to remove possible surface contamination picked up during sample unloading. 
Fission gas release, measurements were made by irradiating the fuel specimens in a graphite tube furnace designed to fit into a fuel element position of the TRIGA reactor core (Ref. 23). The furnace allowed measurements to be made at high temperatures, independent of the power generated in the fuel. The rods were irradiated at appropriate power levels for $0.5 \mathrm{~h}$ to produce approximately $10^{14}$ fissions in each fuel specimen. During irradiation, the released fission gases were swept with helium into a liquidnitrogen-cooled charcoal trap. The trapped gases were assayed for fission product isotopes by gamma-ray spectroscopy (Ref. 24), using a Ge(Li) detector and a 4096-channel analyzer. The fission product isotope birth fractions were calculated using the neutron flux in the TRIGA core and the endof-life (EOL) fissionable material loadings given in Table 5-5. The adjusted EOL fissile fuel loading for each specimen was determined by expressing the sum of the weights of all remaining fissionable material (U233 and $U-235$ ) in terms of $U-235$, producing the equivalent $\mathrm{Kr}-85 \mathrm{~m}$ yield. The $\mathrm{Kr}-85 \mathrm{~m}$ isotope was selected as the reference isotope because it has a short halff-life ( 4.4 hours) and its gamma-ray energy peak can easily be resolved. A reference temperature of $1100^{\circ} \mathrm{C}$ was used for analysis of fuel failure levels. The results of the measurements are presented in Table $5-5$.

The failure fraction of each sample was calculated from the FGR measurement. The fission gas released during TRIGA activation comes primarily from heavy metal contamination outside the particle coatings and from failed coatings. Since the particles were washed in nitric acid, the amount of surface contamination was minimized. Therefore, to determine the coating failure fractions it was assumed that the fission gas released was all due to failed particles (this gives a maximum particle fallure). The fractional release for failed particle $\left(R / b_{f}\right)$ is given by the following equation-(Ref. 25) for failed $\mathrm{ThO}_{2}$ particles:

$$
r / b_{f}=0.0013+0.00186(\% \text { FIMA }) 0.9 .
$$


The failure fraction (F) was determined by the following equation:

$$
\mathrm{F}=\frac{\mathrm{R} / \mathrm{B}_{\mathrm{TRIGA}}}{\mathrm{r} / \mathrm{b}_{\mathrm{f}}}
$$

The number of failed particles per sample was then calculated by multiplying the failure fraction by the total number of particles activated. If the number of failed particles was less than 0.5 of a particle, it was assumed that a particle was not failed and the fission ges released was a result of contamination. The results of the calculations are given in Table 5-5. Four of the samples showed zero failure, while samples 6252-13-0161-001 and 6252-15-0161-001 exhibited one particle failed (1.8\%). This is not a true indication of the number of particles per sample with failed SiC coatings, because the OPyC coating was intact on most particles. An OPyC coating prevents or slows the diffusion of $\mathrm{Kr}-85 \mathrm{~m}$ in a particle with a failed SiC coating. The gamma-counting results to be discussed in Section 6 show that the SiC failure is higher, based on metallic fission product release.

\subsection{ELECTRON MICROPROBE EXAMINATION}

An electron microprobe examination was performed at ORNL on one $1200^{\circ} \mathrm{C}$ and one $1450^{\circ} \mathrm{C}$ sample. The microprobe examination was done on two metallographically polished sections prepared at GA.

Sample 10 was selected from the $1200^{\circ} \mathrm{C}$ samples, because metallic fission products were seen in the localized reaction zone of the SiC coating. Figure 5-12 gives the optical light photograph and the electron microprobe scans of the area examined. Palladium was associated with the reaction zone. Chlorine was detected in between the buffer and IPyC and next to the inner surface of the SiC coating. No $U$, $\mathrm{Th}$, rare earths, or $\mathrm{Ag}$ were detected at the reaction zone. No elements were detectd in the small pores in the SiC coating. 
Sample 17 , which was irradiated at $\sim 1450^{\circ} \mathrm{C}$, was selected because of the corrosion of the SiC coating. Figure 5-13 gives the optical light photograph and electron microprobe scans of the areas examined. The results show that-the $\mathrm{Si}$ was transported to the area adjacent to the reaction zone. No $\mathrm{Cl}$, fission products, $\mathrm{U}$, or $\mathrm{Th}$ were measured at the SiC reaction zone. The large white phase in the middle of the kernel and the white specks scattered around the kernel were $\mathrm{rich}$ in Mo and $\mathrm{Ru}$. Iron and $\mathrm{Cr}$ were detected in the large void of the particle. However, it is believed that they were from the steel-mesh polishing wheel. Chromium and Fe were measured at $<50$ ppm each on the as-coated particle batch. 


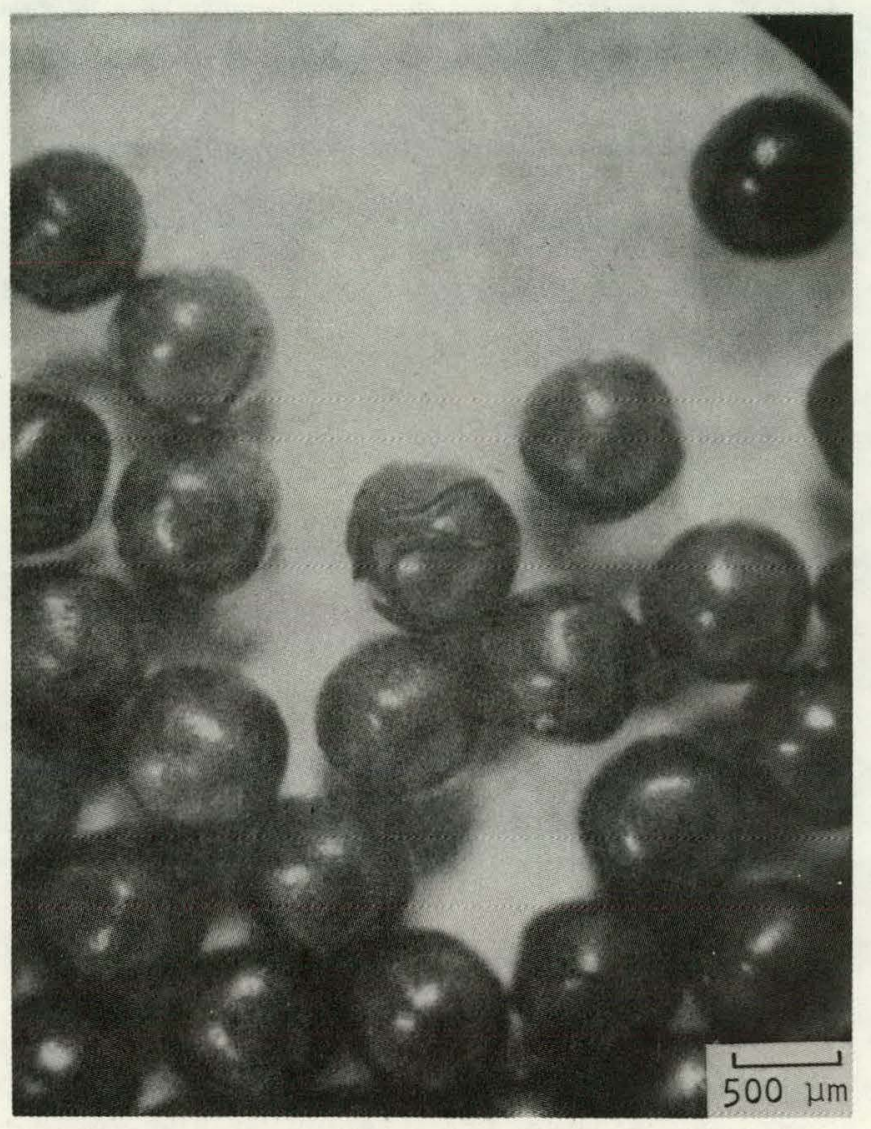

S7909-9

Fig. 5-1. Photomicrograph of a representative example of OPyC coating failure of a particle irradiated in the low temperature magazine; sample 10 had an average temperature of $1240^{\circ} \mathrm{C}$ and a fluence of $7.5 \times 10^{25} \mathrm{n} / \mathrm{m}^{2}(\mathrm{E}>29 \mathrm{fJ})_{\mathrm{HTGR}}$. 


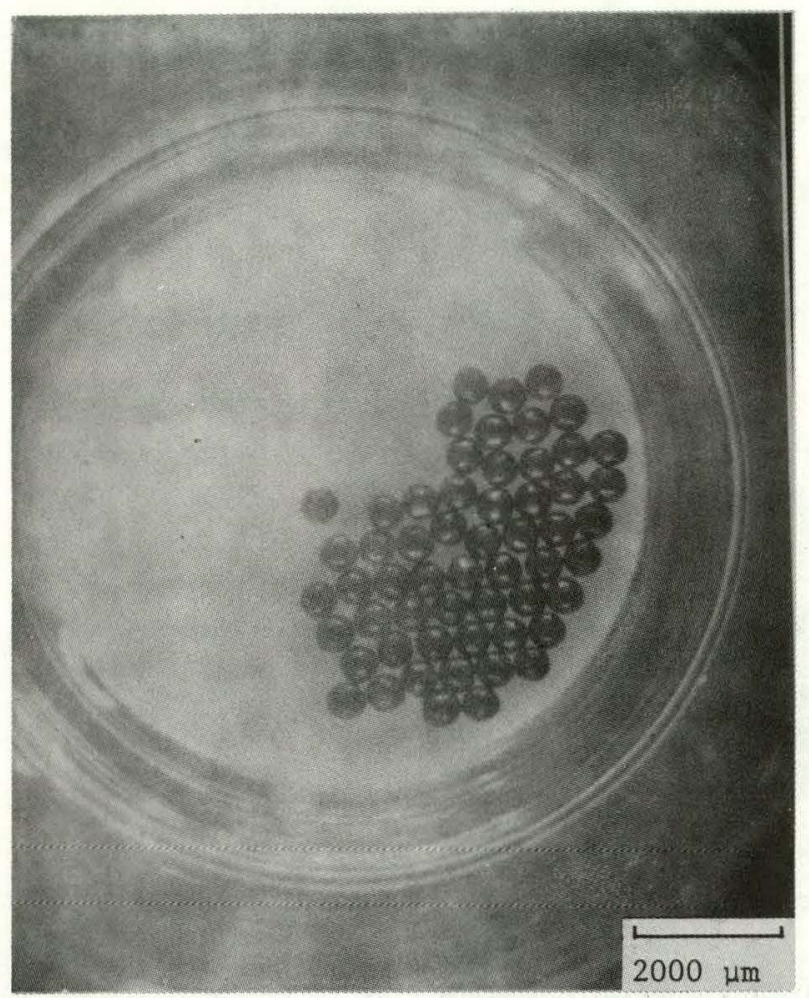

N78015-1

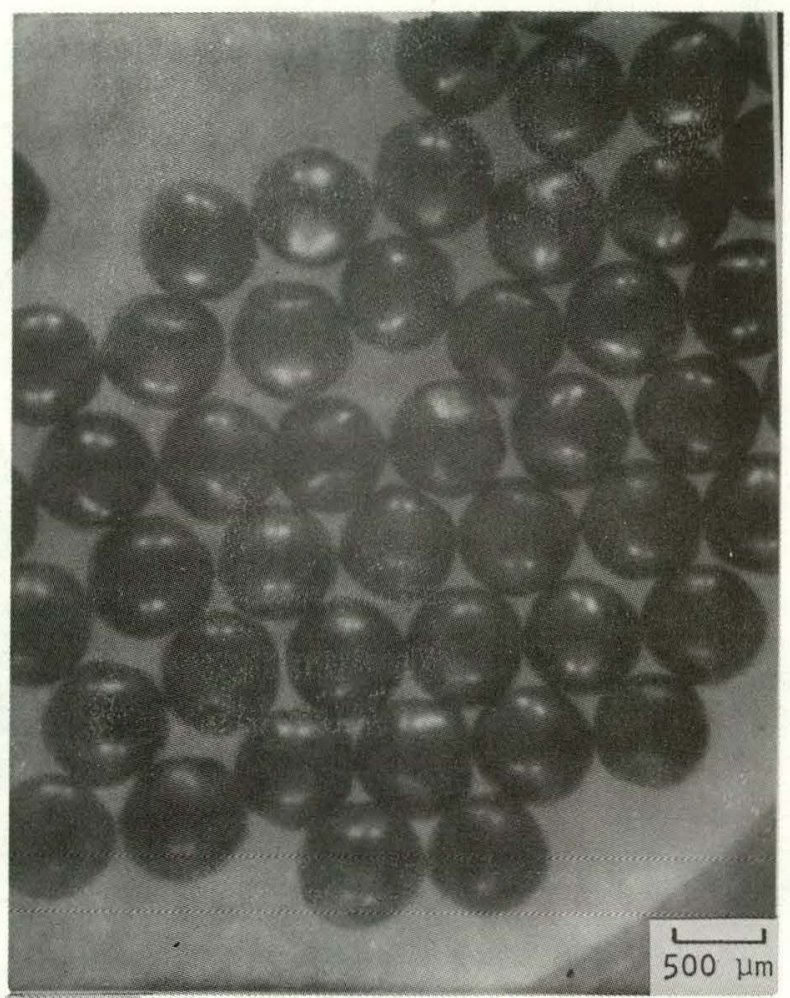

N78015-2

Fig. 5-2. Photomicrographs of a typical sample with zero pressure vessel and OPyC coating failure irradiated in the low temperature magazine; sample 8 had an average temperature of $1220^{\circ} \mathrm{C}$ and a fluence of $7.0 \times 10^{25} \mathrm{n} / \mathrm{m}^{2}(\mathrm{E}>29 \mathrm{fJ})$ HTGR. 


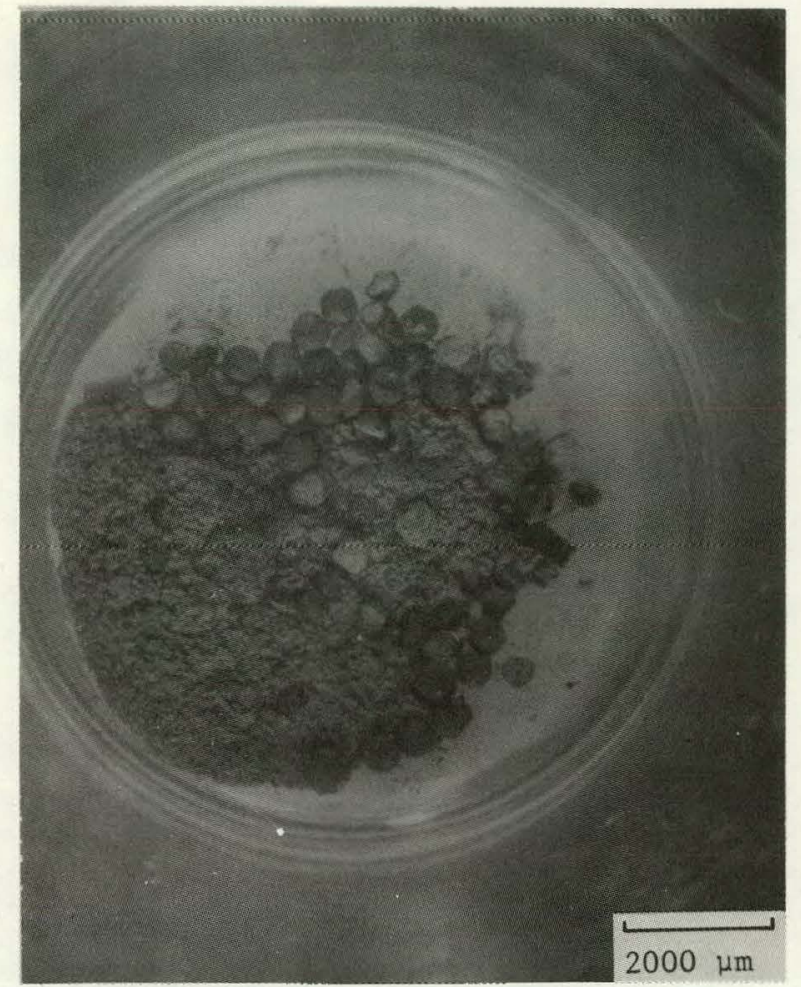

N78013-1

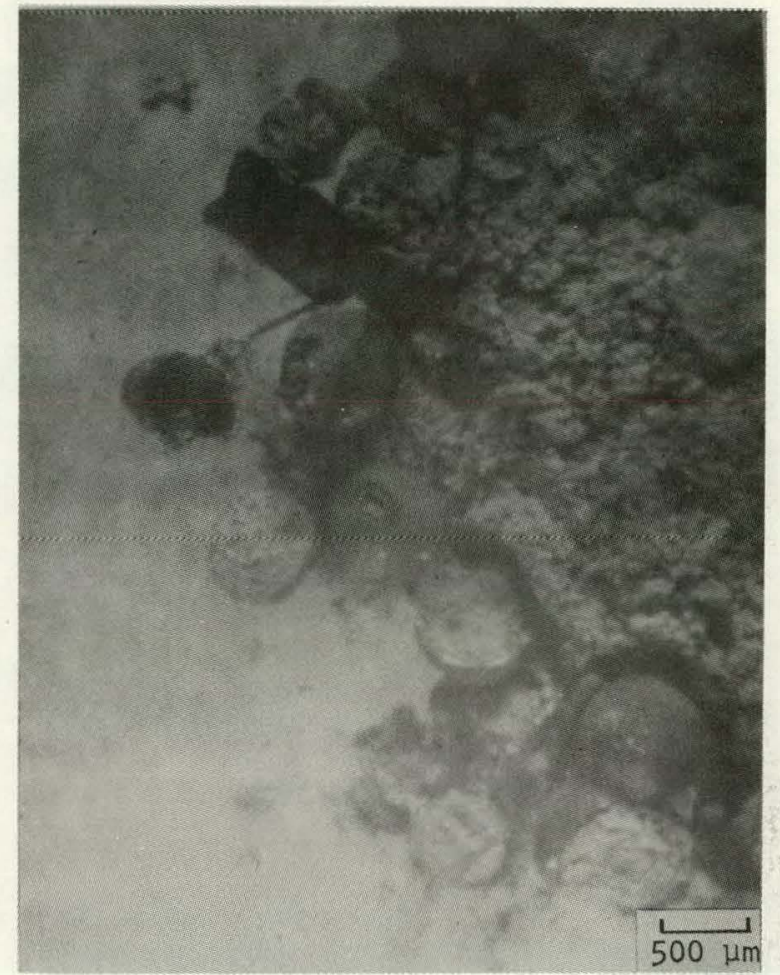

N78013-2

Fig. 5-3. Photomicrographs of total coating failure observed in a sample (6252-17-0161-001) irradiated at $1460^{\circ} \mathrm{C}$ to a fluence of $10.0 \times 10^{25} \mathrm{n} / \mathrm{m}^{2}(\mathrm{E}>29 \mathrm{fJ})_{\text {HTGR }}$ 


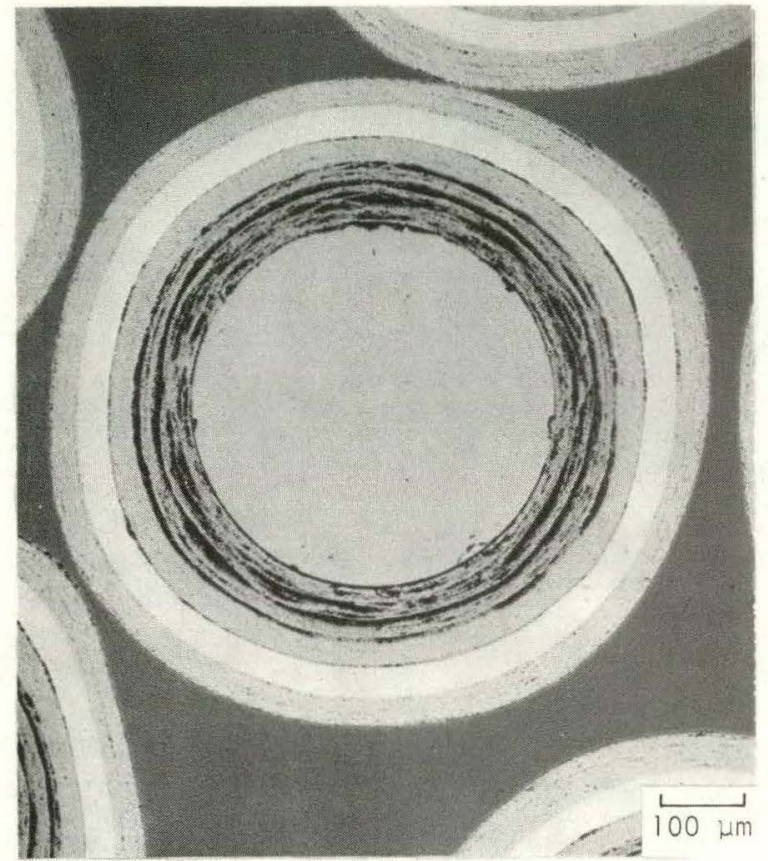

(a)

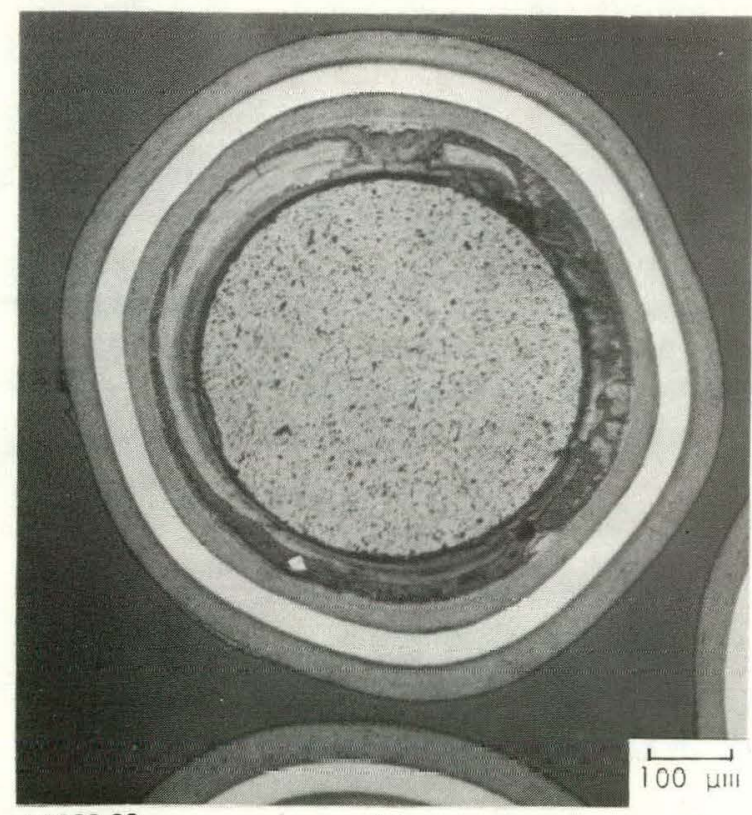

L7909-68 (c)

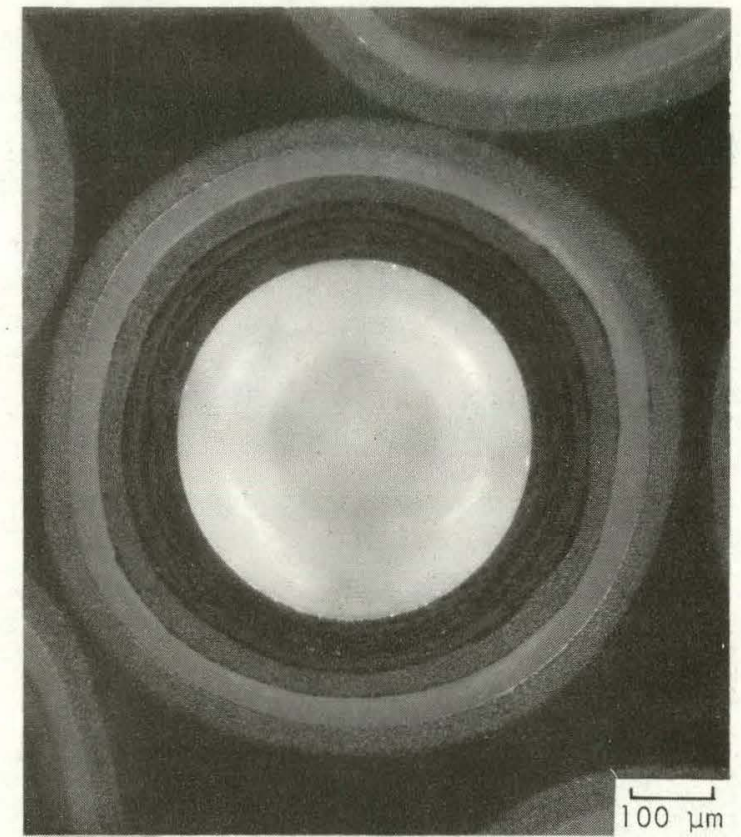

MP77008-8

(b)

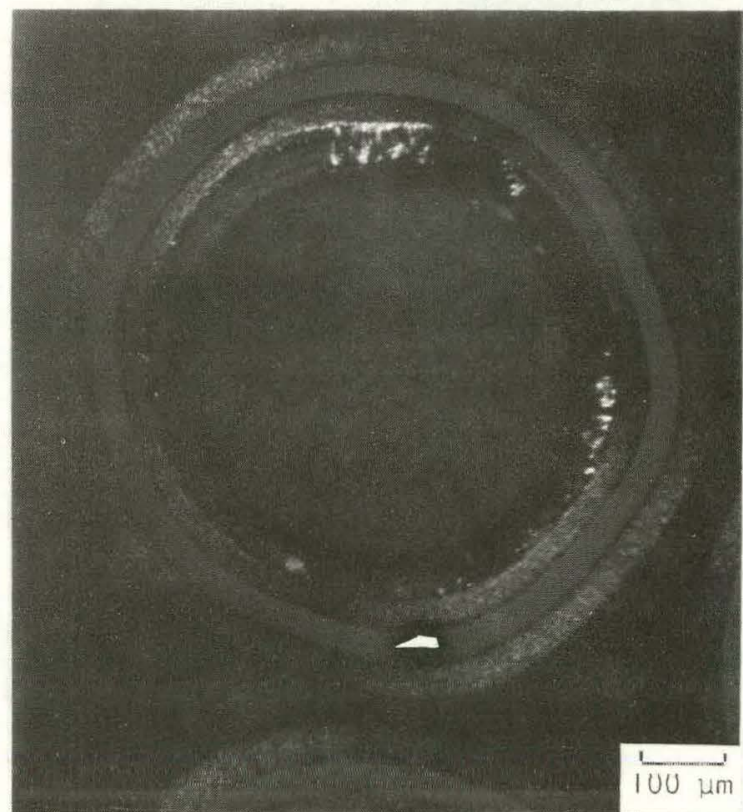

L7909-67

(d)

Fig. 5-4. Photomicrographs of sample 6252-14-0161-001: (a) and (b) as coated; (c) and (d) after irradiation at $1240^{\circ} \mathrm{C}$ to a fluence of $7.6 \times 10^{25} \mathrm{n} / \mathrm{m}^{2}$ (E > $29 \mathrm{fJ}$ ) HTGR; (a) and (c) are bright field; (b) and (d) are polarized light. Note redeposition of the buffer in (c). 


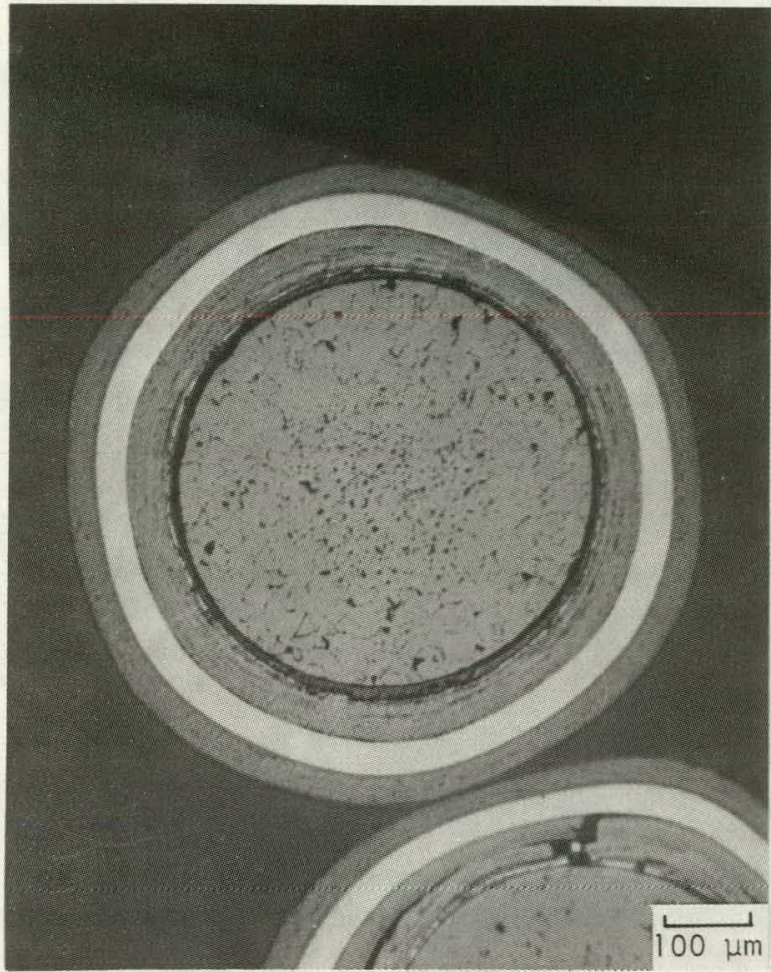

L7907-47

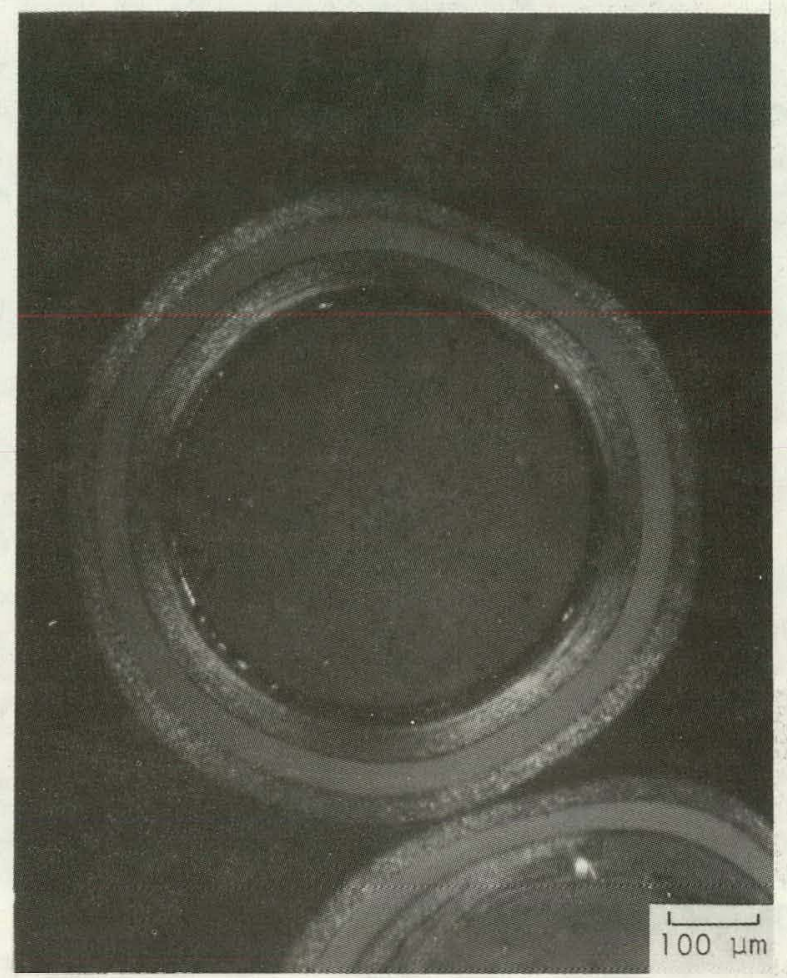

L7909-48

Fig. 5-5. Photomicrographs of typical particle with a 27- $\mu$ m-thick buffer (6252-14-0261-002) irradiated at $1210^{\circ} \mathrm{C}$ to a burn-up of $6.0 \%$ FIMA 


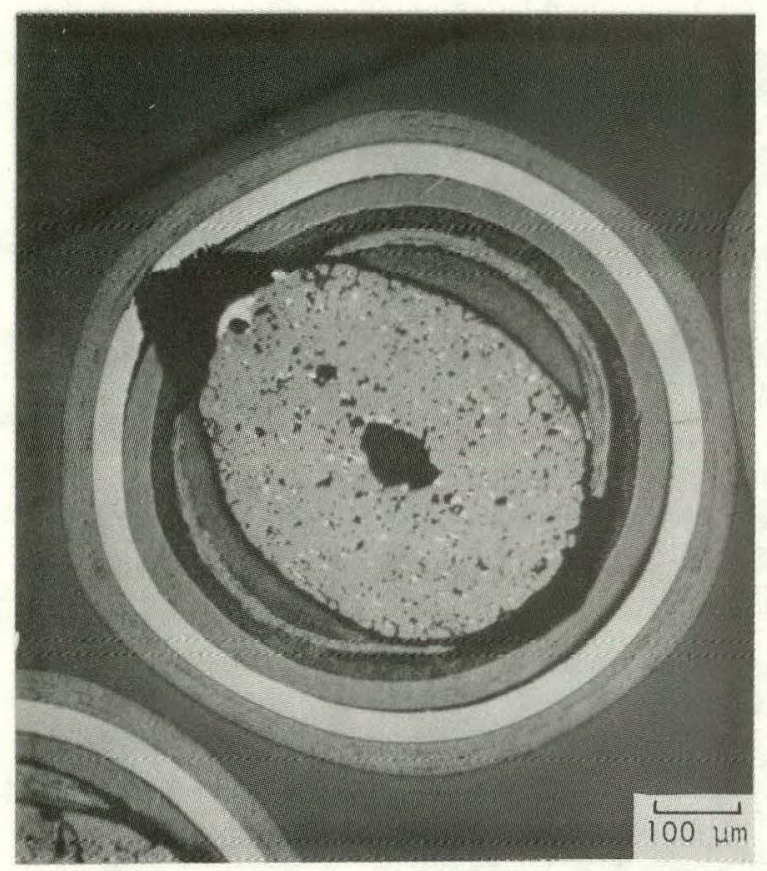

L7909-32

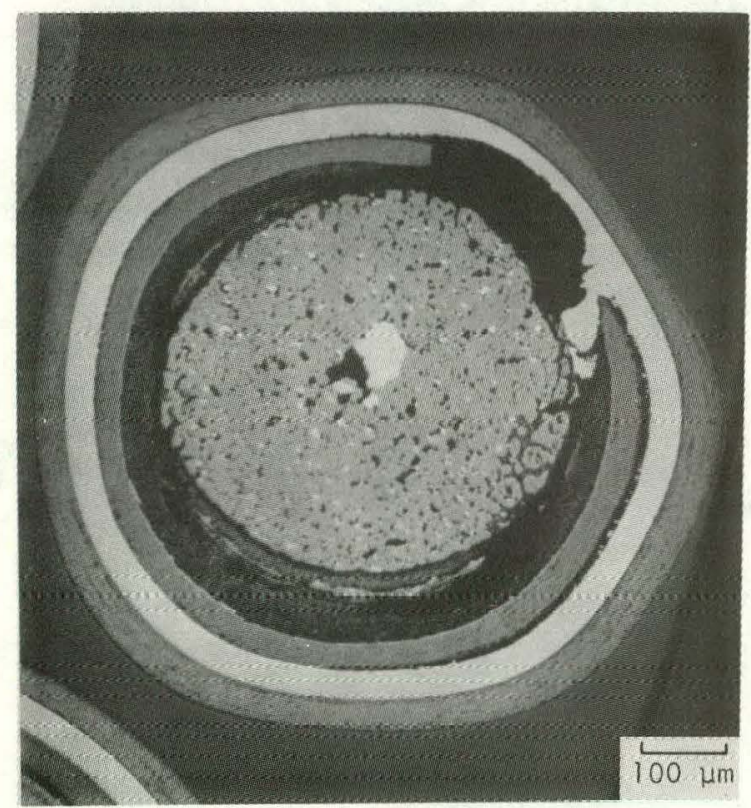

L7909-35

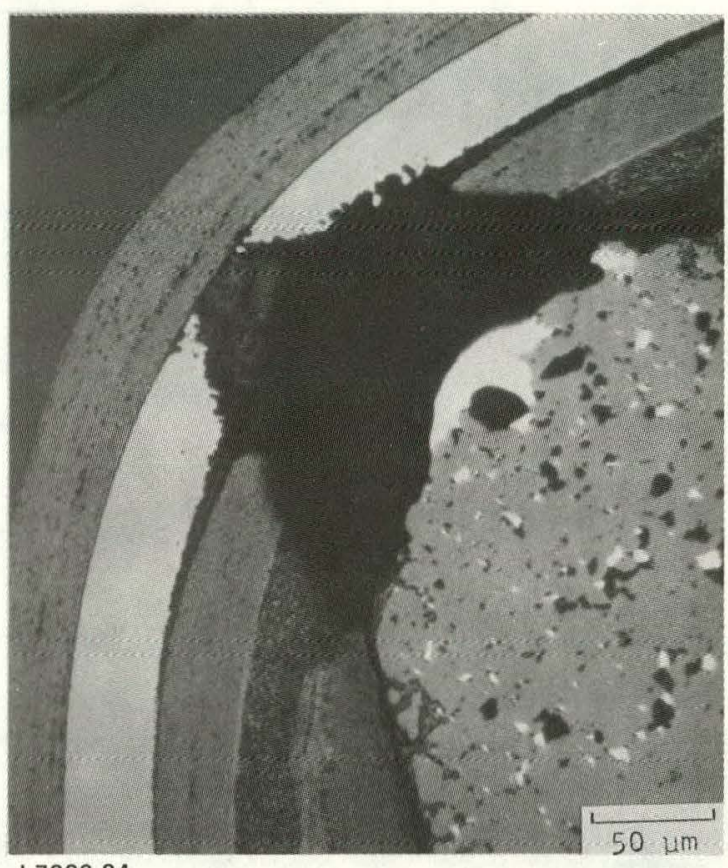

L7909-34

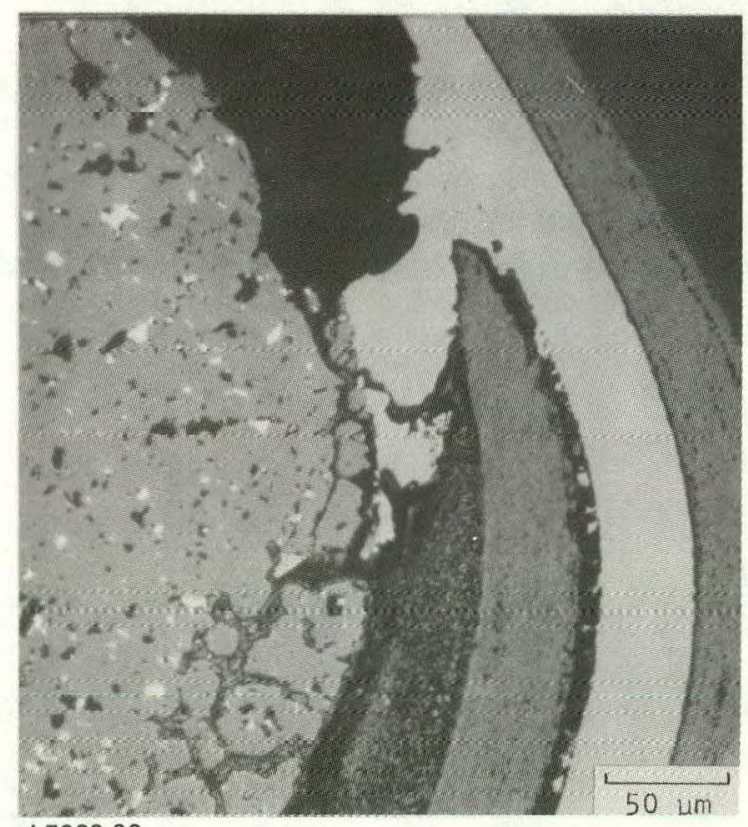

L7909-36

Fig. 5-6. Photomicrographs of two particles (6252-07-0262-002) irradiated at $1430^{\circ} \mathrm{C}$ to a burnup of $10.9 \%$ FIMA and a fluence of $9.4 \mathrm{x}$ $10^{25} \mathrm{n} / \mathrm{m}^{2}$ (E > $\left.29 \mathrm{fJ}\right)_{\text {HTGR }}$ 

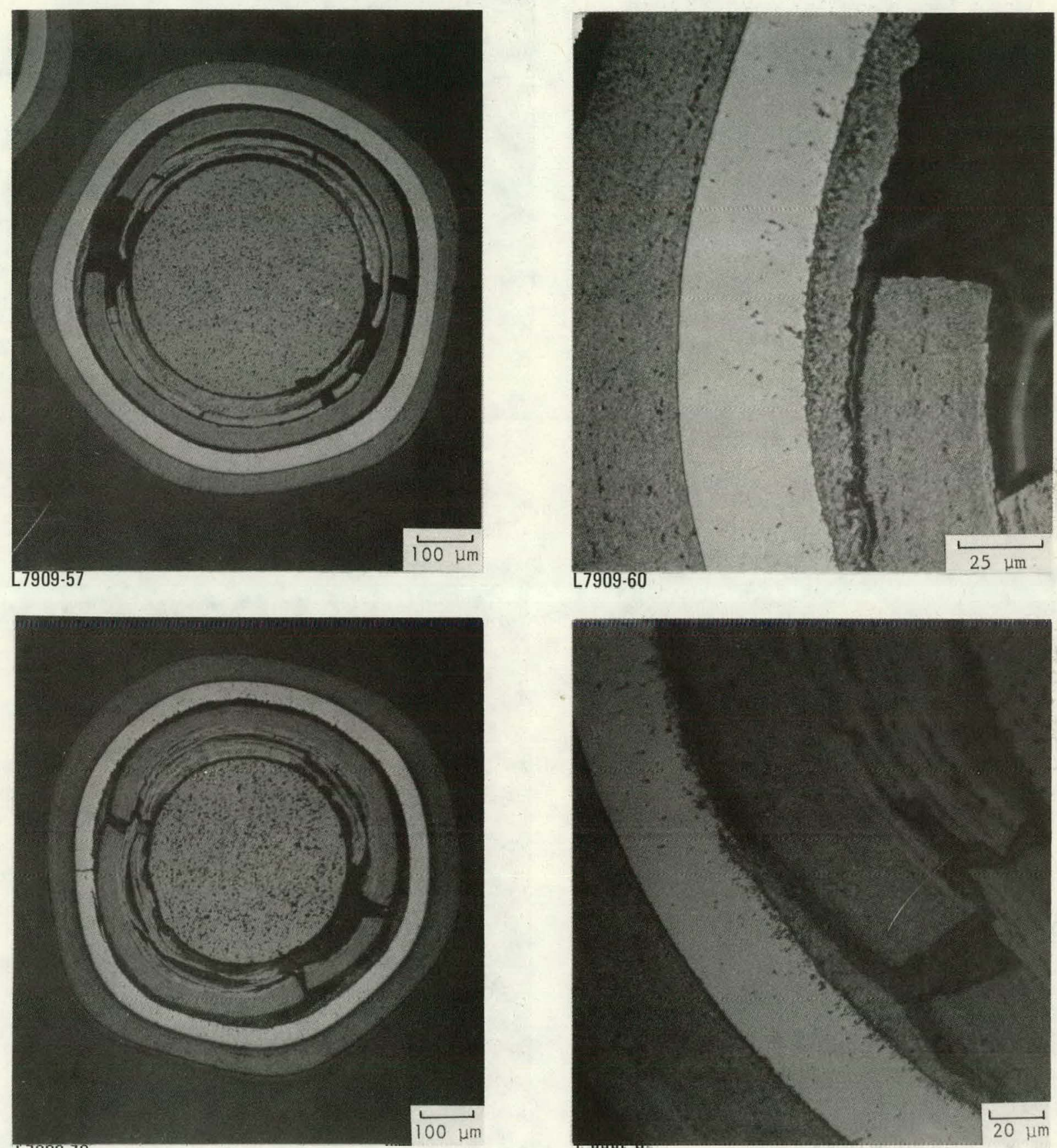

L7909-76

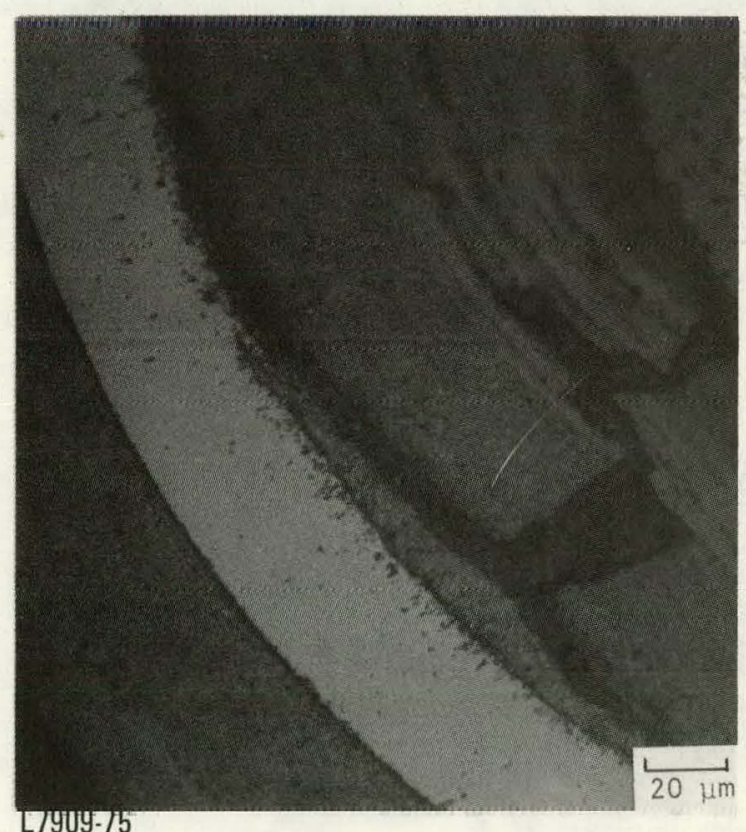

Fig. 5-7. Photomicrographs of representative particles irradiated at $1240^{\circ} \mathrm{C}$ to a burn-up of $7.9 \%$ FIMA which showed SiC coatings attacked around the circumference of the coating 


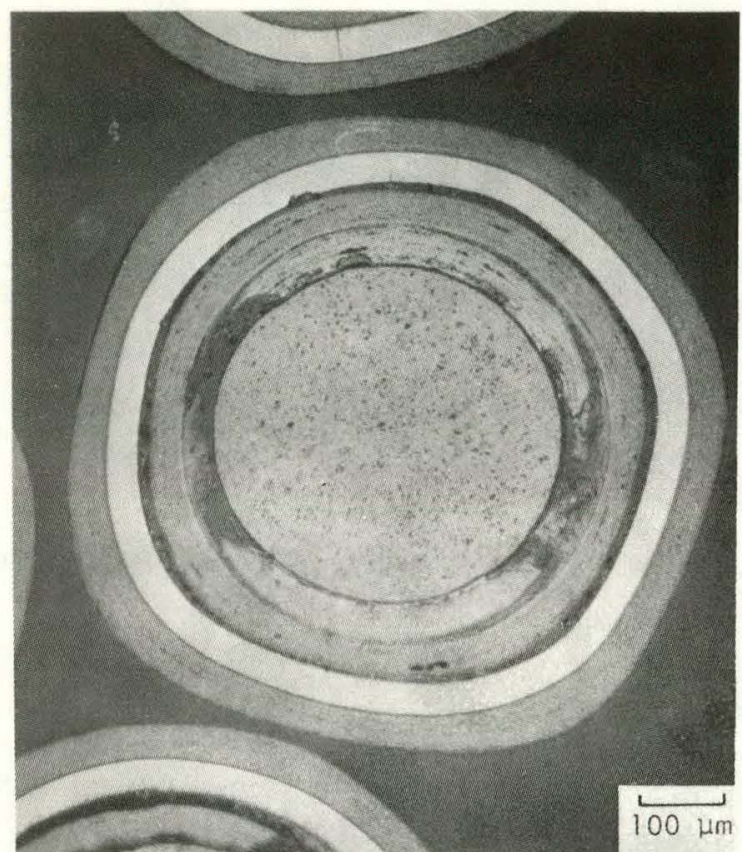

L7090-14

(a)

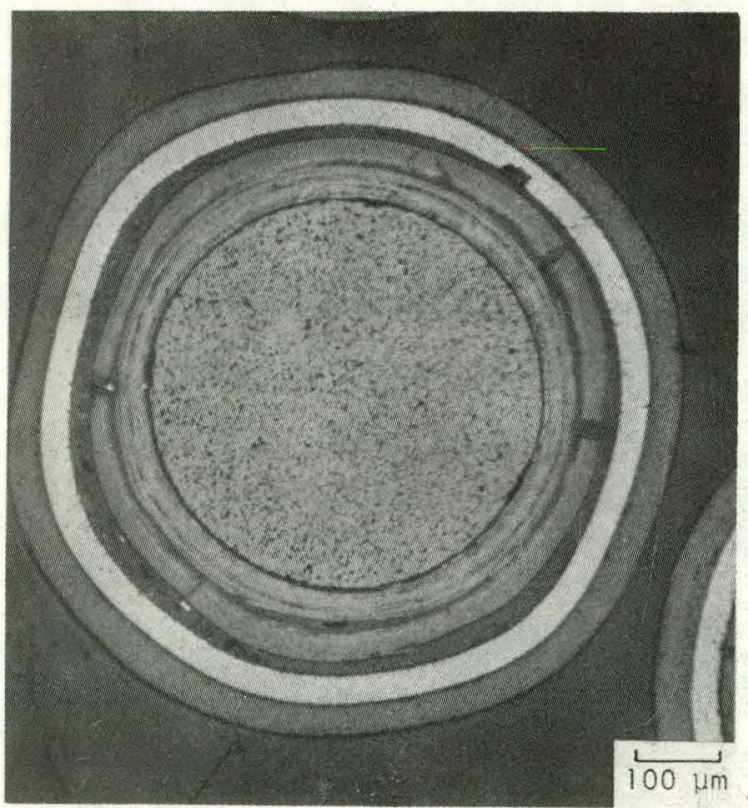

L7909-71

(c)

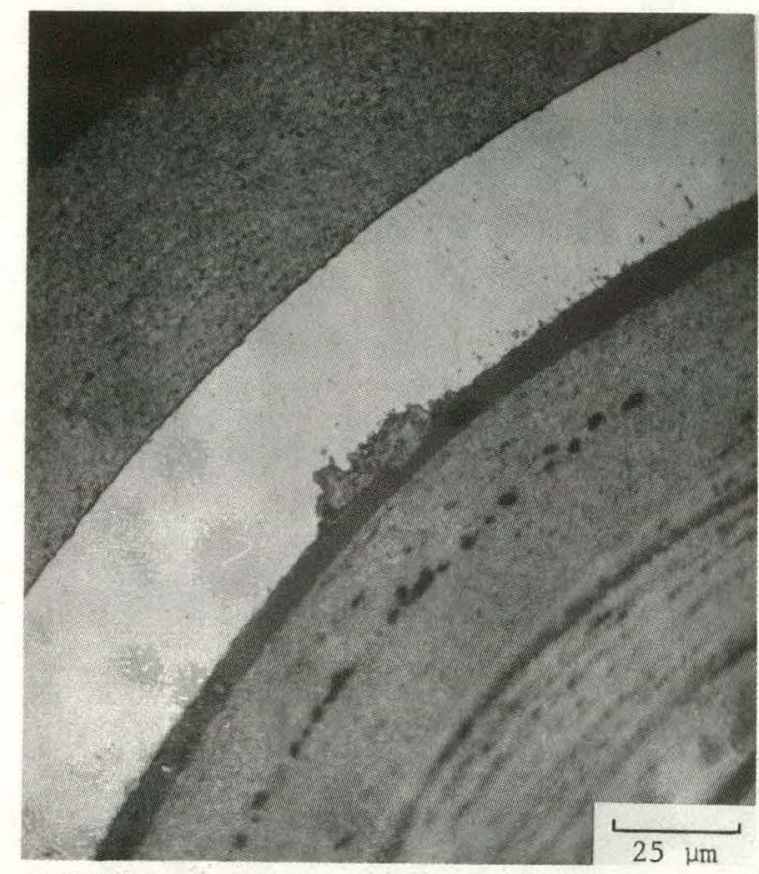

(b)

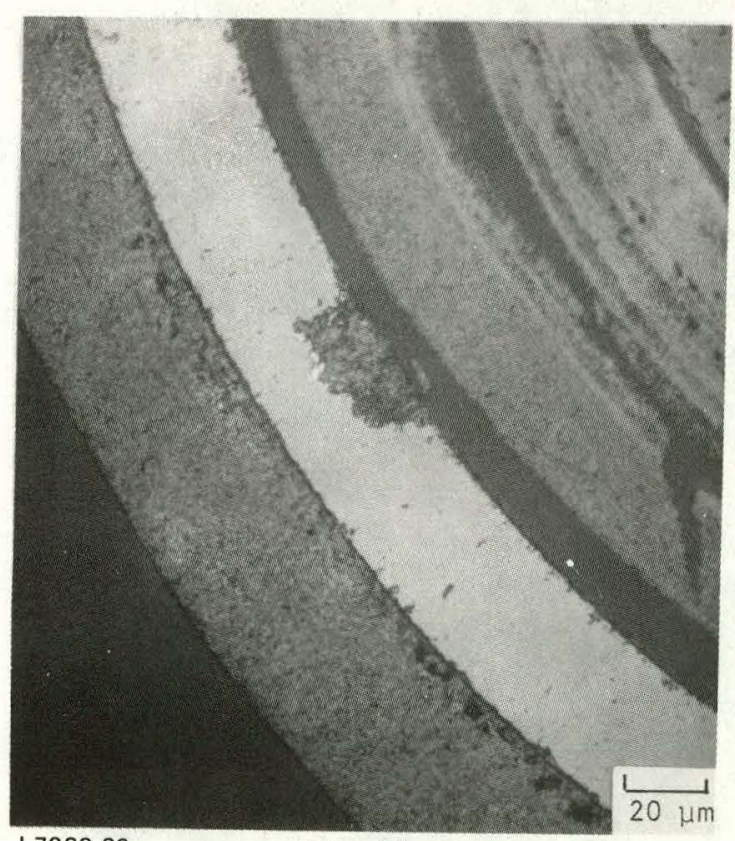

L7909-69

Fig. 5-8. Photomicrographs of two particles irradiated at $1240^{\circ} \mathrm{C}$ to a burn-up of $7.9 \%$ FIMA showing a localized attack of the SiC coating 


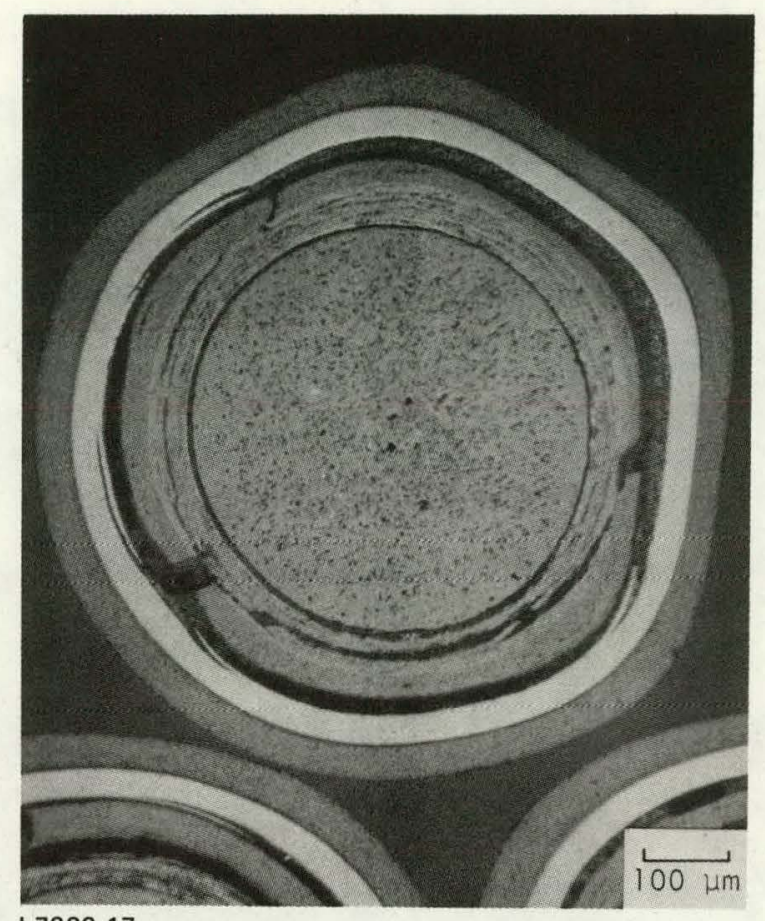

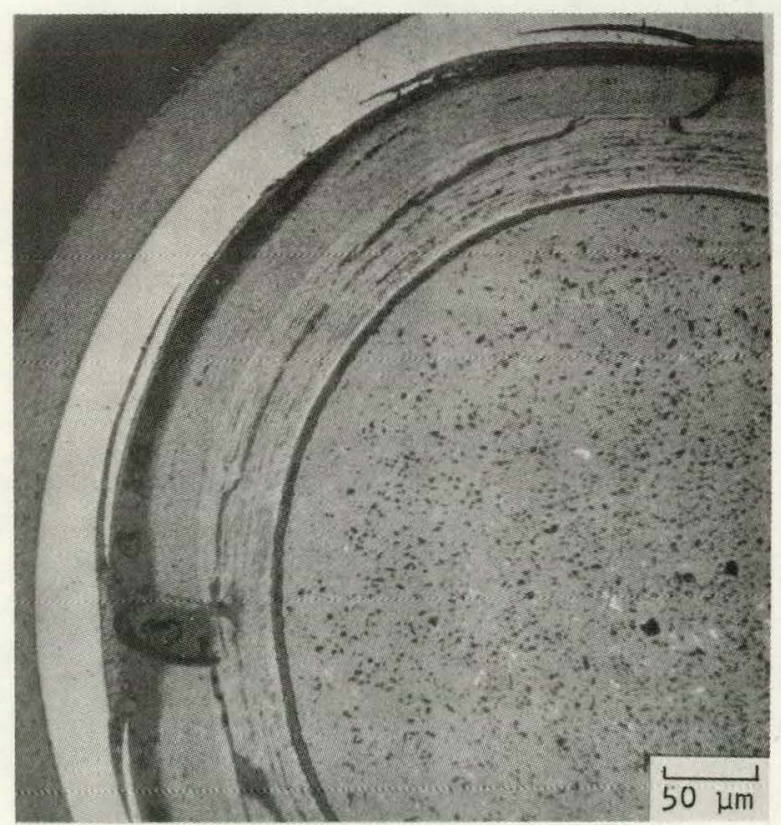

L7909-18

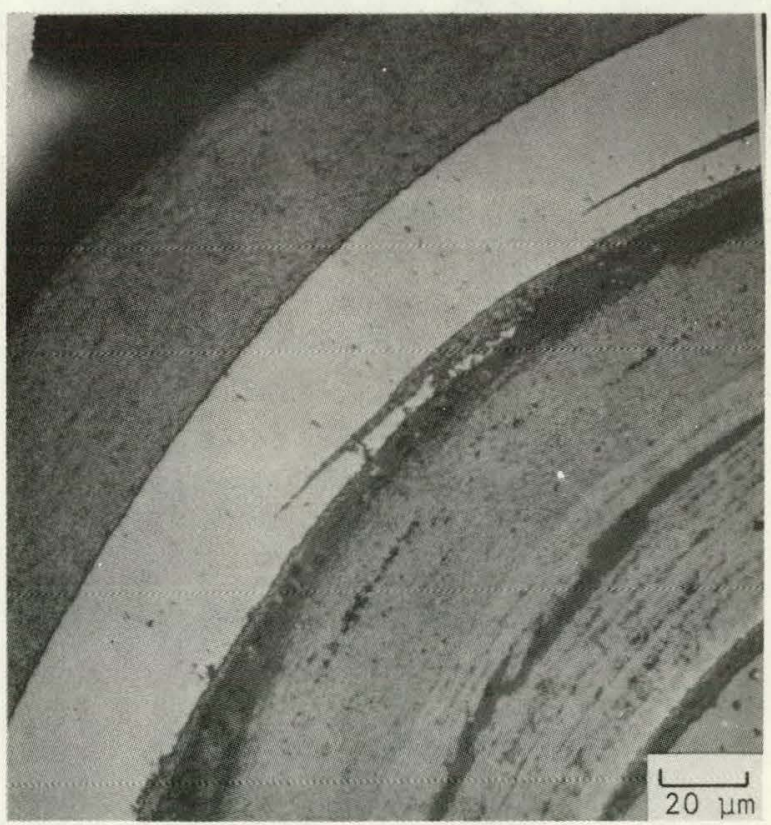

L7909-19

Fig. 5-9. Photomicrograph of fracturing of the SiC coating near the inner surface of particles irradiated at $1180^{\circ} \mathrm{C}$ to a fluence of $8.2 \times 10^{25} \mathrm{n} / \mathrm{m}^{2}(\mathrm{~F}>29$ f.I) HTGR 


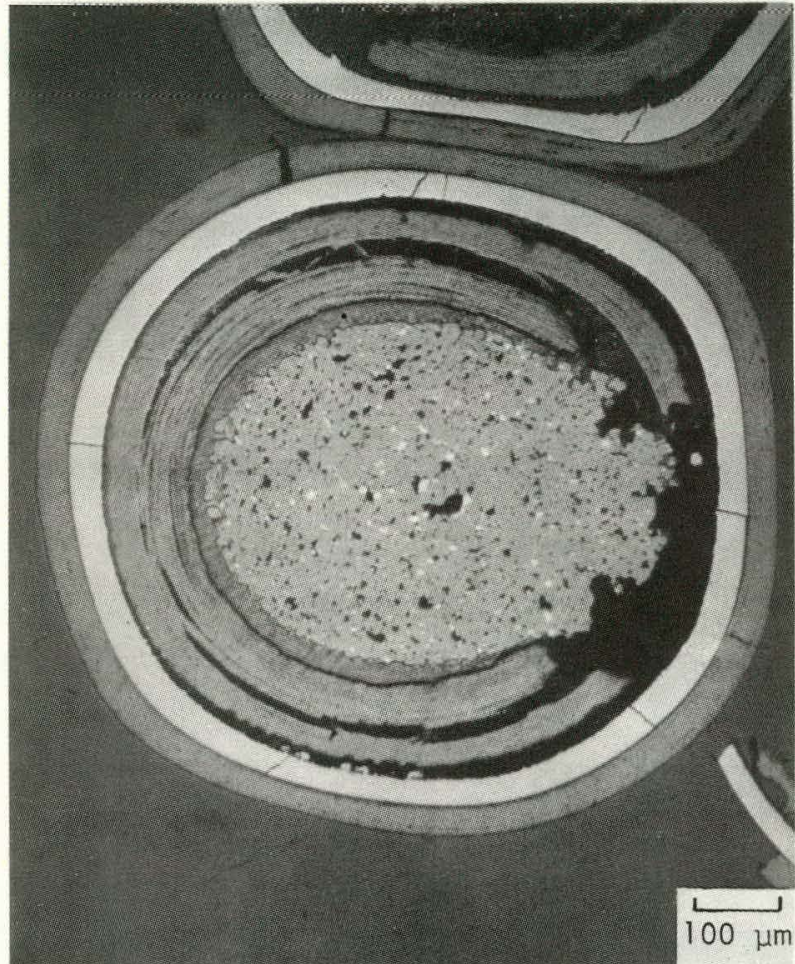

L7909-1

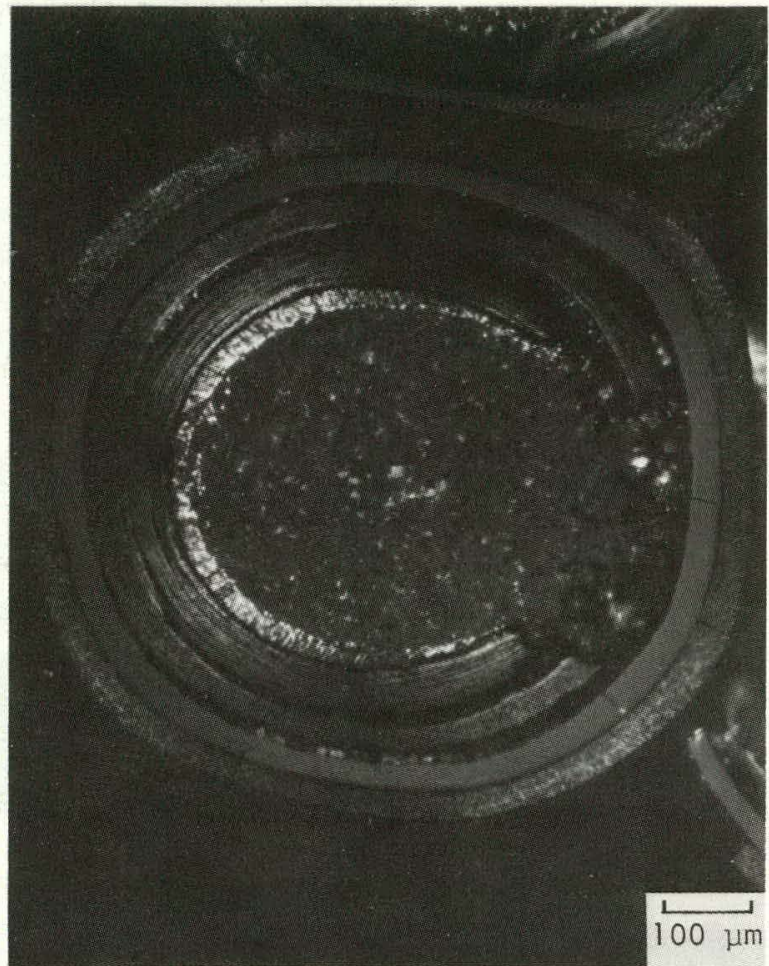

L7909-2 (b)

Fig. 5-10. Photomicrographs of kernel migration of a particle irradiated at $1490^{\circ} \mathrm{C}$ to a burn-up of $10.5 \%$ FIMA: (d) bright field;

(b) polarized light 

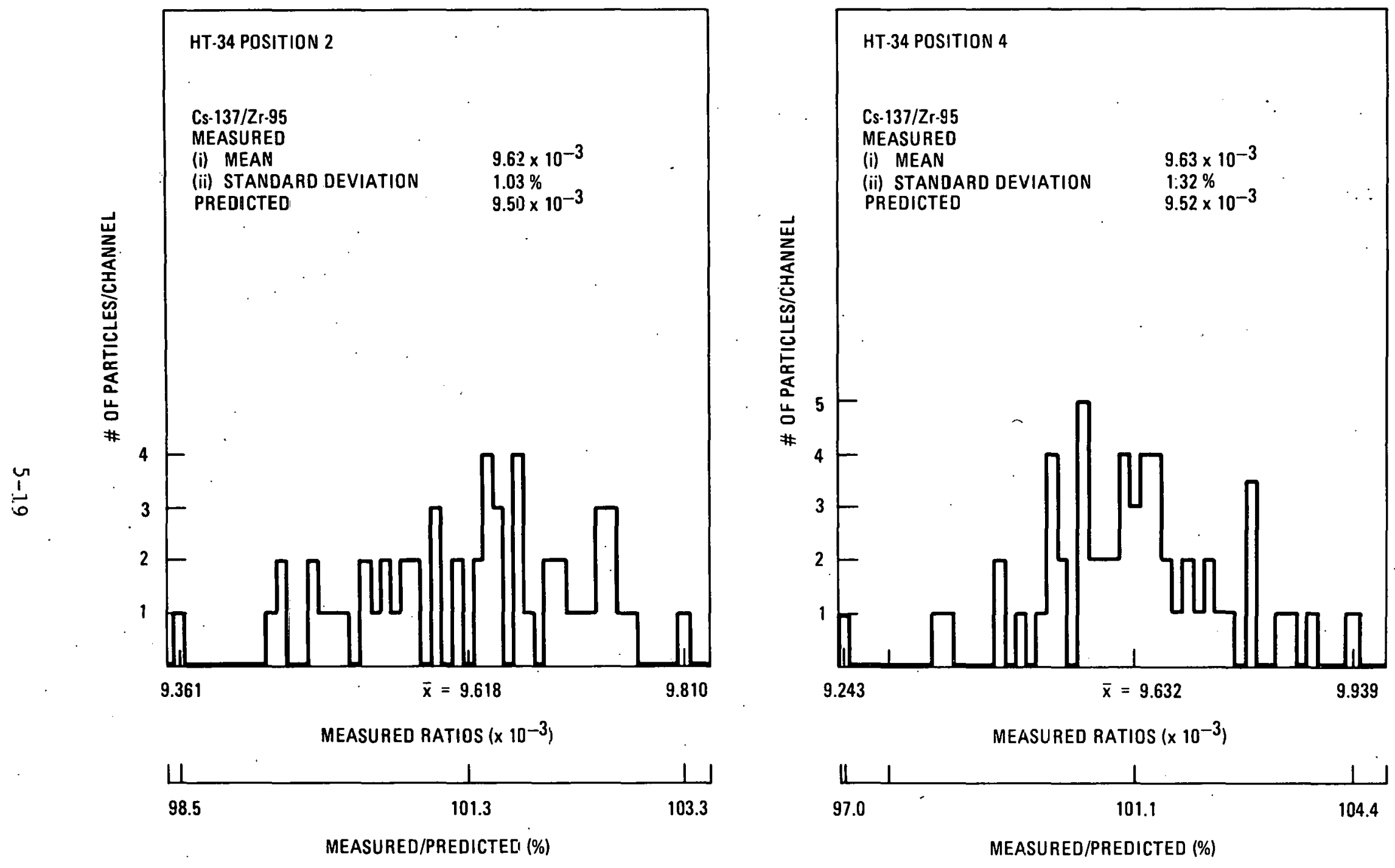

Fig. 5-1la. Histograms of the measured $\mathrm{Cs}-137 / \mathrm{Zr}-95 \mathrm{ratios}$. The measured/predicted Cs/Zr ratios are also given (NOTE: the scale is different for each sample). 

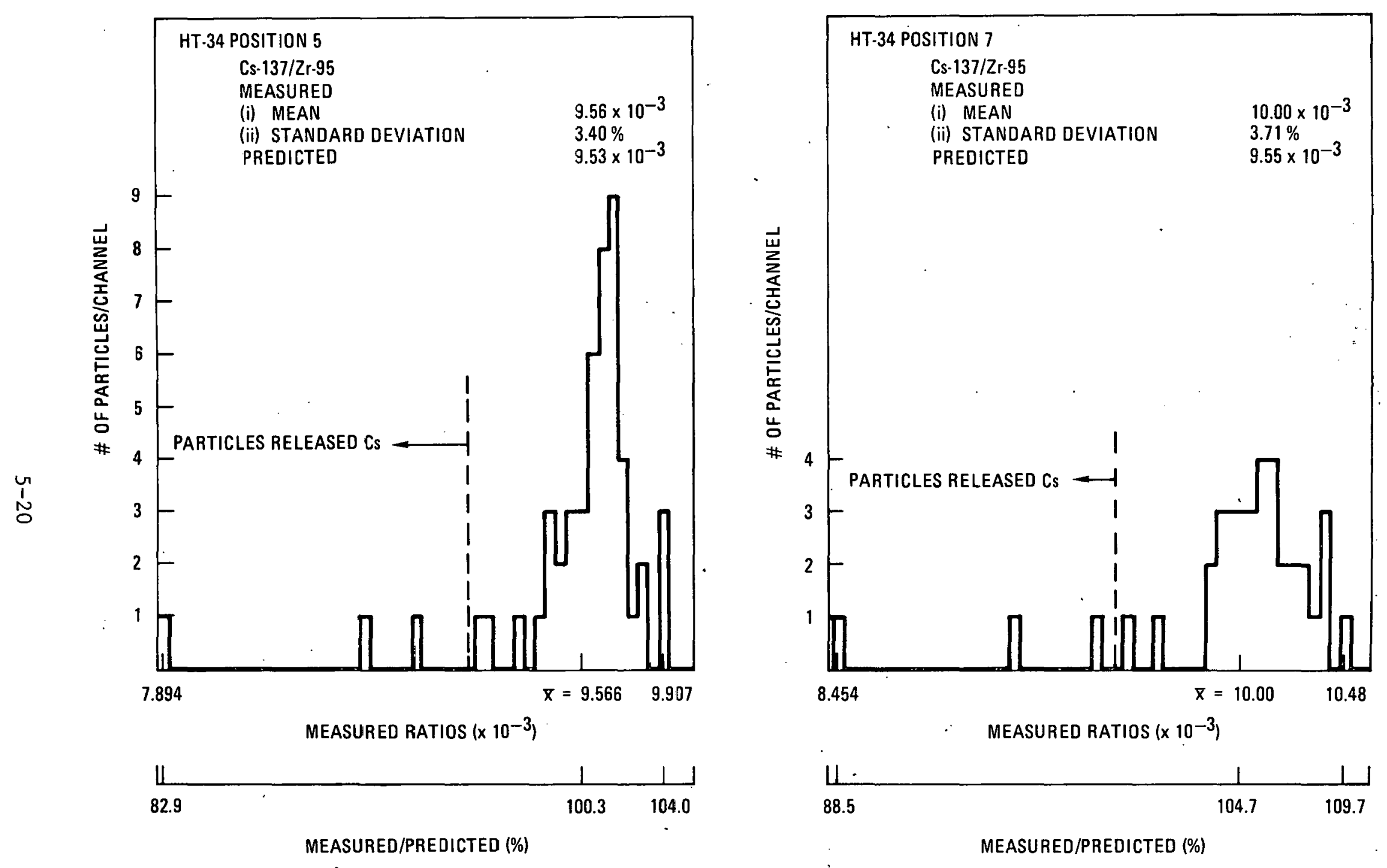

Fig. 5-11b. Histograms of Cs $-137 / \mathrm{Zr}-95$ ratios. The measured/predicted Cs/Zr ratios are also given. The figure indicates the particles that released Cs. (NOTE: the scale is different for each sample). 

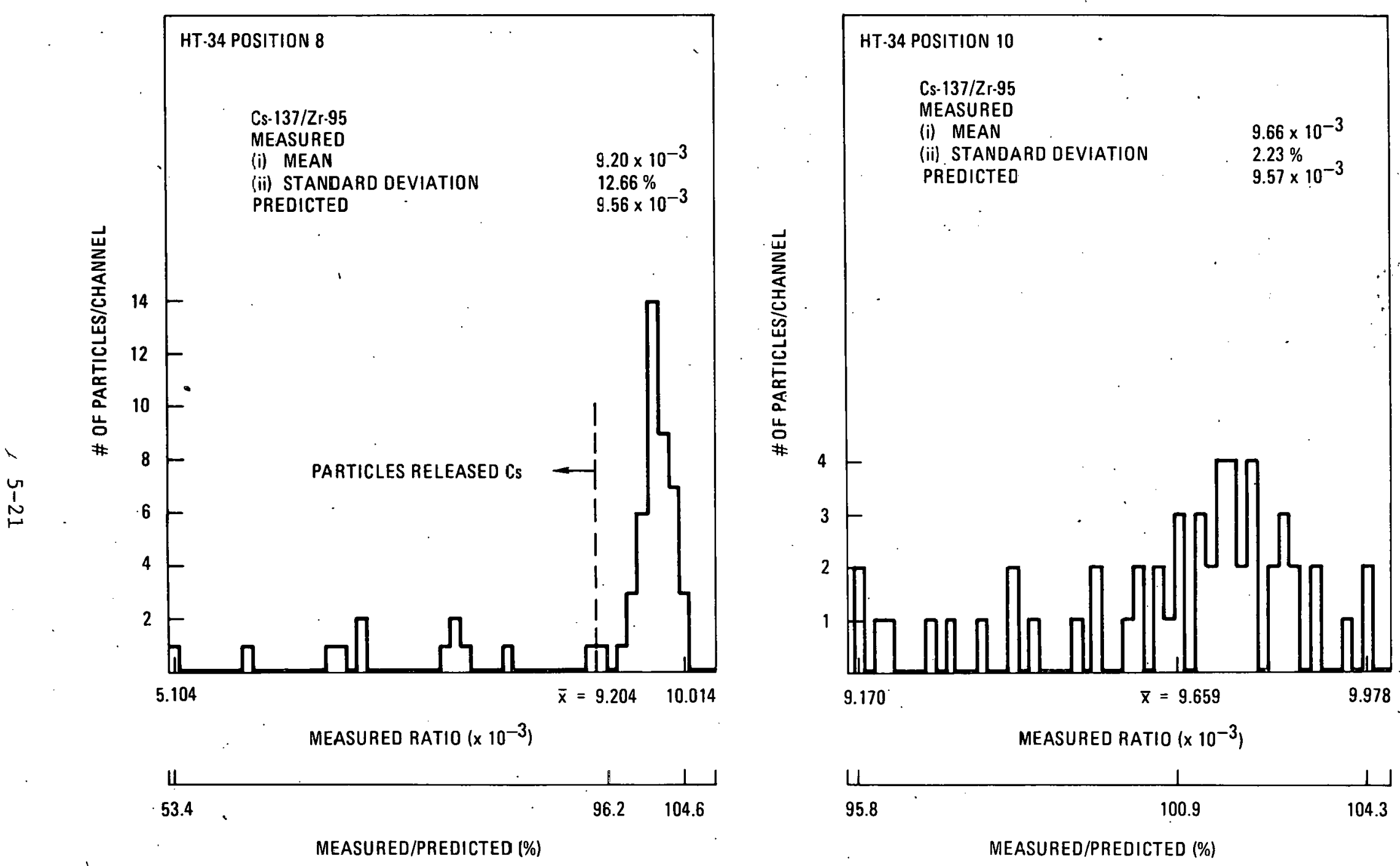

Fig. 5-11c. Histograms of Cs-137/Zr-95 ratios. The measured/predicted Cs/2r ratios are also given. The figure indicates the particles that released Cs. (NOTE: the scale is different for each sample). 

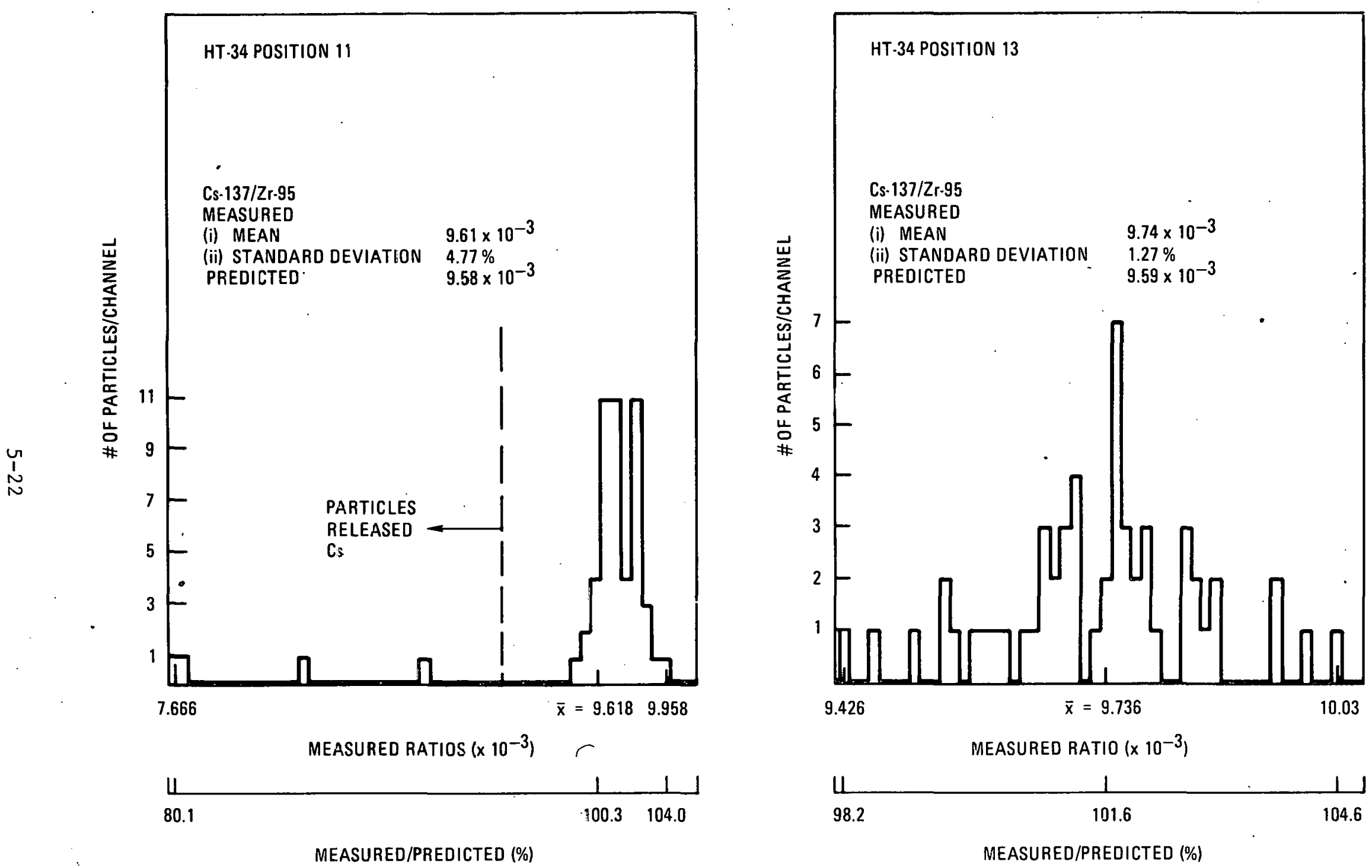

Fig. 5-Ild. Histograms of $\mathrm{Cs}-137 / \mathrm{Zr}-95$ ratios. The measured/predicted Cs/Zr ratios are also given. The figure indicates the particles that released Cs. (NOTE: the scale is different for each sample). 

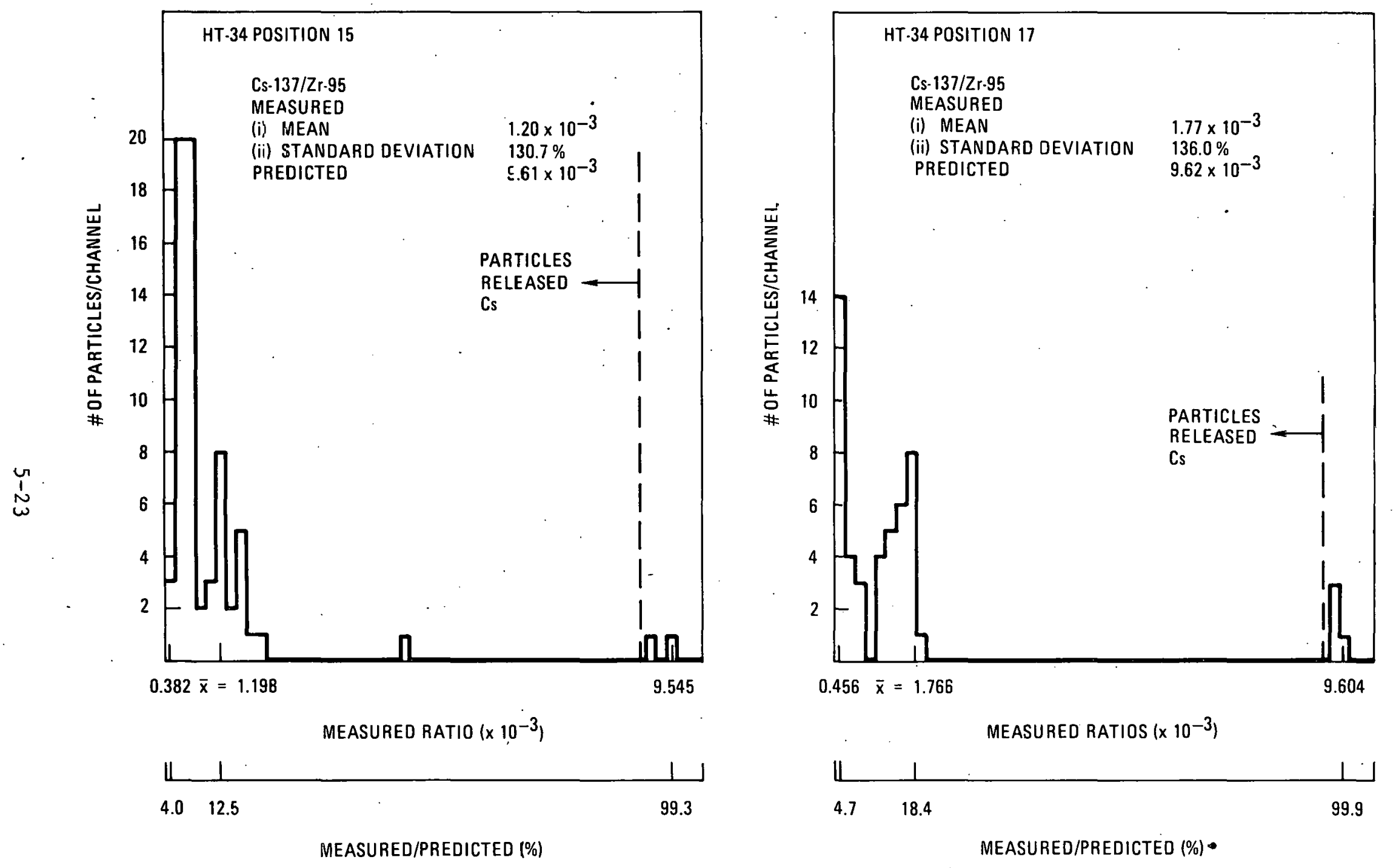

Fig. 5-1le. Histograms of Cs-137/2r-95 ratios. The measured/predicted Cs/Zr ratios are also given. The figure indicates the particles that released Cs. (NOTE: the scale is different for each sample). 

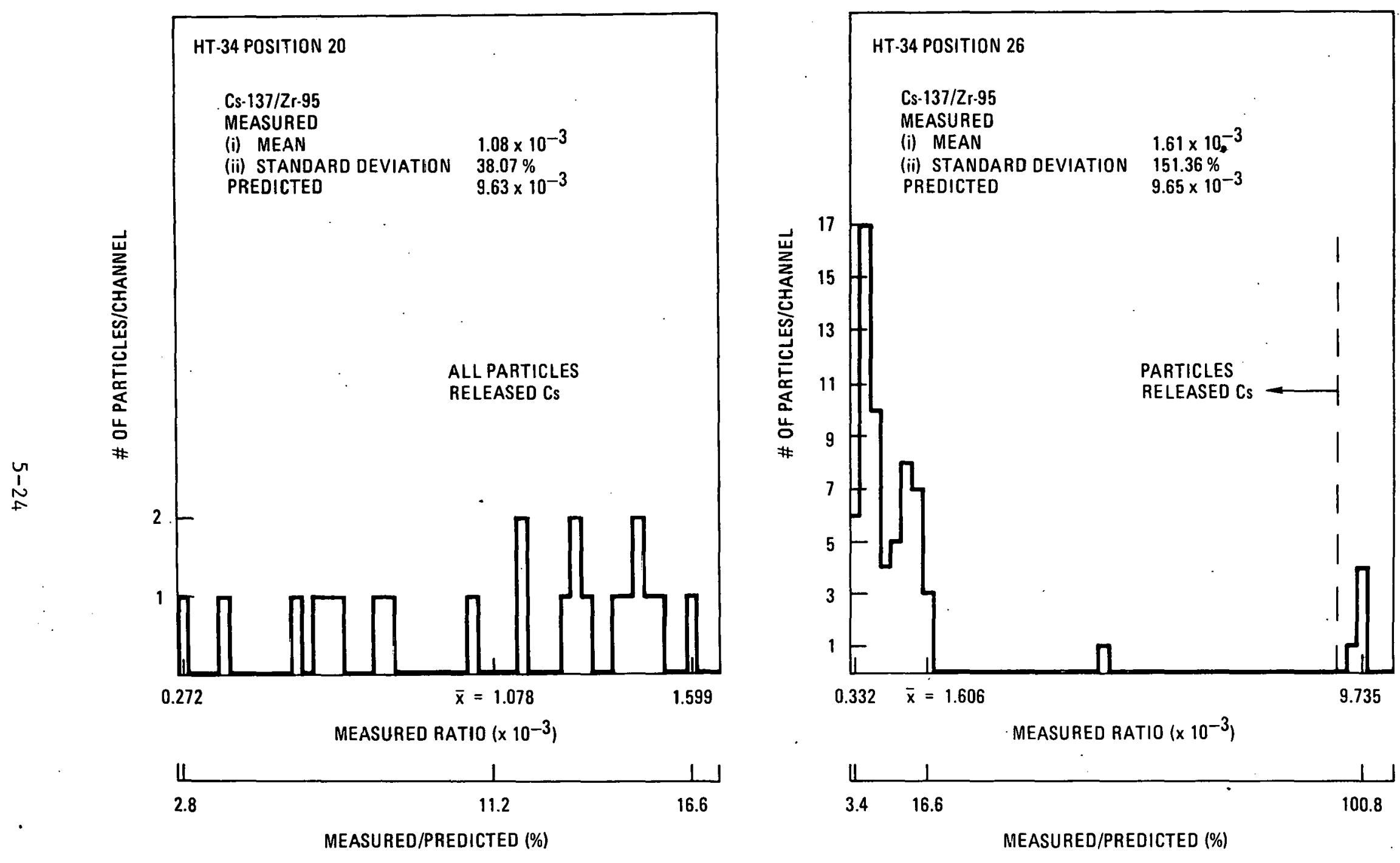

Fig. 5-11f. Histograms of Cs-137/2r-95 ratios. The measured/predicted Cs/Zr ratios are also given. The figure indicates the particles that released Cs. (NOTE: the scale is different for each sample). 

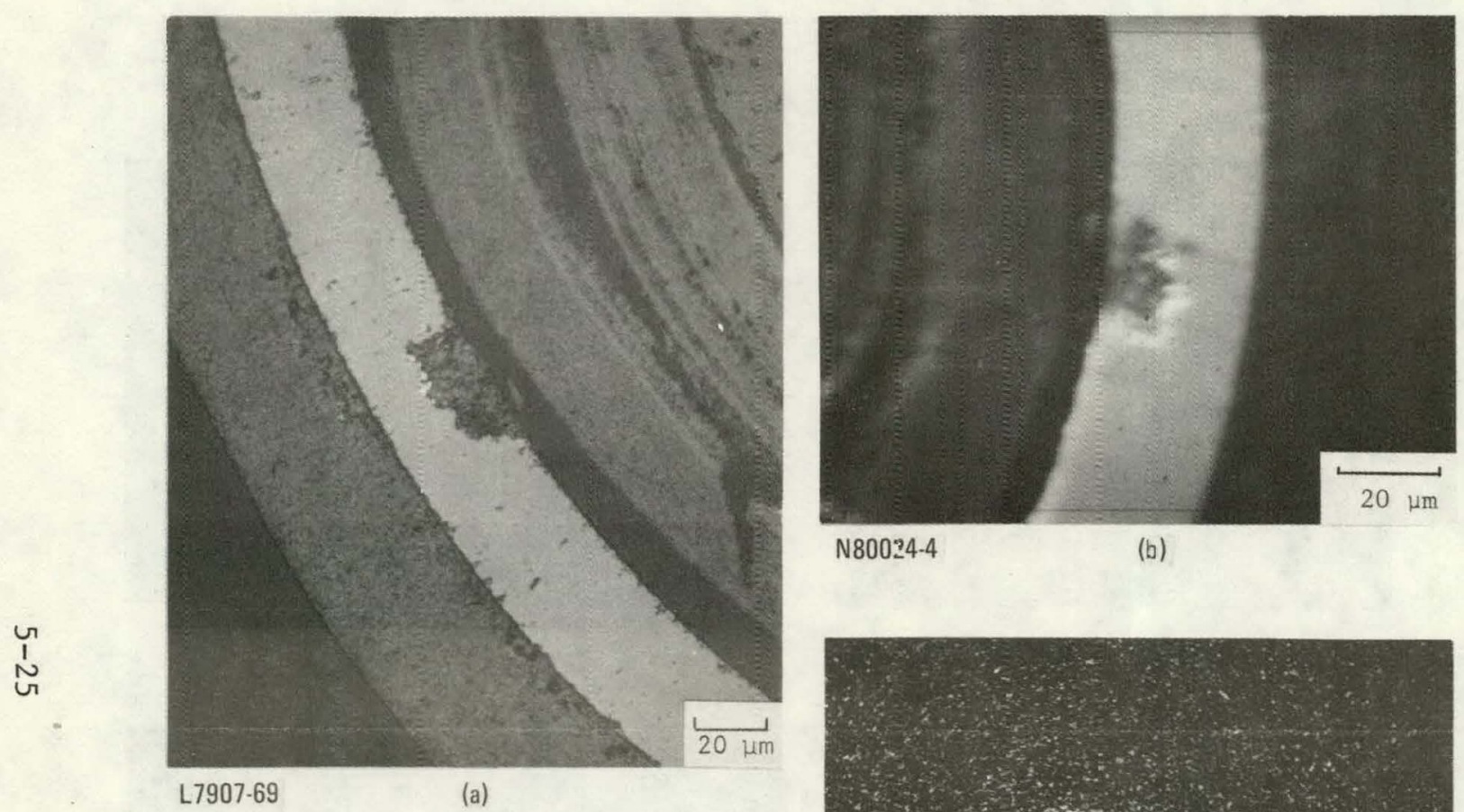

(b)
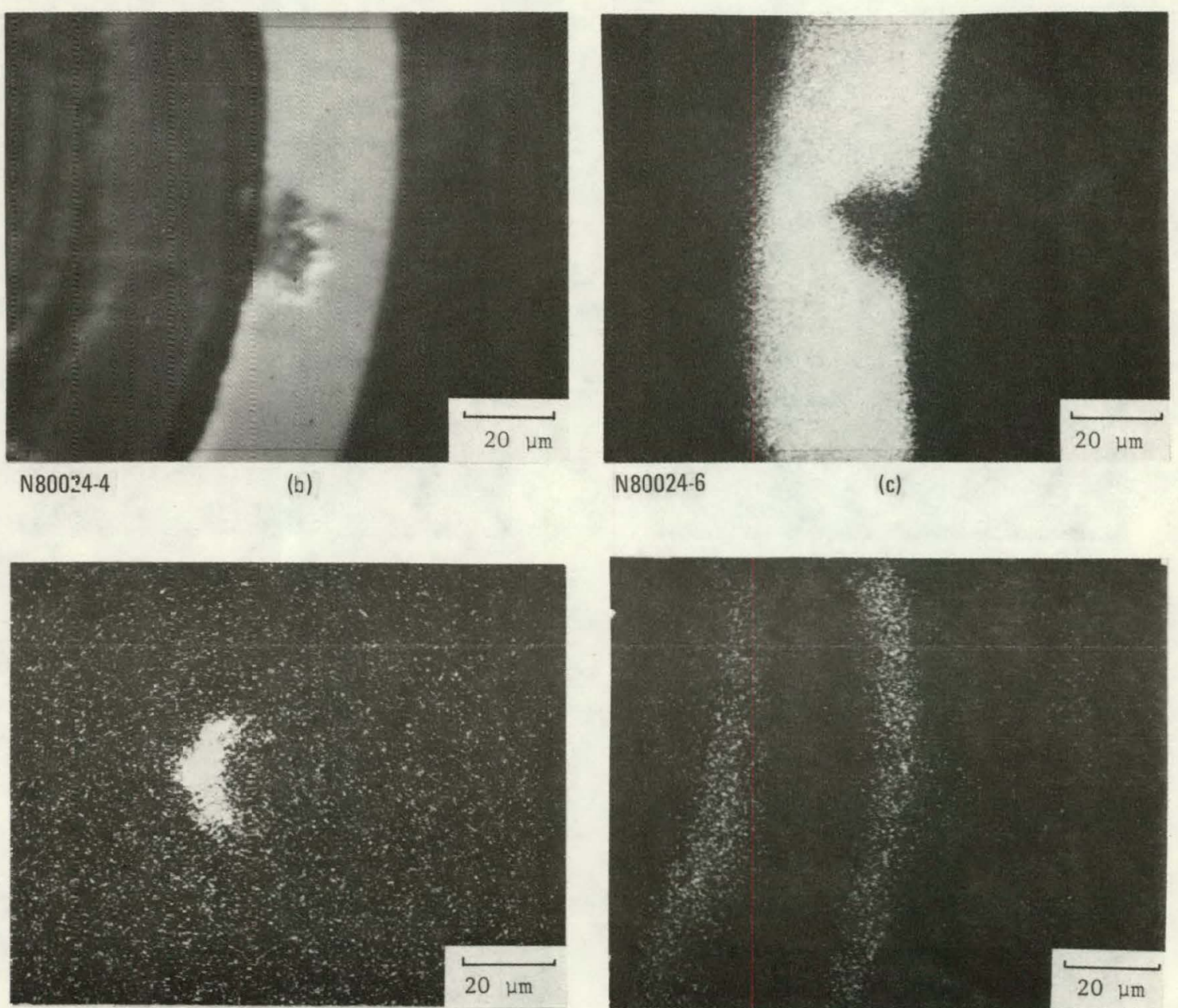

N80024-3

(d)

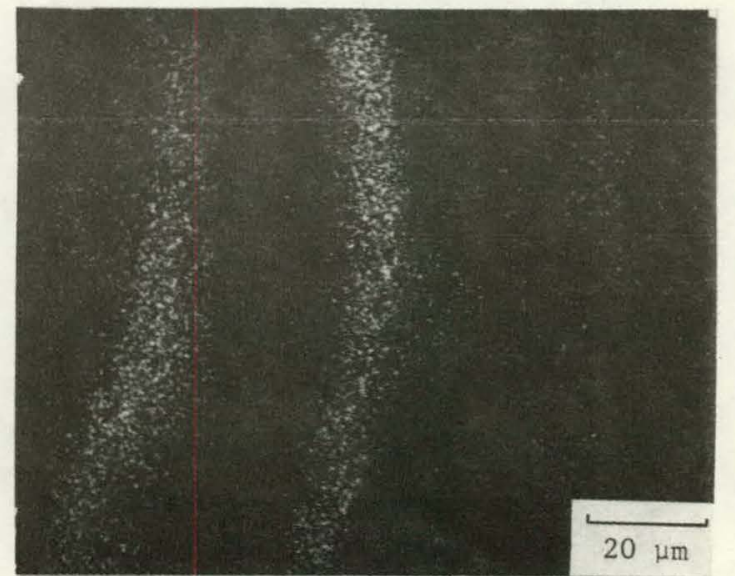

N80024-5

(e)

Fig. 5-12. Results of electron microprobe analysis o= a SiC reaction zone of a particle (6252-14-0161-001) irradiated at $1240^{\circ} \mathrm{C}$ to a turnup of $7.6 \%$ IIMA and a fluence of $7.5 \mathrm{x} 10^{25} \mathrm{n} / \mathrm{m}^{2}$ (E $>29 \mathrm{fJ}$ ) $\mathrm{HTGR}$ (a) Cptical photomicrograpl; (b) back-scattered electron image; (c) $\mathrm{Si} \mathrm{K \alpha ;} \mathrm{(d)} \mathrm{Pd} \mathrm{L \alpha ;} \mathrm{(e)} \mathrm{C1} \mathrm{K \alpha}$ 

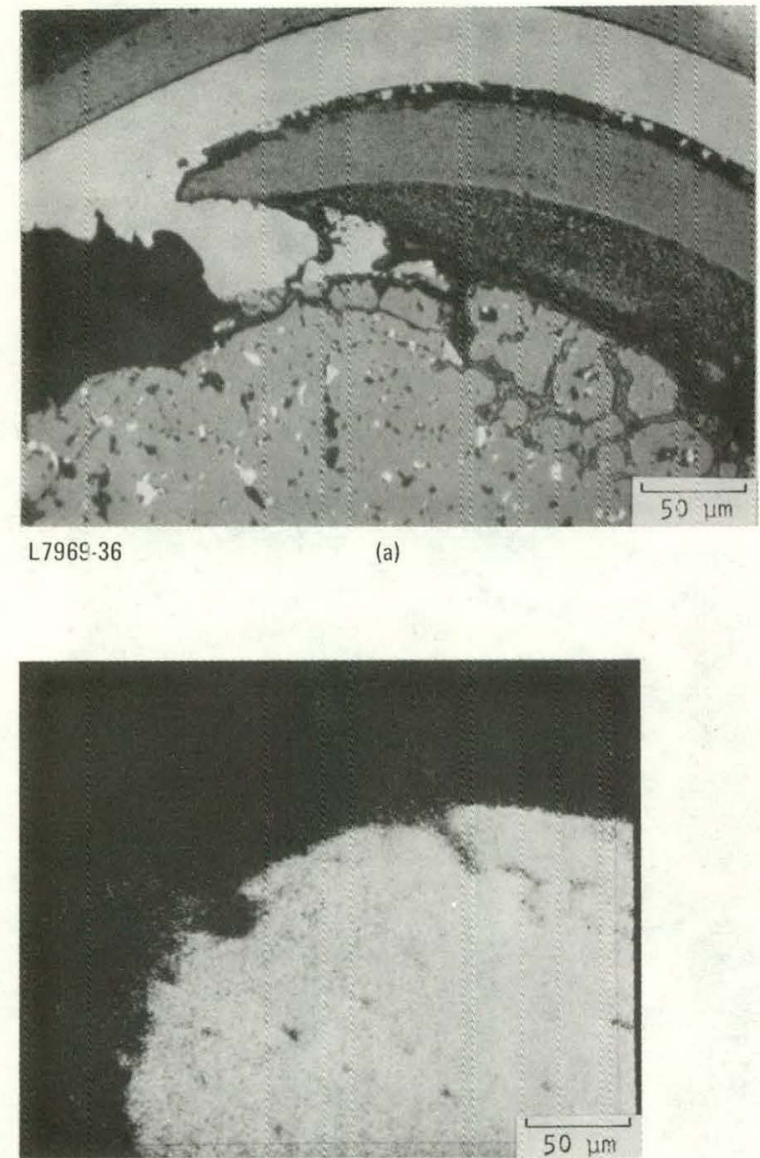

(d)

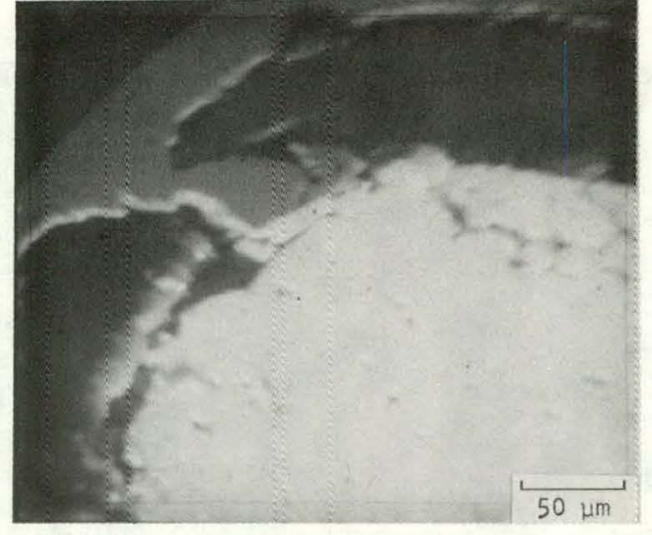

(b)

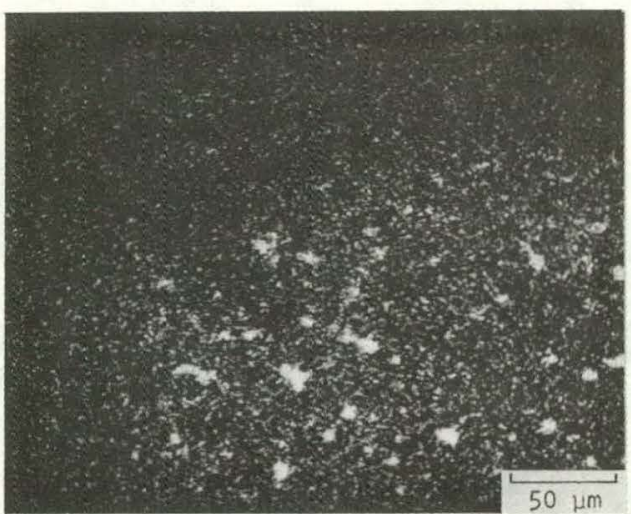

N80030-9

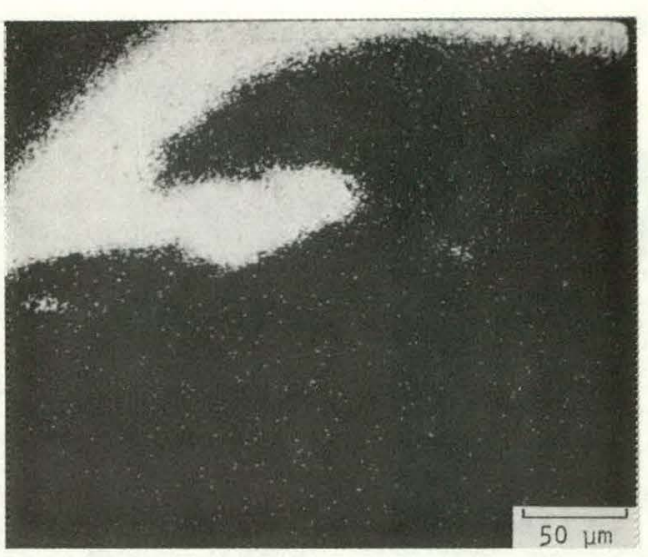

(c)

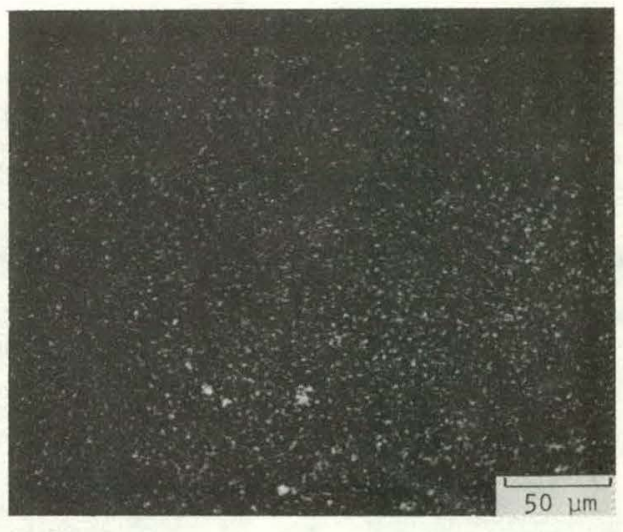

N80030-10

(f)

Fig. 5-13a. Results of electron microprobe examination of SiC corrosion of sample 17, irradiated at $1430^{\circ} \mathrm{C}$ to a burnup of $10.9 \%$ FIMA: (a) optical micrograph; (b) back-scattered electron image, (c) $\mathrm{Si} \mathrm{K \alpha}$; (d) $\mathrm{Th} \mathrm{M} \alpha$; (e) Mo L $\alpha$; (f) $\mathrm{Ru} \mathrm{L} \alpha$ 

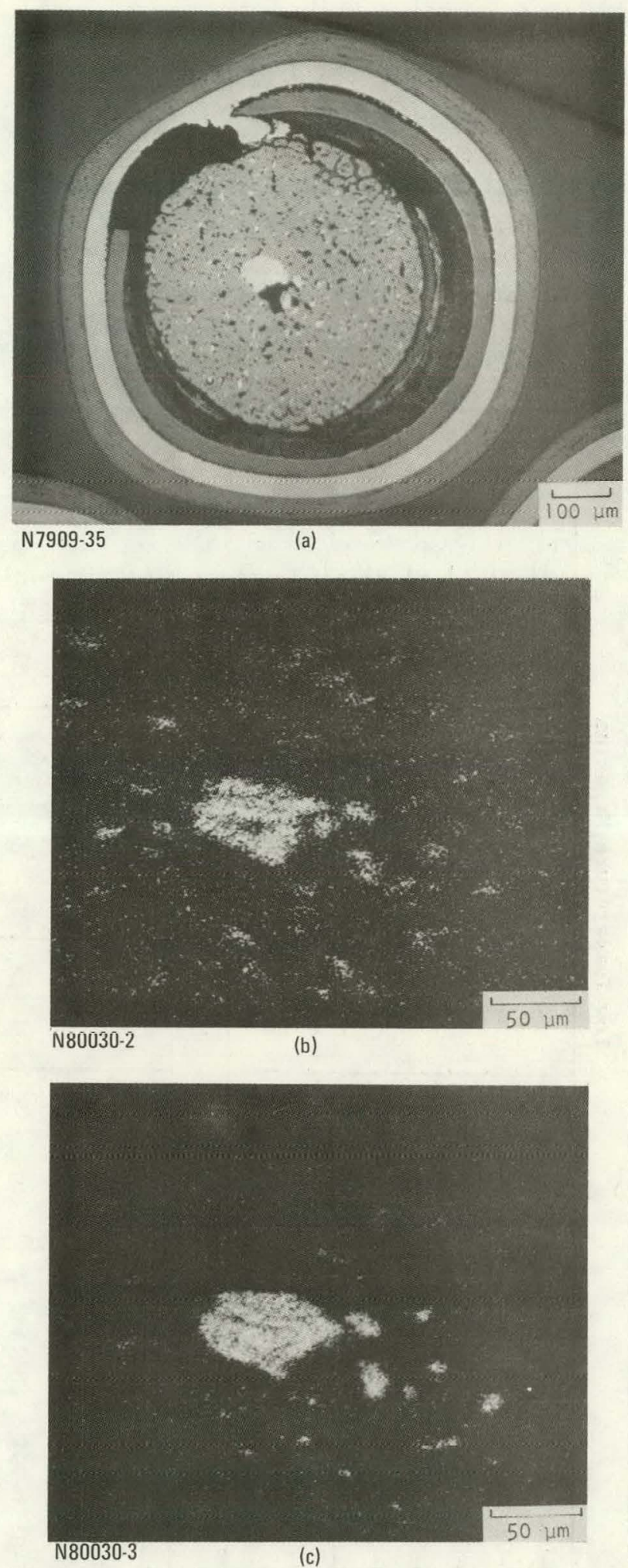

Fig. 5-13b. Results of electron microprobe of center of kernel of sample 17 irradiated at $1430^{\circ} \mathrm{C}$ to a burnup of $10.9 \%$ FIMA: (a) optical micrograph; (b) Mo $L \alpha$; (c) $\mathrm{Ru} L \alpha$ 
TABLE 5-1

RESULTS OF VISUAL EXAMIISATION

\begin{tabular}{|c|c|c|c|c|c|c|c|c|c|c|}
\hline \multirow{2}{*}{$\begin{array}{l}\text { Cap- } \\
\text { sule } \\
\text { Posi- } \\
\text { tion }\end{array}$} & \multirow[b]{2}{*}{$\begin{array}{l}\text { Particle Batch } \\
\text { Number (6252-) }\end{array}$} & \multirow{2}{*}{$\begin{array}{l}\text { Time- } \\
\text { Averaged } \\
\text { Tempera- } \\
\text { ture }(\mathrm{a}) \\
\left({ }^{\circ} \mathrm{C}\right)\end{array}$} & \multirow{2}{*}{$\begin{array}{l}\text { Fluence } \\
{\left[x \quad 10^{25} \mathrm{n} / \mathrm{m}^{2}\right.} \\
(\mathrm{E}>29 \\
\left.\text { fJ) }{ }_{\text {HTGR }}\right]\end{array}$} & \multirow[b]{2}{*}{$\begin{array}{l}\text { Burnup } \\
(\% \text { FIMA })\end{array}$} & \multirow{2}{*}{$\begin{array}{l}\text { Number of } \\
\text { Particles } \\
\text { Examined }\end{array}$} & \multicolumn{2}{|c|}{$\begin{array}{l}\text { OPyC Coating Failure } \\
(\%)\end{array}$} & \multicolumn{2}{|c|}{$\begin{array}{l}\text { Total Particle } \\
\text { Failure }(\%)\end{array}$} & \multirow{2}{*}{$\mid \begin{array}{c}\text { Predicted } \\
\text { Particle } \\
\text { Failure } \\
(\%)\end{array}$} \\
\hline & & & & & & Mean & $\begin{array}{l}95 \% \text { Confidence } \\
\text { Limit P }\end{array}$ & Mean & $\begin{array}{l}95 \% \text { Confidence } \\
\text { Limit P }\end{array}$ & \\
\hline
\end{tabular}

Low Temperature Magazine

\begin{tabular}{|c|c|c|c|c|c|c|c|c|c|c|}
\hline 2. & $20-0161-001$ & 1180 & 5.1 & 5.1 & 56 & 0 & $0 \leq \mathrm{P} \leq 5.1$ & 0 & $0 \leq \mathrm{P} \leq 5.1$ & 0 \\
\hline 4 & $07-0262-001$ & 1180 & 5.8 & 5.7 & 57 & 0 & $0 \leq \mathrm{P} \leq 5.1$ & 0 & $0 \leq \mathrm{P} \leq 5.1$ & 0 \\
\hline 5 & $14-0261-001$ & 1210 & 6.1 & 6.0 & 56 & 8.9 & $4.3 \leq P \leq 18$ & 0 & $0 \leq P \leq 5.1$ & 59 \\
\hline 7 & $14-0171-001$ & 1230 & 6.7 & 6.7 & 56 & 0 & $0 \leq \mathrm{P} \leq 5.1$ & 0 & $0 \leq \mathrm{P} \leq 5.1$ & 51 \\
\hline 8 & $13-0161-001$ & 1220 & 7.0 & 7.0 & 57 & 0 & $0 \leq P \leq 5.1$ & 0 & $0 \leq P \leq 5.1$ & 0 \\
\hline 10 & $14-0161-001$ & 1240 & 7.5 & 7.6 & 57 & 1.8 & $0.2 \leq P \leq 8.0$ & 0 & $0 \leq P \leq 5.1$ & 0 \\
\hline 11 & $15-0161-001$ & 1250 & 7.7 & 7.9 & $57^{(\mathrm{b})}$ & 1.8 & $0.2 \leq P \leq 3.0$ & 0 & $0 \leq P \leq 5.1$ & 0 \\
\hline 13 & $16-0161-001$ & 1180 & 8.2 & 8.5 & 56 & 0 & $0 \leq \mathrm{P} \leq 5.1$ & 0 & $0 \leq P \leq 5.1$ & 0 \\
\hline
\end{tabular}

High Temperature Magazine

\begin{tabular}{|c|c|c|c|c|c|c|c|c|c|c|}
\hline 15 & $20-0161-002$ & 1430 & 9.2 & 10.5 & $30^{(d)}$ & $6.2^{(c)}$ & $3.2 \leq P \leq 13$ & 6.2 & $3.2 \leq \mathrm{P} \leq 13$ & 0 \\
\hline 17 & $07-0262-002$ & 1430 & 9.4 & $10.9^{-}$ & $81^{(d)}$ & $36^{(c)}$ & $28 \leq \mathrm{P} \leq 45$ & 36 & $28 \leq \mathrm{P} \leq 45$ & 94 \\
\hline 18 & $14-0161-002$ & 1440 & 9.5 & 11.2 & $81^{(d)}$ & (e) & $(e)$ & (e) & $(e)$ & 90 \\
\hline 20 & $14-0271-001$ & 1440 & 9.7 & 11.6 & $83^{(d)}$ & $71^{(c)}$ & $57 \leq P \leq 37$ & 71 & $57 \leq P \leq 87$ & 11 \\
\hline 21 & $14-0181-001$ & 1440 & 9.8 & 11.9 & $82^{(d)}$ & $79^{(c)}$ & $64 \leq P \leq 94$ & 78 & $64 \leq P \leq 94$ & 12 \\
\hline 23 & $17-0161-001$ & 1460 & 10.0 & 12.2 & $81^{(d)}$ & (e) & (e) & (e) & (e) & 9 \\
\hline 24 & $15-0171-001$ & 1450 & 10.1 & 12.4 & $81^{(d)}$ & (e) & (e) & (e) & (e) & 4 \\
\hline 26 & $13-0171-001$ & 1440 & 10.2 & 12.7 & $85^{(d)}$ & $19^{(\mathrm{c})}$ & $1.3 \leq P \leq 27$ & 19 & $13 \leq P \leq 27$ & 36 \\
\hline
\end{tabular}

(a) Maximum surface temperature of particles

(b) Original sample size was 56

(c) OPyC coating failure was always observed with total particle failure

(d) Original sample sizes given because could not determine total number particles examined

(e) Could not determine due to very high coating failure (estimated to be $>80 \%$ ) 
RESULTS OF METALLOGRAPHIC EXAYIiATION

\begin{tabular}{|c|c|c|c|c|c|c|c|c|c|c|c|c|c|c|}
\hline \multirow{4}{*}{$\begin{array}{l}\text { Cap- } \\
\text { sule } \\
\text { Posi- } \\
\text { tion }\end{array}$} & \multirow{4}{*}{$\begin{array}{l}\text { Particle Batch } \\
\text { ilumber }(6252-)\end{array}$} & \multirow{3}{*}{\multicolumn{2}{|c|}{$\begin{array}{c}\text { Time- } \\
\text { Averaged } \\
\text { Temperature } \\
\left.\square^{\circ} \mathrm{C}\right)\end{array}$}} & \multirow{4}{*}{$\begin{array}{c}\text { Fluence } \\
{\left[x \quad 10^{25} \mathrm{n} / \mathrm{m}^{2}\right.} \\
(\mathrm{E}>29 \\
\left.\mathrm{fJ}){ }_{\mathrm{HTGR}}\right]\end{array}$} & \multirow{4}{*}{$\begin{array}{l}\text { Burnup } \\
\text { (\% FIMA) }\end{array}$} & \multirow{4}{*}{$\begin{array}{l}\text { Total } \\
\text { Sample }\end{array}$} & \multicolumn{7}{|c|}{ Number of Observations } & \multirow{4}{*}{$\begin{array}{c}\text { OPyC } \\
\text { Coating } \\
\text { Failed }\end{array}$} \\
\hline & & & & & & & \multirow{3}{*}{$\begin{array}{l}\text { Kernel } \\
\text { Migra- } \\
\text { tion }\end{array}$} & \multicolumn{2}{|c|}{ IPyC Coating } & \multicolumn{4}{|c|}{ SiC Coating } & \\
\hline & & & & & & & & & & & Local- & \multirow[b]{2}{*}{ Porosity } & \multirow{2}{*}{$\begin{array}{l}\text { Circum- } \\
\text { ferential } \\
\text { Fracture }\end{array}$} & \\
\hline & & Kerne: & Surface & & & & & Failed & Debonded & Failed & Attack & & & \\
\hline 5 & $14-0261-001$ & -1240 & 1210 & 6.1 & 6.0 & 16 & 0 & 9 & 10 & 0 & 0 & 0 & 0 & 0 \\
\hline 10 & $14-0161-001$ & 1280 & 1240 & 7.5 & 7.6 & 15 & 0 & 8 & 11 & 0 & ${ }_{1}^{(a)}$ & 8 & 2 & 0 \\
\hline 11 & $15-0161-001$ & 1300 & 1250 & 7.7 & 7.9 & 15 & 0 & 5 & 7 & 0 & $2^{\text {(a) }}$ & 13 & 1 & 0 \\
\hline 13 & $16-0161-001$ & 1230 & 1130 & 8.2 & 8.5 & 15 & 0 & 10 & 12 & 0 & $2^{(a)}$ & 12 & 6 & 0 \\
\hline 15 & $20-0161-002$ & 1490 & 1430 & 9.2 & 10.5 & 15 & 5 & N.D. & is.d. & 8 & $7^{\text {(b) }}$ & 0 & 0 & 2 \\
\hline 17 & $07-0262-002$ & 1490 & 1430 & 9.4 & 10.9 & 15 & 13 & 13 & 4 & 8 & $14^{(b)}$ & 0 & 0 & 1 \\
\hline
\end{tabular}

(a) Penetration was: No. $10-18 \mu \mathrm{m}$; No. $11-9 \mu \mathrm{m}$ (no measurement for other particles); and No. $13-10$ and $9 \mu \mathrm{m}$.

(b) SiC attack had different cause and appearance than lower temperature samples. 
THIS PAGE

\section{WAS INTENTIONALLY LEFT BLANK}


RESULTS OF $Y$-COATINGG(a) OF HT-34 SARPLES

\begin{tabular}{|c|c|c|c|c|c|c|c|c|c|c|c|c|c|c|c|c|c|c|c|c|c|c|c|c|c|}
\hline \multirow[b]{4}{*}{$\begin{array}{l}\text { Caspule } \\
\text { Position No. }\end{array}$} & \multirow{4}{*}{$\begin{array}{l}\text { Nor. of } \\
\text { partcles } \\
\text { counted }\end{array}$} & \multicolumn{24}{|c|}{ 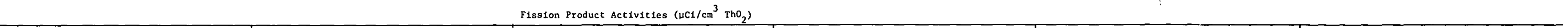 } \\
\hline & & \multicolumn{4}{|c|}{$\frac{2 \mathrm{x}-95}{2(724 \mathrm{Kev})}$} & \multicolumn{4}{|c|}{$\underset{\text { Measured }}{\mathrm{Cs}-134(605 \mathrm{Kev})}$} & \multirow{2}{*}{\multicolumn{2}{|c|}{${ }_{\text {Measured }}^{c \text { cs-137 }}$}} & \multirow[b]{3}{*}{ 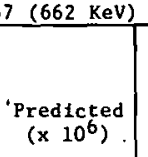 } & \multirow[b]{3}{*}{$\left|\begin{array}{c}\text { Difference (b) } \\
(\xi)\end{array}\right|$} & \multirow{2}{*}{\multicolumn{2}{|c|}{${ }_{\text {Measured }}^{\text {Ce-14 }}$}} & \multirow[b]{3}{*}{ 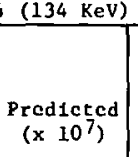 } & \multirow[b]{3}{*}{$\begin{array}{c}\text { Dif ference (b) } \\
(z)\end{array}$} & \multicolumn{4}{|c|}{ Pa-233 (312 Kev) } & \multicolumn{4}{|c|}{ Ru-106 (512 Kev) } \\
\hline & & & sured & & & Meą & sured & & & & & & & & & & & $\frac{\text { Measi }}{1}$ & sured & & & Meat & sured & & \\
\hline & & 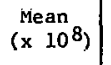 & $\begin{array}{l}\text { Deviation } \\
(z) \\
\text { Den }\end{array}$ & $\left|\begin{array}{ccc}\text { Predicted } \\
(x & \left.10^{8}\right)\end{array}\right|$ & 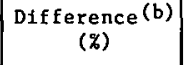 & 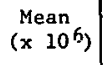 & $\begin{array}{c}\text { (z) } \\
\text { Devation }\end{array}$ & 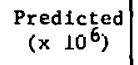 & $\mid \begin{array}{l}\text { Differencee } \\
(\xi)\end{array}$ & 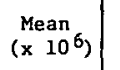 & $\begin{array}{l}\text { Devation } \\
\text { (६) }\end{array}$ & & & $\mid \begin{array}{l}\text { Mean } 7 \\
\left(x<10^{7}\right)\end{array}$ & 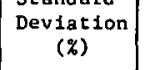 & & & Metain & 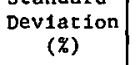 & $\left.\begin{array}{c}\text { Predicted } \\
(x 109)\end{array}\right]$ & $\mid \begin{array}{c}\text { Difference } \\
(\mathcal{2})\end{array}$ & $\left.\begin{array}{l}\text { Mean } \\
\left(x 0^{6}\right)\end{array}\right]$ & $\left|\begin{array}{c}\text { Deviar ion } \\
(\xi)\end{array}\right|$ & $\left|\begin{array}{c}\text { Predicted } \\
(x 106)\end{array}\right|$ & $\mid \begin{array}{c}\text { Difference(b) } \\
(\text { (n) }\end{array}$ \\
\hline 2 & 54 & 2.26 & 13 & 1.58 & -30.1 & 2.33 & 13 & 2.07 & -11.1 & 2.16 & 13 & 1.50 & -30.6 & 3.98 & 13 & 3.68 & -7.5 & \begin{tabular}{l|l}
4.97 &
\end{tabular} & 14 & 4.17 & -16.1 & ND & -- & $\cdots$ & $\cdots$ \\
\hline 4 & 56 & 2.26 & 14 & 1.78 & -21.2 & 2.49 & 14 & 2.40 & -3.6 & 2.17 & 14 & 1.69 & -22.1 & 4.03 & 14 & 4.15 & -3.0 & 4.79 & 14 & 4.45 & -7.1 & ND & -- & -- & -- \\
\hline 5 & 51 & 2.64 & 14 & 1.87 & -29.2 & 2.96 & 15 & 2.56 & -13.5 & 2.54 & 15 & 1.78 & -29.9 & 4.71 & 14 & 4.39 & -7.0 & 5.52 & 14 & 4.58 & -17.0 & ND & -- & --- & -- \\
\hline 7 & 38 & 2.54 & 5.4 & 2.06 & -18.9 & 3.21 & 6.5 & 2.86 & -10.9 & 2.56 & 6.2 & 1.97 & -23.0 & 4.56 & 4.7 & 4.86 & 6.6 & 4.98 & 5.6 & 4.83 & -3.0 & 2.61 & 7.4 & 2.28 & -12.6 \\
\hline 8 & 56 & 2.65 & 4.5 & 2.15 & -18.9 & 3.21 & 13 & 3.00 & -6.5 & 2.44 & 13 & 2.06 & -15.6 & 4.72 & 3.9 & 5.08 & 7.6 & 4.44 & 4.8 & 4.94 & 11.3 & 2.67 & 7.9 & 2.39 & -10.5 \\
\hline 10 & 53 & 2.97 & 20 & 2.34 & -11.7 & 3.80 & 20 & 3.30 & -13.2 & 2.87 & 20 & 2.24 & -21.9 & 5.31 & 19 & 5.54 & 4.3 & 5.56 & 20 & 5.15 & -7.4 & 3.22 & 20 & 2.62 & -18.6 \\
\hline 11 & 53 & 3.14 & 26 & 2.44 & -22.3 & 4.03 & 18 & 3.44 & -14.6 & 2.96 & 19 & 2.33 & -21.3 & 5.58 & 27 & 5.78 & 3.6 & 5.70 & 25 & 5.25 & -7.9 & 3.33 & 24 & 2.75 & -17.4 \\
\hline 13 & 55 & 3.17 & 3.4 & 2.61 & -17.7 & 4.44 & 3.3 & 3.70 & -16.7 & 3.10 & 3.4 & 2.50 & -19.3 & 5.75 & 3.2 & 6.22 & 8.2 & 5.67 & 3.3 & 5.43 & -4.2 & 3.40 & 5.5 & 2.97 & -12.6 \\
\hline 15 & 68 & 4.13 & 20 & 3.18 & -23.0 & 0.28 & 301 & 4.48 & 1500 & 0.49 & 127 & 3.06 & 524 & 4.00 & 72 & 7.64 & 91.0 & ND & -- & -.- & ND & 4.54 & 19 & 3.73 & -17.8 \\
\hline 17 & 49 & 4.13 & 2.5 & 3.32 & -19.6 & 0.60 & 258 & 4.66 & 677 & 0.73 & 137 & 3.20 & 384 & 5.75 & 38 & 7.99 & 39.0. & 6.69 & 2.8 & 6.03 & -9.9 & ND & --- & --- & -- \\
\hline 20 & 22 & 4.00 & 33 & 3.52 & -12.0 & 0.08 & 78 & $\begin{array}{l}4.92 \\
.5 .2\end{array}$ & 6050 & 0.46 & 48 & 3.40 & 639 & 5.61 & 62 & 8.51 & 51.7 & 6.18 & 33 & $6.18^{\circ}$ & 0 & ND & -- & -- & -- \\
\hline 26 & 66 & 4.50 & 15 & 3.83 & -14.9 & 0.66 & 279 & 5.27 & 698 & 0.73 & 150 & 3.69 & 405. & 5.62 & 56 & 9.28 & 65.1 & 6.97 & 15 . & 6.37 & -8.6 & ND & $\cdots$ & $\cdots$ & $\cdots$ \\
\hline
\end{tabular}

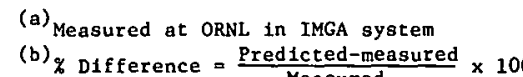

Note:
ND
Not 
Blark 
TABLE 5-4
FISSION FRODUCT RATIOS(a)

\begin{tabular}{|c|c|c|c|c|c|c|c|c|c|c|c|c|c|c|c|c|c|c|c|c|c|c|}
\hline \multirow[b]{3}{*}{, } & \multirow[b]{3}{*}{$\begin{array}{l}\text { Posi- } \\
\text { tion }\end{array}$} & \multirow[b]{3}{*}{$\begin{array}{c}\text { Number } \\
\text { of } \\
\text { Part1- } \\
\text { cles }\end{array}$} & \multicolumn{4}{|c|}{ Cs $-134 / 2 t-95$} & \multicolumn{4}{|c|}{ Cs-137/Zr-95 } & \multicolumn{4}{|c|}{ Cs-1 37/Ru-106 } & \multicolumn{4}{|c|}{ Cs-134/Cs-137 } & \multicolumn{4}{|c|}{$\mathrm{Ce}-144 / 2 \mathrm{r}-95$} \\
\hline & & & \multicolumn{2}{|c|}{ Measured } & \multirow[b]{2}{*}{$\begin{array}{c}\text { Pre- } \\
\text { dicted } \\
\text { Value }\end{array}$} & \multirow[b]{2}{*}{$\left|\begin{array}{c}\operatorname{Rat10}_{(\text {(b) }}^{(b)} \\
\mid\end{array}\right|$} & \multicolumn{2}{|c|}{ Measured } & \multirow[b]{2}{*}{$\begin{array}{l}\text { Pre- } \\
\text { dicted } \\
\text { Value }\end{array}$} & \multirow[b]{2}{*}{$\operatorname{Rat}_{(\mathrm{z})}^{\text {Ra) }}$} & \multicolumn{2}{|c|}{ Measured } & \multirow[b]{2}{*}{$\begin{array}{c}\text { Pre- } \\
\text { dicted } \\
\text { Value }\end{array}$} & \multirow[b]{2}{*}{$\begin{array}{c}\operatorname{Ratrio}_{(\mathrm{b})} \\
(\tilde{i} ;\end{array}$} & \multicolumn{2}{|c|}{ Measured } & \multirow[b]{2}{*}{$\begin{array}{l}\text { Pre- } \\
\text { dicted } \\
\text { Value }\end{array}$} & \multirow[b]{2}{*}{$\operatorname{Rat10}_{(\mathrm{K})}^{(\mathrm{b})}$} & \multicolumn{2}{|c|}{ :easured } & \multirow[b]{2}{*}{$\begin{array}{l}\text { Pre- } \\
\text { dicted } \\
\text { Value }\end{array}$} & \multirow[b]{2}{*}{$\underset{(\%)}{\operatorname{Ratio}^{(b}}$} \\
\hline & & & Mean & $\begin{array}{c}\text { Stan- } \\
\text { dand } \\
\text { Dewi- } \\
\text { atfion } \\
(\Sigma)\end{array}$ & & & Man & $\begin{array}{c}\text { Stan- } \\
\text { dard } \\
\text { Devi- } \\
\text { ation } \\
(\mathrm{g})\end{array}$ & & & Mean & $\begin{array}{c}\text { Stan- } \\
\text { dard } \\
\text { Devi- } \\
\text { atton } \\
(x) \\
\end{array}$ & & & Mean & $\begin{array}{c}\text { Stan- } \\
\text { dard } \\
\text { Devi- } \\
\text { ation } \\
(\%)\end{array}$ & & & Mean & $\begin{array}{l}\text { Stan- } \\
\text { dard } \\
\text { Devi- } \\
\text { ation } \\
\text { (\%) }\end{array}$ & & \\
\hline & & & $\left(\begin{array}{ll}x & 10^{-2}\end{array}\right)$ & & $\left(x 1^{-2}\right.$ & & $\left(x 1^{-3}\right)$ & & $\left(\times 10^{-3}\right)$ & & $\left(\times 10^{-1}\right)$ & & $\left(\begin{array}{l}\times 10^{-1}\end{array}\right)$ & & $\left(x \quad 10^{0}\right)$ & & $\left(x \quad 10^{0}\right)$ & & ( $\times 10^{-1}$ ) & & $\left(x 1^{-1}\right)$ & \\
\hline & 2 & 54 & 1.03 & 0.98 & 1.32 & 78.0 & 9.62 & 1.03 & 9.50 & 101.3 & ND & -- & -- & -- & 1.07 & 1.24 & 1.39 & 77.0 & 1.77 & 1.05 & 2.32 & 76.3 \\
\hline & 4 & 56 & 1.16 & $\cdot .23$ & 1.35 & 81.5. & $\varsigma .63$ & 1.32 & 9.52 & 101.1 & ND & -- & -- & -- & 1.14 & 1.64 & 1.42 & 80.3 & 1.78 & 0.68 & 2.34 & 76.1 \\
\hline & 5 & 51 & 1.12 & 4.20 & 1.37 & 81.7 & $\subseteq .56$ & 3.40 & 9.53 & 100.3 & ND & -- & -- & -- & 1.17 & 1.79 & 1.43 & 81.8 & 1.78 & 1.17 & 2.35 & 75.7 \\
\hline & 7 & 38 & $i .2 E$ & 3.86 & 1.39 & 90.6 & 16.00 & 3.71 & 9.55 & 104.7 & 9.86 & 6.23 & 8.64 & 114.1. & 1.25 & 2.49 & 1.46 & 85.6 & 1.79 & 0.94 & 2.35 & 74.0 \\
\hline & 8 & 56 & $i .21$ & $1: .14$ & 1.43 & 86.4 & $\subseteq 20$ & 12.66 & 9.56 & 96.2 & 9.13 & 13.58 & .8 .60 & 106.2 & 1.32 & 7.28 & 1.46 & 90.4 & 1.78 & 1.27 & 2.36 & 75.1 \\
\hline & 10 & 53 & 1.28 & 2.58 & 1.41 & 90.3 & 9.66 & 2.23 & 9.57 & 100.9 & 8.93 & 5.82 & 3.53 & $10<.7$ & 1.32 & 1.45 & 1.47 & 89.8 & ND & ND & 2.37 & ND \\
\hline & 11 & 53 & 1.3 & 5.94 & 1.42 & 92.2 & 9.61 & 4.77 & 9.58 & 100.3 & 8.91 & 5.96 & 8.49 & 104.9 & 1.37 & 2.12 & 1.48 & 92.6 & 1.80 & 0.76 & 2.37 & 75.9 \\
\hline & 13 & 55 & 1.40 & 1.34 & 1.41 & 99.3 & 9.74 & 1.27 & 9.59 & 101.6 & 9.11 & 4.94 & 8.42 & 108.2 & 1.43 & 1.21 & 1.48 & 96.6 & 1.81 & $0.93^{\circ}$ & 2.38 & 76.4 \\
\hline & 15 & 68 & 0.056 & 323 & 1.41 & 4.7 & 1.20 & 130 & 9.61 & .12 .5 & 1.10 & 127 & 8.20 & 13.4 & 0.28 & 84.83 & 1.46 & 19.3 & 0.965 & 70.80 & 2.40 & 40.2 \\
\hline & 17 & 49 & 0.145 & $2 \times 0$ & 1.41 & $10.3^{\circ}$ & 1.77 & 136 & 9.62 & 18.4 & ND & -- & -- & -- & 0.32 & 122 & 1.46 & 22.3 & 1.39 & 38.5 & 2.41 & 57.7 \\
\hline & 20 & 22 & 0.0191 & 73.78 & 1.39 & 1.4 & 1.08 & 36.077 & 9.63 & 11.2 & ND & -- & -- & -- & 0.17 & 65.30 & 1.45 & 11.7 & 1.33 & 51.40 & 2.42 & 55.0 \\
\hline & 26 & 66 & 0.143 & 284 & 1.38 & 10.5 & 1.61 & 151 & 9.65 & 16.7 & ND & -- & -- & -- & 0.25 & 196 & 1.43 & 17.6 & 1.28 & 52.18 & 2.42 & 52.9 \\
\hline
\end{tabular}

(a) leasured at ORYL in IrIGe system

(b) Rat1o $=\frac{\text { Measured }}{\text { Predicted }} \times 100$ 
TABLE 5-5

TRIGA FISSION GAS RELEASE DATA AND CALCULATED PARTICLE FAILURE

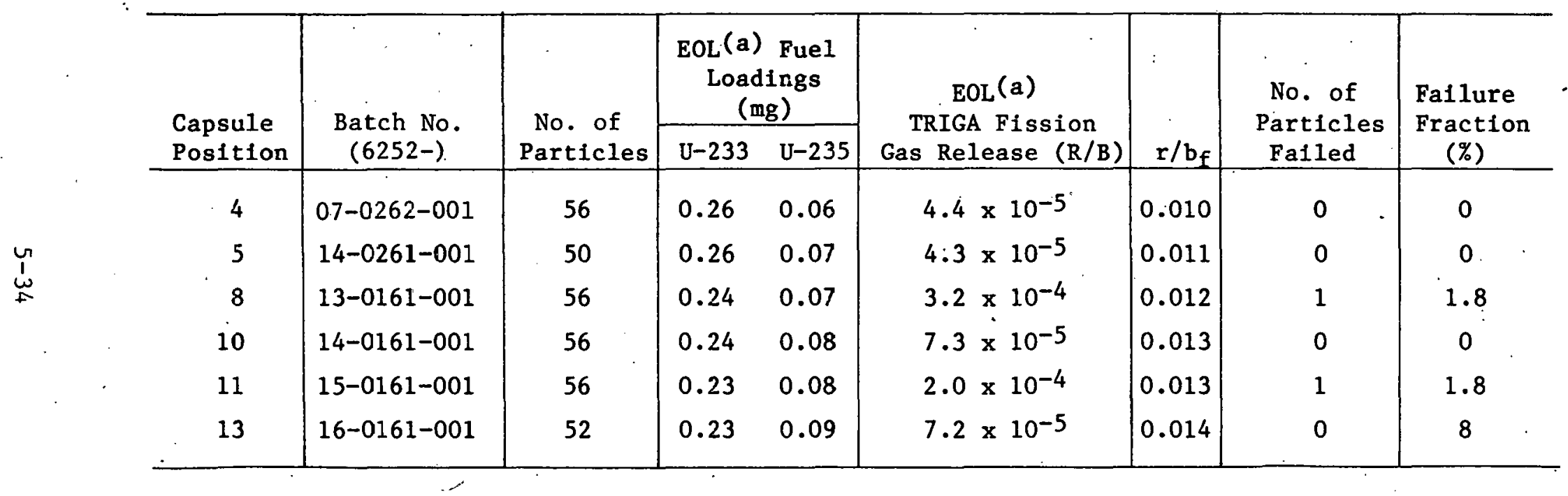

(a) End-of-Life 


\section{DISCUSSION}

\subsection{FISSION PRODUCT RELEASE}

Fission product activities were measured in the particles of all the low-temperature and four high-temperature samples. The average measured and predicted activities (see Table 5-3) of each sample are plotted in Fig. 6-1. The activities were divided by the kernel volume to account for the differences in kernel sizes. The figure shows that the difference in measured and predicted values is relatively constant for the low-temperature samples. This difference is caused by uncertainties in the measured and predicted activities.

Zirconium is known to form a stable oxide. Therefore, in an oxide kernel, the $\mathrm{Zr}$ should remain in the kernel, as verified by the $\mathrm{HT}-34$ results. The measured $\mathrm{Zr}$ activity was, on the average, $20 \%$ higher than the predicted value. This difference was relatively constant for both the low- and hightemperature samples. Figure 6-1 shows that $\mathrm{Ru}$ and $\mathrm{Pa}$ also remained in the kernel at both temperatures. However, the measured activities of Ce and Cs were significantly lower than the predicted values for the high-temperature samples. Therefore, $\mathrm{Ce}$ and $\mathrm{Cs}$ were released from the particles in those samples.

Various fission product ratios were calculated for the samples and are given in Table 5-4. Since $\mathrm{Zr}$ remained in the kernel even though the particle failed, this isotope could be compared to the more volatile isotopes to indicate fission product release and particle failure. Table 5-4 compares the measured fission product ratios, such as $\mathrm{Cs} / \mathrm{Zr}$, to the predicted ratios. The table shows that $C s$ and Ce were lost from the high-temperature samples. An average of $32 \% \mathrm{Ce}$ was lost from the particles irradiated at $\sim 1435^{\circ} \mathrm{C}$ based on comparing the low- and high-temperature $\mathrm{Ce} / \mathrm{Zr}$ measured and predicted ratios. 
The ratio of $\mathrm{Cs}-137$ to $\mathrm{Zr}-95$ was selected to evaluate in detail the particle failure of the capsule HT-34 samples. After studying the distributions of the $\mathrm{Cs} / \mathrm{Zr}$ ratios (see Fig. 5-11), it was observed that the ratios were either relatively normally distributed or skewed to the lower $\mathrm{Cs} / \mathrm{Zr}$ ratios. For example, sample 13 was normally distributed, and sample 11 was skewed. The conclusion was that some particles lost part of their Cs inventory. The question then arose as to how to determine the number of failed particles. It seemed logical that particles that had less than a certain measured $\mathrm{Cs} / \mathrm{Zr}$ ratio were failed. The solution then was to find the lower limit of the Cs/Zr ratio of nonreleasing particles. To compare the 12 samples, the measured $\mathrm{Cs} / \mathrm{Zr}$ ratios were normalized by dividing them by the predicted Cs/Zr. This new ratio will be written as (Cs/Zr)M/P, and is reported in Table 5-4.

The means and standard deviations of the $(\mathrm{Cs} / \mathrm{Zr}) \mathrm{M} / \mathrm{P}$ ratio of the $1200^{\circ} \mathrm{C}$ samples were plotted in Fig. 6-2. The means of samples $2,4,10$, and 13 were all about $101 \%$, and the standard deviations were the smallest of the group. If there were no particle failures and predictions and the activity measurements were perfect, the ratio would be $100 \%$ for all samples. Samples 5,8 , and 11 had higher standard deviation and lower means berause some particles had apparently released Cs. The ratio for sample 7 was exceptionally high for some unknown reason. Figure 5-13 shows that the Cs/Zr ratio of samples 2, 4, 10, and 13 had approximately normal distributions. The other four had skewed distributions. The assumption was then made that the particles from samples $2,4,10$, and 13 had not released any Cs.

The next step was to determine the lowcr limit of $(\mathrm{Cs} / \mathrm{Zr}) \mathrm{M} / \mathrm{P}$ for nonrelcasing particles. Samples 2, 4, 10, and 13 were selected to be an interual standard. The mean and standard deviations of the total group of these particles were calculated to be $101.2 \%$ and $1.54 \%$ (of mean), respectively. For a large population of nonreleasing particles, $\geq 99.9 \%$ of the particles would have a ratio $295.8 \%$ at a $95 \%$ level of confidence, based on the internal standard. 
The number of particles that released $\mathrm{Cs}-137$ was then determined for the other samples. The $(\mathrm{Cs} / \mathrm{Zr}) \mathrm{M} / \mathrm{P}$ values calculated for all the particles of each sample were compared to the lower limit of ( $\mathrm{Cs} / \mathrm{Zr}$ )M/P of nonreleasing particles $(95.8 \%)$. An assumption was made that the mean of the nonreleasing group of particles of the samples that had some failed particles was the same $(101.2 \%)$ as the internal standard. Since the mean ratio should ideally be $100 \%$, all the $(\mathrm{Cs} / \mathrm{Zr}) \mathrm{M} / \mathrm{P}$ ratios were then normalized to $100 \%$. The lower limit of the $(\mathrm{Cs} / \mathrm{Zr}) \mathrm{M} / \mathrm{P}$ of the nonreleasing particles was also normalized $(94.6 \%)$. The range of ( $\mathrm{Cs} / \mathrm{Zr}) \mathrm{M} / \mathrm{P}$ ratios of each sample is plotted in Fig. 6-3. The number of particles above and below the normalized lower limit is given. The particles above the line were assumed to have retained all their Cs. The particles below the line lost part of their Cs inventory.

The results of the fission product failure analysis are summarized in Table 6-1. Six percent to $21 \%$ of the particles in four of the lowtemperature samples released Cs. The SiC coating had failed in these particles. The SiC coating was designed to fail mechanically for samples 5 and 7 (thin buffer coatings). The SiC failure in samples 8 and 11 was not expected. The metallographic examination of sample 11 (sample 8 not examined) showed a fine porosity in the SiC coating. If the pores were interconnected, Cs may have migrated through the SiC coating in samples 8 and 11 . Most of the OPyC coatings were visibly intact (examined at $20 \mathrm{x}$ ) on samples 7, 8, and 11 (see Table 6-1). Myers (Ref. 26) calculated that the Cs could not have diffused completely through an intact pyrocarbon coating in the time the samples were irxadiated. Therefore, the Cs must have escaped through defects, possibly irradiation-induced microcracks, in the OPyC coating. The gas permeability of $\mathrm{BISO} \mathrm{ThO}_{2}$ particles irradiated in capsules P13R and P13S was partially attributed to microstructural cracking (Ref. 4). As-coated defects in the OPyC coating may have also caused the permeable coating. Since the OPYC failure was higher than the SiC coating failure for sample 5, the Cs may have escaped in particles with failed OPyC coatings. Sample 10, which was irradiated at approximately the same temperature as sample 11, apparently did not develop defects in the opyC coating. The 
metallographic examinätion indicated this sample also had porous SiC coatings, but no $\mathrm{Cs}$ was released from the particles.

The approach used to determine the particle failure appeared reasonable when the calculated lower limit $(94.6 \%)$ for $(\mathrm{Cs} / \mathrm{Zr}) \mathrm{M} / \mathrm{P}$ of nonreleasing particles was drawn on the histograms given in Fig. 5-11. For the high temperature samples $(15,17,20$, and 26$)$ the obviously failed particles were well below the lower limit line, while a few particles were above the line. These few particles had not released any $C_{s}$. Looking at the distributions of samples 5 and 11 , the point where the lower limit line divided the samples between releasing and nonreleasing particles seemed reasonable. The ratios of the failed particles were definitely separated from the ratios of the intact particles. There could be some argument that the number of failed particles determined for samples 7 and 8 may be overpredicted by one particle, because the Cs/Zr ratio of one of the failed particles is very close to the ratio of an intact particle.

Most particles in the high temperature samples released Cs. Although the SiC coating was failed, the OPyC coating had remained intact. Myers (Ref. 26) calculated that Cs could readily diffuse through the OPyC coating at the irradiation temperature of $\sim 1450^{\circ} \mathrm{C}$. The metallographic examination clearly showed the SiC coating failure was primarily caused by a chemical attack of the SiC rather than by a gas pressure buildup. Therefore, the Cs penetrated through the SiC coating and either diffused through the OPyC or migrated through defects in the OPyC coating.

The measured Cs retention by the particles was compared with predicted retention by the kernel. The predictions yielded a lower limit to the Cs retained by the particles. The true value of Cs retained by the particles would be given by the sum of (1) the Cs retained by the kernel and (2) the Cs retained by the coatings.

The calculations of the Cs retention by the kernel was based on a preliminary analysis of in-pile fission product release from the kernels of 
intact particles (Ref. 27). The Cs retained by the kernel was obtained from the relation (Ref. 26):

$$
\mathrm{fr}=1.0-2.95 \times 10^{-5} \mathrm{~F}^{2} \sqrt{\mathrm{t}} \mathrm{e}^{-4680 / \mathrm{T}} \text { for } \mathrm{fr}>0
$$

where $\quad \mathrm{fr}=$ fraction of $\mathrm{Cs}$ retained by the $\mathrm{ThO}_{2}$ kernel

$\mathrm{F}=$ burnup (\% FIMA)

$t=$ time $(s)$

$\mathrm{T}=$ temperature $(\mathrm{K})$

The predicted values of Cs retention derived from the above equation are given in Fig. 6-3 and in Table 6-2. $\Lambda$ s shown in Fig. 6-3, they are consistent, in general, with the observed values. For all sample positions shown except 15 and 17 , the mean value of the observed Cs retentions of the failed particles is approximately equal to or greater than the predicted ones; it will be recalled that the predicted values are only lower limits to the true values of Cs retention. For sample positions 5, 7, 8, and 20, where the observed mean and the predicted $C s$ retentions are similar, it is implied that all the Cs released from the kernel during the irradiation period escaped from the failed particle by the end of the irradiation.

For sample positions 15 and 17 , less Cs was retained by the kernel than predicter. Tf the predictions of $\mathrm{Cs}$ retention are correct, as an analysis of the data of HT-33 (Ref. 16) indicate, this implies an additional contribution to $C s$ release in these cases. It is known that extensive kernel migration occurred in particles in these positions (see Fig. 5-10, for example), and it is possible that this phenomenon or associated processes are the cause of the increased fission product release from the kernel (Ref. 26). However, this requires a reexamination of the results in Fig. 3-6 for sample positions 20 and 26 .

Since extensive kernel migration was observed in samples in positions 15 and 17 (see Table 5-2), similar results would be expected in positions 20 and 26. Unfortunately, such measurements were not made for the particles in 
the latter positions. Nevertheless, it is reasonable to assume extensive kernel migration for the particles in sample positions 20 and 26 and to expect, therefore, predicted Cs retentions greater than observed. While the actual predictions of $C$ s retention are smaller than observed, the predictions are based on an approximation whose validity decreases as the fractional retention decreases. Consequently, if more precise equations were used, it is likely that the predicted Cs retentions for particles of sample positions 20 and 26 would be larger than shown in Fig. 6-3 and possibly larger than the mean of the observed values for these positions (Ref. 26).

\subsection{CHEMICAL PERFORMANCE}

The chemical performance of the TRISO $\mathrm{ThO}_{2}$ particles was primarily evaluated by the metallographic examination of four $1200^{\circ} \mathrm{C}$ and two $1450^{\circ} \mathrm{C}$ samples. SiC coating reactions and kernel migration were observed. A total of 61 particles was examined.

The TRISO $\mathrm{ThO}_{2}$ particles irradiated to $1200^{\circ} \mathrm{C}$ had no kernel migration as predicted. However, unexpected corrosion of the SiC coating was seen. The microstructure of the areas of corrosion took the form of either a localized attack or a fine porosity.

The localized chemical attack of the SiC coating was observed in 5 out of 46 particles. The maximum penetration of the SiC was $18 \mu \mathrm{m}$. Palladium was detected in the reaction zone by the electron microprobe examination. Also, $\mathrm{Cl}$ was measured along the inner surface of the SiC. Palladium attack has been observed several times in TRISO fissile particles (Ref. 28). Palladium attack in TRISO $\mathrm{ThO}_{2}$ fuel has not been reported before. However, Pd attack was recently observed in the TRISO $\mathrm{ThO}_{2}$ particles irradiated in capsule HRB-14 (Ref. 29) at approximately the same temperatures as HT-34.

- The chemical attack of the SiC coating was unexpected, because the number of Pd atoms per particle was significantly less than HEU or LEU fissile particles. Table 6-3 gives the number of $\mathrm{Pd}$ atoms per particle for the 
samples examined metallographically. SiC attack was observed in three $1200^{\circ} \mathrm{C}$ samples that had 5.6 to 6.4 × $10^{14} \mathrm{Pd}$ atoms per particle formed during irradiation. SiC attack was observed in $\mathrm{HRB}-14$ TRISO $\mathrm{ThO}_{2}$ particles having $7 \times 10^{14} \mathrm{Pd}$ atoms per particle (Ref. 29); the results of both capsules are in good agreement. As a comparison of fissile and fertile particles, the $\mathrm{UO}_{2}$ particles irradiated in capsule HRB-14 had $6.4 \times 10^{15} \mathrm{Pd}$ atoms per particle. Previous experimental observations have indicated that the Pd must concentrate in local regions near the SiC coating before SiC corrosion will occur (Ref. 30). The HT-34 results support this, because the attack only had occurred when the Pd had concentrated. If the Pd concentration could be prevented, the Pd attack of the SiC coating would be minimized (Ref. 30).

The current model for predicting changes in SiC thickness due to fission product attack was revised. The predicted HT-34 corrosion SiC thinning, based on the square root of time, is given in Ref. 31. A comparison of these data and the HT-34 corrosion measurements showed that the Pd penetration in the SiC coating was higher than the upper $90 \%$ confidence level of the referenced data. The data are shown in Fig. 6-4. The poor correlation could be attributed to the uncertainties of the measurements and the temperatures.

The other type of $\mathrm{SiC}$ corrosion at $1200^{\circ} \mathrm{C}$ was the porosity around the entire SiC coating. The small pores were located radially across the SiC coating, but were concentrated near the inner surface. The porosity was not dependent upon a thermal gradient. The cause of this corrosion is uncertain. Chlorine may have contributed to the corrosion, since it was measured by electron microprobe around the inner surface of the SiC. Brown and Faircloth (Ref. 32) observed similar porosity in TRISO low-enriched $\mathrm{UO}_{2}$ irradiated at $1550^{\circ} \mathrm{C}$ to a burnup of $4 \%$ FIMA. Strontium was detected in the reaction zone. The corrosion seen in HT-34 may be batch dependent. TRISO $\mathrm{ThO}_{2}$ particles were irradiated in capsule HT-33 under the same irradiation conditions as those of HT-34. The HT-33 samples were from different production coating runs, but were made to about the same design specifications as 
5

the HT-34 samples. Two samples tested at $1200^{\circ} \mathrm{C}$ in $\mathrm{HT}-33$ were examined and no SiC corrosion was observed (Ref. 16). Porosity was observed in a different batch tested at $1420^{\circ} \mathrm{C}$ in $\mathrm{HT}-33$, but was not observed in two other batches tested at $1430^{\circ} \mathrm{C}$ in $\mathrm{HT}-34$.

SiC porosity has also been observed in an irradiated particle sample heated in an out-of-pile thermal gradient experiment (Ref. 33). The sample was from a TRISO $\mathrm{ThO}_{2}$ batch irradiated in capsule P13S. No porosity-type corrosion was seen in the SiC coating (Ref. 4) before heating. After the particles were heated for $2592 \mathrm{~h}$ at $1520^{\circ} \mathrm{C}$, porosity had formed in the SiC coating.

Corrosion of the SiC coating was observed in the two samples irradiated at $1430^{\circ} \mathrm{C}$. The corrosion apparently caused the SiC coating to fail in most particles. Similar corrosion was seen in three samples irradiated in capsules HT-31 and HT-33 (Ref. 16) at approximately the same temperatures. The corrosion occurred at the hot side of the particles. No fission products were detected in the reaction zone of the SiC coating. The SiC attack was most likely caused by either oxidation or a reaction with $\mathrm{Cl}$. Thermodynamic predictions (Ref. 35) show that oxidation at the SiC coating would occur at the hot side of the particle. Corrosion was previously noted in TRISO $\mathrm{UO}_{2}$ particles irradiated at $1250^{\circ}$ and $1500^{\circ} \mathrm{C}$ and was hypothesized to be caused by oxidation ( $\operatorname{Ref}, 36$ ). However, a chemical reaction with $\mathrm{Cl}$ may have occurred instead. In a thermal-gradient annealing experiment at $1360^{\circ}$ and $1440^{\circ} \mathrm{C}$, SiC corrosion of unirradiated $\mathrm{UC}_{2}$ particles had occurred at the hot side of the particles. The corrosion was attributed to $\mathrm{Cl}$ attack (Ref. 37). It should be noted that no Pd attack was observed in the HT -34 particles irradiated at $1430^{\circ} \mathrm{C}$.

As expected, kernel migration had occurred. The maximum migration distance was measured to be $60 \mu \mathrm{m}$ for sample 17 . The kernel migration coefficient (KMC) was calculated to be $4.8 \times 10^{-10}\left(\mathrm{~m}^{2} \mathrm{x} \mathrm{k} / \mathrm{s}\right)$ at an average kernel temperature of $1490^{\circ} \mathrm{C}$. This $\mathrm{KMC}$ value falls within the predicted range for the KMC at the $90 \%$ confidence level (Ref. 34). 


\subsection{MECHANICALL PERFORMANCE}

The mechanical performance of the $1200^{\circ} \mathrm{C}$ samples was evaluated by characterizing the pressure vessel and OPyC coating failure. A summary of the failure of the TRISO $\mathrm{ThO}_{2}$ particles irradiated in HT-34 is given in Table 6-1. The mechanical performance of the $1450^{\circ} \mathrm{C}$ samples will not be discussed because of the high SiC failure caused by chemical corrosion.

Visual and metallographic examinations and fission gas and fission product release measurements were used to evaluate coating failure for the HT-34 samples. None of the measurement methods may have given an accurate measure of the SiC coating failure, because the OPyC coating remained intact on most particles. The best indication of the SiC failure was determined by the Cs release measurements.

The most obvious result was that the pressure-vessel performance model over-predicted the failure of the two thin buffer coatings (samples 5 and 7 ). Figure 6-3 shows that 6 out of 89 particles (7\%) were failed. For an infinite population, this failure would be $\leq 12 \%$ at $95 \%$ confidence. The predicted failure was $\sim 55 \%$. These results imply that there is considerable margin within the existing TRISO $\mathrm{ThO}_{2}$ designs for increasing heavy metal fue1 density.

As discussed in Section 3.1.1, the frequency of flaws was varied for samples 5 and 7 . Increasing the flaw frequency from $9 \%$ to $29 \%$ did not increase the SiC failure, and therefore did not apparently affect the particle performance.

Although no pressure-vessel failure was predicted for the other six low-temperature samples, SiC failure was observed in two of the samples (see Table 6-1). However, the loss of integrity was apparently a chemical rather than a mechanical failure, as discussed in section 6.1. The visual examination indicated zero pressure-vessel failure. It is concluded that no 
pressure-vessel failure occurred in the particles; this is in good agreement with the model predictions.

The FGR measurement on sample 8 indicated that at least one particle failed, even though the OPyC coatings were all visibly intact. This result suggests that the OPYC coating had defects that allowed $\mathrm{Kr}$ to escape, which is consistent with the fission product release analysis discussed in Section 6.1 .

The failed HT-34 particles, based on Cs release, were not visibly cracked, but rather were permeable to metallic and gaseous fission products. No data presently exist to predict the fission gas released from a permeable TRISO particle: Therefore, the FGR data cannot be used to determine accurately the particle failure fractions.

The OPyC coating failure was measured by a visual examination at $4 \mathrm{x}$ and 20x. Five samples had zero failure, and three had $1.8 \%$ to $8.9 \%$ failure. Batch 6252-15-0161-001 (sample 11) was the only sample having an OPyC coating that completely met the HTGR specification (Ref. 38). The OPyC failure was $1.8 \%$ for this batch. The OPyC failure of all other samples having $\sim 800 \mu \mathrm{m}$ diameters (candidate design for the FSV reactor) was $<1.8 \%$. These failures were less than the expected OHyC failure of $3 \%$ for HTGK particles (Ref. 9). The OPyC failure did not correlate with the OPyC properties of density, anisotropy, coating rate, microporosity, and active coating gas. The same results were observed for capsules HT-31 and HT-33 (Rcf. 16). The lack of correlation is not surprising, because a narrow range of OPyC properties were tested and the sample sizes were small.

Capsule HT-34 was the first capsule to test TRISO particles that had $\mathrm{H}_{2}$-diluted OPyC coatings. Samples $8,10,11$, and 13 were reference-size particles. These four samples were compared to similar particles, except the OPyC was deposited using Ar dilution, irradiated at $1200^{\circ} \mathrm{C}$ in capsules HT-31 and HT-33: The amount of OPyC failure was similar for the two diluent 
types. This indicates the similarity in irradiation performance of OPyC coatings deposited using $\mathrm{H}_{2}$ or Ar diluent.

The effect of size on the OPyC coating performance (see Section 3.1.1) was evaluated. OPyC coating failure may increase with total diameter. Sample 2 had a total diameter of $862 \mu \mathrm{m}$ and had no OPyC coating failure. The sample was irradiated to a fluence $5.1 \times 10^{25} \mathrm{n} / \mathrm{m}^{2}$ (E $>29 \mathrm{fJ}$ ) HTGR at a temperature of $1180^{\circ} \mathrm{C}$. The increase in particle diameter from 800 to $862 \mu \mathrm{m}$ did not cause OPyC coating failure at these irradiation conditions. . 


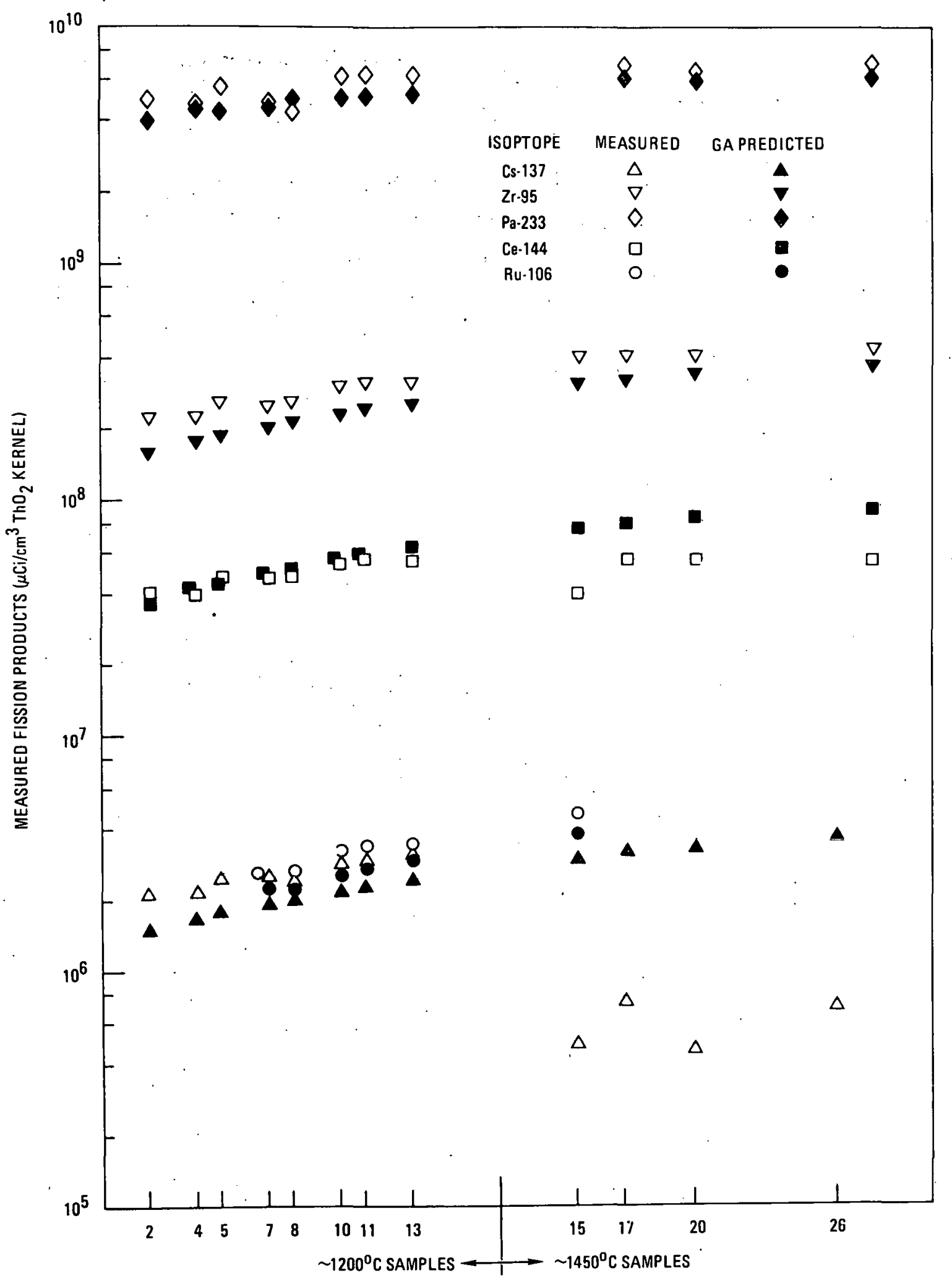

SAMPLE NUMBER (SPACED BY CAPSULE DISTANCE)'

Fig. 6-1. Measured and predicted fission products of capsule HT-34 samples 


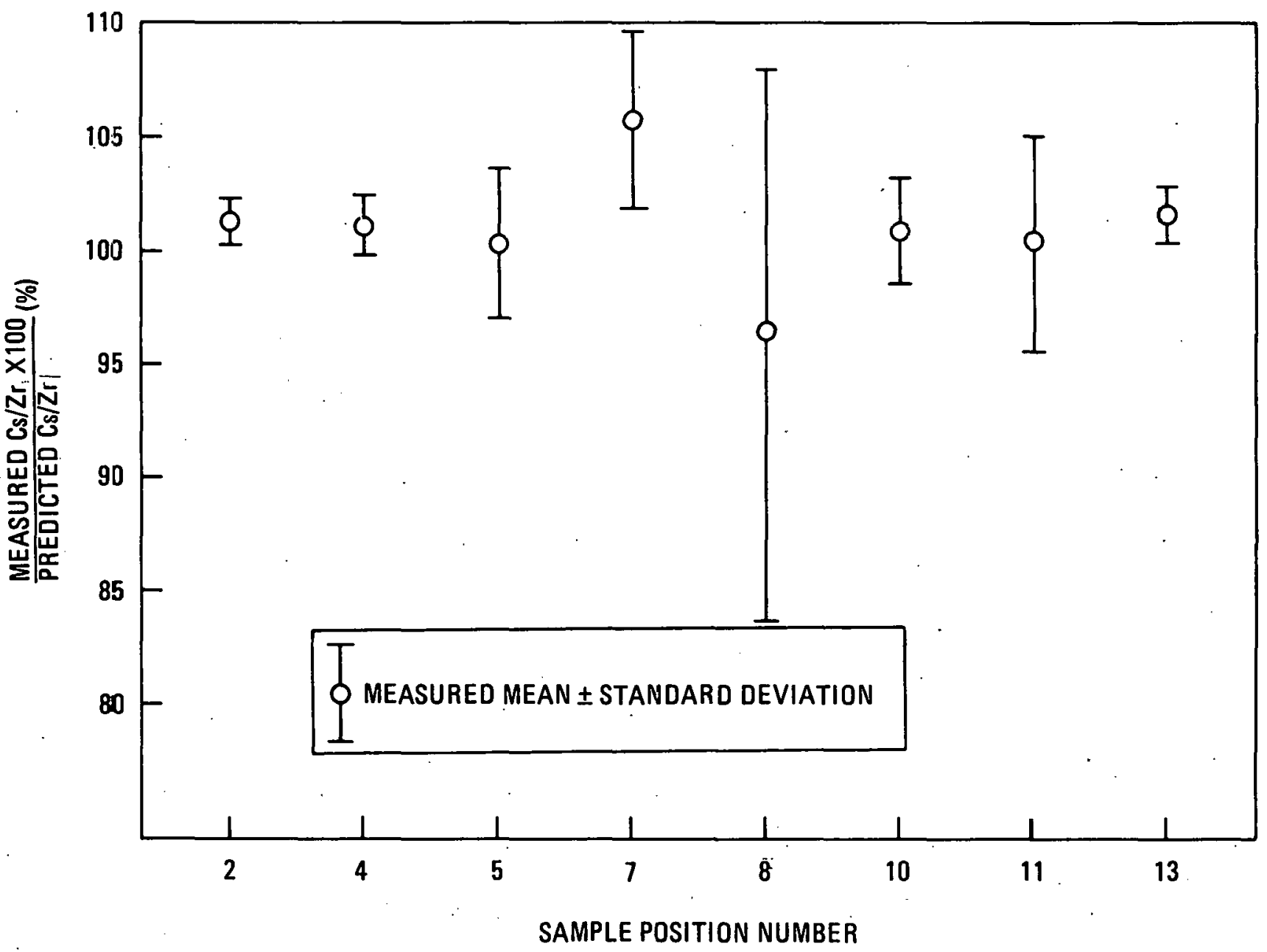

Fig. 6-2. Ratios of measured to predicted $\mathrm{C}-137 / \mathrm{Zr}-95$ ratio of $1200^{\circ} \mathrm{C}$ samples 


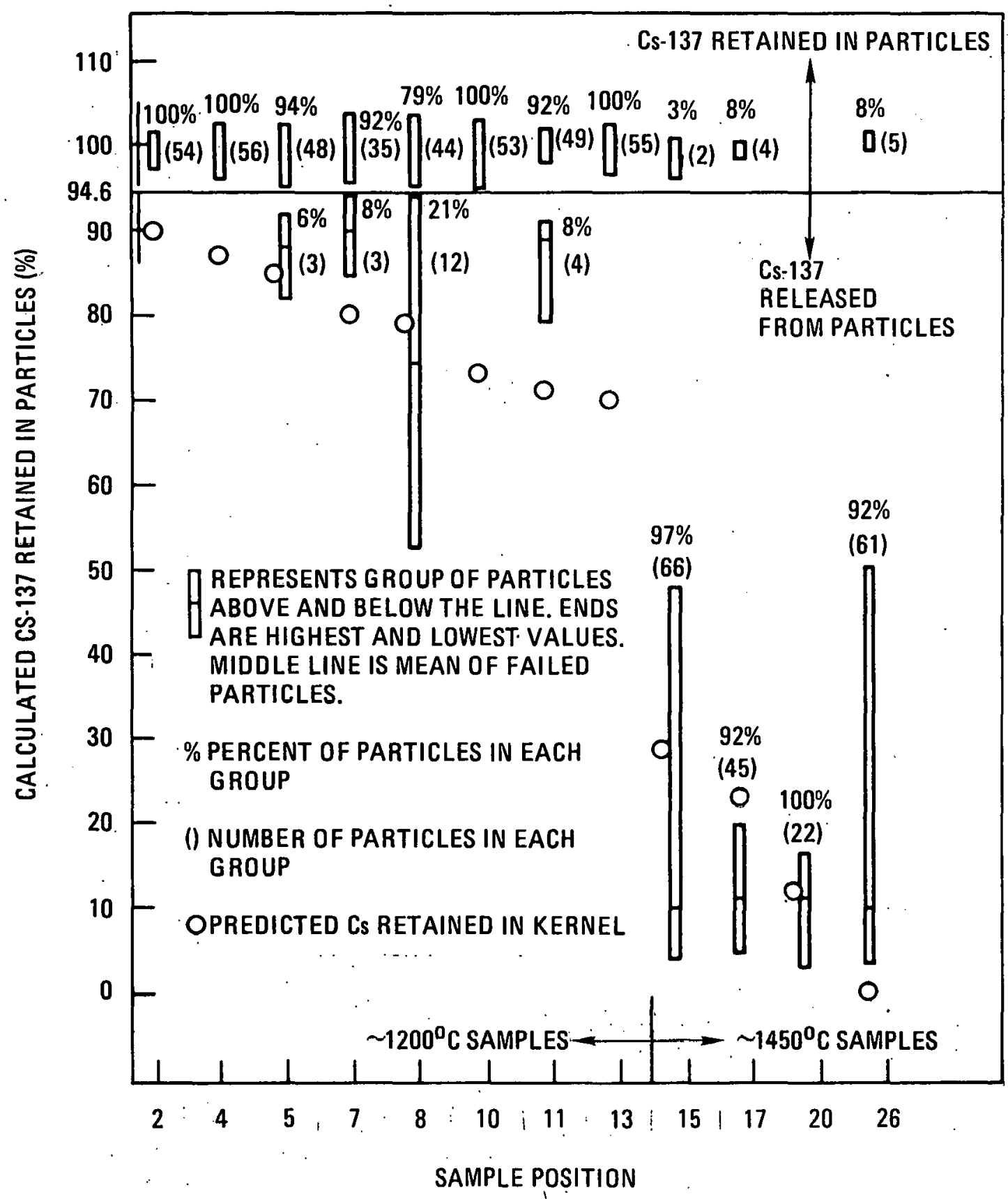

Fig. 6-3. Calculated amount of $\mathrm{Cs}-137$ retained (measured Cs/Zr/predicted Cs/Zr) in particles from each sample 


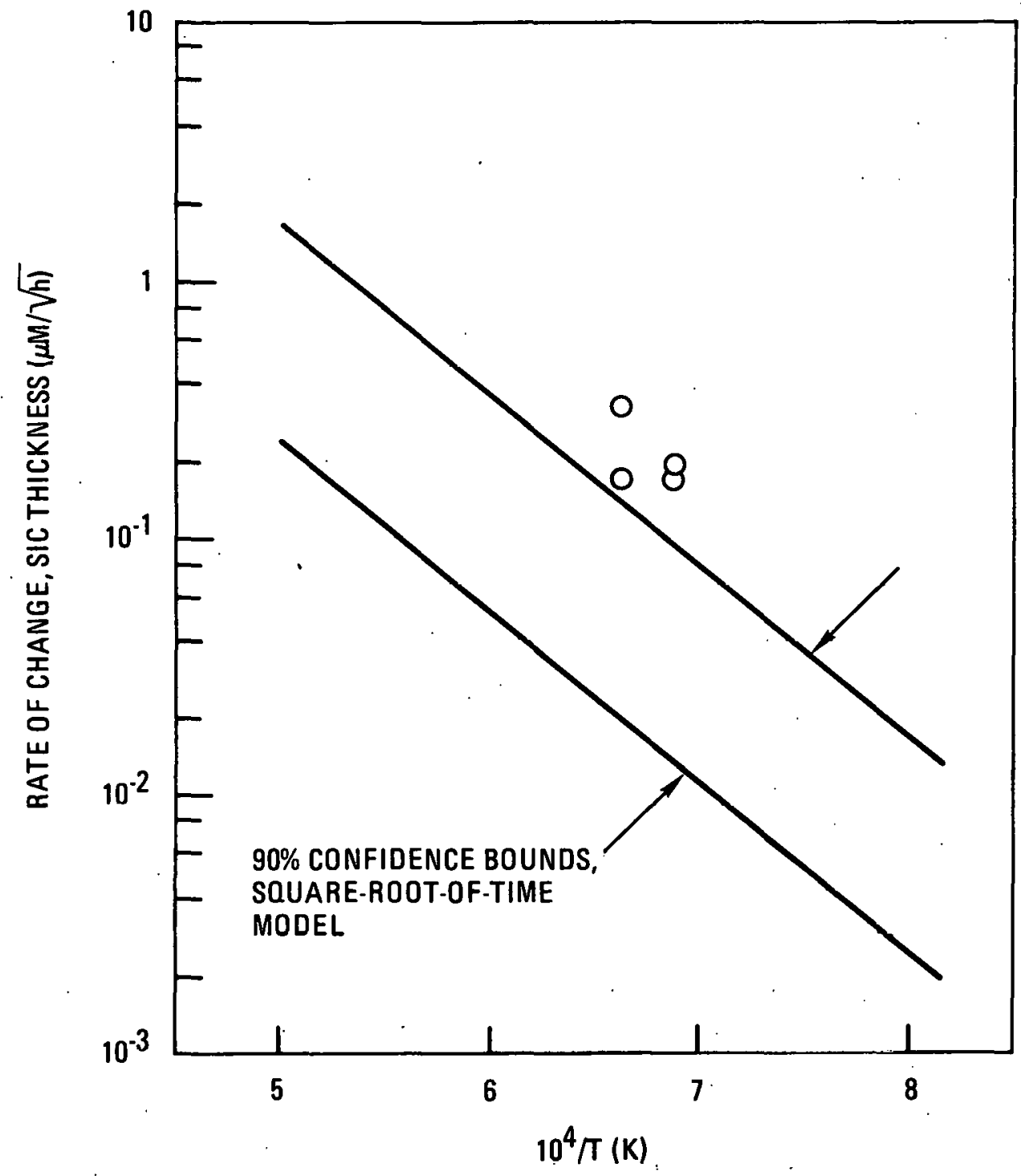

Fig. 6-4. Graph of SiC thinning versus temperature. The points given are the amount of SiC fission product attack measured on metallographic polished sections of HT- 34 TRISO ThO 2 particles. The solid lines are the bounds of the square-root-oftime model for SiC thinning (Ref. 31). 
TABLE 6-1
SLMMARY OF CDATED PARTICLE FAILURE JF TRISO ThO ${ }_{2}$ SAMPLES

\begin{tabular}{|c|c|c|c|c|c|c|c|c|c|c|c|c|c|c|c|c|}
\hline \multirow[b]{3}{*}{$\begin{array}{l}\text { Capsule } \\
\text { Position }\end{array}$} & \multirow[b]{3}{*}{$\begin{array}{c}\text { Particle 3atch } \\
\text { No. } \\
(6252-) \\
\end{array}$} & \multicolumn{6}{|c|}{ Coating vartables } & \multirow{2}{*}{\multicolumn{3}{|c|}{ Irradiation Conditions }} & \multirow[b]{3}{*}{$\begin{array}{c}\text { Predicted } \\
\text { Particle } \\
\text { Fallure } \\
(\text { (z) }\end{array}$} & \multirow{2}{*}{\multicolumn{2}{|c|}{ Visual Examination }} & \multirow{3}{*}{$\begin{array}{c}\text { Metallo- } \\
\text { graphic } \\
\text { Examination } \\
\text { Total } \\
\text { Particle } \\
\text { Fa1lure(a) }\end{array}$} & \multirow[b]{3}{*}{$\begin{array}{c}\text { Fallure } \\
\text { Based on Kr } \\
\text { Release (a,b) } \\
(z) \\
\end{array}$} & \multirow[b]{3}{*}{$\begin{array}{c}\text { Fa1lure } \\
\text { Based on } \\
\text { Release(a, }(a) \\
(z)\end{array}$} \\
\hline & & \multirow[b]{2}{*}{$\begin{array}{c}\text { Buffer } \\
\text { Thickness } \\
(\mu \mathrm{m})\end{array}$} & \multirow[b]{2}{*}{$\begin{array}{c}\text { SiC Flaw } \\
\text { Frequency } \\
\text { (x) }\end{array}$} & \multicolumn{4}{|c|}{ OPyC Coating } & & & & & & & & & \\
\hline & & & & $\begin{array}{l}\text { Deneity } \\
\text { (Mg/n } 3 \text { ) }\end{array}$ & $\mathrm{BAF}_{\mathrm{O}}$ & \begin{tabular}{|c|} 
OPyc \\
Micro- \\
porostity \\
(ml/kg)
\end{tabular} & $\begin{array}{c}\text { Coat Ing } \\
\text { Rase } \\
(\mu \mathrm{m} / \mathrm{min})\end{array}$ & \begin{tabular}{c|} 
Time- \\
Averaged \\
Temp. \\
$\left({ }^{\circ} \mathrm{C}\right)$ \\
\end{tabular} & 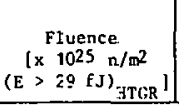 & $\begin{array}{c}\text { Burnup } \\
\text { (Z F1MA) }\end{array}$ & & $\begin{array}{c}\text { OPyC } \\
\text { Fatlureia) } \\
(z)\end{array}$ & $\begin{array}{c}\text { Total } \\
\text { Particle } \\
\text { Faflure(a) } \\
(z)\end{array}$ & & & \\
\hline \multicolumn{17}{|c|}{ Low Temperature Magazine } \\
\hline 2 & $20-0161-301$ & 83 & 6 & 1.58 & $1.0 \div 1$ & 59 & 8.4 & 1180 & 5.1 & 5.1 & 0 & $0(56)$ & $0(56)$ & ND & ND & $0(54)$ \\
\hline 4 & $07-0262-001$ & 60 & 38 & 1.80 & 1.027 & 48 & 5.0 & 1180 & 5.8 & 5.7 & 0 & $0(57)$ & $0(57)$ & .ND & $0(56)$ & $0(56)$ \\
\hline 5 & $14-0261-001$ & 27 & 29 & 1.97 & 1.040 & 54 & 7.1 & 1210 & 6.1 & 6.0 & 59 & $8.9(55)$ & $0(56)$ & $0(16)$ & $0(16)$ & $6(51)$ \\
\hline 7 & $14-0171-001$ & 25 & 9 & 1.57 & 1.041 & 57 & 7.1 & 1230 & 6.7 & 6.7 & 51 & $0(56)$ & $0(56)$ & ND & ND & $8(38)$ \\
\hline 8 & $13-0161-001$ & 57 & 16 & $1 . ; 9$ & 1.031 & 57 & 8.3 & 1220 & 7.0 & 7.0 & 0 & $0(57)$ & o (57) & ND & $1.8(56)$ & $21(56)$ \\
\hline 10 & $14-0161-001$ & 63 & 9 & 1.97 & 1.041 & 57 & 7.6 & 1240 & 7.5 & 7.6 & 0 & $1.8(57)$ & o (57) & $0(15)$ & $0(56)$ & $0(53)$ \\
\hline 11 & $15-0161-001$ & 56 & 12 & 1.81 & 1.033 & 21 & 5.0 & 1250 & 7.7 & 7.9 & 0 & $1.8(57)$ & $0(57)$ & $0(15)$ & $1.8(56)$ & $8(53)$ \\
\hline 13 & $16-0161-001$ & 57 & 12 & 1.96 & 1.048 & 25 & 5.3 & 1180 & 8.2 & 8.5 & 0 & $0(56)$ & $0(56)$ & $0(15)$ & $0(52)$ & $0(55)$ \\
\hline \multicolumn{17}{|c|}{ High Temperature Magazine } \\
\hline 15 & $20-0161-002$ & 82 & 6 & 1.98 & 1.041 & 59 & 8.0 & 1430 & 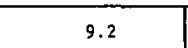 & 10.5 & 0 & $6.2(83)$ & $6.2(80)$ & $53(15)$ & ND & $97(68)$ \\
\hline 17 & $07-0262-002$ & 57 & 38 & 1.80 & 1.027 & 48 & 5.6 & 1430 & 9.4 & 10.9 & 94 & $36(81)$ & $36(81)$ & $53(15)$ & & $92(49)$ \\
\hline 18 & $14-0161-002$ & 62 & 9 & 1.97 & 1.041 & 57 & 7.6 & 1440 & 9.5 & 11.2 & 90 & & (d) - & ND & & ND \\
\hline 20 & $14-0271-001$ & 86 & 29 & 1.97 & 1.040 & 54 & 8.0 & 1440 & 9.7 & 11.6 & 11 & 71 (83) & $71(83)$ & & & $100(22)$ \\
\hline 21 & $14-0181-001$ & 91 & 10 & 1.97 & 1.041 & ' 57 & 8.1 & 1440 & 9.8 & 11.9 & 12 & $78(82)$ & $78(82)$ & & & $\mathrm{ND}$ \\
\hline 23 & $17-0161-001$ & 80 & 6 & 1.95 & 1.049 & 28 & 5.0 & 1460 & 10.0 & 12.1 & 9 & (d) & (d) & & & ND \\
\hline 24 & $15-0171-001$ & 84 & 12 & 1.31 & 1.033 & $21^{\circ}$ & 5.6 & 1450 & 10.1 & 12.4 & 4 & & (d) & & & ND \\
\hline 26 & $13-0171-001$ & 79 & 16 & 1.79 & 1.0 .31 & 57 & 8.5 & 1440 & 10.2 & 12.7 & $36^{\circ}$ & $19(85)$ & $19(85)$ & & & $92(66)$ \\
\hline
\end{tabular}

(a) Numbers in parentheses are numbers of par:1cles messured.

NOTE: ND = Not determine

(b) Measured using TR=GA reactor.

(c) Measured using ORur IMCA syscen.

(d) Could not determine due to very high coating fallure (>80x). 
TABLE 6-2

COMPARISON OF PREDICTED AND MEASURED CS RETAINED IN

FAILED TRISO $\mathrm{ThO}_{2}$ PARTICLES IN CAPSULE HT-34

\begin{tabular}{c|c|c|c|c}
\hline & $\begin{array}{c}\text { Time- } \\
\text { Averaged } \\
\text { Kerne }\end{array}$ & & \multicolumn{2}{|c}{} \\
Sample & $\begin{array}{c}\text { Semperature } \\
\text { Position }\end{array}$ & $\begin{array}{c}\text { Burnup } \\
(\% \text { FIMA) }\end{array}$ & Cs Retained (\%) \\
\cline { 4 - 5 } 5 & 1240 & 6.0 & 88 & 85 \\
7 & 1270 & 6.7 & 90 & 80 \\
8 & 1260 & 7.0 & 74 & 79 \\
11 & 1300 & 7.9 & 84 & 71 \\
15 & 1490 & 10.5 & 10 & 29 \\
17 & 1490 & 10.9 & 11 & 23 \\
20 & 1500 & 11.6 & 11 & 12 \\
26 & 1500 & 12.7 & 10 & 0 \\
\hline
\end{tabular}

(a) Time of irradiation was $9.67 \times 10^{6} \mathrm{sec}$

(b) Average of failed particles (see Fig. 6-3)

(c) Predicted $\mathrm{Cs}$ retained from $\mathrm{ThO}_{2}$ kernel 
TABLE 6-3

COMPARISON OF SIC ATTACK AND Pd PER PARTICLE OF TRISO ThO 2 PARTICLES

\begin{tabular}{|c|c|c|c|c|}
\hline \multicolumn{3}{|c|}{$\begin{array}{c}\text { Irradiation } \\
\text { Conditions (a) }\end{array}$} & \multirow{2}{*}{$\begin{array}{l}\text { Number } \\
\text { of Pd } \\
\text { Atoms per } \\
\text { Particle } \times 10^{14}\end{array}$} & \multirow{2}{*}{$\begin{array}{c}\text { Maximum } \\
\text { Pd Penetration (b) } \\
\text { in SiC } \\
(\mathrm{ym})\end{array}$} \\
\hline $\begin{array}{l}\text { Sample } \\
\text { No. }\end{array}$ & $\begin{array}{c}\text { Surface } \\
\text { Temperatures } \\
\left({ }^{\circ} \mathrm{C}\right)\end{array}$ & $\begin{array}{l}\text { Burnup } \\
(\% \text { FIMA })\end{array}$ & & \\
\hline 5 & 1210 & 6.1 & 4.6 & 0 \\
\hline 10 & 1240 & 7.5 & 5.6 & 18 \\
\hline 11 & 1250 & 7.7 & 5.9 & 9 \\
\hline 13 & 1280 & 8.2 & 6.4 & 10 \\
\hline 15 & 1430 & 9.2 & 7.8 & 0 \\
\hline 17 & 1430 & 9.4 & 8.1 & 0 \\
\hline
\end{tabular}

(a) Total irradiation time 2686 hours

(b) Measured on metallographic polished section 


\section{SUMMARY AND CONCLUSIONS}

The summary and conclusions of the evaluation of TRISO coated 450 $\mu \mathrm{m}-\mathrm{ThO}_{2}$. particles irradiated in capsule $\mathrm{HT}-34$ are the following:

1. The particle performance model over predicted the pressure-vessel failure for the two 30- $\mu \mathrm{m}$-thick-buffer samples irradiated at $1200^{\circ} \mathrm{C}$ to a burnup of $6.7 \%$ FIMA. The results showed that the model predictions were conservative. The predicted and observed pressure-yessel failure of the other $1200^{\circ} \mathrm{C}$ samples was zero.

2. The OPyC coating failure of the $\sim 800 \mu \mathrm{m}$-diameter particles (candidate design for. FSV) was $<1.8 \%$ for the samples irradiated at $\sim 1200^{\circ} \mathrm{C}$ to a fluence of 5.8 to $8.2 \times 10^{25} \mathrm{n} / \mathrm{m}^{2}$. (E $\left.>29 \mathrm{fJ}\right)_{\mathrm{HTGR}}$ and was less than the expected failure (3\%) for HTGR particles.

3. The $\mathrm{H}_{2}$-diluted OPyC coatings had irradiation performance similar to the Ar-diluted coatings at $1200^{\circ} \mathrm{C}$.

4. The variation of flaw frequency in the SiC coating did not apparently have any effect on pressure-vessel failure.

5. Two types of chemical attack of the SiC coating occurred in the $1200^{\circ} \mathrm{C}$ samples. Localized attack penetrated the SiC to a maximum of $18 \mu \mathrm{m}$ and was caused by Pd. The other type of attack was in the form of a fine porosity around the circumference of the sic. The pores were observed across the entire SiC thickness, but were concentrated on the inner surface. The corrosion may have been caused by $\mathrm{Cl}$ or. fission products. 
6. The SiC coating of the $1450^{\circ} \mathrm{C}$ samples was locally attacked at the hot side of the particle. The corrosion was most likely due to oxidation or $\mathrm{Cl}$ attack. In most particles, the attack failed the SiC coating.

7. The kernei migration was measured to be a maximum of $60 \mu \mathrm{m}$ in particles irradiated at $1430^{\circ} \mathrm{C}$ to a burnup of $10.5 \%$ FIMA. This migration distance was in good agreement with prediction.

8. In half of the $1200^{\circ} \mathrm{C}$ samples, $6 \%$ to $29 \%$, of the particles released Cs. Porosity in the SiC coating and as-coated or irradiationinduced defects in the OPyC coating apparently caused the release of Cs.

9. Almost all of the particles irradiated at $\sim 1450^{\circ} \mathrm{C}$ released $\mathrm{Cs}$, because the SiC coating had chemically corroded.

10. The observed amount of $C s$ retained by failed particles was compared to the predicted $\mathrm{Cs}$ retained in the $\mathrm{ThO}_{2}$ kernels. At $\sim 1200^{\circ} \mathrm{C}$, there was good agreement. At $1450^{\circ} \mathrm{C}$, the agrcement was fair. It was speculated that kernel migration may have caused an increasc in $\mathrm{Cs}$ release in the $1450^{\circ} \mathrm{C}$ samples. 


\section{ACKNOWLEDGMENTS}

The authors wish to acknowledge the following GA personnel:

P. R. Macy and E. T. Kuzuma for the preparation of the capsule samples and reduction of the preirradiation quality-control data; R. P. Vanek and R. 0. Whipple for manufacturing the fuel; M. D. Cronin for calculating pressure vessel model studies; M. D. Caddell for engineering calculations and data reduction of the postirradiation data; W. E. Simpson for the hot cell metallography; B. Myers for help in the fission product analysis; 0 . M. Stansfield, W. J. Scheffel, and C. L. Smith for technical advice; R. Portwood for editing this document; and the Technical Typing staff for their most efficient and expert preparation of this document.

We also express our gratitude to all the personnel at Oak Ridge National Laboratory who assisted throughout the testing of the fuel in capsule HT-34. In particular, we acknowledge the following people: K. R. Thoms for planning of the capsule operation, in-reactor operation, and operating history analysis; M. J. Kania and A. M. Howard for thermal analysis; E. L. Ryan and the staff of the High Radiation Experiment Laboratory. for capsule disassembly and visual examination; E. L. Long for his invaluable help in coordination of the entire irradiation experiment and for his technical advice; T. N. Tiegs for gamma counting the GA samples using IMGA, his patience and expertise in interpreting and evaluating the gamma scan data, and coordination of the electron microprobe examination and evaluating the microprobe results; and T. J. Hansen for the electron microprobe examination. 


\section{9.. REFERENCES}

1. Kovacs, W. J., et al., "Preirradiation Report of TRISO and BISO-Coated $\mathrm{ThO}_{2}$ Particles for Irradiation in Capsules HT-31 and HT-33," ERDA Report GA-A13923, General Atomic Company, November 1976.

2. Long, E. L., et a1., Irradiation Performance of HTGR BISO Fertile Particles in HFIR Experiments HT-17, -18, and -19," DOE Report ORNL/TM-6414, Oak Ridge National Laboratory, November 1978.

3. Scott, C. B., and D. P. Harmon, "Postirradiation Examination of Capsule F-30," ERDA Report GA-A13208, General Atomic Company, April 1975.

4. Scott, C. B., et a1., "Postirradiation Examination of Capsules P13R and P13S," ERDA Report GA-A13827, General Atomic Company, October 1976.

5. Whipple, R. O., "Batch Size Vs. Coating Properties in the 24-Cn Dry Coater-I," General, Atomic Company unpublished data, May 31, 1977.

6. "FSV Fuel Specification," General Atomic Company unpublished data.

7. Miller, C. M., "Preirradiation Characterization of FSV Fuel Test Elements FTE-1 Through FTE-8," General Atomic Company unpublished data, May 1, 1980.

8. Harmon, D. P., and C. B. Scott, "Development and Irradiation Performance of LHTGR Fuel," ERDA Report GA-A13173, General Atonic Company, October 1975.

9. Kovaks, W. J., et al., "Technical Support Document for Issue C of the HTGR Fuel Product Specification," General Atomic Company unpublished data, August 1979.

10. Kaae, J. L., et al., "Improvements in the Performance of Nuclear Fuel Particles offered by Silicon-Alloyed Carbon Coatings," Nucl. Technol., 35,536 (1977).

11. "FMB-3 Fuel Specification for Irradiation Experiments," General Atomic Company, unpublished data. 
12. "HTGR Fuels and Core Development Program. Quarterly Progress Report for the Period Ending February 28, 1978," DOE Report GA-A14863, Genera1 Atomic Company, March 1978.

13. Kaae, J. L., "Microstructures of Isotropic Pyrolytic Carbons," ERDA Report GA-A12892, General Atomic Company, March 1974.

14. Homan, F. J., and P. R. Kasten, "Gas-Cooled Reactor Programs HighTemperature Gas-Cooled Base-Technology Program. Annual Progress Report for Period Ending December 31, 1979," DOE Report ORNL-5643, Oak Ridge National Laboratory, July 1980.

15. Crockett, T. B., General Atomic Company, unpubl.ished data, September $11,1979$.

16. Sedlak, B. J., "Postirradiation Examination Report of TRISO and BISO $\mathrm{ThO}_{2}$ Particles Irradiated in Capsules HT-31 and HT-33," DOE Report GA-15544, General Atomic Company, January 1980.

17. "HTGR Base Program - Monthly Progress Report for May," Oak Ridge National Laboratory, unpublished data, June 1978.

18. Kania, M. J., "HTCAP - A Fortran IV Program for Calculating Cuating Particle Operating Temperatures in HFIR Target Irradiation Experiments," ERUA Report ORNL/TM-5332, Oak Ridge National Laboratory, May 1976.

19. Long, E. L., Oak Ridge National Laboratory, private communication, September 6, 1978 .

20. Cadde11, M. D., General Atomic Company, unpublished data, Octobcr 18, 1979.

21. McElmury, S. S., and 0. M. Stansfield, "An Analysis of the Effect of Buffer-IPyC Coating Separation on the Temperature Distribution in TRISO UC $_{2}$ Fuel Particles," ERDA Report GA-Al3500, General Atomic Company, December 1, 1975.

22. Valentine, K. H., and M. J. Kania, "IMGA Operating Manual," DOE Report ORNL/TM-6576, Oak Ridge National Laboratory, August 1979.

23. Anderson, E. E., et a1., "An In-Core Furnace for the High-Temperature Irradiation Testing of Reactor Fuels," Nucl. Techno1., 11, 259 (1971). 
24. Lofing, D. R., et al., "The Use of Gamma-Ray Spectroscopy in the Study of Fission-Product Release From Nuclear. Fuels," General Atomic Division of General Dynamics Report GA-4615, General Atomic Company, October 8, 1963.

25. "HTGR Generic Technology Program Fuels and Core Development. Quarterly Progress Report for the Period Ending August 31, 1978," ERDA Report GA-Al5093, General Atomic Company, September 1978.

26. Myers, B. F., General Atomic Company unpublished data; July 7, 1980.

27. "HTGR Fuel Development Department Technical Status Report for the Quarter Ending September 30, 1979," General Atomic Company unpublished data, October $31,1979$.

28. Homan, F., et al., "Low-Enriched Fuel Particle Performance Review," DOE Report GA-Al4759, General Atomic Company, August 1978.

29. Young, C. A., "Pre- and Postirradiation Evaluation of Fuel in Capsule HRB-14," DOE Report GA-A15969, General Atomic Company, to be published.

30. Smith, C. L., General Atomic Company unpublished dâta, September 4, 1979 .

31. "HTGR Fuel Development Department Technical Status Report for the Quarter Ending June 30, 1980," General Atomic Company unpublished data, August 1980 .

32. Brown, P. E., and R. L. Faircloth, "Metal Fission Product Behavior in High-Temperature Reactors - $\mathrm{UO}_{2}$ Coated Particle Fue1," J. Nucl. Mater., 59,29 (1976).

33. Foster, R. E., General Atomic Company unpublished data, January 24 , 1980 .

34. Stansfield, 0. M., et al., "Performance of $\mathrm{ThO}_{2}$ In HTGR Fuel Particles," DOE Report GA-A14745, General Atomic Company, March 1978.

35. Flowers, R. H., and G. W. Horsley, "The Influence of Oxide Kernels on the Manufacture and Performance of Coated Particle Fuel," Atomic Energy Research Establishment (U.K.) Report R5959, 1968. 


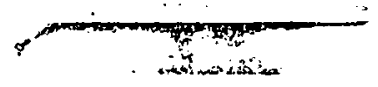

36. Homan, F. J., and E. L. Long, "Irradiation Performance of HTGR Recycle Fissile Fuel," ERDA Report ORNL/TM-5502, Oak Ridge National Laboratory, August 1976 .

37. Grubmeir, H., et al., "Silicon Carbide Corrosion in High-Temperature Gas-Cooled Reactor Fuel Particles," Nucl. Technol., 35, 413 (1977).

38. "HTGR Product Specification, Issue C," General Atomic Company unpublished data, August 1979 . 